\title{
IMPLEMENTING ION IMAGING TO PROBE CHEMICAL KINETICS AND DYNAMICS AT SURFACES
}

\author{
Dissertation \\ zur Erlangung des mathematisch-naturwissenschaftlichen Doktorgrades \\ "Doctor rerum naturalium" \\ der Georg-August-Universität Göttingen
}

im Promotionsprogramm Chemie der Georg-August University School of Science (GAUSS)

vorgelegt von

Jannis Neugebohren

aus Uelzen

Göttingen, 2018 
Betreuungsausschuss

Prof. Dr. Alec M. Wodtke

Institut für Physikalische Chemie, Georg-August-Universität Göttingen

Prof. Dr. Dirk Schwarzer

Max-Planck-Institut für Biophysikalische Chemie, Göttingen

Mitglieder der Prüfungskommission

Referent: Prof. Dr. Alec M. Wodtke

Institut für Physikalische Chemie, Georg-August-Universität Göttingen

Korreferent: Prof. Dr. Dirk Schwarzer

Max-Planck-Institut für biophysikalische Chemie, Göttingen

Weitere Mitglieder der Prüfungskommission

Prof. Dr. Jörg Behler

Institut für Physikalische Chemie, Georg-August-Universität Göttingen

Prof. Dr. Burkhard Geil

Institut für Physikalische Chemie, Georg-August-Universität Göttingen

Prof. Dr. Theofanis N. Kitsopoulos

Institut für Physikalische Chemie, Georg-August-Universität Göttingen

Prof. Dr. Konrad Koszinowski

Institut für Organische Chemie, Georg-August-Universität Göttingen

Tag der mündlichen Prüfung: 27. Juni 2018 
In this thesis I present a novel surface science apparatus and new methods to measure and analyze reaction kinetics at surfaces. By combining crossed-beam surface scattering with universal fs-laser ionization and ion imaging detection I simultaneously measure the dynamical fingerprints and the reaction rates of the products formed on the surface. As different catalytic sites might form products with distinct dynamical properties such as speed and angular distributions, this technique allows me to measure the site-selected reaction rates individually and simultaneously.

I demonstrate the potential of the method by studying $\mathrm{CO}$ oxidation by atomic oxygen on platinum surfaces. The oxidation products are known to show a bimodal distribution in angle and speed at temperatures between $500-700 \mathrm{~K}$. One component is strongly hyperthermal and shows a narrow $\cos ^{8}(\vartheta)$ distribution around the surface normal, while the other shows a cosine distribution and thermal speeds. Despite this being one of the most heavily investigated systems we are the first to show that the two different dynamical distributions actually have very different lifetimes on the surface. This contradicts the long standing assumption that the bimodality is caused by partial accommodation of the products after a single transition state.

By comparing the desorption and reaction rates of $\mathrm{CO}$ as a function of O-atom coverage and on two crystals with different step densities, $\operatorname{Pt}(111)$ with $0.25 \%$ steps and $\mathrm{Pt}(332)$ with $17 \%$ steps, I am able to identify three competing elemenary step reactions at steps and terraces.

The reaction of $\mathrm{CO}$ on terraces, $\mathrm{CO}_{\mathrm{t}}$, with $\mathrm{O}$ on terraces, $\mathrm{O}_{\mathrm{t}}$, produces hyperthermal $\mathrm{CO}_{2}$ with an activation energy of $0.6 \mathrm{eV}$ and a preexponential factor of $3.5 \times 10^{9} \mathrm{~s}^{-1}$ (defined at an O-atom coverage of $1 \mathrm{ML}$ ). This reaction channel dominates at high temperatures and high oxygen coverage. The $\mathrm{O}$-atoms on steps, $\mathrm{O}_{s}$, can either react with $\mathrm{CO}$ on steps, $\mathrm{CO}_{\mathrm{s}},\left(0.65 \mathrm{eV}, 2.9 \times 10^{9} \mathrm{~s}^{-1}\right)$ or with $\mathrm{CO}$ on terraces sites $\left(0.4 \mathrm{eV}, 5.9 \times 10^{7} \mathrm{~s}^{-1}\right)$. The reactions at steps produce thermal $\mathrm{CO}_{2}$ and dominate at low oxygen coverages and low temperatures.

Using a kinetic model with these three rate coefficients I am able to correctly predict the reaction rates as a function of temperature, step density and $\mathrm{O}$-atom coverage. This suggests that previously reported rate coefficients, which change strongly with O-atom coverage, were an erroneous result; the lack of velocity-selected kinetic information inevitably led to the wrong kinetic model. 
As the technique is widely applicable, ion imaging of processes at surfaces will soon become the method of choice for molecular beamsurface investigations.

\section{ZUSAMMENFASSUNG}

In dieser Doktorarbeit stelle ich neue Methoden zur Messung und Analyse der Reaktionskinetik an Oberflächen vor. Die Apparatur kombiniert Molekülstrahlstreuung auf Oberflächen mit universeller fs-Laser Ionisation und "ion imaging". Dies erlaubt die gleichzeitige Messung der Reaktionsgeschwindigkeit auf der Oberfläche und der dynamischen Eigenschaften der entstehenden Produkte. Verschiedene Anordnungen von Oberflächenatomen auf Katalysatoroberflächen, wie z.B. Terrassen, Stufen oder Defekte, führen zu unterschiedlicher Stabilisierung des Übergangszustands und können somit Produkte mit unterschiedlichen dynamischen Eigenschaften wie Geschwindigkeit und Winkelverteilung bilden. Die hier präsentierte Methode nutzt diese Eigenschaften und ermöglicht die reaktionsortsaufgelöste und gleichzeitige Messung verschiedener Reaktionsgeschwindigkeiten.

Das Potenzial der Methode wird anhand der Kohlenmonoxid Oxidation durch adsorbierte Sauerstoffatome an Platinoberflächen untersucht. Es ist bekannt, dass Reaktionsprodukte mit zwei sehr unterschiedlichen Winkel- und Geschwindigkeitsverteilungen gebildet werden. Ein Reaktionsprodukt verlässt die Oberfläche in einer schmalen $\cos ^{8}(\vartheta)$ Winkelverteilung mit einer hyperthermischen Geschwindigkeitsverteilung, während das andere eine breite $\cos (\vartheta)$ Winkelverteilung und eine thermische Geschwindigkeitsverteilung zeigt. Obwohl das System in zahlreichen Studien untersucht wurde, ist dies meines Wissens nach der erste experimentelle Nachweis unterschiedlicher Reaktionszeiten der Produkte auf der Oberfläche. Dies widerspricht der früher gemachten Annahme, dass die unterschiedlichen Dynamiken durch partielle thermische Equilibrierung der Produkte nach dem Übergangszustand entstehen.

Durch Vergleich von Desorptions- und Reaktionsgeschwindigkeiten von $\mathrm{CO}$ in Abhängigkeit der Sauerstoffatombedeckung und der Temperatur sowie auf zwei Pt-Kristalloberflächen mit stark unterschiedlicher Stufendichte (Pt(111), 0,25\% Stufen und Pt(332), 17\% Stufen) können drei in Wettbewerb stehende Reaktionen auf Terrassen und an Stufen identifiziert werden.

Die Oxidation von $\mathrm{CO} z u \mathrm{CO}_{2}$ auf Terrassen $\left(\mathrm{CO}_{\mathrm{t}}+\mathrm{O}_{\mathrm{t}} \rightarrow \mathrm{CO}_{2}\right)$ bildet hyperthermische Produkte mit einer Arrhenius-Aktivierungsenergie von $0.6 \mathrm{eV}$ und einem Vorfaktor von $3.5 \times 10^{9} \mathrm{~s}^{-1}$, definiert bei einer Sauerstoffatombedeckung von 1 ML. Dieser Reaktionsweg dominiert bei hohen Temperaturen und hohen Sauerstoffatombedeckungen. Sauerstoff an Stufen, $\mathrm{O}_{s}$, kann entweder mit CO an Stufen, 
$\mathrm{CO}_{\mathrm{s}},\left(0.65 \mathrm{eV}\right.$ und $\left.2.9 \times 10^{9} \mathrm{~s}^{-1}\right)$ oder mit $\mathrm{CO}_{\mathrm{t}}\left(0.4 \mathrm{eV}\right.$ und $\left.5.9 \times 10^{7} \mathrm{~s}^{-1}\right)$ reagieren. Die Reaktion an Stufen führt zu Produkten mit thermischer Geschwindigkeitsverteilung und dominiert bei niedrigen Bedeckungen und Temperaturen.

Ein kinetisches Modell mit diesen drei Elementarreaktionen kann die Reaktionsgeschwindigkeit bei verschiedenen Temperaturen, Stufendichten und Sauerstoffbedeckung korrekt vorhersagen. Vorherige Studien zu den Reaktionsgeschwindigkeiten haben stets eine starke Abhängigkeit der Aktivierungsenergie von der Sauerstoffbedeckung festgestellt. Die vorliegende Arbeit legt nahe, dass die Bedeckungsabhängigkeit der Reaktionsgeschwindigkeiten eine Folge eines fehlerhaften kinetischen Modells sind. Nur durch geschwindigkeitsaufgelöste Messung der Kinetik ist es möglich diese Fehlinterpretation aufzuklären.

Da die Kombination aus "ion imaging" und Oberflächenexperimenten einen beträchtliche Mehrwert an Informationen bietet und sehr vielseitig anwendbar ist, kann diese Methode leicht auf eine Vielzahl weiterer Systeme übertragen werden. 

Of all the things I've lost, I miss my mind the most.

— Mark Twain / Ozzy Osbourne

\section{ACKNOWLEDGMENTS}

Thank you, Alec, for giving me the opportunity to pursue a $\mathrm{PhD}$ under your supervision. I enjoyed setting up the lab, building the Beamer II apparatus and developing the new imaging methods. You managed to gather a group of great people. I especially appreciate that you encouraged critical scientific thinking early on and that everyone is allowed to speak one's mind in discussions.

Thank you, Theo, for teaching me all the tips and tricks in the lab. You have a great way of explaining things and you never miss the opportunity to tell a good joke. It was a pleasure to work with you.

I am grateful to Dan H., who accepted me as a PhD student and coworker in the lab, built the machine with me and then encouraged me to investigate an experimental problem on my own.

Thank you, Dirk, Sascha, Charlie and Dan A., for helpful and valuable discussion on the topic of this thesis. I would not have solved the CO oxidation puzzle without you - solving it was a team effort!

A special thanks goes to my fellow scientists in the lab Hinrich, Dima and Barratt. We spent many hours in the lab together and regularly discussed the experimental methods or the data acquired - I enjoyed working with you. Your help with correcting my thesis was invaluable.

I am grateful to my parents Barbara und Martin, who have always provided me through moral and emotional support in my life. And I am grateful to my girlfriend Inken, who understood that I had to work in the lab at hours and always encouraged and supported me.

And finally, to everyone in "Wodtkeland": It was a great time with all of you. I consider many of you my friends and hope to stay in close contact. Playing in the band 'Alec Wodtke and the Heartbreakers' was awesome. I would love to go on another sailing trip with you guys. Without you and the fun we had, the long hours in the lab might have gotten the best of me. I will miss the time spent with you. 



\section{CONTENTS}

I EXPERIMENTAL SETUP AND METHODS I

I INTRODUCTION 3

2 MOLECULAR BEAM-SURFACE SCATTERING APPARATUS 7

2.1 Ultra-high vacuum 8

2.2 Surface preparation and characterization 10

2.3 Molecular beam generation 12

2.4 Laser System 15

2.4.1 REMPI laser setup 15

2.4.2 Strong field ionization 15

2.4.3 Trigger setup 16

3 ION IMAGING OF GAS-PHASE REACTIONS 19

3.1 Imaging techniques 19

3.1.1 Spatial imaging 19

3.1.2 Velocity map imaging 21

3.1.3 Abel transform 22

3.1.4 Slice imaging 26

3.1.5 Event counting 27

3.2 Gas-phase imaging at the VMI machine 28

3.2.1 Experimental setup of the VMI chamber 28

3.2.2 Photoelectron spectroscopy of $\mathrm{CO}_{2} \quad 30$

3.2.3 Photofragmentation of nitrous oxide 31

3.2.4 Velocity resolution 34

$3 \cdot 3$ Conclusions 35

4 ION IMAGING OF PROCESSES AT SURFACES 39

4.1 Velocity-sensitive imaging of processes at surfaces 39

4.1.1 Challenges and limitations in previous experiments 39

4.1.2 Our experimental configuration 40

4.1.3 Ion image of $\mathrm{N}_{2}$ scattering - an example

4.2 Dynamical parameters from ion images 43

4.2.1 Coordinate system 44

4.2.2 Angular distributions from ion images 45

4.2.3 Density to flux in imaging 48

4.2.4 Speed distributions in surface scattering experiments 49

4.2.5 Speed distributions from ion images 52

4.3 Image processing 53

4.3.1 ROI vs delay 53

4.3.2 Background signal correction 53

4.3.3 Kinetic rates versus speed 55

4.4 Velocity-map imaging of processes at surfaces $\quad 56$ 
II CO OXIDATION ON PLATINUM SURFACES 59

5 CO TRAPPING-DESORPTION ON PT 6I

5.1 Trapping-desorption on surfaces 61

5.2 Trapping-desorption of CO on $\mathrm{Pt}(111) \quad 62$

5.2.1 Kinetic equations 64

5.2.2 Convolution with the incoming beam 64

5.2.3 Results - CO on Pt(111) 65

$5 \cdot 3$ Trapping-desorption of $\mathrm{CO}$ on $\mathrm{Pt}(332) \quad 67$

5.3.1 Results: CO on $\mathrm{Pt}(332) \quad 68$

5.4 Discussion 70

6 CO OXIDATION ON PT - INTRODUCTION 73

6.1 Experimental setup 73

6.2 Ion images of $\mathrm{CO}_{2}$ at different reaction conditions 74

6.3 Kinetic traces from ion images $\quad 78$

6.4 Kinetic model from experimental observations $\quad 87$

7 CO OXIDATION ON PT - RESULTS 95

7.1 Kinetic model 95

7.1.1 Reaction scheme 95

7.1.2 Differential equations 96

7.1.3 Numeric approximation to differential equations

7.1.4 Perturbation of the equilibrium by incoming beams

7.1.5 Adsorption from incoming beams 99

7.2 Oxygen titration experiment 100

7.2.1 Calibration on a fully covered surface 100

7.2.2 From titrations to total $\mathrm{CO}_{2}$ yield IOI

7.2.3 $\mathrm{CO}_{2}$ flux vs titration time 104

7.2.4 Titration of a partially covered surface 106

7.2.5 Result: O-atom coverage vs RRR 106

7.3 Distribution of adsorbates between terrace and step sites 108

7.3.1 Equilibrium parameters from the canonical partition function $\quad 108$

7.3.2 Titration combined with partition function 112

7.4 Result of the Kinetic Model 114

7.4.1 Input values for the numeric solution $\quad$ II4

7.4.2 Python code structure 117

7.4.3 Fit result on $\operatorname{Pt}(111) \quad 118$

7.4.4 Fit result on $\mathrm{Pt}(332) \quad 120$

$7 \cdot 5$ Self-consistency checks 121

7.5.1 Sensitivity analysis 121

7.5.2 Steady-state O-atom concentrations 124

8 CO OXIDATION ON PT - DISCUSSION 129

8.1 Discussion of the results 129

8.1.1 Angular distribution 129

8.1.2 Speed and kinetic energy distribution 131

8.1.3 CO oxidation mechanism on platinum $\quad 135$ 
8.1.4 Effective conversion rate 138

8.1.5 Activation energies 141

8.2 Modeling approach 143

8.3 Review: Imaging of reactions at surfaces 145

8.4 Impact of the results 146

III BIBLIOGRAPHY 157

BIBLIOGRAPHY 159

IV APPENDIX 171

A APPENDIX: KINETIC MODEL RESUlT PLOTS 173

A.1 $\operatorname{Pt}(111)$ results 173

A.2 $\mathrm{Pt}(332)$ results 188

B APPENDix: Python CODE Utilities 197

B.1 Speed distributions in the Beamer II analysis code 197

B.2 How to fit Arrhenius parameters separately 198

B.3 Python class structure of the kinetic model 200

C EXTENDED EXPERIMENTAL TECHNIQUES AND TIPS 203

C.1 Conversion of pixels to $\mathrm{mm} 203$

C.2 Multiple beam pulse timing 204 
Figure 2.1

Figure 2.2

Figure 2.3

Figure 2.4

Figure 2.5

Figure 2.6

Figure 2.7

Figure 2.8

Figure 2.9

Figure 2.10

Figure 3.1

Figure 3.2

Figure 3.3

Figure 3.4

Figure 3.5

Figure 3.6

Figure 3.7

Figure 3.8

Figure 3.9

Figure 3.10

Figure 3.11

Figure 3.12

Figure 3.13

Figure 3.14

Figure 3.15

Figure 3.16

Figure 4.1

Figure 4.2

Figure $4 \cdot 3$

Figure 4.4

Figure 4.5

Figure 4.6

Figure 4.7

Figure 4.8

Figure 4.9

Figure 4.10

Figure 4.11

Figure 4.12
Beamer II schematic $\quad 7$

Beamer II pumps and pressures

$\mathrm{Au}(111)$ surface structure 10

$\mathrm{Au}(111)$ Auger $\quad 11$

Platinum Auger $\quad 11$

Pt(111) AFM 12

platinum surface structure 13

Home-built PZT valve $\quad 14$

Trigger pulses ns-laser $\quad 16$

Trigger setup fs-laser $\quad 17$

Spatial imaging sequence 19

Spatial imaging setup of Chandler and Hous-

ton 20

VMI setup of Eppink and Parker 21

3D Abel transform 23

Projection in ion images 24

Polar coordinate system 24

Slice imaging technique 25

Slice imaging technique $\quad 27$

Ion spot size 28

Side view of the VMI chamber $\quad 28$

VMI source chamber 29

$\mathrm{CO}_{2}$ PES image 30

Schematic illustration of the $\mathrm{N}_{2} \mathrm{O}$ photodissociation 31

Ion image of $\mathrm{N}_{2} \mathrm{O}$ fragments 32

Vibrational population of $\mathrm{N}_{2} \mathrm{O} \quad 33$

Demonstration of VMI resolution using $\mathrm{N}_{2}$ imag-

ing 34

First imaging of processes at surfaces setup $\quad 39$

Imaging setup of Kobrin et al. $\quad 40$

Imaging unit arrangement 41

Ion image of $\mathrm{N}_{2}$ scattered from $\mathrm{Au}(111) \quad 43$

Imaging geometry of processes at surfaces $\quad 44$

Detector geometry in angular distributions $\quad 45$

Angle-integrated ion image $\quad 47$

Angular distribution $\quad 47$

Observed velocities 49

$\mathrm{D}_{2} \mathrm{~F}$ conversion array 50

Comparison of integration regions 52

Correction of background signal in kinetic scans $\quad 54$ 
Figure 4.13 Multiple box approach 55

Figure 5.1

Ion image of $\mathrm{CO} \quad 63$

Figure 5.2

Flux of desorbing $\mathrm{CO} \quad 63$

Figure 5.3

Figure 5.4

Figure 5.5

Figure 5.6

Figure 6.1

Figure 6.2

Figure 6.3

Figure 6.4

Figure 6.5

Figure 6.6

Figure 6.7

Figure 6.8

Figure 6.9

Figure 6.10

Figure 6.11

Figure 6.12

Figure 6.13

Figure 6.14

Figure 7.1

Figure 7.2

Figure $7 \cdot 3$

Figure $7 \cdot 4$

Figure $7 \cdot 5$

Figure 7.6

Figure 7.7

Figure 7.8

Figure 7.9

Figure 7.10

Figure 7.11

Figure 7.12

Figure 7.13

Figure 7.14

Figure 7.15

Figure 7.16

Figure 8.1

Figure 8.2

Figure 8.3

Figure 8.4

Figure 8.5

Figure 8.6

Fit to the CO/Pt(111) desorption flux 66

Arrhenius plot of CO lifetime on $\mathrm{Pt} \quad 67$

Fit to the $\mathrm{CO} / \mathrm{Pt}(332)$ desorption flux 69

Overview: Desorption rates 71

Ion image of $\mathrm{CO}_{2} \quad 75$

Ion images vs reaction conditions $\quad 77$

Kinetic profile extraction $\quad 78$

Kinetic profile: density vs flux $\quad 79$

$\mathrm{CO}_{2}$ speed distribution 80

Angular distribution of $\mathrm{CO}_{2} \quad 81$

Scaled kinetic profile $\quad 82$

TOF correction schematic 83

Final kinetic trace $\quad 84$

Kinetic traces vs RRR and T 85

Contribution to total flux 86

Single-exp. fit to kinetic trace $\quad 89$

Empirical reaction scheme $\quad 89$

Assigned reaction scheme 91

$3 \mathrm{D}$-comparison kinetic trace 102

$\mathrm{CO}_{2}$ flux estimate in titrations 103

Titrations at $653 \mathrm{~K} \quad 105$

Titration result on $\mathrm{Pt}(111) \quad 107$

Titration result on $\mathrm{Pt}(332) \quad 108$

Occupancies vs $\left[\mathrm{O}_{\mathrm{a}}\right] \quad 110$

Step-terrace preferences vs T 111

Equilibrium constant vs T III

Equilibrium coverages vs RRR on Pt(111) 112

Equilibrium coverages vs RRR on Pt(332) $\quad 113$

Numeric solution output 115

Simplified Python flow chart 117

$\mathrm{Pt}(111)$ result at $593 \mathrm{~K} \quad 119$

Sensitivity of the activation energies 123

Kinetic model output: $\mathrm{Pt}(111)$ O-atom concentrations 124

Kinetic model output: $\mathrm{Pt}(332) \mathrm{O}$-atom concentrations $\quad 126$

Angular distribution of $\mathrm{CO}_{2} \quad 130$

Comparison of flux velocity distribution to literature $\quad 132$

Minimum energy path calculation 134

$\mathrm{Pt}$ (111) row distance 139

Comparison of the tt-reaction to Wintterlin $\quad 143$

Heat map: CO oxidation efficiency 146 
Figure 8.7 Heat map: Hyperthermal fraction 147

Figure 8.8 Comparison of TST and exp. values $\quad 150$

Figure A.1 Full kinetic traces on $\mathrm{Pt}(111) \quad 175$

Figure A.2 Full kinetic traces on $\mathrm{Pt}(332) \quad 189$

Figure B.1 Arrhenius parameter interaction 198

Figure B.2 Python code structure 201

Figure C.1 Screen size calibration image 203

Figure C.2 Multiple beam pulse timing 204

\title{
LIST OF TABLES
}

Table 4.1

Table 5.1

Table $7 \cdot 1$

Table 7.2

Table $7 \cdot 3$

Table 8.1

Table 8.2

Table 8.3

Table 8.4
Characteristic speeds $\quad 5^{1}$

Arrhenius parameters 70

Kinetic model input values 116

Fit result on $\mathrm{Pt}(111)$ - rate coefficients 120

Fit result on $\mathrm{Pt}(332)$ - rate coefficients 121

Kinetic energy distribution 133

Result: rate coefficients 138

Diffusion rate coefficients for $\mathrm{CO}$ on $\mathrm{Pt}(111) \quad 140$

Comparison of diffusion speed to kinetic model results 140

\section{ACRONYMS}

\author{
AES Auger electron spectroscopy \\ AFM atomic force microscopy \\ DFT density-functional theory \\ LH Langmuir-Hinshelwood \\ FWHM full width at half maximum \\ MB Maxwell-Boltzmann \\ ML monolayer \\ MPI multiphoton ionization \\ MCP multi channel plate \\ ODE ordinary differential equation
}


PES potential energy surface

PZT lead zirconate titanate $\left(\mathrm{Pb}\left[\mathrm{Zr}_{\chi} \mathrm{Ti}_{1-x}\right] \mathrm{O}_{3}\right)$

QMS quadrupole mass spectrometer

REMPI resonance-enhanced multi photon ionization

RGA residual gas analyzer

ROI region of interest

RRR repetition rate ratio

SFM sum frequency mixing

SHG second harmonic generation

SNR signal-to-noise ratio

STM scanning tunneling microscope

THG third harmonic generation

TMP turbo-molecular pump

TOF time-of-flight

TPD temperature programmed desorption

TPRS temperature programmed reaction spectroscopy

UHV ultra-high vacuum

UVFS ultraviolet-grade fused silica

VMI velocity-map imaging 



\section{Part I}

\section{EXPERIMENTAL SETUP AND METHODS}

After a general introduction to the field in chapter $I$ and the experimental setup in chapter 2, I illustrate typical techniques used in gas-phase imaging in chapter 3 and show examples of measurements made at our VMI machine. In chapter 4, I describe the implementation of ion imaging in a surface scattering experiment. I transfer the imaging techniques to cover the new geometric situation and show the analysis methods used in this work.

This part of the thesis is aimed at both researchers implementing the ion imaging technique in their experiments or trying to reproduce our results, and at students new to the apparatus. The explanations in the imaging method section are quite thorough - a reader familiar with imaging and interested in the results for carbon monoxide on platinum might instead skip to chapter 5 on page 61 . 

Chemical reactions at surfaces are of paramount importance to chemical and energy industries. Most large-scale chemical processes rely on heterogeneous catalysis, where reactants and catalysts are separated in different phases and the reaction takes place at the phase boundary. The most important industrial application today is the Haber-Bosch process, which produces ammonia from its elements and accounts for about $1 \%$ of the global energy consumption [1]. The mass production of ammonia-based nitrogen fertilizers led to a significant increase in crop yields allowing modern agriculture to supply an ever larger population; nitrogen fertilizers are responsible for feeding about $50 \%$ of the world population [2].

The difficulty of the process developed in 1906-1911 by Fritz Haber (Nobel prize 1918) is that thermodynamic equilibrium is on the reactant side at high temperatures while the reaction is too slow at low temperatures. In order to allow conversion at low temperatures a suitable catalyst is required, which led to an exhaustive screening with over 2500 different iron oxide based catalyst candidates by Alwin Mittasch [3]. Lastly, the equilibrium is shifted to the product side at higher pressures which was exploited in a high pressure reactor by Carl Bosch (Nobel prize in 1931).

The mechanism of ammonia synthesis was discovered around 1990 by Gerhard Ertl (Nobel prize 2007), when modern tools in surface science became available. As a catalyst screening can be quite costly, a desirable approach would be to predict the catalytic activity based on $a b$ initio quantum chemical methods. As Ertl states, an optimal catalyst cannot be designed unless the underlying elementary steps are well understood [4]. A broad range of experiments have been conducted since then, leading to significantly increased theoretical understanding.

As computing power continues to increase exponentially, this holy grail of predicting reactivity seems to be within our grasp. Chemical kinetics and dynamics at surfaces can now be simulated with more detail and accuracy than ever, and thus require top-notch experimental results for comparison. The goal of researchers should thus be to design experiments which can be easily compared to theoretical calculations. This requires clear assignment of the observed processes, ideally elementary step processes, and the related rate coefficients and dynamical information. In order to correctly assign the observed processes the experiment must thus be carried out for the simplest possible system. As inorganic catalysts possess different sites with 
distinct activity, such as closed-packed terraces, step edges or defect sites, reactions often take place at different sites. As the discrimination between the different pathways is extraordinarily difficult, most experiments measure a combination of reactions taking place at different active sites. Unless the reaction at other sites can be suppressed, this limits mechanistic understanding and makes meaningful comparison to theory impossible.

A recent example of a well-defined experiment leading to improved theoretical understanding is the study of hydrogen atom scattering of metal surfaces by Bünermann et al. [5] and the theoretical description based on density functional theory by Janke et al. [6]. The limited range of experiments accessible to the apparatus used by Bünermann et al. motivated the development of a universally applicable experimental technique.

In this thesis I present the Beamer II apparatus we constructed during my time as a PhD student. By implementing ion imaging detection in a state-of-the-art two-molecular-beam-surface experiment we can measure chemical kinetics and dynamics at surfaces in unprecedented detail. The ion imaging detection allows the simultaneous and resolved detection of several velocity components. For systems where reaction products from different sites exhibit a specific dynamical fingerprint in the velocity distribution, this allows us to measure active-site selected kinetics at surfaces - I demonstrate this potential by investigating the oxidation of carbon monoxide on platinum surfaces. By measuring the velocity-resolved product flux as a function of molecular beam-laser delay we determine the exact time at which the reaction product desorbs from the surface. In combination with knowledge of the incident molecular beam arrival time on the surface we calculate the product flux as a function of reaction time on the surface, the kinetic trace. The comprehensive information we obtain from kinetic traces at a range of different surface conditions and two different platinum surfaces allows us to discard the previously assumed reaction mechanism and to report a more elaborate kinetic model explaining all experimental evidence seen so far.

\section{REFERENCES}

[1] Barry E. Smith. "Nitrogenase Reveals Its Inner Secrets." In: Science 297.5587 (Sept. 2002), pp. 1654-1655. DOI: $10.1126 /$ science. 1076659 (cit. on p. 3).

[2] Jan Willem Erisman, Mark A. Sutton, James Galloway, Zbigniew Klimont, and Wilfried Winiwarter. "How a century of ammonia synthesis changed the world." In: Nature Geoscience 1.10 (2008), pp. 636-639. DOI: 10.1038/ngeo325 (cit. on p. 3).

[3] Alwin Mittasch. Geschichte der Ammoniaksynthese. Weinheim: Verlag Chemie, 1951 (cit. on p. 3). 
[4] Gerhard Ertl. "Elementarschritte bei der heterogenen Katalyse." In: Angewandte Chemie 102.11 (Nov. 1990), pp. 1258-1266. DOI: 10.1002 /ange. 19901021108 (cit. on p. 3).

[5] Oliver Bünermann, Hongyan Jiang, Yvonne Dorenkamp, Alexander Kandratsenka, Svenja M. Janke, Daniel J. Auerbach, and Alec M. Wodtke. "Electron-hole pair excitation determines the mechanism of hydrogen atom adsorption." In: Science 350.6266 (2015), pp. 1346-1349. Dor: 10.1126/science. aad4972 (cit. on p. 4).

[6] Svenja M. Janke, Daniel J. Auerbach, Alec M. Wodtke, and Alexander Kandratsenka. “An accurate full-dimensional potential energy surface for $\mathrm{H}-\mathrm{Au}(111)$ : Importance of nonadiabatic electronic excitation in energy transfer and adsorption." In: Journal of Chemical Physics 143.12 (2015). DoI: 10 . 1063/1. 4931669 (cit. on p. 4). 

MOLECULAR BEAM-SURFACE SCATTERING APPARATUS

The Beamer II apparatus comprises three different sections:

- The source chamber, which houses two supersonic beam valves and two differential pumping stages.

- The main chamber, which contains the ion optics, the multi channel plate (MCP)/Phosphor screen detector, windows for laser access and the residual gas analyzer (RGA).

- The preparation chamber, which includes a leak valve/ion gun, an Auger spectrometer and the sample manipulator to move the sample between the main and the preparation chamber. The preparation chamber can be separated from the main chamber by closing the interjacent slide valve.

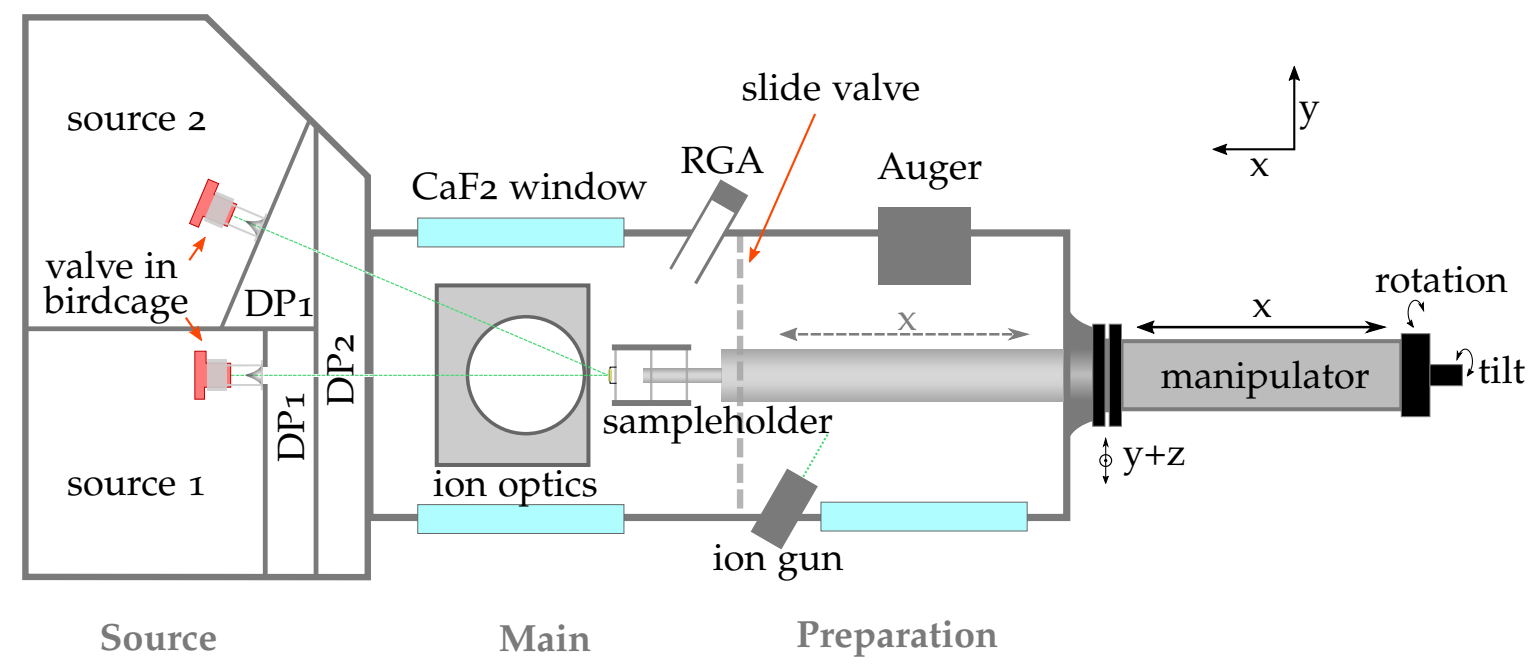

Figure 2.1: Top-down schematic of the Beamer II apparatus.

A schematic of the layout is shown in figure 2.I. The two molecular beams from the source region (left) each pass through two differential pumping stages (DP1 and $\mathrm{DP}_{2}$ ) and intersect right behind the ion optics in the main chamber. The sample, located at the tip of the manipulator can be moved to the point of intersection. Prior to surface experiments, the sample can be cleaned using the ion gun (STAIB instruments, IG-5-C) in the preparation chamber and analyzed using Auger electron spectroscopy (Staib instruments, ESA 100). Background pressures during the experiment can be monitored with an RGA (SRS, RGA 200). 


\subsection{ULTRA-HIGH VACUUM}

An important requirement for gas-surface experiments is keeping the surface free from contaminations. The results of a reaction at a surface might be strongly biased if the surface is poisoned by adsorption of undesired residual gas molecules. Controlling the background gas load in the chamber is an important means of reducing this interference. The definition of a Langmuir is a $1 \mathrm{~s}$ exposure at a pressure of $1.3 \times 10^{-6} \mathrm{mbar}$, which corresponds to approximately 1 monolayer (ML) exposure. We can thus estimate the time it takes to form a monolayer to be several hours at $1 \times 10^{-10} \mathrm{mbar}$. Establishing ultra-high vacuum (UHV) conditions, i.e. a base pressures on the order $10^{-9}-10^{-10} \mathrm{mbar}$, was therefore an essential requirement when constructing the Beamer II apparatus.

In order to achieve this, several conditions had to be fullfilled. First, great care was taken to use UHV-compatible materials.

- The machine was made out of stainless steel, which was annealed prior to construction to outgas $\mathrm{H}_{2}$ from the bulk material.

- Metal-fitted ultraviolet-grade fused silica (UVFS) windows were used where possible.

- Differentially pumped $\mathrm{CaF}_{2}$ windows were used for laser access.

- The sample holder was made out of stainless steel/tantalum. Any cable insulation was made from UHV-compatible polymers, i.e. Kapton or a similar material.

- The nickel grids for the ion optics were glued with graphite.

- Non-conducting spacers on the ion optics and the sample holder were made of either polyether ether ketone (PEEK) or Macor, a machineable glass-ceramic.

- Modifications or replacements were cleaned in an ultrasonic bath using a watery solution of Tickopur RW 77 (an ammonia cleaning agent) and methanol to remove any oil traces.

Second, a large number of turbo-molecular pumps (TMPs) are used to evacuate the various chambers, as shown in figure 2.2. All TMPs are backed by dry scroll pumps to obtain foreline pressures of $10^{-3}$ $10^{-4}$ mbar. Scroll pumps were chosen over rotary vane pumps to maintain an oil-free environment. Great care must be taken when working with oil pumps to avoid leakage of oil vapors into the UHV chamber. Hydrocarbons from oil vapor are one of the main contaminations in many UHV chambers and very difficult to remove once inside the chamber. 


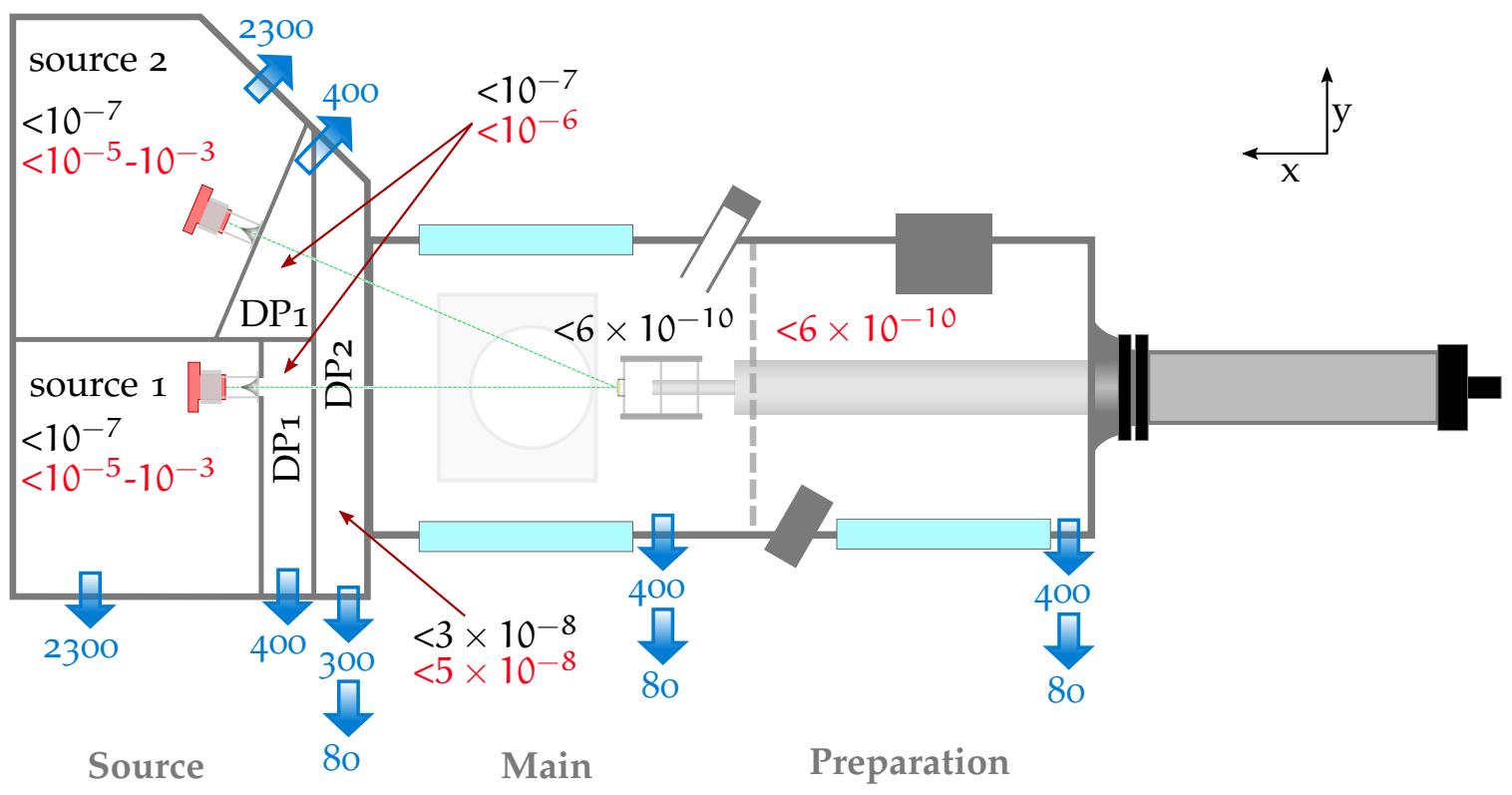

Figure 2.2: Beamer II pump schematic: The blue arrows indicate TMPs and their respective pumping speeds in $\mathrm{Ls}^{-1}$ are annotated in blue. The resulting pressure in mbar in each chamber without load is annotated in black and the pressure under load is given in red.

Each source chamber is evacuated by its own TMP (Adixen, ATP $2300 \mathrm{M}$, corrosive resistant) as are the first differential pumping stages (Pfeiffer HiPace $700 \mathrm{M}$ ). The second differential pumping stage (Pfeiffer HiPace $300 \mathrm{M}$ ) is shared by the two molecular beams. The main and preparation chambers are each evacuated by their own TMP (Pfeiffer HiPace $700 \mathrm{M}$ ). As can be estimated by the annotated values in figure 2.2 the pressure under load decreases by about two orders of magnitude per additional pumping stage.

The outlet of the TMPs at DP2, the main chamber, and the preparation chamber are further evacuated down to $10^{-6} \mathrm{mbar}$ by small $80 \mathrm{~L} \mathrm{~s}^{-1}$ booster-TMPs (Pfeiffer HiPace $8 \mathrm{oM}$ ), which are themselves backed by scroll pumps. Altogether, proper evacuation of the apparatus uses a total of 10 TMPs and 6 scroll pumps (Agilent TriScroll, 300-60o $\mathrm{L} \mathrm{min}^{-1}$ and Edwards $\left.\mathrm{n} \mathrm{X}^{\mathrm{ds}} 10 \mathrm{i}, 167 \mathrm{~L} \mathrm{~min}^{-1}\right)$. The resulting base pressures without the molecular beams running are annotated in black and the pressures under load are shown in red.

Lastly, after each time the machine is opened, it needs to be "baked" for 3 days at $90-110^{\circ} \mathrm{C}$. The increased temperature helps to desorb contaminants while pumping down. No higher temperatures than $90{ }^{\circ} \mathrm{C}$ should be used around the ion optics in order to preserve the flatness of the nickel grids. 


\subsection{SURFACE PREPARATION AND CHARACTERIZATION}

The preparation of metal single crystals prior to experiments followed standard procedures as reported by Musket et al. [7]. The surfaces used in this work and the respective handling is briefly described below.

\section{$A u(111)$}

The $\mathrm{Au}(111)$ surface is a special cut of the face-centered cubic (fcc) gold crystal. The Miller index [8] (h k l) = (111) describes the vector normal to the surface plane, where $h, k$ and 1 are the multiples along the three axes of the fcc unit cell. The fcc cell and the three components of the (111) vector are shown in panel (a) of figure 2.3. Six atoms of the fcc unit cell lie the (111) plane (magenta area). The

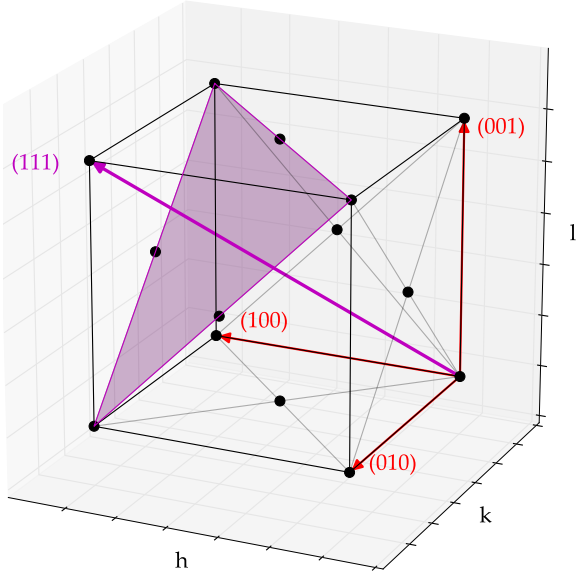

(a) FCC cell

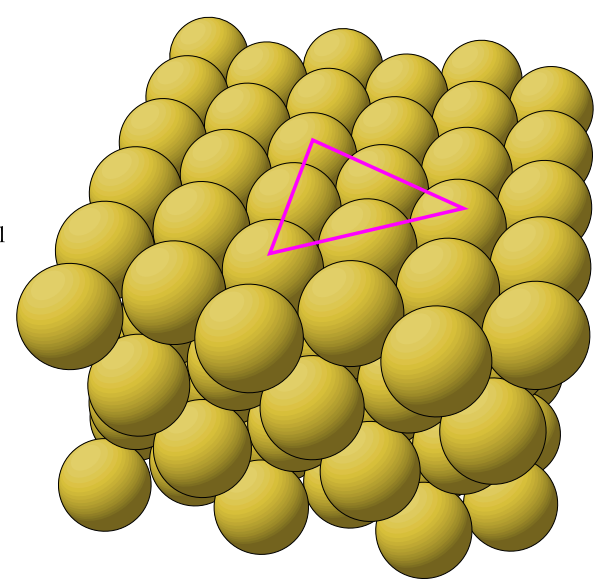

(b) $\mathrm{Au}(111)$ surface

Figure 2.3: $\mathrm{Au}(111)$ surface structure.

resulting structure is indicated by the magenta triangle in panel (b). Compared to other cuts through the fcc crystal the (111) surface is especially close-packed.

The $\mathrm{Au}(111)$ crystal was sputtered for $30 \mathrm{~min}$ with $6 \mu \mathrm{A}$ of $3 \mathrm{keV}$ argon ions and afterwards annealed for $30 \mathrm{~min}$ at $970 \mathrm{~K}$ before each experiment. An Auger spectrum was recorded to check for impurities. An introduction to the Auger-Meitner-effect [9, 10] can be found in Michael Schwarzer's bachelor thesis [11]. A typical spectrum is shown in figure 2.4. The main impurities present in gold crystals according to Musket et al. are carbon $(272 \mathrm{eV})$, sulfur $(152 \mathrm{eV})$ and calcium $(291 \mathrm{eV})$. None of these Auger peaks can be seen in the recorded spectrum. 


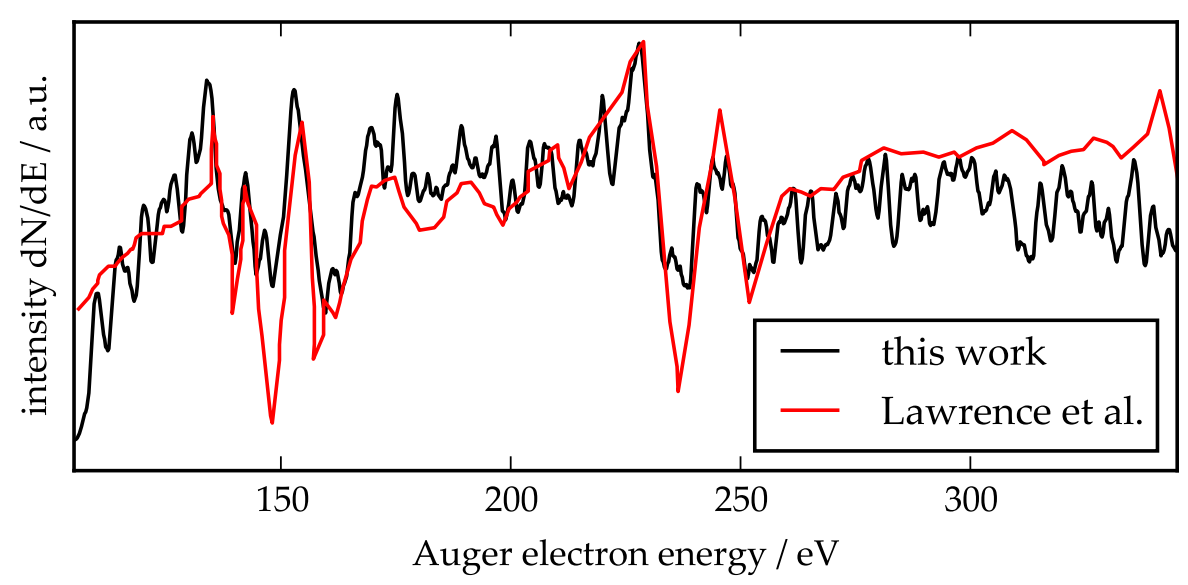

Figure 2.4: $\mathrm{Au}(111)$ Auger spectrum at $3 \mathrm{keV}$ : The black curve is an Auger spectrum we recorded after smoothing. The red curve is a digitized spectrum from the Handbook of Auger Electron Spectroscopy [12].

Pt(111) and Pt(332)

Both platinum crystals were sputtered for 30 min with $6 \mu \mathrm{A}$ of $3 \mathrm{keV}$ argon ions and afterwards annealed for $30 \mathrm{~min}$ at $1300 \mathrm{~K}$ before each experiment. An Auger spectrum was recorded to check for impurities. An example is shown in figure 2.5 .

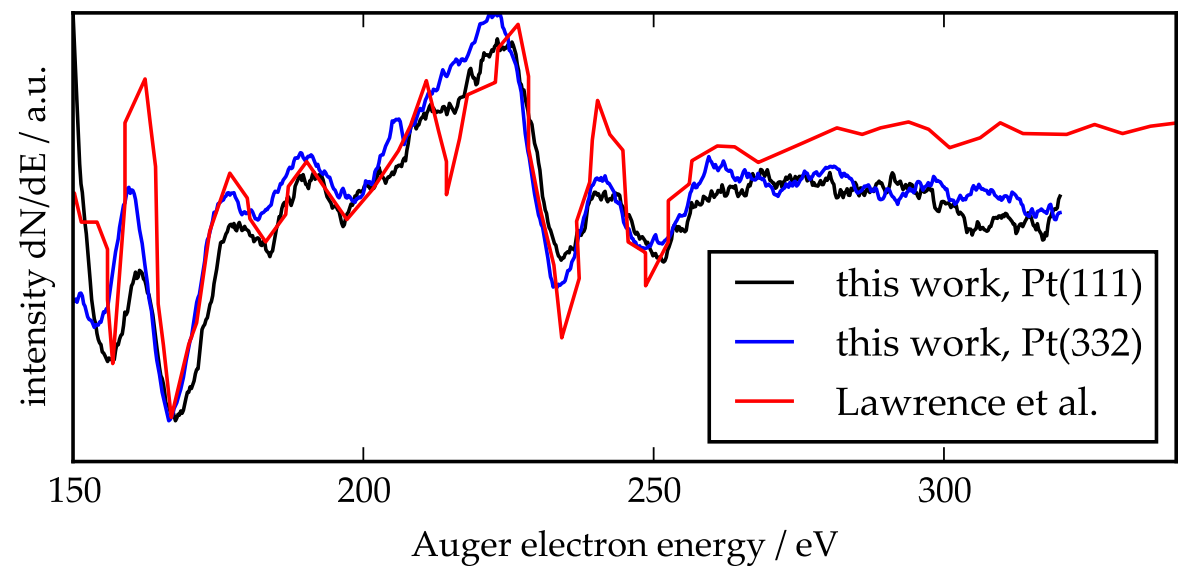

Figure 2.5: Platinum Auger spectrum at $3 \mathrm{keV}$ : The black curve is our $\operatorname{Pt}(111)$ Auger spectrum after baseline subtraction and the blue curve is the one of our $\mathrm{Pt}(332)$ crystal. The red curve is a digitized spectrum from the Handbook of Auger Electron Spectroscopy [12].

The major impurities present in platinum crystals according to Musket et al. are carbon $(272 \mathrm{eV})$, calcium $(291 \mathrm{eV})$ and phosphorus $(120 \mathrm{eV})$. Other impurities include sulfur, silicon, chlorine and oxygen. All of these impurities can be removed by sputtering. Carbon and calcium are found to segregate on the surface during heating, which can be detected in the Auger spectrum. 
We initially saw a strong carbon peak in the Auger spectrum after annealing. We thus performed over 100 cycles of annealing, sometimes in the presence of $10^{-6}$ Torr $\mathrm{O}_{2}$, followed by sputtering. During the annealing process carbon segregated from the bulk to the surface, where it was removed by reaction with oxygen or by sputtering. As the carbon peak did not reappear after this many cleaning cycles, I conclude that the carbon impurity in the bulk or at least near the surface of our platinum crystal must be strongly reduced.

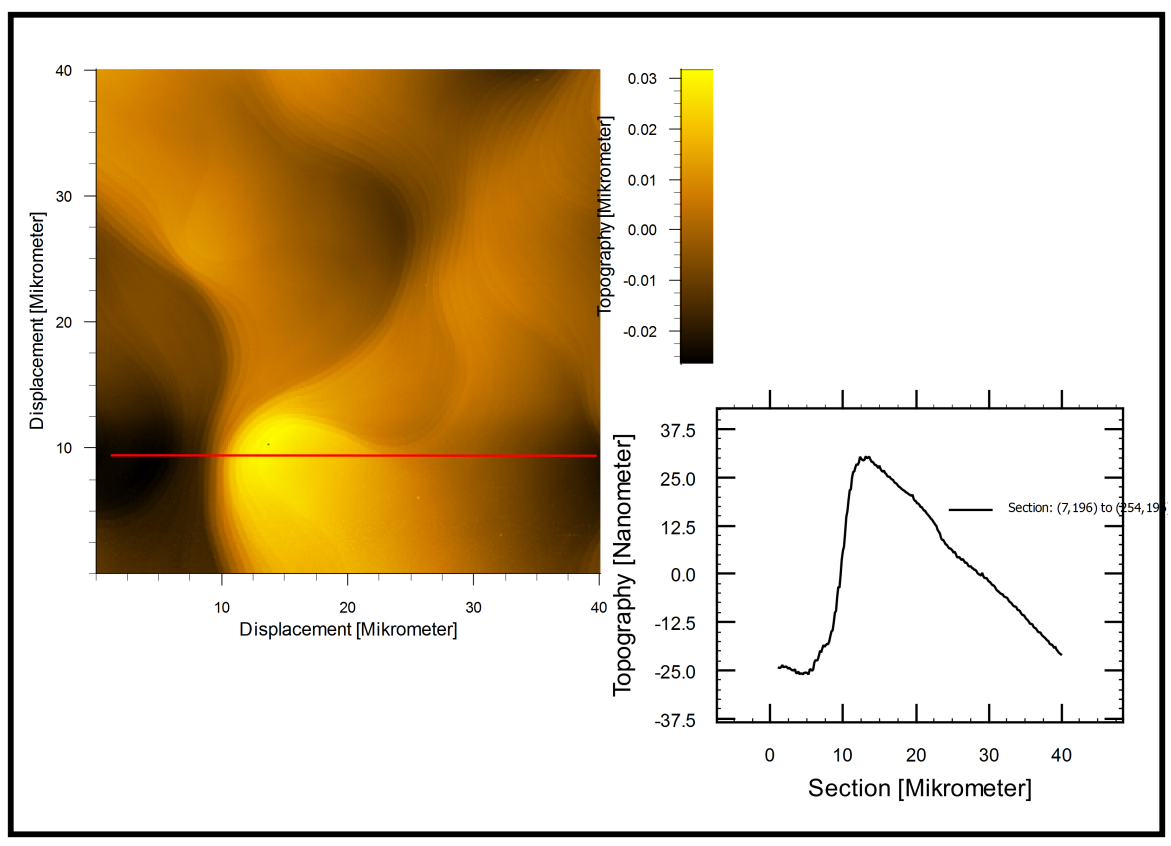

Figure 2.6: A 40x40 $\mu \mathrm{m}$ AFM picture of our $\mathrm{Pt}(111)$ crystal. A histogram of the topography along the red line is shown as an insert.

An atomic force microscopy (AFM) image has been recorded for $\mathrm{Pt}(111)$ to estimate the surface roughness, see figure 2.6. The AFM picture was provided as is by Philipp Buchsteiner of the Wenderoth group in the physics faculty, Georg-August University, Göttingen.

From the insert we can estimate a height displacement of $2 \times 50 \mathrm{~nm}$ up and down along the $40 \mu \mathrm{m}$ section. With a Pt-Pt bond length of $3 \AA$ this corresponds to $2 \times 167$ steps over a length of $40 \mu \mathrm{m}\left(1.3 \times 10^{5}\right.$ atoms $)$. Along one direction we estimate the step density to be $0.25 \%$.

In addition to the atomically flat $\operatorname{Pt}(111)$ crystal a stepped $\operatorname{Pt}(332)$ crystal was used. The (332) surface consists of 5-atom wide (111) terraces separated by a step, as shown in figure 2.7 . One out of six platinum atoms $(16.7 \%)$ on this surface are on steps.

\subsection{MOLECULAR BEAM GENERATION}

Well-defined molecular beam properties are a necessity in any beamsurface experiment. The focus in state-to-state scattering experiments 


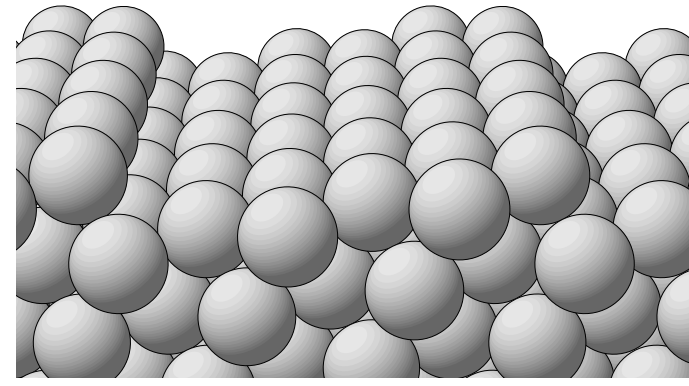

(a) $\mathrm{Pt}(332)$ step side view

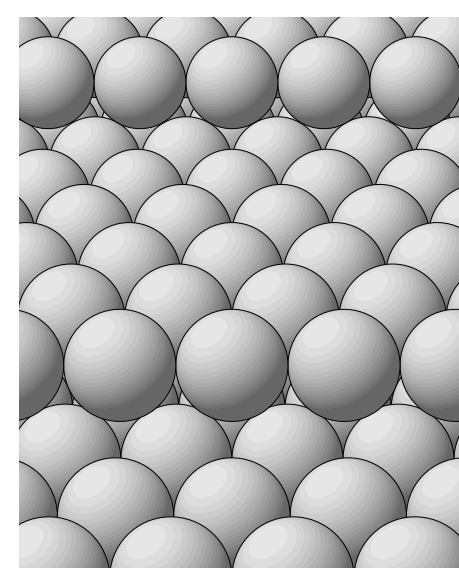

(b) $\operatorname{Pt}(332)$ step front view

Figure 2.7: Platinum surface structure: The $\mathrm{Pt}(332)$ has one step site per 5 terrace sites, i. e. a step density of $16.7 \%$. The step type is a (111)step.

mostly lies on efficient cooling of internal states during the supersonic expansion leading to controllable velocities and high speed ratios. The key factors in experiments on the kinetics at surfaces are high number densities and short beam pulses. All of these attributes are routinely obtained using pulsed supersonic beams.

An in-depth explanation of the supersonic expansion, cone-skimmer distance considerations resulting from the Mach disk position, etc. can be found in G. Scoles' Atomic and Molecular Beam Methods [13].

During the experiments at Beamer II, two different kinds of valves were used: A solenoid-actuated valve and a home-built piezoelectric disk-translator valve.

The solenoid valve performed well after fine-tuning the voltage and duration of the driving pulse. However, high backing pressures $(>8$ atm) were necessary and most importantly the solenoid valve was not meant to run faster than $20 \mathrm{~Hz}$. At higher repetition rates changes to the pulse profile were seen, most likely due to excess heat. In the CO-oxidation experiments high repetition rates of up to $333 \mathrm{~Hz}$ were indispensable to keep the duration of the experiments feasible.

The piezo valve shown in figure 2.8 is composed of a main body (e) with gas inlet (f) and a baseplate (d) holding the disk translator. The piezo disc translator works as follows: The back of the lead zirconate titanate $\left(\mathrm{Pb}\left[\mathrm{Zr}_{\chi} \mathrm{Ti}_{1-\chi}\right] \mathrm{O}_{3}\right)$ (PZT) crystal (a) is glued to the $0.6-0.75 \mathrm{~mm}$ thick steel membrane (b) with conductive silver epoxy. When a negative voltage between $500-1300 \mathrm{~V}$ is applied to the front of the piezo crystal it contracts radially [14] and the radial shear between piezo crystal and the steel membrane causes the membrane to curve. The combination acts as a bimorph. The stamp (c), which is screwed to the steel membrane, retracts to the right. The O-ring on the tip of the stamp sealing the nozzle orifice $(\mathrm{g})$ retracts as well and allows the gas 
For optimal prestress screw in the stamp until the O-ring barely seals, then apply another quarter turn.

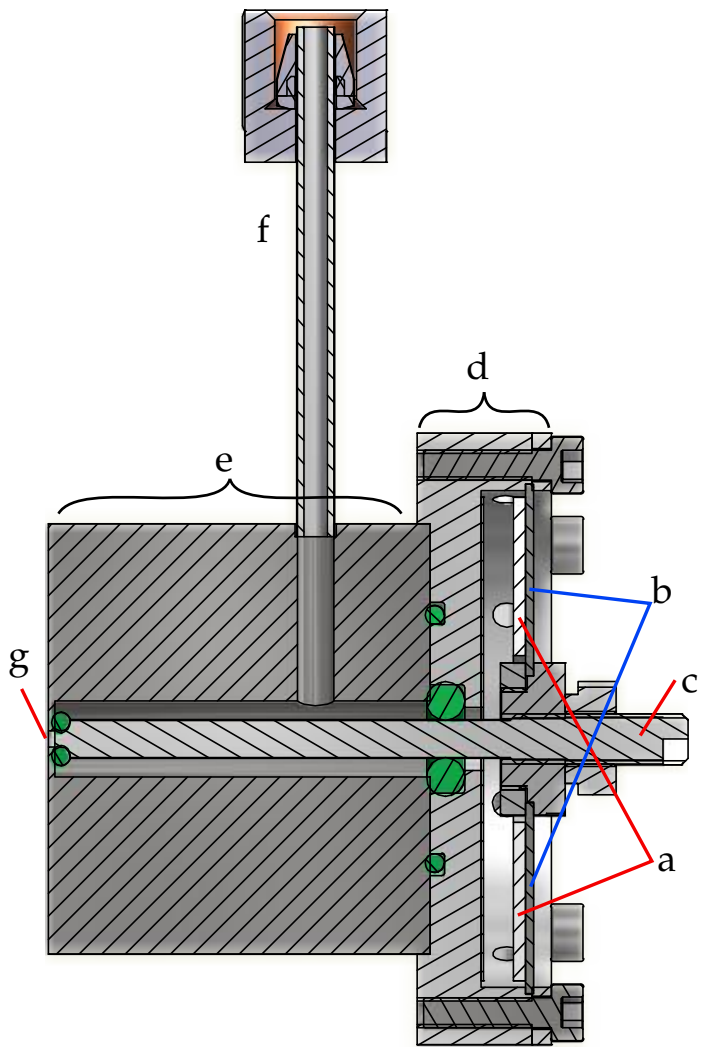

Figure 2.8: Drawing of the home-built PZT valve. The functional principle and labels are explained in the text.

in the volume surrounding the stamp in the main body to expand through the orifice.

The home-built piezoelectric valve is based on the design of Davis et al. [14], which is a refined version of the original design by Proch and Trickl [15]. Davis et al. unsuccessfully tried to use two stacked PZT crystals to generate a stronger driving force. We ordered thicker, custom-cut PZT crystals provided by Physik Instrumente Ceramic $\mathrm{GmbH}$, Thüringen, Germany to generate the high driving force, but circumvent the problems that arise when two PZT crystals are used.

The performance after carefully adjusting the prestress of the sealing O-ring and the driving pulse voltage and duration was competitive to the solenoid valve. 20-30 $\mu$ sWHM pulse duration were achieved with e.g. a $5 \% \mathrm{CO}$ in He mix with 5 bar stagnation pressure. Running several hours a day at up to $333 \mathrm{~Hz}$ for a year with minimal maintenance suggests an outstanding durability of over 1 billion pulses. 


\subsubsection{REMPI laser setup}

A Sirah Precision Scan Double Dye laser pumped by a Spectra-Physics Quanta-Ray Pro pulsed Nd:YAG laser can provide two frequency-tunable ns-pulses simultaneously. The first amplification unit in the double dye laser provides the fundamental wavelength with a tuning range of $400-920 \mathrm{~nm}$ ( 1800 lines $/ \mathrm{mm}$ grating, $0.1 \mathrm{~cm}^{-1}$ linewidth @ $625 \mathrm{~nm}$, up to 30\% efficiency). The second unit can be switched between providing the fundamental wavelength, with similar characteristics, or using second harmonic generation (SHG), third harmonic generation (THG) or sum frequency mixing (SFM) with $355 \mathrm{~nm}$ to generate high-energy photons. The second unit was used for all resonanceenhanced multi photon ionization (REMPI) detection schemes in this work.

For experiments using the double dye laser, the timing of all instruments is controlled by a BNC Model 575 digital pulse generator. The delay generator must be run on an internal clock with $10 \mathrm{~Hz}$, which is the recommended frequency for the Quanta-Ray pump laser.

\subsubsection{Strong field ionization}

For some molecules such as water or carbon dioxide REMPI is difficult. For these systems we instead focused high intensity fs-laser pulses to do non-resonant strong field ionization.

Initially we used a Clark-MXR CPA-Series $\mathrm{kHz}$ Ti:Sapphire laser which was installed in the laboratory across the hallway. Two periscopes and a safety tube connecting the two rooms across the hallway were installed to guide the laser to the Beamer II apparatus. The long distance sometimes caused instability of the laser alignment, presumably due to air circulation or pressure changes - the ventilation in the laboratory with the fs-laser was set to keep the room below atmospheric pressure to keep chemical fumes inside, while the ventilation in the Beamer II lab was set to keep the room pressurized to keep out dust. The power of the laser measured at the exit was about $0.8 \mathrm{~mJ}$ per pulse, which dropped to $0.5-0.6 \mathrm{~mJ}$ after the 8 mirrors of the beam line. The laser intensity provided was still sufficient for strong-field ionization of $\mathrm{CO}_{2}$ or $\mathrm{H}_{2} \mathrm{O}$.

In February 2017, a new high-power fs-laser (Coherent Astrella, 1 $\mathrm{kHz}$ Ti:Sapphire, up to $6 \mathrm{~mJ}$ per pulse in $<100 \mathrm{fs}$ ) was installed in the Beamer II laboratory. The superior day-to-day stability of the beam alignment compared to the other fs-laser reduced the time spent on preparation significantly. For molecules with a high ionization potential such as $\mathrm{CO}_{2}$ the increase in power greatly increased the ionization efficiency. This allows the measurement of systems where the flux is
Both laser pulses were used in a resonant four-wave mixing experiment [16], which is not presented in this thesis. 
very low. The higher signal-to-noise ratio allows the measurement range to be extended to conditions where slower kinetics are present, i.e. lower temperatures and concentrations.

\subsubsection{Trigger setup}

In this section I briefly describe how the various instruments in the lab are controlled. Please note that some terms used here (delayed extraction, $\mathrm{MCP}$, phosphor screen, etc.) and the imaging setup in general are introduced later in chapters 3 and 4 .

When using the nanosecond dye laser the BNC delay generator uses its internal clock as the master trigger. The relative timing of the various trigger pulses is illustrated in figure 2.9. The scan program

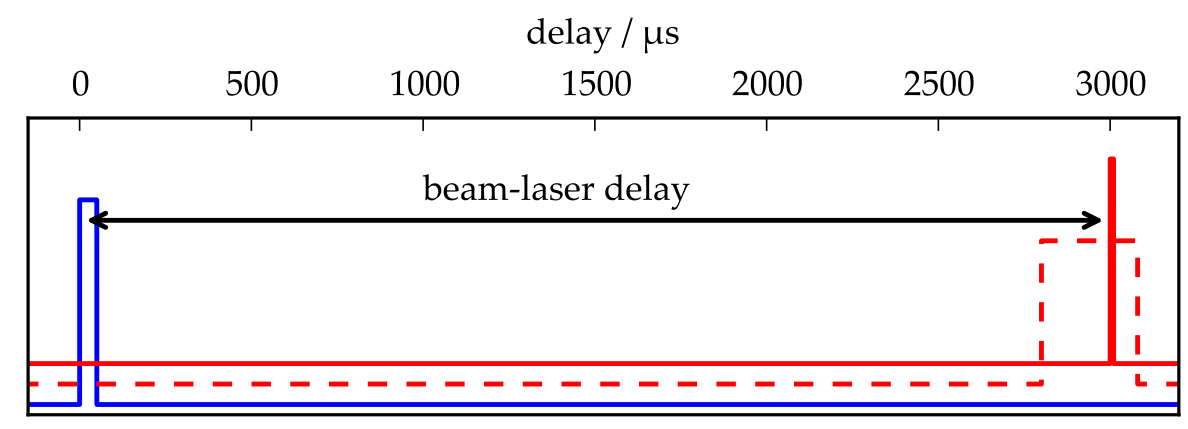

\begin{tabular}{|c|c|c|}
\hline $\begin{array}{ll}\text { - } & \text { molecular beam } \\
\text { - laser pulse }\end{array}$ & $\begin{array}{ll}-- & \text { flashlamps } \\
- & \text { repeller pulse }\end{array}$ & $\begin{array}{ll} & \text { MCP gate } \\
\text { - - } & \text { phosphor gate }\end{array}$ \\
\hline
\end{tabular}

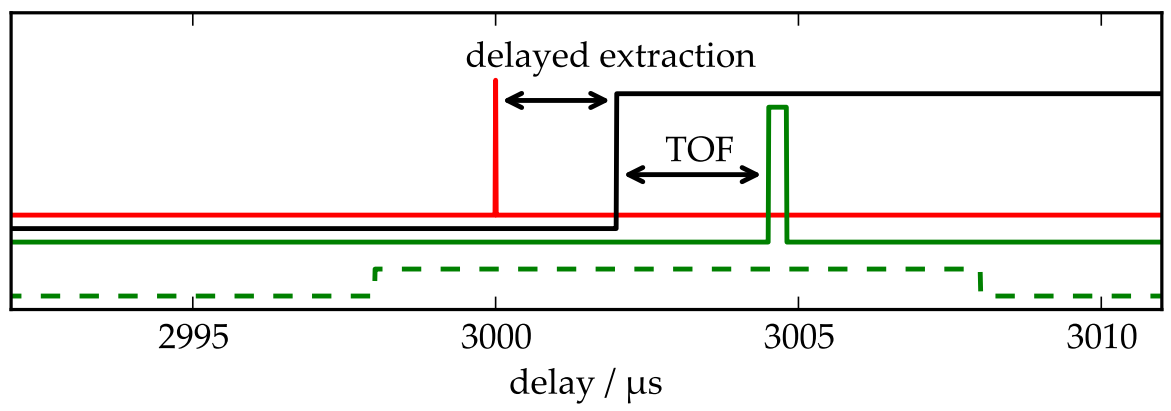

Figure 2.9: Relative position of the various trigger pulses for the ns-laser: The upper panel shows the relation between molecular beam pulse trigger and the laser, the lower panel shows the various pulses used in the detector gating.

(LaVision, Davis 8) controls the delay between the molecular beam and the laser Q-switch. The additional triggers when using a pulsed detector setup, i.e. the repeller pulse and the phosphor and $\mathrm{MCP}$

The advantages of pulsed detection are introduced in section 4.1 .2 on page 40. gates, are all defined relative to the Q-switch.

Both fs-laser run on an internal clock governed by the $80 \mathrm{MHz}$ resonator and cannot be synced to an external trigger. We therefore use the Q-Switch TTL signal of the Ti:Sapphire amplifier as the master trigger. The TTL signal of the Q-Switch is emitted at the time the laser 
pulse leaves the fs-laser, therefore we can not trigger the molecular beam valve prior to this pulse. To circumvent this problem, one must precisely determine the periodicity of the laser and use a later laser pulse following the master trigger for the experiment, as outlined in figure 2.10.

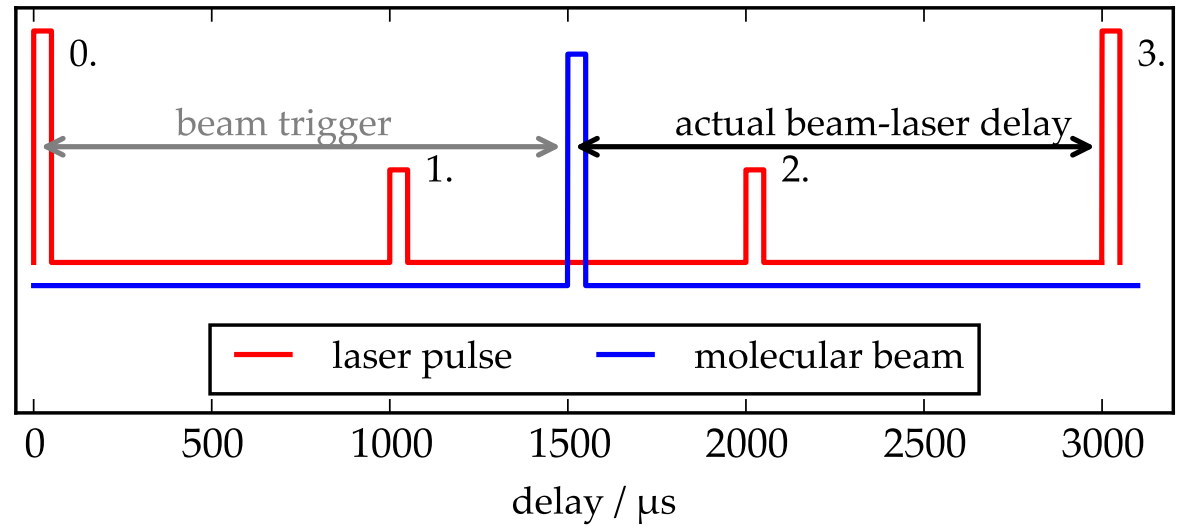

Figure 2.10: Trigger setup for the fs-laser: The TTL signal emitted at the zeroth laser pulse (red, dashed) is used as the master trigger. The molecular beam (blue, solid) is triggered with respect to this, indicated by the grey arrow. The ion optics and the detector are pulsed exclusively at the third laser pulse.

Since the Davis 8 software can only scan positive values, the molecular beam is controlled relative to the zeroth laser pulse. The actual beam-laser delay (black arrow) is then calculated by subtracting the beam trigger (grey arrow) from the delay between zeroth and third laser pulse.

As a result of this setup, which uses 4 laser shots per cycle, the maximum repetition rate for the molecular beam and the ion optic$\mathrm{s} /$ detector is $250 \mathrm{~Hz}$. The beam-laser delay can be scanned over $3 \mathrm{~ms}$. If a higher scan range is needed or if the time between pulses is too short to ensure a clean surface between each pulse, this setup can be adjusted to use the $5^{\text {th }}$ or 1oth laser pulse.

The second molecular beam, which was used to dose $\mathrm{O}_{2}$ in the CO-oxidation experiments, is controlled by another delay generator. This delay generator (Stanford Research Systems, DG 535) uses the fs-laser master trigger as input and acts as a frequency divider. The $\mathrm{O}_{2}$ beam was generated right after the master trigger to ensure that the beam pulse is gone before the next laser shot. Due to the high gas load in the source chamber and the strain on the PZT valve the highest repetition rate used was $333 \mathrm{~Hz}$.

\section{REFERENCES}

[7] R. G. Musket, W. McLean, C. A. Colmenares, D. M. Makowiecki, and W. J. Siekhaus. "Preparation of atomically clean surfaces
The periodicity was determined with a fast photodiode. The correct timing between laser pulse and repeller gate pulse is crucial in ion imaging as it affects the measured speeds.

For more information on the two-beam trigger setup see Appendix C.2. 
of selected elements: A review." In: Applications of Surface Science 10.2 (Jan. 1982), pp. 143-207. DOI: 10. 1016/0378-5963 (82) 90142 - 8 (cit. on p. 10).

[8] William Hallowes Miller. Treatise on Crystallography. London: Cambridge University Press, 1839 (cit. on p. 10).

[9] Lise Meitner. “Über die beta-Strahl-Spektra und ihren Zusammenhang mit der gamma-Strahlung." In: Zeitschrift für Physik A Hadrons and Nuclei 11.August (1922), pp. 35-54. Dor: 10 . 1007/BF01328399 (cit. on p. 10).

[10] Pierre Auger. "Sur les rayons $\beta$ secondaires produits dans un gaz par des rayons." In: Comptes Rendus 180 (1923), pp. 169171 (cit. on p. 10).

[11] Michael Schwarzer. "Reaction Dynamics and Kinetics of CO Oxidation on $\operatorname{Pd}(111)$ using Ion Imaging." Bachelor Thesis. Georg-August-Universität Göttingen, 2017 (cit. on p. 10).

[12] Davis E. Lawrence, Noel C. MacDonald, Paul W. Palmberg, Gerald E. Riach, and Roland E. Weber. Handbook of Auger Electron Spectroscopy. 2nd ed. Eden Prairie, Minnesota: Physical Electronics Industries, 1976 (cit. on p. 11).

[13] Giacinto Scoles, Davide Bassi, and Udo Buck. Atomic and Molecular Beam Methods. Vol. 1. 1988 (cit. on p. 13).

[14] David L. Proctor, Daniel R. Albert, and H. Floyd Davis. "Improved piezoelectric actuators for use in high-speed pulsed valves." In: Review of Scientific Instruments 81.2 (2010). DOI: 10. 1063/1.3309777 (cit. on pp. 13, 14).

[15] D. Proch and T. Trickl. "A high-intensity multi-purpose piezoelectric pulsed molecular beam source." In: Review of Scientific Instruments 60.4 (1989), pp. 713-716. DOI: 10.1063/1.1141006 (cit. on p. 14).

[16] Alice F. Schmidt-May, Monika Grütter, Jannis Neugebohren, T. N. Kitsopoulos, Alec M. Wodtke, and Dan J. Harding. "Rotationally Resolved Vacuum Ultraviolet Resonance-Enhanced Multiphoton Ionization (VUV REMPI) of Acetylene via the $\tilde{\mathrm{G}}$ Rydberg State." In: The Journal of Physical Chemistry A 120.27 (July 2016), pp. 5399-5407. Dor: 10 . 1021/acs . jpca . 6 b02477 (cit. on p. 15). 


\subsection{IMAGING TECHNIQUES}

Ion imaging of gas-phase reactions has become a well-established technique since its invention in 1987 by Chandler and Houston [17]. An introduction to the ion imaging technique and its further developments, most notably velocity-map imaging (VMI) [18] and sliceimaging [19], as well as related problems such as the inverse Abel transformation $[20,21]$ and density-to-flux conversion is given in this chapter.

\subsubsection{Spatial imaging}

The spatial imaging technique preserves information of the position of the ion prior to acceleration by electric fields.

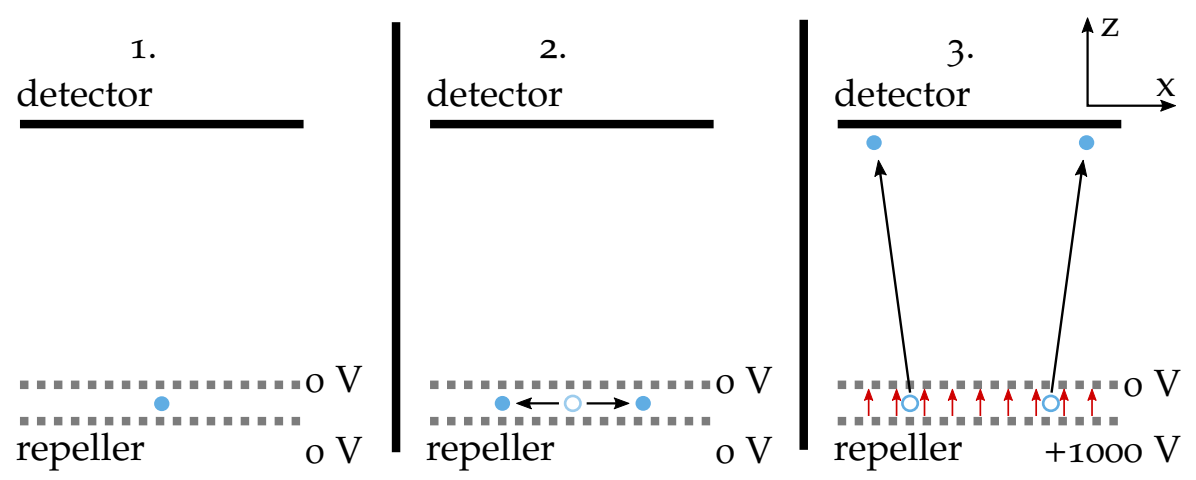

Figure 3.1: Spatial imaging sequence explained in three steps: 1 . Two ions with opposite velocities are produced between two grids on the left. 2. If the extraction pulse is delayed, the ions will fly along their initial direction in a field-free zone. 3. An electric field is pulsed and accelerates the ions onto the detector on top. Their position and velocity along the $\mathrm{x}$-axis is preserved.

Consider two ions produced by photofragmentation at the same position between two grids, as indicated by the blue dot in figure 3.1 on the left. The polarization of the laser causing the photofragmentation The ions are not meant to be produced by the same event. is parallel to the grids and the fragmentation recoil is along this axis, as indicated by the arrows. The grids are grounded and the ions move along their initial direction in a field free zone, as shown in the middle panel. When the repeller grid is pulsed to e.g. $+1000 \mathrm{~V}$ in the third panel, the ions are accelerated upwards by the homogeneous electric field (red arrows), where they impinge on the detector. The movement of the ion along $x$ is not changed by the perpendicular electric 
field. The spatial position of the ions is therefore preserved through the extraction, and the displacement along $x$ during the flight time to the detector is added to the position. The separation of the ion spots on the detector, as shown at the top in frame 3 of figure 3.1, is equal to $v_{\text {rel }} \times t_{\text {total }}$, where $v_{\text {rel }}$ is the velocity of the ions relative to each other along $x$ after photofragmentation and $t_{\text {total }}$ is the time between ionization and impact on the MCP. The separation can be increased by pulsing the repeller a short time $t_{\mathrm{DE}}$ after the laser pulse. The total time is $t_{\text {total }}=t_{\mathrm{TOF}}+t_{\mathrm{DE}}$, where $\mathrm{t}_{\mathrm{TOF}}$ is the time-of-flight after pulsing the repeller. This technique is called delayed extraction.

While spatial imaging allows a large range of applications and interpretation of the resulting ion image is straightforward, the speed resolution is limited to the spatial extent of the ion source volume. This limitation shall now be discussed using the experimental setup of the original paper of Chandler and Houston as shown in figure 3.2. A molecular beam of $\mathrm{CH}_{3} \mathrm{I}$ in He passes through a narrow hole in

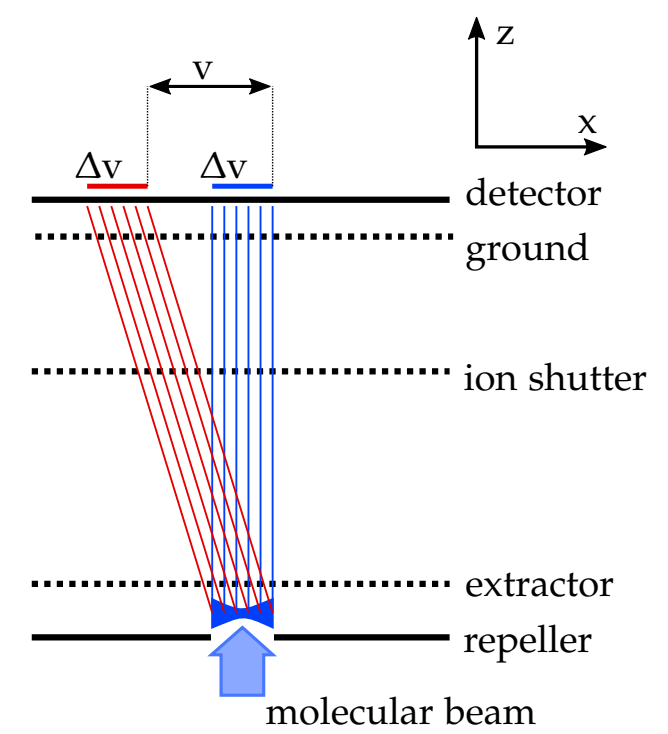

Figure 3.2: Experimental setup of the original ion imaging experiment by Houston and Chandler. The velocity resolution $\Delta \mathrm{v}$ along the $\mathrm{x}$ axis is limited by the focus length of the laser.

the repeller plate. A $266 \mathrm{~nm}$ unfocused laser along the x-axis initiates the photofragmentation of $\mathrm{CH}_{3} \mathrm{I}$. A second counterpropagating laser ionizes the $\mathrm{CH}_{3}$ fragment using a $2+1$ REMPI scheme. The focus of the REMPI laser is indicated by the hourglass shape. A continuously applied electric field of $200 \mathrm{~V}$ between repeller plate and extractor grid immediately accelerates the ions towards the detector. After the $\mathrm{CH}_{3}^{+}$-ions have passed the ion shutter, a voltage of $+250 \mathrm{~V}$ is applied to repel slower $\mathrm{CH}_{3} \mathrm{I}^{+}$-ions. As indicated on top of the detector, any velocity $\mathrm{v}$ of the ions along the $\mathrm{x}$-axis has an uncertainty $\Delta \mathrm{v}$, equal to the overlap of laser focus and molecular beam as indicated by the hourglass shape. The velocity uncertainty along the $y$ - 
axis (not shown) should be on the order of the focus width, i.e. about $100 \mu \mathrm{m}$. Velocity measurements in experiments using spatial imaging typically have uncertainties $\Delta \mathrm{v} / \mathrm{v}$ on the order of $15-20 \%$ [22]. In a two laser experiment the resolution along the $x$-axis can be improved by having the two lasers intersect at an angle of $90^{\circ}$. While this is applicable to some photofragmentation or crossed beam experiments, not all experiments use two lasers or offer the required geometry.

\subsubsection{Velocity map imaging}

A way to circumvent the velocity resolution limit of ion imaging due to the spatial extent of the ion source volume was established by Eppink and Parker [18] in 1997. VMI makes use of an inhomogeneous electric field, which acts as an ion lens. In analogy to a lens focusing light, the ion lens focuses all ions with the same velocity onto the same point on the detector, as indicated by the converging lines in figure 3.3. The focus point of ions with an additional velocity component $\mathrm{v}$ along the $\mathrm{x}$-axis (red lines) is shifted by an offset proportional to $\mathrm{v}$ compared to the focus point of zero velocity ions (blue lines).

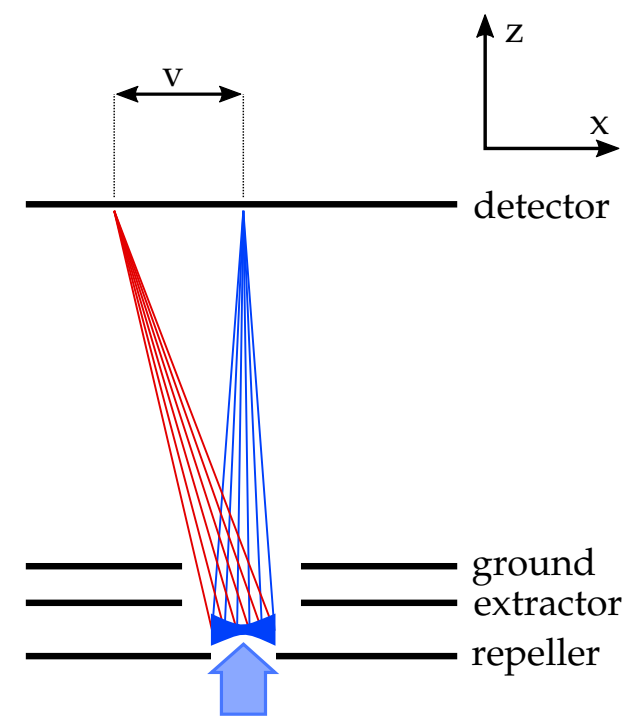

Figure 3.3: VMI setup of Eppink and Parker. The open ring electrodes generate a curved electric field. All ions with the same velocity are focused onto the same point, irrespective of their spatial position at ionization.

Since only the velocity component of the ions is preserved through the extraction, the VMI technique is especially useful when ions are generated in a large area. Hereby a spatial spread due to e.g. the length or width of a laser focus or molecular beam can be eliminated, resulting in resolutions $\Delta \mathrm{v} / \mathrm{v}$ on the order of 2-5\% [22].

The inhomogeneous electric field can be generated by either using open electrode geometries as shown in figure 3.3 or by adding

Note that velocity includes both speed and direction.
The diameter of the ion source should be much smaller than the ion lens to avoid aberrations. 
The parent mass is used because these ions have not undergone fragmentation and have no recoil velocity.

A ring, as used here, has a finite width $\Delta r$ while a circle is considered infinitely thin.

The polar angle $\vartheta$ is the angle between a product vector and the rotational axis, see figure 3.6 . a second ring electrode (einzel lens) between the extractor and the ion shutter in figure 3.2. When using the open electrode design, a voltage of e.g. $+1000 \mathrm{~V}$ is applied to the repeller plate and a smaller voltage of e.g. $+300 \mathrm{~V}$ to the extractor plate to generate the proper inhomgeneous electric field. The procedure to calibrate the voltage ratio depends on the available experimental setup. For the experimental setup with open electrodes shown above the first step would be to create ions with zero or well-defined velocity, e. g. from a background gas or the parent mass of a molecular beam. The focusing is then tested by moving the interaction region with the laser focus - if VMI conditions apply, the ion spots will not move. For an experimental setup using two closed grids and an einzel lens on top, the calibration can be done by comparing the velocity map images at different extraction delays - they should be independent of the extraction delay.

\subsubsection{Abel transform}

The quantities of interest in gas phase experiments such as photofragmentation or photoelectron spectroscopy are the velocities and the related intensities. In principle both spatial and velocity map imaging detect all ions, irrespective of their velocity. Let us consider a process generating an isotropic (spherical) product distribution, as depicted by the sphere in figure 3.4. The resulting ion image is the projection of the sphere onto the $2 \mathrm{D}$ screen in the $\mathrm{xy}$-plane. The projection of a sphere onto a $2 \mathrm{D}$ screen yields a disc with varying intensity along the radius $r$ (red line), this projection is known as Abel transform [23]. The distribution along the radius is represented by the increasing density of circles with increasing radius. The intensity along the radius $r$ (red line in figure 3.4) is similar to the projection of a ring onto a line, which is illustrated in figure 3.5. The projection of a ring onto a line is described by the $2 \mathrm{D}$ Abel transform.

The projection of the $3 \mathrm{D}$ product distribution onto the detector is termed crushed image in the gas phase imaging community. The maximum velocity of a single ring in a crushed image is straightforward: After finding the center, one can either sum the intensity along a line through the center (a histogram with a finite thickness along a line) or integrate the intensities in a sector $r \times d \vartheta$ as a function of radius $r$. The radius is proportional to the speed. If multiple rings are present, however, the peaks of a smaller ring can easily be concealed by the intensity inside a larger ring. This effect becomes more important if the rings have a large width (are not sharp), e.g. due to the spatial extent of the source.

The comparison of the intensity of two different velocity components is not as simple. For a single process and under the assumption of cylindrical (rotational) symmetry with the axis of rotation along 


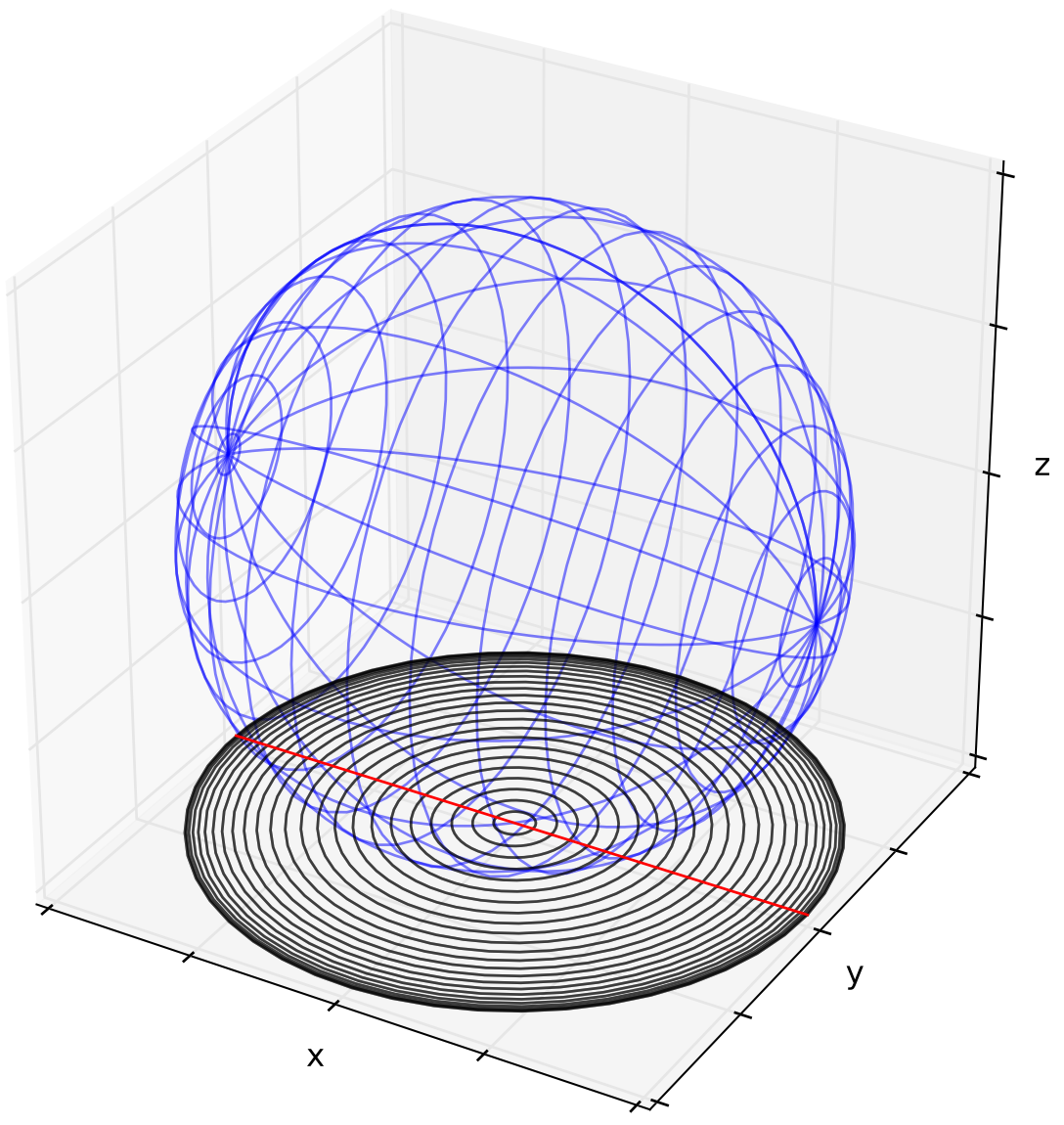

Figure 3.4: $3 \mathrm{D}$ Abel transform: An isotropic spherical distribution (blue wireframe) is projected onto the xy-plane. The resulting pattern is a disc (black circles) with varying intensity along the diameter (red line). The disc and its intensity is represented by a series of circles, with smaller distance between circles at larger radii.

$x$ in the imaging plane, as shown in figure 3.6, we can reconstruct the $3 \mathrm{D}$ intensity from the intensity distribution along the outer circle. This distribution along the polar angle $\vartheta$ is depicted by the blue circle in figure 3.6. The $3 \mathrm{D}$ intensity at a polar angle $\vartheta$ is calculated by integrating the intensity at each point along this ring over the azimuthal angle $\varphi$, with

$$
\mathrm{I}^{3 \mathrm{D}}(\vartheta)=\int_{0}^{2 \pi} r \sin (\vartheta) \mathrm{d} \varphi=2 \pi \mathrm{r} \sin (\vartheta) .
$$

This operation can be imagined by rotating the blue circle around the axis of rotation (black arrow), resulting in the red circles. The $3 \mathrm{D}$ intensity at an angle $\vartheta$ is proportional to the circumference of these red circles.

In order to perform this calculation, we need the intensity along the blue circle in figure 3.6. For an ion image with a single product velocity and therefore a single ring, this distribution can be calculated by integrating over $r d r \vartheta d \vartheta$. For an ion image with multiple rings the

Note that I chose the axis of rotation to be along $x$ to be consistent with the terminology in this work. 


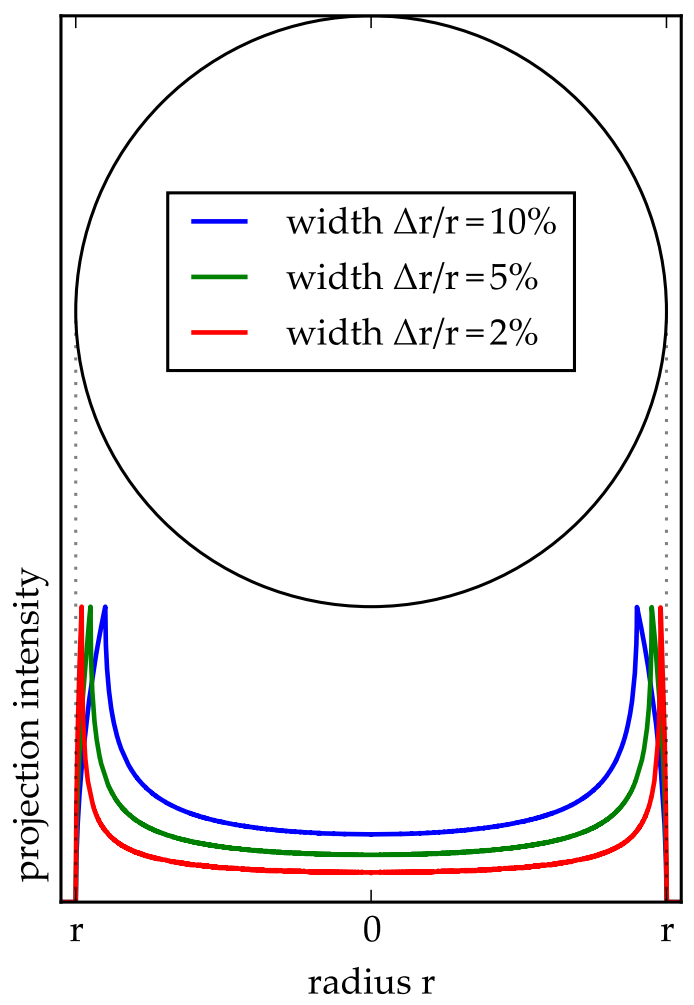

Figure 3.5: Projection in ion images: The ring in the $\mathrm{xz}-$ plane with radius $r+\Delta r$ at the top is projected onto the $\mathrm{x}$-axis assuming three different widths $\Delta r$ as given in the legend. The resulting intensity distribution along the $\mathrm{x}$ axis shows two sharp peaks at the radius $r$. The ratio of the peak intensity to the intensity in between increases with smaller linewidths $\Delta r$. The ring is equivalent to a slice of the spherical ion distribution (figure 3.4) along the xz-plane.

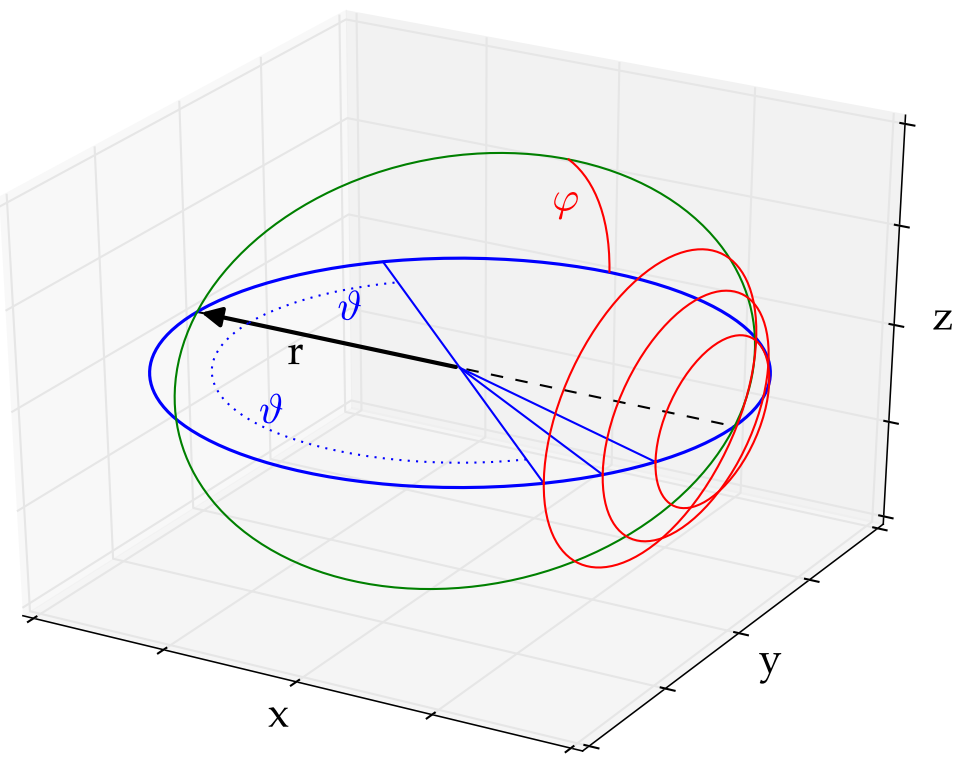

Figure 3.6: Polar coordinate system: $0<\vartheta<\pi$ is the polar angle between the axis of rotation and a vector. $0<\varphi<2 \pi$ is the azimuthal angle in the yz-plane orthogonal to the axis of rotation. 
inner intensity of the large ring must be subtracted before integrating over the smaller ring. This operation can be performed using the inverse Abel transform. This mathematical operation basically keeps the intensity at the maximum radius and removes any signal of this process at smaller radii. This allows for further identification of smaller rings. There are different algorithms to reconstruct $3_{3} \mathrm{D}$ images from crushed images. Depending on the algorithm the inverse Abel transform is very sensitive to the position of the center supplied to it and to any noise in the data. The superior approach would be to measure slice images from ion spheres directly, see section 3.1.4.

Another important factor when comparing the intensities of different velocity components is the different volume the ions occupy based on their distance to the center. Figure 3.7 shows a slice ion image generated by a photofragmentation process. Slice images contain

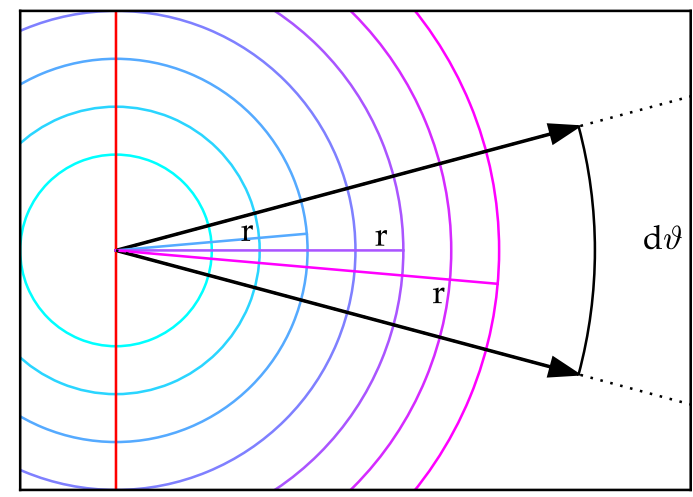

Figure 3.7: A laser (red) photolyzes a molecule and ionizes the product. All ions show an isotropic distribution with different velocities (colored circles). A sector d $\vartheta$ for integration is marked by two arrows.

ions fragmenting in the xy-plane exclusively. The resulting image is a number of rings with different intensities. The different velocities are due to the internal energy of the molecule prior to fragmentation. In order to assess the internal energy distribution, we need to measure the relative intensity of the different products. For an isotropic distribution this is straightforward - we only need to integrate the intensity in a sector as a function of the radius $r$. For a ring with faster velocities the ions spread over a larger area in the ion image. This "dilution" is proportional to the radius $r$ of the ring. Since the integration area along the sector increases linearly with the radius, these two effects cancel out. If we were to calculate the intensity along a line with fixed absolute width (histogram), we would integrate over a larger $\mathrm{d} \vartheta$ at small radii and over a smaller $\mathrm{d} \vartheta$ at large radii. This would bias the intensities.

Lastly, it is important to know whether the detected signal is equivalent to the density or the flux. In a well-designed gas phase imaging experiment the photolysis laser's focus volume is equally small as or smaller than that of the ionization laser. If ns-lasers are used, the movement of the fragments between photolysis and ionization is very small. In order to assess the effect of fly-out we consider an $\mathrm{H}$-atom with $1 \mathrm{eV}$ kinetic energy release. The $\mathrm{H}$-atom moves away

The internal energy of the ions is determined by the REMPI scheme. Any difference in velocity is due to the internal energy prior to fragmentation, see section 3.2.3. 
with $15000 \mathrm{~m} \mathrm{~s}^{-1}$, or $150 \mu \mathrm{m}$ in $10 \mathrm{~ns}$. For a laser focus with a diameter of $50-100 \mu m$, this is just enough to allow a number of $\mathrm{H}$-atoms to escape. For heavier atoms or molecules the fraction leaving the ionization volume is negligible. For a molecule as heavy as $\mathrm{N}_{2}$ and temporally overlapping lases pulses with a width of $<10$ ns the ionization is independent of the velocity and we detect the flux. Special care must be taken in experiments where the two laser pulses are temporally separated to avoid nonlinear effects such as multiphoton ionization (MPI).

In gas-phase experiments where the products are not generated by a short laser pulse but by a longer process, such as two crossed molecular beam pulses, the chance for the laser to ionize the product is proportional to its density. The larger rings in ion images will thus appear less intense. If the recorded image is a crushed image, one should first apply the inverse-Abel tranform to obtain the slice image. Afterwards, one could multiply the intensity at each pixel with its respective velocity. Since the ionization probability scales with $1 / v$, this would correct the ionization probability bias.

Another approach is to integrate the slice image intensity of a sector $d \vartheta$ along the radius $r$ and convert the radius (velocity) to energy. When transforming the signal from velocity to energy space using

$$
\mathrm{I}(\mathrm{E}) \mathrm{dE}=\mathrm{I}(v) \mathrm{d} v,
$$

one would multiply the signal $\mathrm{I}(v)$ with

$$
\frac{\mathrm{d} v}{\mathrm{dE}}=\frac{1}{\mathrm{~m} v} \text {. }
$$

Instead of first multiplying with $v$ and then dividing by $m v$, one can then drop the $y$-value transformation altogether and just transform the $\mathrm{x}$-values to energy.

\subsubsection{Slice imaging}

The idea of slice imaging [19] is to detect only those ions with velocities parallel to the imaging plane. For a gas phase process such as photodissociation of $\mathrm{N}_{2} \mathrm{O}$ [24] followed by REMPI detection of the product $\mathrm{N}_{2}$ molecule, this is accomplished as illustrated in figure 3.8:

A laser (green line) initiates photofragmentation and ionizes one of the resulting fragments. Due to the recoil, the ions (blue, red and ma-

Slice imaging is especially useful when dealing with non-spherical processes [22], but for simplicity a spherical one is assumed. genta dots) have isotropical velocity distributions. A field-free region allows the ion cloud to expand with its initial velocity distribution, which results in a spherical distribution as indicated by the two rings. Ions with velocities along $\mathrm{z}$ (red and blue) leave the middle of the extraction region and approach the extractor or the repeller, while ions with zero velocity along $\mathrm{z}$ (magenta) remain in the middle.

On the right side of figure 3.8 pulsed voltage is applied to the repeller and the ions are accelerated towards the MCP by the electric 
1 .
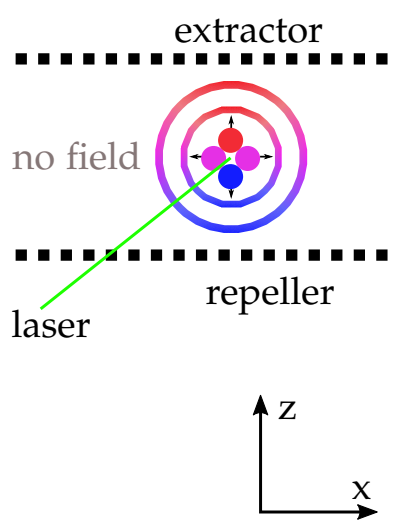

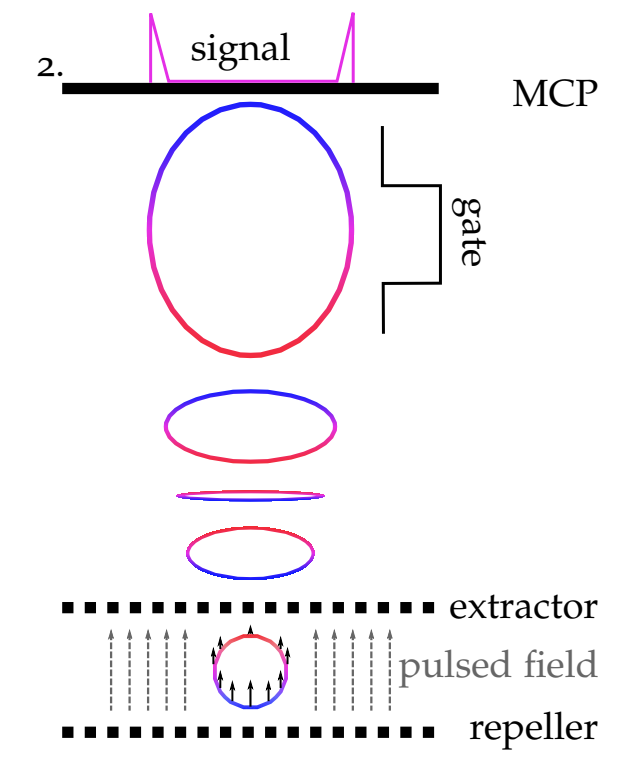

Figure 3.8: Slice imaging technique. Left: A laser pulse generates an isotropically expanding ion cloud. Right: A pulsed field is used to accelerate the ions towards the gated $\mathrm{MCP} /$ phosphor screen detector. The ions have a large TOF spread and the MCP gate is used to select molecules with zero initial velocity along $\mathrm{z}$, see text.

field. Ions near the repeller have a higher potential and attain more kinetic energy while ions near the extractor attain less kinetic energy. The ion cloud thus leaves the extraction region with different velocities along $\mathrm{z}$. The ion cloud transforms into a "cigar shaped" object on the way to the MCP. By gating the front MCP with a narrow pulse only the ions in a narrow time window are detected. When this window is chosen properly only the middle part of the ion cloud, i.e. molecules with zero initial velocity along $\mathrm{z}$ are detected. The resulting ion image shows a narrow ring for every product velocity without any intensity in the middle. This eliminates the need to perform an inverse Abel transform and makes the comparison of the intensities, as explained in the section above, straightforward.

\subsubsection{Event counting}

Event counting or centroiding is a technique which can improve the resolution of the imaging system by collapsing the ion spot size to a single pixel or even sub-pixel resolution. Typical ion spot sizes at our detector have a full width at half maximum (FWHM) of 6-10 pixels, as shown in figure 3.9. For the ion spot in the middle of the image at $\mathrm{x}=185$ and $\mathrm{y}=405$ the center can be determined by fitting two gaussian functions along $x$ and $y$. A unit intensity of e.g. 1 is then added to the event counting sum image at these coordinates. The correct determination and quantification is much more difficult for overlapping 


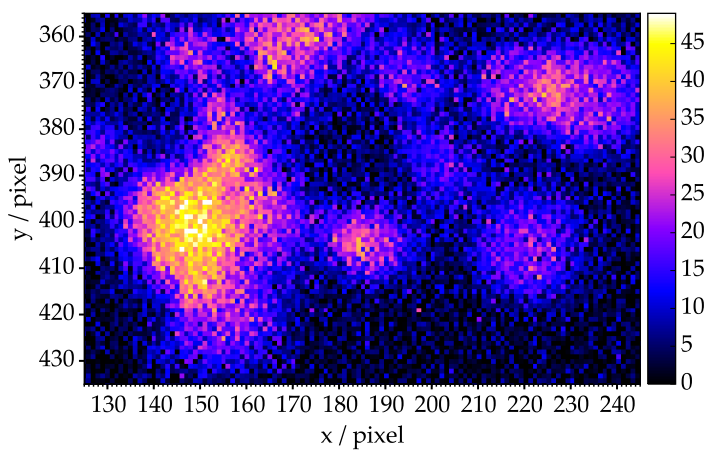

Figure 3.9: Ion spot size before event counting: The typical FWHM is about 610 pixels. Some ion spots are clearly distinguishable, others are overlapping.

ion spots. The ion spots at the center or the lower right are clearly separated from everything else, while two ion spots seem to overlap in the upper right corner. For the cluster of ion spots on the left it is impossible to identify single events. If this problem is ignored and the cluster is counted as a single event, the event counting image would constantly underestimate the number of events in regions with a high number of ion spots. For event counting it is therefore necessary to attain conditions with very few, well distinguishable ion spots per image - ideally combined with a high repetition rate of the experiment.

\subsection{GAS-PHASE IMAGING AT THE VMI MACHINE}

\subsubsection{Experimental setup of the VMI chamber}

The following experiment was carried out at a separate experimental setup we used during the construction of the Beamer II apparatus. I briefly describe this setup, the VMI chamber, in the section below. The setup consists of the main chamber, a large CF-200 6-way cross, and a source chamber with differential pumping stage. A cut through the chamber along the vertical xz-plane is shown in figure 3.10.

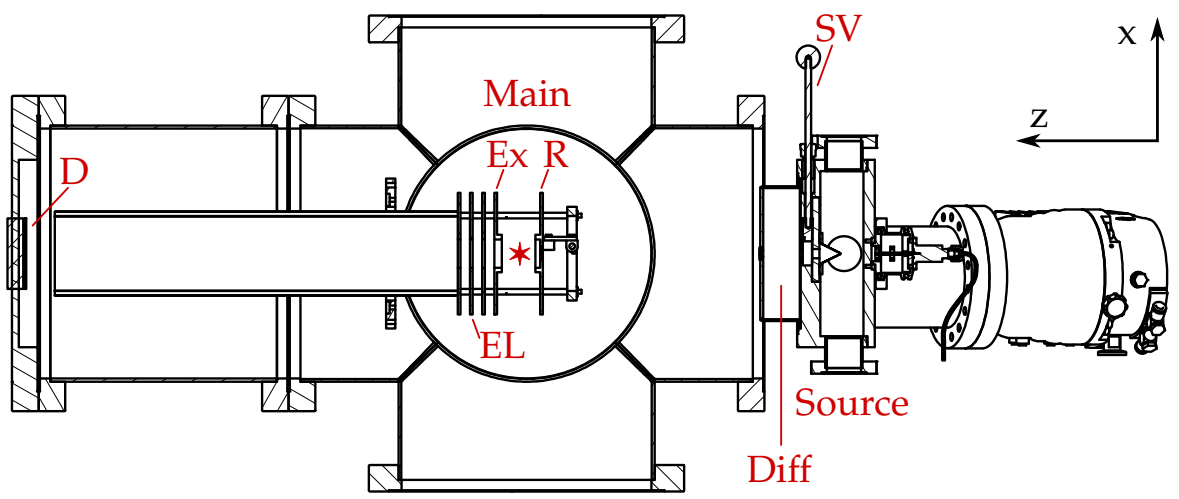

Figure 3.10: Side view of the VMI chamber: The slide valve is labeled ' $S V^{\prime}$, the repeller ' $R$ ', the extractor ' $E x$ ', the einzel lens ' $E L$ ' and the detector ' $\mathrm{D}$ '. 
A molecular beam is generated in the source chamber on the right and passes through a skimmer (Beam Dynamics Model 2), a slide valve (see figure 3.11, right side) and the differential pumping stage before entering the main chamber. The source chamber, the differential pumping stage, and the main chamber are pumped by maglev TMPs with $300 \mathrm{Ls}^{-1}, 300 \mathrm{Ls}^{-1}$ and $1000 \mathrm{Ls}^{-1}$, respectively.

The stack of vertical lines in the center of the main chamber represent the ion optics. The molecular beam passes through a hole in the repeller plate to the position of the laser access along the y-axis, as indicated by the red star between repeller and extractor plate. The repeller plate can be moved along $\mathrm{z}$ to allow optimization of the electric fields for velocity mapping [24]. Ions produced by the laser interaction are accelerated towards the left and pass the extremely fine extractor grid (100o lines-per-inch nickel grid by Precision Electroforming). Depending on the application an additional electric field between extractor grid and detector can be applied using the einzel lens, which is surrounded by two grounded electrodes. The flight path to the detector is shielded from interfering magnetic and electric fields by two stacked $\mu$-metal cylinders. The stacking also allows the total length of the shielding cylinder to be adjusted. A Helmholtz coil around the 6-way cross can be used to cancel residual magnetic fields in the ionization region when imaging photoelectrons.

The ions or electrons are detected by a Photonis $75 \mathrm{~mm}$ imaging detector system consisting of a single MCP and a fiber optic coupled P47 phosphor screen. The Imager E-lite camera (1626x1236 pixel) and customized Davis v8.o image acquisition softwar by LaVision are the same as used in the Beamer II setup.

Two pictures of the source region are presented in figure 3.11. A

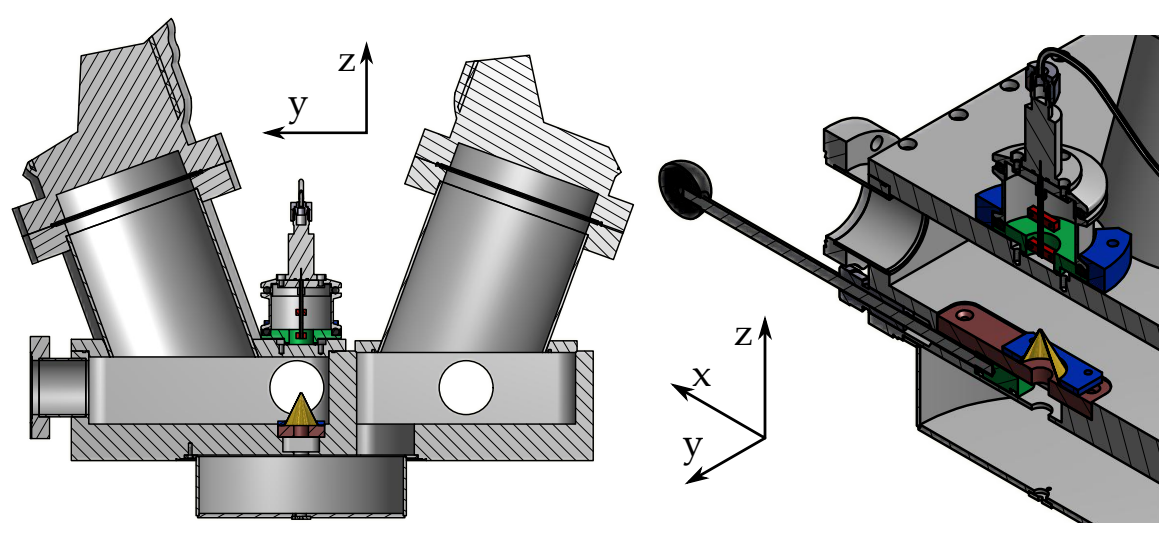

Figure 3.11: Source chamber: Left: Overview of the source chamber. Right: A cut along the xz-plane showing the hot nozzle setup, the differential pumping stage and the slide valve in between.

Parker General Valve Series 8 in conjunction with a $30 \mathrm{~mm}$ long $\mathrm{SiC}$ tube mounted to the exit is used to generate molecular beam pulses. The $\mathrm{SiC}$ tube can be heated by passing a current through two elec-

The $\mu$-metal cylinders were degaussed by moving them in a coil with initially strong, then decreasing current. 
trodes mounted on the outside of the tube. Although similar to P. Chen's flash pyrolysis source [25] this feature was not used to produce radicals but vibrationally hot molecules.

\subsubsection{Photoelectron spectroscopy of $\mathrm{CO}_{2}$}

In 2014 we tested the applicability and efficiency of $\mathrm{CO}_{2}$ REMPI schemes. The efficiency of the $3+1$ REMPI through the $3 p \sigma_{u}{ }^{1} \Pi_{\mathfrak{u}}$ state at $326.4 \mathrm{~nm}$, which offers the largest ionization cross section in that region [26], turned out to be too low for surface scattering experiments. However, we recorded a photoelectron image in the process, which shall be used to demonstrate the effect of the inverse Abel transform. The energy of the photoelectron depends on the internal energy of the corresponding $\mathrm{CO}_{2}^{+}$ion. Increasing quanta in the vibrations therefore lead to decreasing kinetic energies of the photoelectron. Due to the quantization, these show up as different rings in the photoelectron image in figure 3.12.

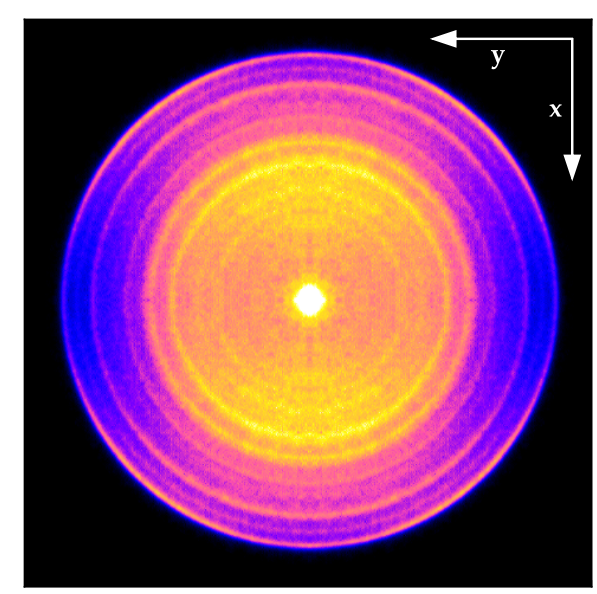

(a) crushed photoelectron image

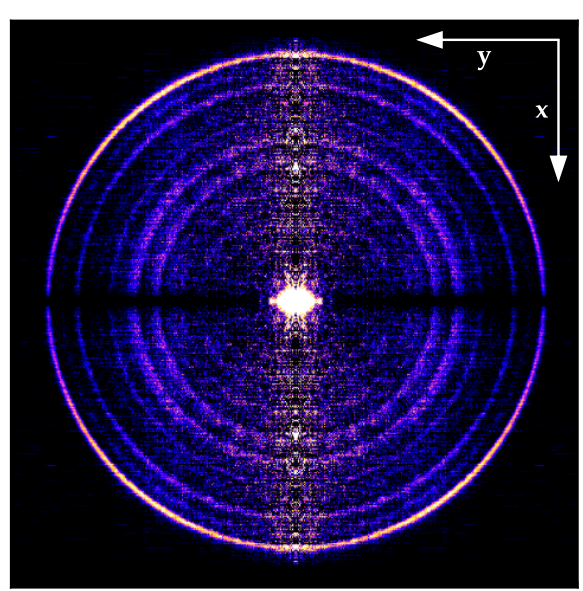

(b) slice image

Figure 3.12: Photoelectron image of $\mathrm{CO}_{2}$ obtained by $3+1$ REMPI via the intermediary $3 p \sigma_{\mathfrak{u}}{ }^{1} \Pi_{u}$ state. The laser polarization was aligned along $\mathrm{x}$.

Applying the inverse Abel transform to the crushed image in panel (a) yields the slice image in panel (b). The transform includes the intensity correction at each pixel by $r \sin (\vartheta)$ to reproduce the $3 \mathrm{D}$ intensities. Note that the algorithm used here introduces significant noise along the $x$-axis through the center. Integrating a sector of the slice image yields the vibrational state distribution of the excited state molecule, as reported in reference [26]. 


\subsubsection{Photofragmentation of nitrous oxide}

Another system investigated at the VMI machine was the photodissociation of $\mathrm{N}_{2} \mathrm{O}$. A dye laser (Sirah Precision Scan) with third harmonic generation unit produced 1-10 $\mathrm{mJ}$ of light at $200-215 \mathrm{~nm}$. This laser pulse was used both for initiation of the non-resonant singlephoton photodissociation of $\mathrm{N}_{2} \mathrm{O}$ as

$$
\mathrm{N}_{2} \mathrm{O}\left(X^{1} A^{\prime}\right)+h v \rightarrow \mathrm{N}_{2}\left(X^{1} \Sigma_{g}^{+}\right)+\mathrm{O}\left({ }^{1} \mathrm{D}\right)
$$

and for subsequent $2+1$ REMPI of the $\mathrm{N}_{2}$ fragment using the $\mathrm{a}^{\prime \prime}{ }^{1} \Sigma_{\mathrm{g}}^{+} \leftarrow$ $X^{1} \Sigma_{g}^{+}$transition $[27,28]$ around $203.5 \mathrm{~nm}$. Scanning the laser wavelength allowed us to selectively ionize $\mathrm{N}_{2}$ in different J-states of the

The small letter $a$ is used for historical reasons. vibrational ground state. An schematic showing the photodissociation process based on potential energy surface (PES) calculations by Schinke et al. [29] is shown in figure 3.13. A few vibrational levels are included for illustrative purposes.

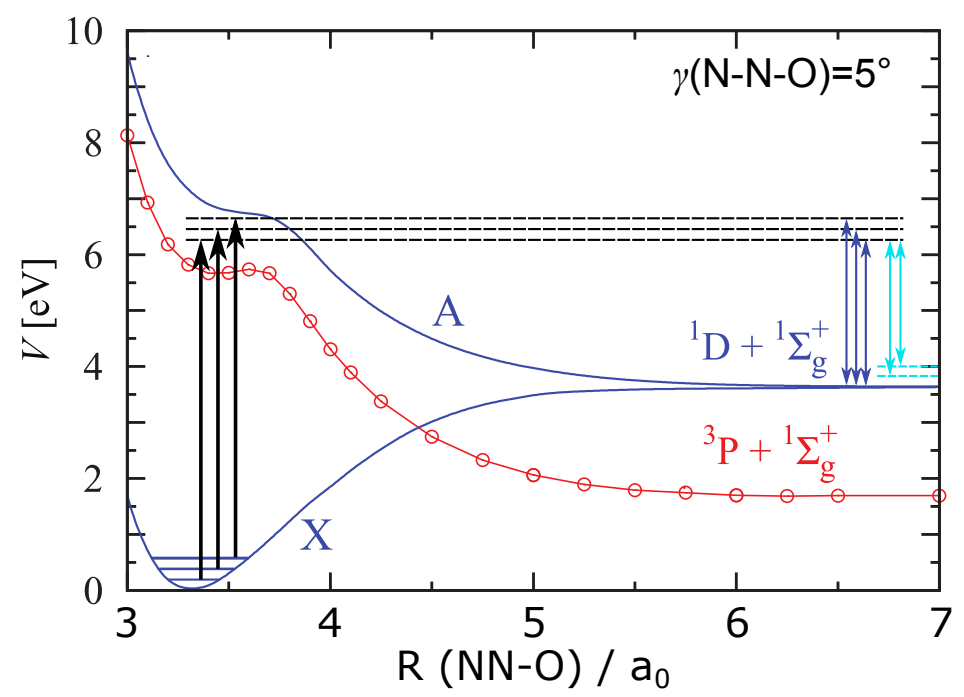

Figure 3.13: Schematic illustration of the $\mathrm{N}_{2} \mathrm{O}$ photodissociation: $\mathrm{N}_{2} \mathrm{O}$ in the electronic ground state $\mathrm{X}$ is excited to the predissociative state $\mathrm{A}$ by a single photon. It dissociates into $\mathrm{O}\left({ }^{1} \mathrm{D}\right)$ and $\mathrm{N}_{2}\left(X^{1} \Sigma_{g}^{+}\right)$. For details see text. Adapted from [29].

The kinetic energy release during the photodissociation depends on both the internal energy of the $\mathrm{N}_{2} \mathrm{O}$ molecule and the $\mathrm{N}_{2}$ fragment. Any internal energy of the electronic ground state of $\mathrm{N}_{2} \mathrm{O}$ (blue levels, curve $\mathrm{X}$ ) increases the energy of the dissociative excited state (dashed black lines). The different transitions are indicated by the black arrows. The energy of the excited state then partitions into the product internal energy, i.e. vibrational and rotational states of $\mathrm{N}_{2}$, and the kinetic energy of the products (blue arrows). The $\mathrm{O}\left({ }^{1} \mathrm{D}\right)$-atom is always in the same state and has no internal degrees of freedom. The $\mathrm{N}_{2}$ fragment thus contains all information on the internal energy of the parent molecule. If $\mathrm{N}_{2}$ is vibrationally or rotationally excited 
(cyan levels) this energy is deducted from the kinetic energy of the fragments (cyan arrows). In summary, the kinetic energy is decreased by vibrational or rotational energy of $\mathrm{N}_{2}$, while it is increased by vibrational energy of $\mathrm{N}_{2} \mathrm{O}$.

According to Schinke et al. $[29,30]$ the transition between the electronic ground state and the excited electronic state is dipole forbidden for linear $\mathrm{N}_{2} \mathrm{O}$ with $\mathrm{C}_{\infty v}$ geometry. The vibrational and electronic ground state in $\mathrm{C}_{\infty v}$ is ${ }^{1} \Sigma^{+}$and the excited states in our wavelength regions are ${ }^{1} \Sigma^{-}$and ${ }^{1} \Delta[31]$. The transition becomes allowed in the $\mathrm{C}_{\mathrm{s}}$ geometry, i. e. when bending excitations are populated in the electronic ground state. Note that the PES in figure 3.13 are calculated for a N-N-O bond angle of $5^{\circ}$. A larger bending angle also decreases the potential energy of the excited state A, which results in better overlap at our transition wavelength.

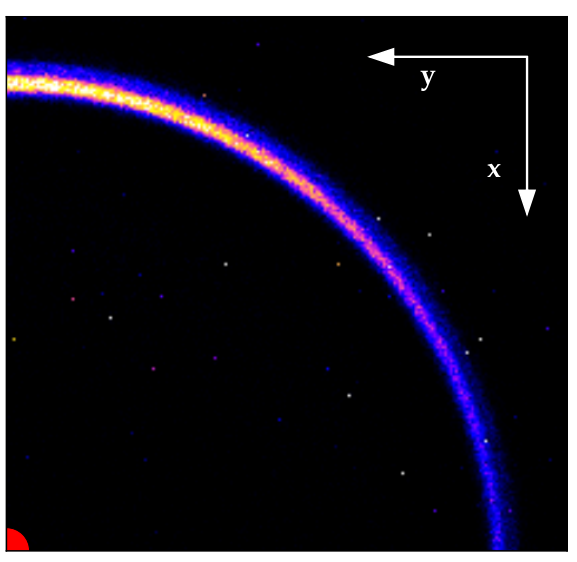

(a) Ion image of $\mathrm{N}_{2}(\mathrm{~J}=78)$
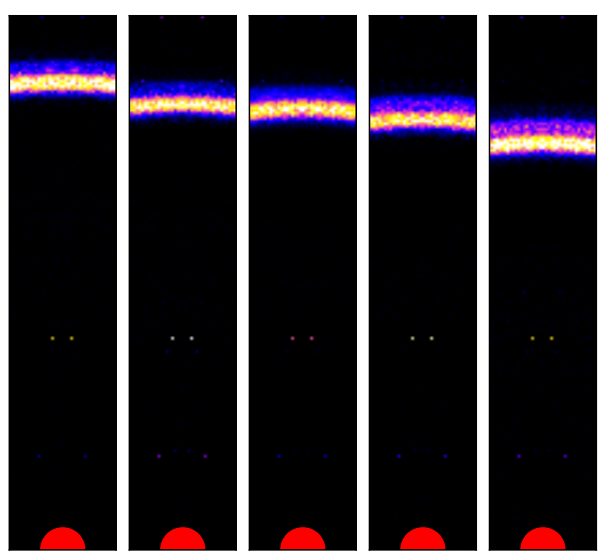

(b) $\mathrm{N}_{2}$ in $\mathrm{J}=70,73,74,75,78$

Figure 3.14: Ion images of $\mathrm{N}_{2}(\mathrm{v}=0)$ using $2+1$ REMPI at $\sim 203.2 \mathrm{~nm}$, generated from photofragmentation of $\mathrm{N}_{2} \mathrm{O}$. Panel (b) shows the kinetic energy release dependence on the rotational energy of the $\mathrm{N}_{2}$ fragment. The laser polarization was aligned along $\mathrm{x}$. The center of the ion image is indicated by the red dot.

Figure 3.14 shows an ion image of $\mathrm{N}_{2}(\mathrm{v}=\mathrm{O}, \mathrm{J}=78)$ in panel (a) and the different fragment velocities as a function of the $\mathrm{N}_{2}$ rotational

The wavelength was tuned to selectively ionize different J-states. state in panel (b). The $\mathrm{N}_{2}$ fragment in the $\mathrm{J}=70$ rotational state on the left has the largest velocity in this series. The fragment velocity decreases with higher rotational excitation.

By further analyzing the kinetic energy release of a single rotational state, e.g. the ion image at $\mathrm{J}=78$ shown in panel (a), we can infer the internal energy distribution of the $\mathrm{N}_{2} \mathrm{O}$ molecule prior to fragmentation. This is demonstrated by comparing the vibrational distribution of a beam of $\mathrm{N}_{2} \mathrm{O}$ expanded from a nozzle at room temperature to a beam expanded through a hot $\mathrm{SiC}$ tube. Quadrants of the ion images of the cold and the hot nozzle are shown in figure 3.15 (a) and (b). The image from the cold nozzle is dominated by a single ring. A second, 


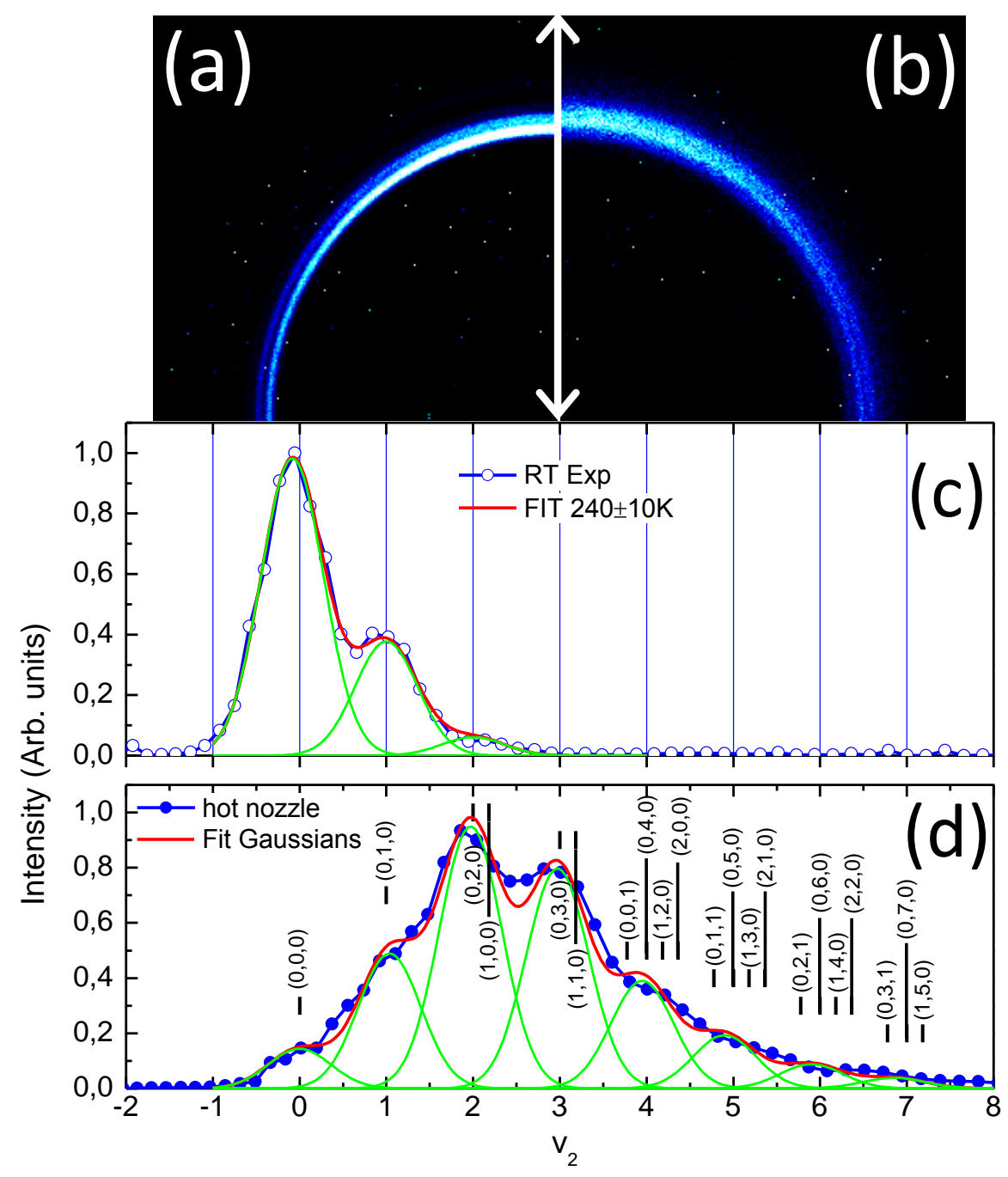

Figure 3.15: Ion images of $\mathrm{N}_{2}(\mathrm{v}=\mathrm{O}, \mathrm{J}=78)$ from photolysis of a hot nozzle $\mathrm{N}_{2} \mathrm{O}$ beam (b) compared to an expansion at room temperature (a). The vibrational population of $\mathrm{N}_{2} \mathrm{O}$ is obtained by integrating along a sector along the radius and shown in (c) for the cold and (d) for the hot nozzle. The signal in panel (c) and (d) is plotted as a function of kinetic energy. The kinetic energy labels are not shown. The kinetic energies corresponding to a discrete vibrational bending quanta $v_{2}$ are labeled. A series of Gaussians is fitted to deduce the vibrational temperature. Reproduced with permission from Ref. [24], (c) 2018 AIP. 
In other words the width of the ring is small compared to the radius. weaker ring at slightly larger velocities is visible. The image from the hot nozzle shows several rings at higher velocities. A higher velocity is a result of a higher $\mathrm{N}_{2} \mathrm{O}$ internal energy, i. e. a higher vibrational excitation.

We can thus extract the vibrational distribution by integrating along a sector, as shown in panels (c) and (d) as a function of the bend vibration $v_{2}$. The distribution in panel (d) was used to calculate photoabsorption cross sections for $0 \leqslant v_{2} \leqslant 7$. The analysis assumed an initially thermal population of all vibrations and efficient conversion of the quanta of stretch vibration into the degenerate bend vibration due to near-resonant V-V vibrational energy transfer [32] during the expansion. The full analysis has been reported in reference [24].

\subsubsection{Velocity resolution}

As can be estimated from the ion images in figures 3.14 and 3.15 the change in fragment velocity due to internal energy of the $\mathrm{N}_{2} \mathrm{O}$ prior to fragmentation is very small compared to the total velocity. The imaging setup thus needs a high velocity resolution to resolve the internal state distribution of the parent $\mathrm{N}_{2} \mathrm{O}$ molecule.
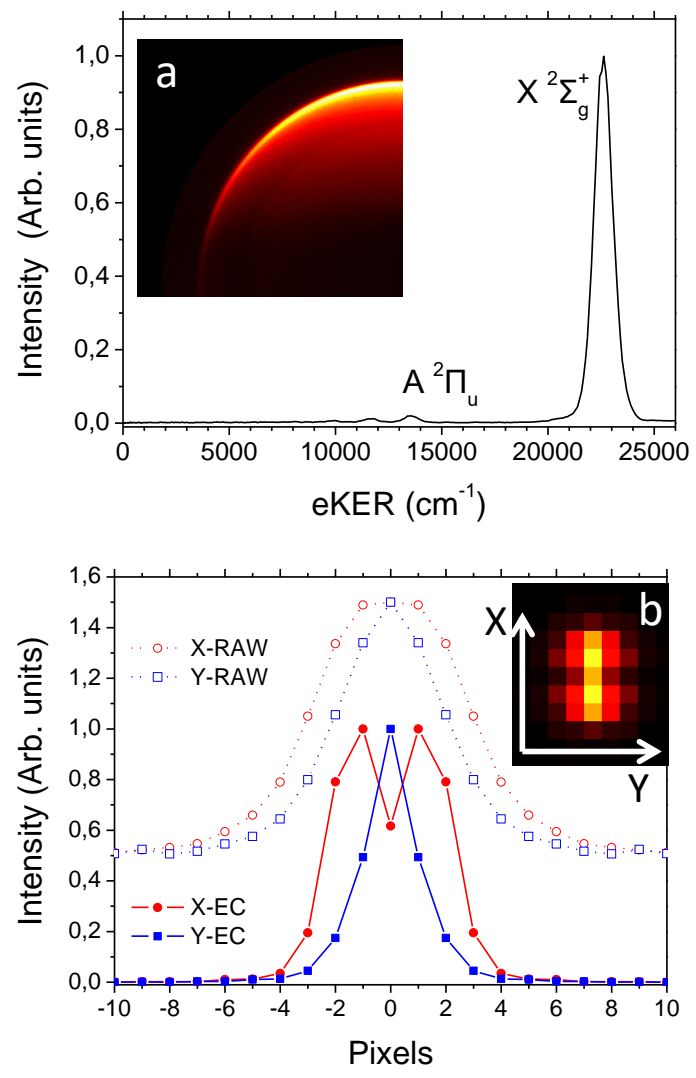

Figure 3.16: Demonstration of VMI resolution using $\mathrm{N}_{2}$ imaging: The top panel shows a VMI photoelectron image from $2+1$ REMPI of an $\mathrm{N}_{2}$ beam and the corresponding kinetic energy release spectrum. The lower panel shows an ion image of $\mathrm{N}_{2}^{+}$ obtained by combining VMI with event counting. The graph shows the intensity distribution along $\mathrm{x}$ and $\mathrm{y}$ for two ion images obtained with and without event counting. Reproduced with permission from Ref. [24], () 2018 AIP.

The resolution of the velocity setup presented here was demonstrated by imaging the electron recoil of the $\mathrm{N}_{2}^{+}$ion generated by $2+1$ REMPI of an $\mathrm{N}_{2}$ molecular beam. The upper panel in figure 3.16 
shows the kinetic energy release of the photoelectron. From an electron kinetic energy of about $22500 \mathrm{~cm}^{-1}$ and conservation of momentum we can calculate the $\mathrm{N}_{2}^{+}$ion kinetic energy to be $0.44 \mathrm{~cm}^{-1}$ and the velocity to be $20 \mathrm{~m} \mathrm{~s}^{-1}$.

The lower panel shows the result of further improving the $\mathrm{N}_{2}^{+} \mathrm{VMI}$ resolution by applying event counting. For ion images containing very few non-overlapping ion spots the center of each spot can be determined by fitting Gaussians along $x$ and $y$. A unit intensity at the center is then added to the event counting image. The profiles in the lower panel show histograms along $\mathrm{x}$ and $\mathrm{y}$ of the accumulated ion image (dotted lines) and the event counting image (solid lines). The laser was polarized along $x$. In the event counting image (small inset) and the intensity profile along $x$ (red solid line) shown in figure 3.16 the separation of the $\mathrm{N}_{2}^{+}$ions due to electron recoil is resolved. The separation of about 2-4 pixels corresponds to a velocity difference of $40 \mathrm{~m} \mathrm{~s}^{-1}$. Compared to velocities of about $2200 \mathrm{~m} \mathrm{~s}^{-1}$ in the photodissociation of $\mathrm{N}_{2} \mathrm{O}$ the resolution with event counting is on the order of $1-2 \%$.

\section{$3 \cdot 3$ CONCLUSIONS}

In this chapter I introduced imaging techniques and their applications to the study of gas phase processes. While spatial imaging is straightforward, the resolution can be greatly enhanced using velocity-map imaging - especially when combined with event counting. Both techniques can further be improved by slicing the ion cloud - this removes the need for an inverse Abel transform. A more comprehensive introduction to the various imaging techniques, presented by their respective inventors, can be found in B. Whitaker's Imaging in Molecular Dynamics [22]. Lastly, I have presented examples of several gas-phase imaging techniques, i. e. the result of the inverse Abel transform, a VMI investigation of a photodissociation process and the resolution available when using event counting.

\section{REFERENCES}

[17] David W. Chandler and Paul L. Houston. "Two-dimensional imaging of state-selected photodissociation products detected by multiphoton ionization." In: The Journal of Chemical Physics 87.2 (1987), p. 1445. DOI: 10.1063/1. 453276 (cit. on p. 19).

[18] David H. Parker and André T. J. B. Eppink. "Photoelectron and photofragment velocity map imaging of state-selected molecular oxygen dissociation/ionization dynamics." In: The Journal of Chemical Physics 107.7 (1997), p. 2357. Dor: 10 . 1063/1. 474624 (cit. on pp. 19, 21). 
[19] Christoph R. Gebhardt, T. Peter Rakitzis, Peter C. Samartzis, Vlassis Ladopoulos, and Theofanis N. Kitsopoulos. "Slice imaging: A new approach to ion imaging and velocity mapping." In: Review of Scientific Instruments 72.10 (Oct. 2001), pp. 38483853. DOI: 10.1063/1. 1403010 (cit. on pp. 19, 26).

[20] D. J. De Rosier and A. Klug. "Reconstruction of three dimensional structures from electron micrographs." In: Nature 217.5124 (Jan. 1968), pp. 130-134. DOI: 10.1038/217130a0 (cit. on p. 19).

[21] M. Zwick and E. Zeitler. "Image Reconstruction from Projections." In: Optik 38 (1973), pp. 550-565 (cit. on p. 19).

[22] B. Whitaker. Imaging in Molecular Dynamics. Ed. by Benjamin J. Whitaker. Cambridge: Cambridge University Press, 2003. DoI: 10. 1017/CB09780511535437 (cit. on pp. 21, 26, 35).

[23] Nils Henrik Abel. "Auflösung einer mechanischen Aufgabe." In: Journal für die reine und angewandte Mathematik 1 (1826), pp. 153-157 (cit. on p. 22).

[24] Dan J. Harding, Jannis Neugebohren, M. Grütter, A. F. SchmidtMay, D. J. Auerbach, T. N. Kitsopoulos, and A. M. Wodtke. "Single-field slice-imaging with a movable repeller: Photodissociation of $\mathrm{N2}$ O from a hot nozzle." In: The Journal of Chemical Physics 141.5 (Aug. 2014), p. 054201. DOI: 10.1063/1. 4891469 (cit. on pp. 26, 29, 33, 34).

[25] Daniel W. Kohn, Horst Clauberg, and Peter Chen. "Flash pyrolysis nozzle for generation of radicals in a supersonic jet expansion." In: Review of Scientific Instruments 63.8 (1992), pp. 40034005. DOI: 10.1063/1.1143254 (cit. on p. 30).

[26] Ming Wu, David P. Taylor, and Philip M. Johnson. "Resonance enhanced multiphoton ionization photoelectron spectra of $\mathrm{CO}_{2}$. II. Competition between photoionization and dissociation." In: The Journal of Chemical Physics 95.2 (1991), p. 761. Dor: 10.1063/ 1.461082 (cit. on p. 30).

[27] Barry L. Lutz. "Presscure-Induced a" ${ }^{1} \Sigma_{g}^{+} \leftarrow X^{1} \Sigma_{g}^{+}$Absorption in the Vacuum Ultraviolet Spectrum of Molecular Nitrogen." In: The Journal of Chemical Physics 51.2 (1969), pp. 706716. DOI: 10.1063/1.1672060 (cit. on p. 31).

[28] Keith R. Lykke and Bruce D. Kay. "Two-photon spectroscopy of $\mathrm{N}_{2}$ : Multiphoton ionization, laser-induced fluorescence, and direct absorption via the a' ${ }^{1} \Sigma_{\mathrm{g}}^{+}$state." In: The Journal of Chemical Physics 95.4 (Aug. 1991), pp. 2252-2258. DOI: 10.1063/1. 460981 (cit. on p. 31).

[29] R. Schinke, J. A. Schmidt, and M. S. Johnson. "Photodissociation of $\mathrm{N}_{2} \mathrm{O}$ : Triplet states and triplet channel." In: Journal of Chemical Physics 135.19 (2011). DoI: 10.1063/1. 3660349 (cit. on pp. 31, 32). 
[30] R. Schinke. "Photodissociation of $\mathrm{N}_{2} \mathrm{O}$ : Potential energy surfaces and absorption spectrum." In: Journal of Chemical Physics 134.6 (Feb. 2011), p. 064313. DOI: 10.1063/1. 3553377 (cit. on p. 32).

[31] Darrel G. Hopper. " $A b$ initio multiple root optimization MCSCF study of the $\mathrm{C}_{\infty v} / \mathrm{C}_{s}$ excitation spectra and potential energy surfaces of $\mathrm{N}_{2} \mathrm{O}$." In: The Journal of Chemical Physics 80.9 (May 1984), pp. 4290-4316. DoI: 10. 1063/1. 447260 (cit. on p. 32).

[32] J. D. Lambert. Vibrational and rotational relaxation in gases. Oxford: Clarendon Press, 1977, p. 141 (cit. on p. 34). 



\subsubsection{Challenges and limitations in previous experiments}

Compared to the imaging of gas-phase processes, the imaging of processes at surfaces is experimentally much more challenging. The experiments described in the earlier chapter all rely on well-defined, symmetric electric fields. Any distortion to these fields must be eliminated. A metal surface at ground level, however, easily causes such a distortion.

The earliest imaging experiment on processes at surfaces by Niehus et al. [33] from 1981 predates even the seminal imaging paper by Chandler and Houston. In this experiment a channeltron (channel electron multiplier) on a goniometer was used to measure an electron stimulated desorption ion angular distribution (ESDIAD) of $\mathrm{O}^{+}$desorbing from $\mathrm{Mo}(111)$. In a second configuration the goniometer could be replaced by an $\mathrm{MCP} /$ phosphor stack behind three hemispherical LEED grids, as shown in figure 4.I. The author then compares the

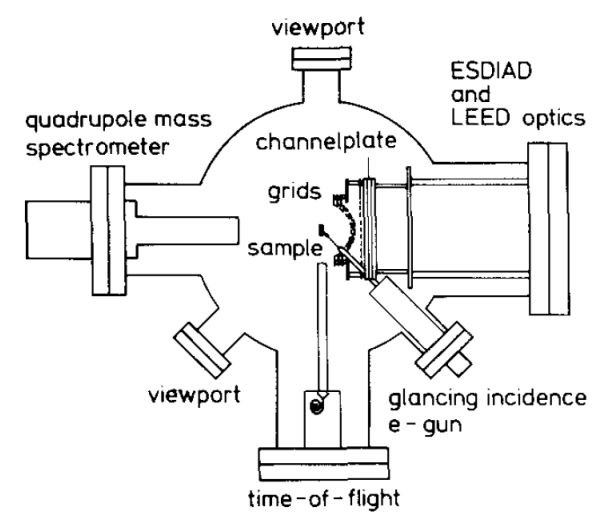

Figure 4.1: First imaging of processes at surfaces setup. Reproduced from [33].

imaging technique to the goniometer technique. Since the imaging plane is parallel to the surface, the movement component of desorbing ions parallel to the surface is detected on the phosphor screen. This setup allows the angular distributions to be recorded but does not provide any information on the speed of the desorbing ion.

Another application by Kobrin et al. [34] was presented in 1986. The experimental setup is shown in figure 4.2. In this experiment the surface is tilted $45^{\circ}$ to the imaging plane. A pulsed normal-incident ion 
beam impinging on a polycrystalline rhodium sample leads to desorbtion of neutral rhodium atoms with $0-20 \mathrm{eV}$ kinetic energy. After passing the grid $G_{1}$, the rhodium atoms are ionized using a REMPI laser, which acts as a time of flight selector. The ions are then accelerated towards an $\mathrm{MCP} /$ phosphor detector, labeled $\mathrm{M}_{1,2,3} / \mathrm{P}$. The posi-

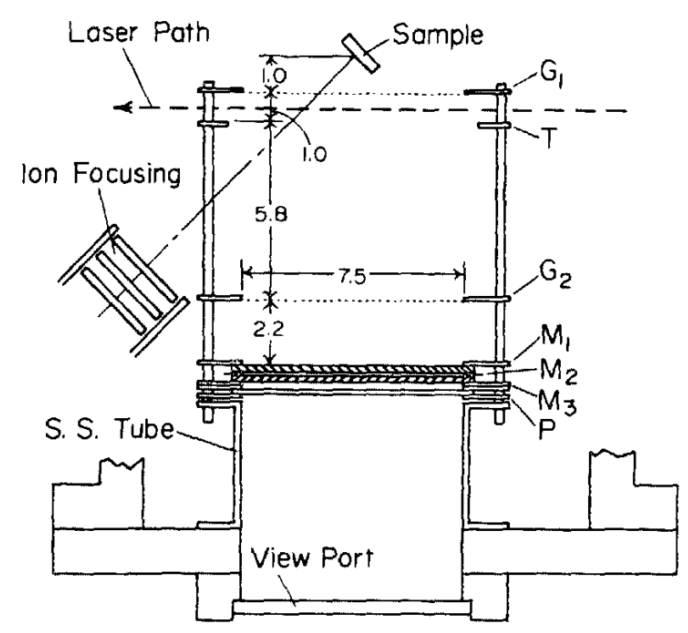

Figure 4.2: Imaging of processes at surfaces setup by Kobrin et al. Reproduced from [33].

tion on the detector is a combination of desorption angle and speed. After substantial corrections a deconvolution allows these quantities to be extracted.

While this technique allows the reconstruction of both angular and speed distribution, the applicability to measurements of trappingdesorption or reactions at surfaces is limited. Due to geometrical restrictions the ion beam initiating the desorption passes through the grids belonging to the ion optics. In a trapping-desorption experiment, a large fraction of the incident molecular beam would collide with the grids of the ion optics labeled $G_{1}$ - one would not be able to distinguish between desorption from the grid and desorption from the surface.

\subsubsection{Our experimental configuration}

In contrast to the two experiments above, the imaging plane at Beamer II is perpendicular to the surface plane. This allows both the speed and the angular distribution to be recorded simultaneously in each ion image.

The ion optics consist of two parallel grids, a repeller grid and a grounded grid, and an open ring electrode (Einzel lens) as shown at the bottom in figure 4.3. The two grids are used to generate a homogeneous electric field and to accelerate ions or electrons towards the detector. Both grids are made of 670 lines-per-inch (LPI) nickel 


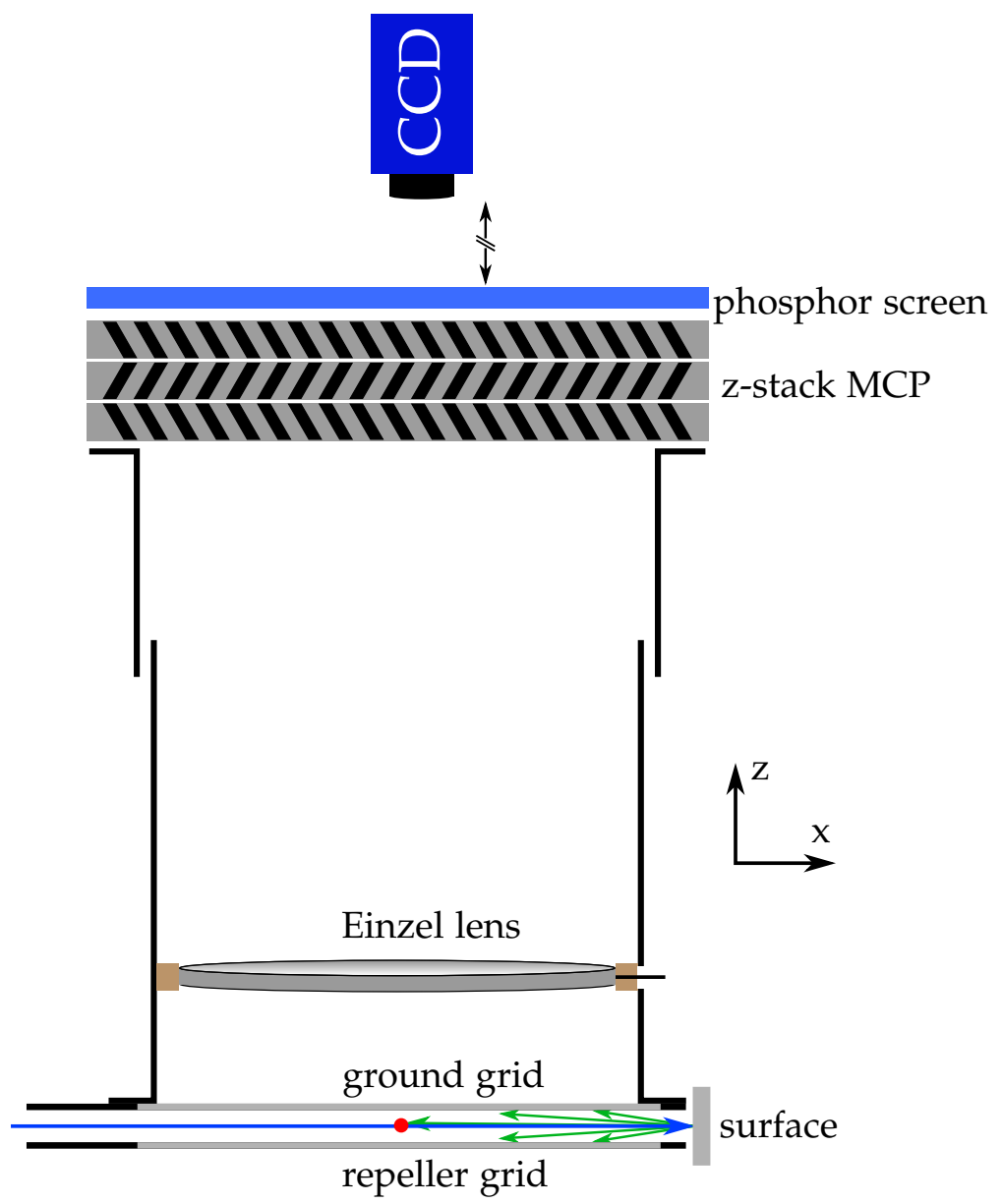

Figure 4.3: Schematic of the imaging unit arrangement. An incoming beam is shown as a blue arrow, scattered molecules as green arrows and the laser focus as a red circle.

mesh (Precision Eforming, 49\% transmission) glued to a stainlesssteel frame by graphite adhesive.

The distance between the grids is large enough to allow safe passage of the laser, but as small as possible to reduce stray fields. A homogeneous field is critical to ensure that only a vertical velocity component is applied to ions in the extraction region. Stray fields not only influence the homogeneity, but also severely distort the ion image when delayed extraction is used. In this technique the molecules continue to move along their initial trajectory after ionization before the extracting field is pulsed. A stray field on the order of $1 \mathrm{~V} \mathrm{~cm}^{-1}$ would be enough to have a large effect on this movement compared to typical kinetic energies in the sub-eV range. The penetration depth of distortions depends on the distance between the repeller and the ground grid. A value of $5 \mathrm{~mm}$ turned out to be a good compromise.

The open-ring Einzel lens above the extractor grid allowed the imaging mode to be converted from spatial imaging to velocity-map

The principle of delayed extraction has been introduced in section 3.1.1.

Proper VMI voltage calibration is explained in section 3.1 .2 
phosphor

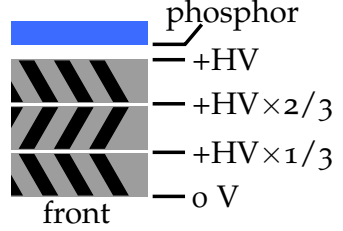

imaging by floating the lens at a certain voltage. For spatial imaging the Einzel lens was grounded.

The three z-stacked MCPs and the phosphor screen in the upper part of figure 4.3 comprise the detection unit. The MCPs were rotated approximately $120^{\circ}$ against each other to minimize the resulting offset of the electron signal. In continuous mode a voltage between 1500 and $2100 \mathrm{~V}$ was evenly divided across the 3 layers, with the front of the first MCP being at ground and the back of the third MCP at the full positive voltage. Higher signal amplification can be achieved if the front is floated at a negative voltage, but the flight path of the ions would be distorted and this setup is therefore unsuitable for imaging.

The MCP stack could also be operated in pulsed mode: Here the continously applied gain across the MCPs is reduced to e.g. $1400 \mathrm{~V}$ and a fast-rising ( $<20 \mathrm{~ns})$ voltage pulse of e.g. $-800 \mathrm{~V}$ is applied to the front of the first MCP for 80-30o ns. The delay between MCP gate and the extraction pulse acts as a mass discriminator and the width of the voltage pulse acts as acceptance range. Only ions with a remaining time-of-flight (TOF) smaller than the gate duration will be amplified. In other words only ions which are very close to the MCP already will be detected. The flight path distortion of said ions due to the electric field is negligible. The low duty cycle of the MCP greatly decreases depletion effects due to background ions or light and must therefore be utilized when the filament is used to heat the surface.

The phosphor screen on top of the third MCP was separated by a $2 \mathrm{~mm}$ gap. Applying a positive voltage of $3000-4000 \mathrm{~V}$ cause the electron cloud generated by the MCP cascade to cross the vacuum gap and light up the phosphor screen. When applying voltages greater than $3000 \mathrm{~V}$ the phosphor screen should be pulsed to avoid accidental discharges. The phosphor layer (ProxiVision $\mathrm{P}_{43}, 90 \%$ decay in $1 \mathrm{~ms}$, maximum emission at $545 \mathrm{~nm}$ ) was grown on a quartz substrate covered by a conductive ITO layer. The images on the phosphor screen are recorded with a LaVision Imager E-lite CCD camera and custommodified Davis software.

The molecular beam (blue arrow) in figure 4.3 passes between two grids and impinges on the metal crystal. The molecules are scattered at different angles between the repeller and the grid (green arrows). Only molecules with very little velocity along the $\mathrm{z}$ axis reach the laser focus while the other molecules collide with the grids. The distance between laser focus and the metal crystal is chosen to be large enough to avoid the distortions of the electric field by the metal surface. 


\subsubsection{Ion image of $\mathrm{N}_{2}$ scattering - an example}

An ion image of $\mathrm{N}_{2}$ scattered from $\mathrm{Au}(111)$ is shown in figure 4.4 and shall be used to illustrate the typical features. $\mathrm{N}_{2}$ is ionized state-

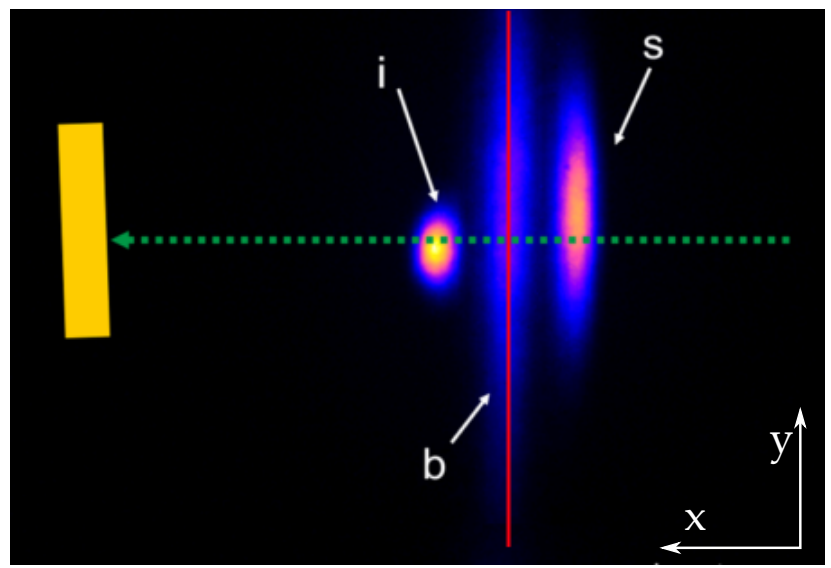

Figure 4.4: The ion image shows three features: Molecules from the incident beam (i) moving towards the surface, molecules scattered from the surface moving to the right (s) and background $\mathrm{N}_{2}$ ions (b) symmetric around the laser (red line). Reprinted with permission from Ref. [35], (c) 2018 ACS.

selectively at the laser position indicated by the red line using $2+1$ REMPI at $203 \mathrm{~nm}$ through the $\mathrm{a}^{\prime \prime}{ }^{1} \Sigma_{\mathrm{g}}^{+}$state. Since the pulsed electric field only accelerates along the z-axis, ions with zero velocity end directly above the laser path. Ions from background $\mathrm{N}_{2}$ in the chamber have a uniform velocity distribution and the corresponding ion signal, labeled " $\mathrm{b}$ ", is symmetric around the laser position. Ions with a velocity towards the surface end up to the left of the laser, as is the case for ions from the incident beam labeled " $\mathrm{i}$ ". Ions moving away from the surface, labeled " $\mathrm{s}$ " for scattering, end up on the right of the laser.

\subsection{DYNAMICAL PARAMETERS FROM ION IMAGES}

In this section I will introduce the dynamical parameters seen in imaging of processes at surfaces. First I give a brief review which part of the flux is detected in our experimental setup and how to describe it in the polar coordinate system. I will then explain the density-to-flux conversion and the extraction of angular and velocity distributions. Lastly, I will introduce the analytic equations characterizing the flux leaving a surface. 


\subsubsection{Coordinate system}

The coordinate system we use to describe processes at surfaces is similar to the one introduced in figures 3.4 and 3.6 in chapter 3. Instead of an isotropic distribution, the flux leaving the surface generates a hemisphere, as shown in figure 4.5 .

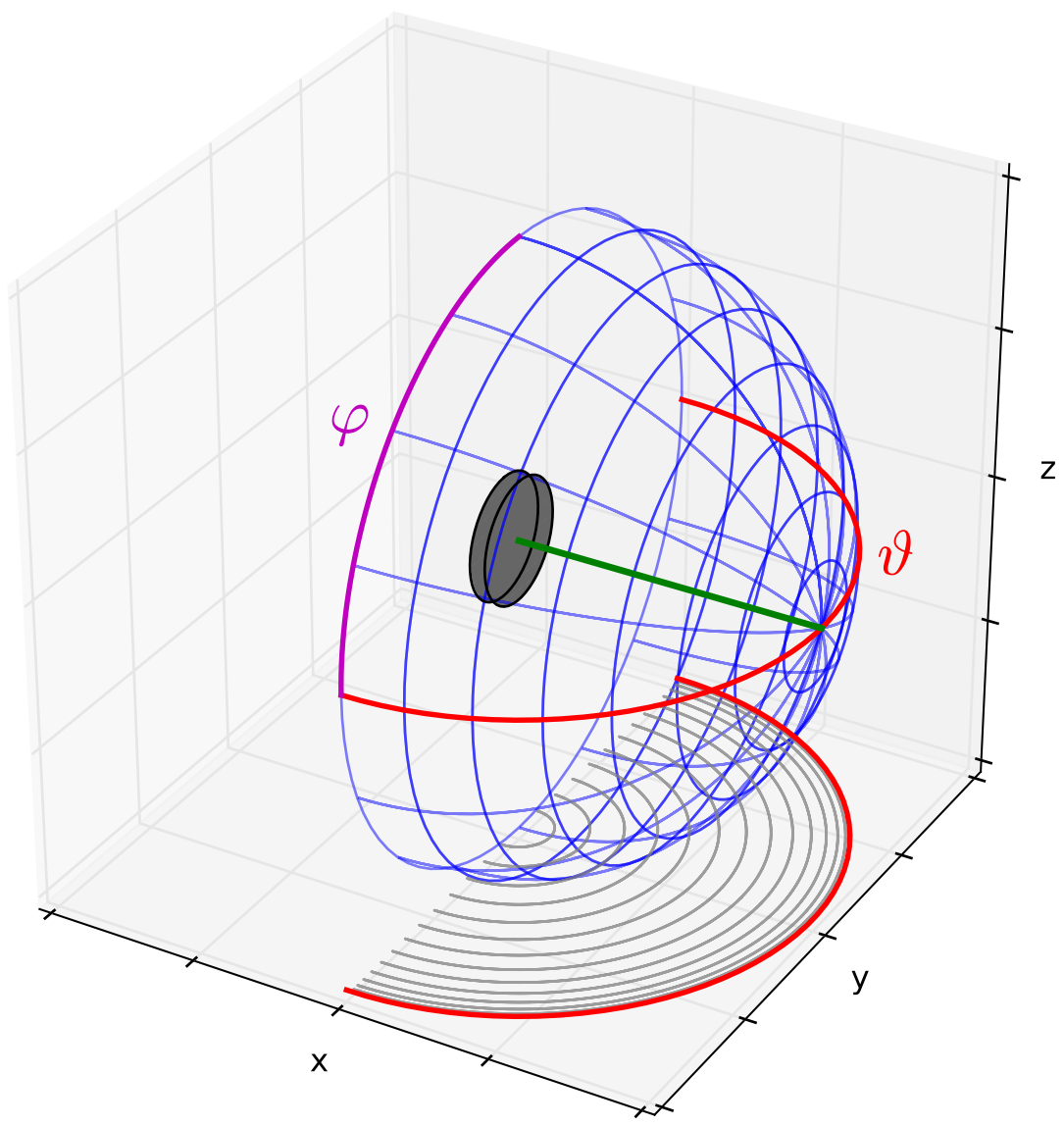

Figure 4.5: Imaging geometry of processes at surfaces.

The surface is plotted as a dark gray disc, with the surface normal as a green line. The flux leaving the surface is rotationally symmetric around the surface normal (cylindrical symmetry). If all molecules leaving the surface were detected, the resulting crushed image would show an intensity distribution as indicated by the gray lines in the $x y-$ plane. The laser, however, is parallel to the y-axis and slices the ion cloud in the xy-plane at a finite distance from the surface. Molecules with substantial velocities along $\mathrm{z}$ are not ionized - this is called laser slicing [36]. The molecules ionized by the laser are indicated by the red line. In the slice ion image these molecules appear as a semicircle, also indicated by a red line. The distribution of molecules along $\vartheta$ is preserved in the slice ion image. Under the assumption of cylindrical symmetry, one can reconstruct the $3 \mathrm{D}$ intensity by weighting each point in the slice image by $2 \pi r \sin (\vartheta)$, as introduced in section 3.1.3. 


\subsubsection{Angular distributions from ion images}

The apparent angular distribution in experiments at surfaces depends not only on the desorption process but also on the detector geometry. For experimental setups such as a quadrupole mass spectrometer (QMS) mounted on a goniometer the effect of the detector geometry on the observed signal has been carefully explained by Comsa et al. [37]. I now want to adapt this description to our experimental setup to discuss whether there is a need to correct for any geometrical factors.

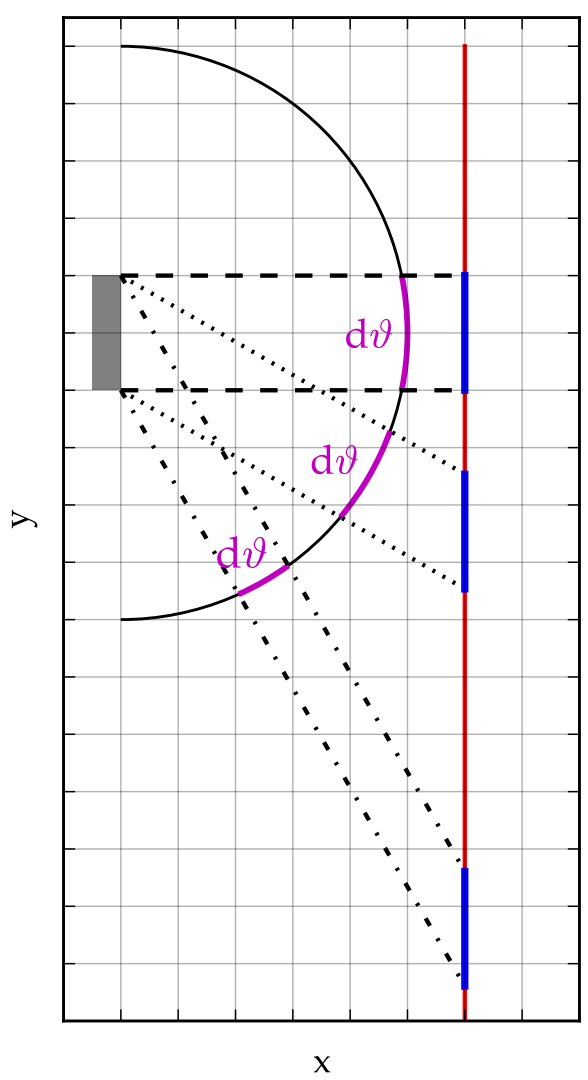

Figure 4.6: Detector geometry in angular distributions: The surface is shown as a grey rectangle. An isotropic desorption process is indicated by the black semicircle around the center of the surface and an ionizing laser is plotted as a red line. The desorption fluxes along the surface normal (dashed lines), along $30^{\circ}$ (dotted lines) and along $60^{\circ}$ (dash-dotted lines) occupy a different solid angle $d \vartheta$ at the semicircle (magenta line) but the same distance along the laser focus (blue line).

Consider a layer of molecules on a surface which desorb with an isotropic emission probability as shown in figure 4.6. The number of molecules desorbing along the surface normal $\left(\vartheta=0^{\circ}\right)$ between the two dashed lines is the same as the number desorbing along $\vartheta=30^{\circ}$ (dotted lines) or along $\vartheta=60^{\circ}$ (dot-dashed lines). The solid angle $d \vartheta$ encompassed by these lines decreases at larger angles, as indicated by the magenta lines. The same number of molecules desorb at higher angles but the molecules pass through a smaller solid angle d $\vartheta$. A non-collimated QMS detector with a finite inlet aperture which rotates along the black circle will thus capture a different flux depending on the angle. The measured flux would scale with $\cos ^{-1}(\vartheta)$. In goniometer experiments this geometrical factor can be avoided by using well collimated detectors with additional apertures. In our ex-

An isotropic (not cosine) desorption is unrealistic but simplifies this example. 
A dark image is the mean background per image, averaged over $\sim 30$ images. periment, however, the flux is ionized at the laser focus indicated by the red line. The length $\Delta y$ along the laser focus the ions occupy is indicated by the blue lines. Note that the length is the same as the surface diameter, and that it is independent of the angle. The density of molecules at the laser focus is independent of the angle. The ion image detector needs no collimation nor any geometrical factor to capture the proper angular distribution of the desorption process. The only aberation present in spatial imaging is the extent of the surface or the extent of the molecular beam impinging on it. The resolution of the apparatus can be estimated by comparing the surface diameter or the molecular beam diameter to the total length of the signal along y. Note that the extent of the source can be collapsed in VMI, which would greatly enhance this resolution.

The process of calculating an angular distribution from an ion image shall be described using the desorption of $\mathrm{CO}$ from $\mathrm{Pt}(111)$. $\mathrm{CO}$ desorbing from $\mathrm{Pt}(111)$ was ionized by $2+1$ REMPI at $230 \mathrm{~nm}$ using the $\mathrm{B}^{1} \Sigma^{+} \leftarrow \mathrm{X}^{1} \Sigma^{+}$transition. A recorded image is shown in figure 4.7a. Under similar conditions an ion image of uniformly distributed $\mathrm{CO}$ background gas was recorded, see figure $4.7 \mathrm{~b}$. A CO background pressure of $2 \times 10^{-7}$ mbar was generated by leaking pure $\mathrm{CO}$ into the chamber.

Before an angular distribution can be calculated, the ion image must be corrected for background intensity from e. g. stray light or stray ions. The background intensity is already decreased by subtracting a dark image from each image during recording. Any remaining intensity is now removed by subtracting the mean intensity in a chosen region of interest (ROI) far away from the ion spots. If the surface position is known, we then calculate the angle $\vartheta$ for each pixel as

$$
\vartheta=\arcsin \left(\frac{\Delta y}{\Delta x}\right) \cdot \frac{180}{\pi}
$$

where $\Delta x$ is the distance between the surface and the pixel along $x$ and $\Delta y$ the distance from the surface normal, indicated by the dotted green line in figure $4.7 \mathrm{~b}$, and the pixel along $\mathrm{y}$. After binning the resulting distribution over a chosen increment, e.g. 1 or $2^{\circ}$, a raw angular distribution is obtained.

Dividing the distribution of the ion signal from fig. $4.7 \mathrm{a}$ by the distribution obtained from the background gas in fig. $4.7 \mathrm{~b}$ yields the actual angular distribution shown in figure 4.8. This division corrects for both laser intensity along the focus direction and inhomogeneous detection efficiency of the MCP stack.

A fit $f(\vartheta)$ to the data in figure 4.8 with

$$
\begin{aligned}
f(\vartheta) & =a_{1} \cdot \cos \left(\vartheta-\beta_{1}\right)^{n_{1}}+a_{2} \cdot \cos \left(\vartheta-\beta_{2}\right)^{n_{2}} \\
& =0.20 \cdot \cos (\vartheta-0.66)^{0.92}+0.88 \cdot \cos (\vartheta-0.50)^{183}
\end{aligned}
$$

is shown as a blue line. As expected for this process $[37,38]$ the trapping desorption component shows a cosine distribution. Due to the 


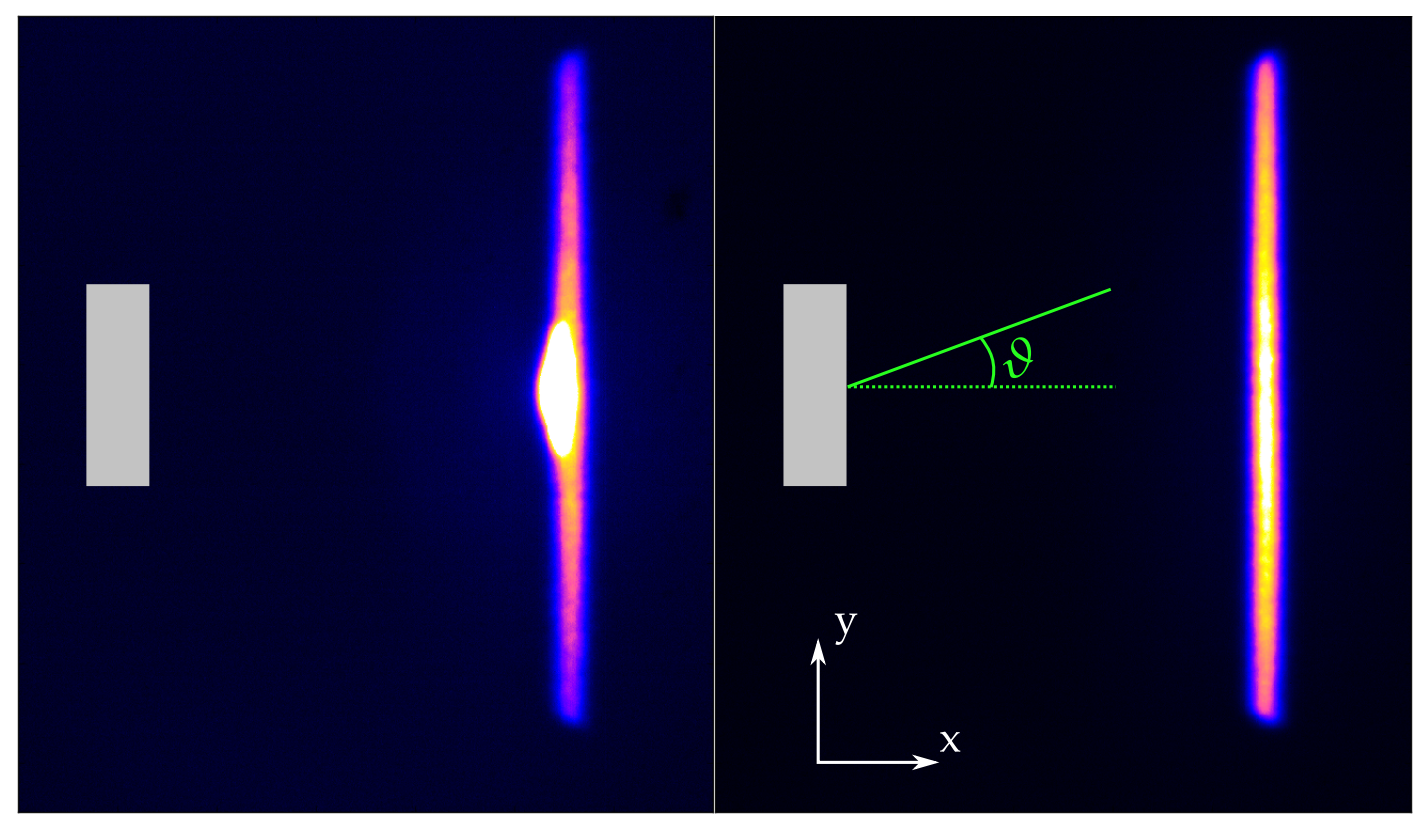

(a) Incident $\mathrm{CO}$ beam and desorbing $\mathrm{CO}$

(b) CO background gas

Figure 4.7: Angle-integrated ion images of $\mathrm{CO}$. (a) Incident $\mathrm{CO}$ beam in the middle and $\mathrm{CO}$ desorbing from $\mathrm{Pt}(111)$ around it. The ion image was taken at $\mathrm{T}_{\mathrm{S}}=753 \mathrm{~K}$. (b) $\mathrm{CO}$ ions generated from a uniformly distributed background gas. Any signal variation from top to bottom is due to the detector sensitivity and laser focus. The ionization probability changes with the focusing along the laser propagation.
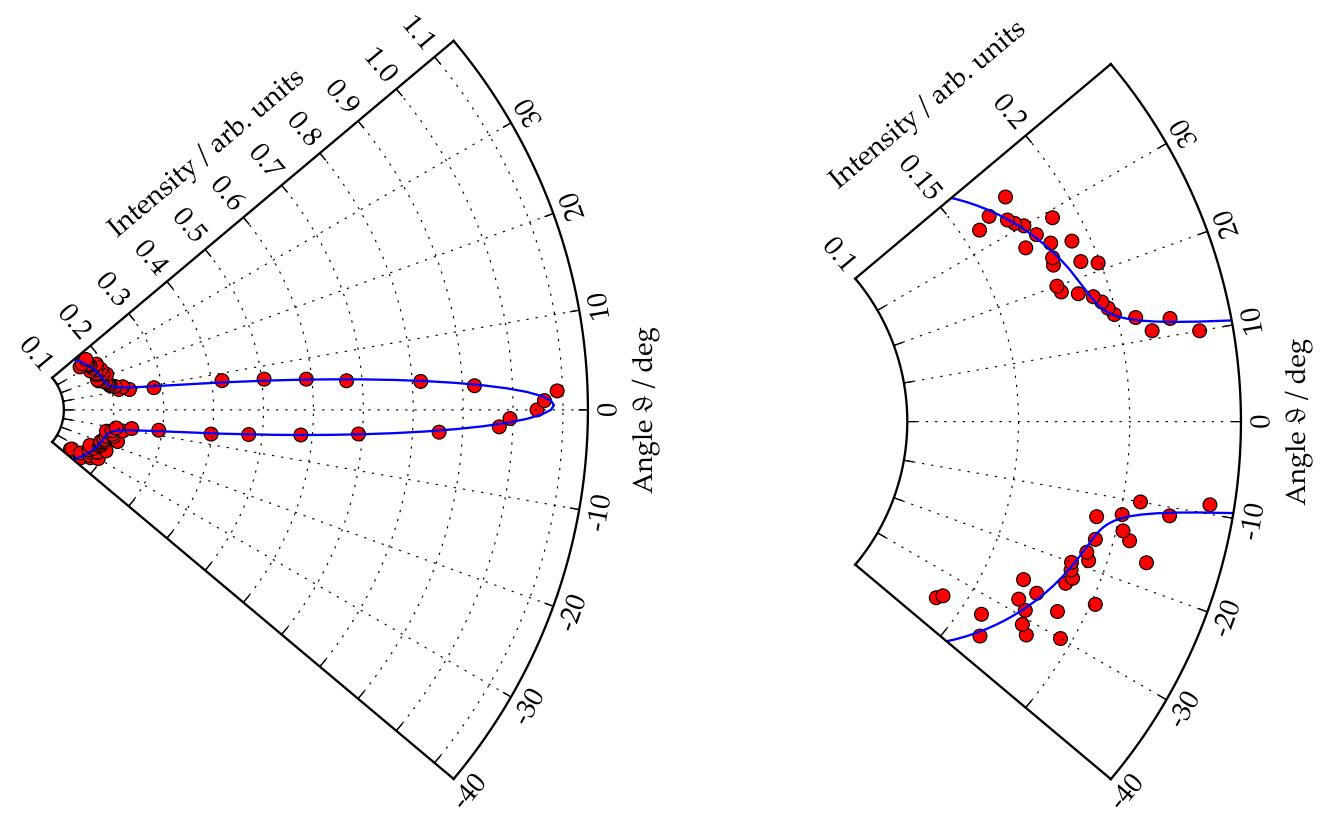

Figure 4.8: Angular distribution of $\mathrm{CO}$ desorbing from $\mathrm{Pt}(111)$ and the incident $\mathrm{CO}$ beam along the surface normal. The fit function $f(\vartheta)$, blue line, is described in the text. A zoom on the broad component is shown on the right. 
spatial extent of the beam on the surface, we would expect the angular distribution to be slightly broadened, which is the case here.

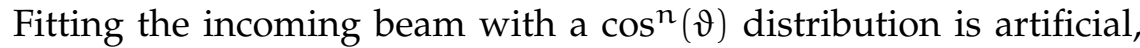
but works nonetheless. From a FWHM of $5^{\circ}$ and a surface-laser distance of about $17 \mathrm{~mm}$ we can estimate the molecular beam FWHM diameter to be $3 \mathrm{~mm}$.

\subsubsection{Density to flux in imaging}

The laser slicing in our experiment has ionization probabilities proportional to the density of molecules in the ionization volume. We are, however, interested in the flux of molecules leaving the surface. Our imaging setup has the great advantage to inherently record the ion velocities in the xy-plane. We can thus correct the ionization probability bias by multiplying the intensity at each pixel with the corresponding speed.

The speed per pixel is calculated as follows:

1. The ion is assumed to fly along a vector determined by the pixel and the middle of the surface.

2. Ions between laser and surface moved towards the surface, ions on the other side of the laser moved away from the surface.

3. The ion was generated at the intersection of the velocity vector and the laser.

4. The speed is proportional to the distance of the pixel from the intersection along the vector.

While a value proportional to the speed is sufficient for the densityto-flux conversion, the absolute speeds can be calculated as

$$
v=\frac{\Delta x}{24.5 \text { pixels } / \mathrm{mm} \times \mathrm{t}_{\mathrm{TOF}}},
$$

The calibration of pixel per $\mathrm{mm}$ is shown in the appendix in section C.1.

All vectors (black arrows) have the same length, i.e. the same speed. where $\Delta x$ is the distance in pixel and $t_{\mathrm{TOF}}$ is the time-of-flight.

This relation of pixel position and velocity is illustrated in the diagrams in figure 4.9. Panel (a) shows a surface with continuously desorbing molecules of the same velocity. Only semicircles are shown. The laser intersects different rings, i.e. the laser ionizes molecules with the same speed but different flight times to the laser. In panel (b) these ions continue to move along their initial direction before impinging on the detector. The resulting pattern seen on the detector (blue line) is no longer a semicircle. Panel (c) shows an example for such a velocity distribution. The image shows the signal from direct scattering and recombinative desorption of $\mathrm{H}_{2}$ on $\mathrm{Pt}(111)$. The ion spots in the lower half of the image show the same shape as predicted in panel (b). The bright (space-charged) ion spot in the upper part of the ion image is the tail of the incident $\mathrm{H}_{2}$ beam. 


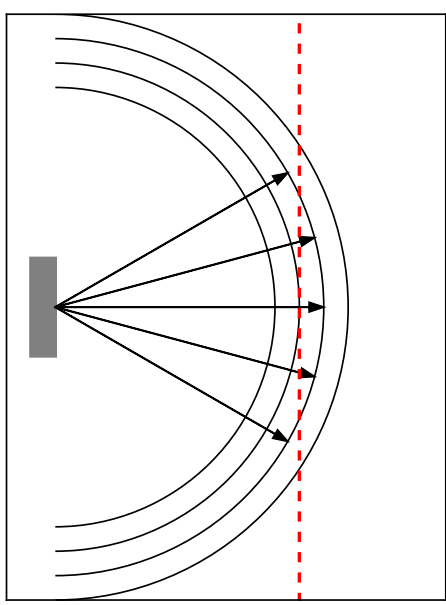

(a) Desorbing molecules

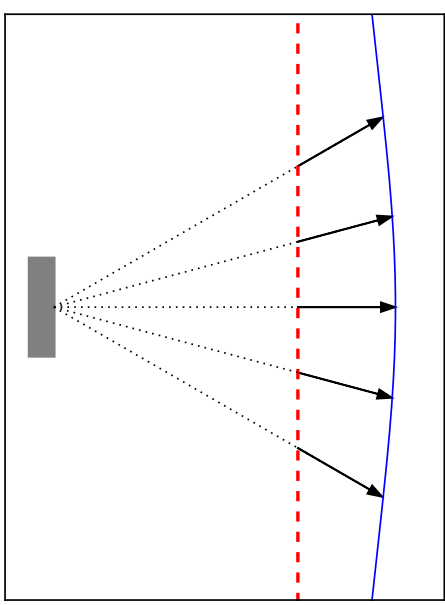

(b) Observed velocities

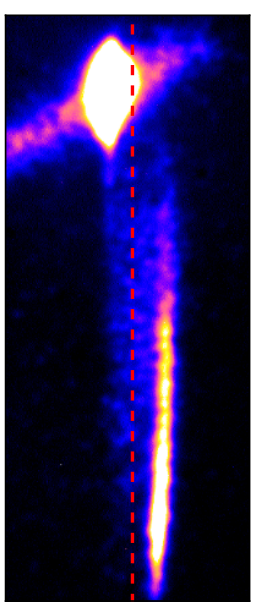

(c) Ion image

Figure 4.9: Observed velocities a) Molecules continuously desorb with the same speed. b) Molecules ionized by the laser travel along their initial direction with the same speed. The resulting position on the detector is shown as a blue line. c) Example ion image of $\mathrm{H}_{2}$ scattering/recombination, which shows the same curvature as the blue line.

Using the procedure as defined above, we can calculate the velocities for an array with the same dimensions as the ion image. A qualitative example array is shown in figure 4.10, note that the dimensions are chosen arbitrarily. The color gradient to the left and the right of the laser shows the same curvature as illustrated in the previous figure. The velocities were calculated assuming a flight time of $2 \mu \mathrm{s}$. Since we assume all ions to be generated at the laser position and move along a line through the surface center, pixels in the upper and lower left corners have unrealistically high velocities. As the angle $\vartheta$ approaches $90^{\circ}$ the velocity would go towards infinity. A cutoff function setting these velocities to zero is introduced to avoid distortions when multiplying the conversion array with the density image.

The conversion array as shown here can be used to convert both ion images of scattered molecules and ion images of the incident beam. The assumption that all ions move in straight lines towards or away from the surface center should be true in any case.

\subsubsection{Speed distributions in surface scattering experiments}

The speeds of ideal gas molecules in thermal equilibrium, i.e. normally distributed speeds along each of the three spatial axes, are described by the Maxwell-Boltzmann (MB) distribution. One form of the $\mathrm{MB}$ distribution describing the volume number density, i.e. the number of molecules with speeds between $v$ and $v+d v$ in a unit volume, is

The calculation of the array is computationally slow and only done once. The multiplication of each ion image in a scan with the conversion-array is a fast matrix operation.

This is a number density, i.e. the number of molecules per speed interval in a unit volume. 
Note that this is a probability density function, i.e. the probability per speed interval.

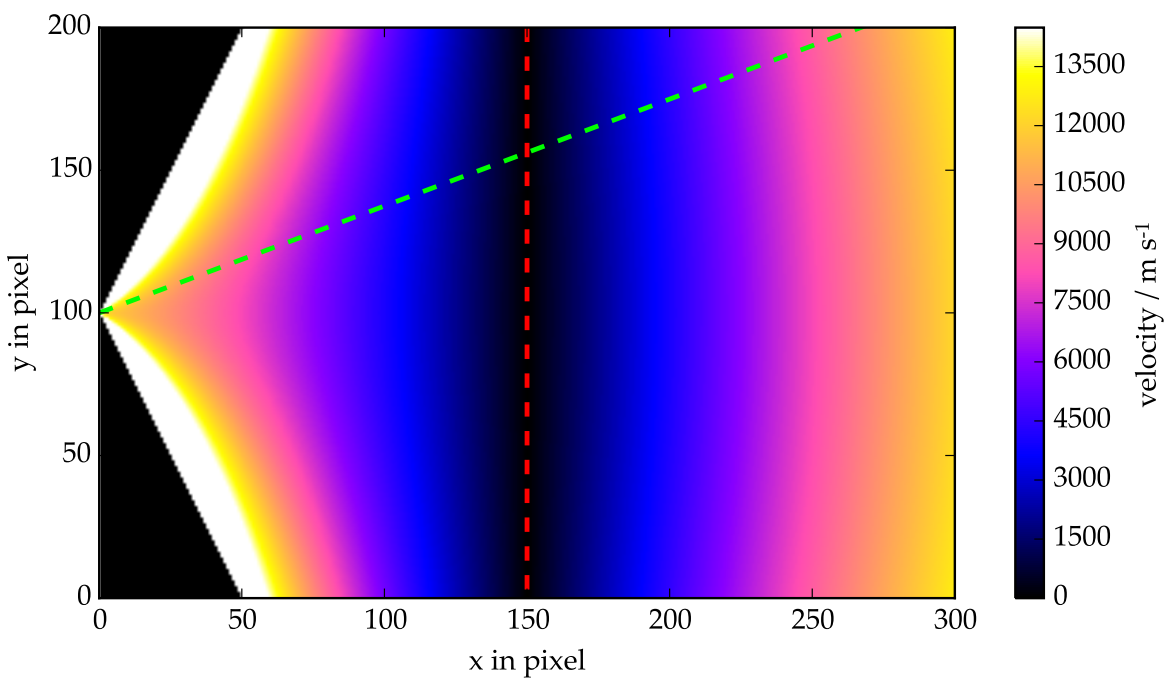

Figure 4.10: Density-to-flux conversion array. The laser is shown as a red, dashed line. The incoming beam trajectory at $22^{\circ}$ from the surface normal is shown as a light-green dashed line.

$$
\mathrm{dn}=\mathrm{n} 4 \pi\left(\frac{\mathrm{m}}{2 \pi \mathrm{k}_{\mathrm{B}} \mathrm{T}}\right)^{3 / 2} \exp \left(-\frac{\mathrm{m} v^{2}}{2 \mathrm{k}_{\mathrm{B}} \mathrm{T}}\right) v^{2} \mathrm{~d} v .
$$

This formula allows us to describe the speed distribution in a volume of ideal gas or in a volume of an effusive beam. I will from now on refer to this as "density speed distribution". It is commonly described as "volume expression" or Comsa et al. [37] refer to this as " $v^{2}$ " $T$ Maxwell distribution. If we want to know the probability of finding a molecule in a given volume with a speed between $v$ and $v+\mathrm{d} v$, we divide equation 4.5 by the volume number density $n$ and obtain the unitless probability as

$$
\mathrm{P}^{\mathrm{d}}(v) \mathrm{d} v=4 \pi\left(\frac{\mathrm{m}}{2 \pi \mathrm{k}_{\mathrm{B}} \mathrm{T}}\right)^{3 / 2} \exp \left(-\frac{\mathrm{m} v^{2}}{2 \mathrm{k}_{\mathrm{B}} \mathrm{T}}\right) v^{2} \mathrm{~d} v
$$

where the superscript ${ }^{d}$ denotes "density".

If, however, we are interested in the speed distribution of molecules impinging on a surface per time, we need a formula describing the flux. With the flux $j=n \times v$ and under the simplifying assumption that all molecules move orthogonally towards the surface we obtain

$$
\mathrm{dj}=\mathrm{dn} \times v=n 4 \pi\left(\frac{\mathrm{m}}{2 \pi \mathrm{k}_{\mathrm{B}} \mathrm{T}}\right)^{3 / 2} \exp \left(-\frac{m v^{2}}{2 \mathrm{k}_{\mathrm{B}} \mathrm{T}}\right) v^{3} \mathrm{~d} v,
$$

which is the flux per speed interval. Of course this equation can also be used to describe the speed of the flux leaving a surface [39]. I will further denote this as "flux distribution". Note the cubic dependence on the speed, which prompts Comsa et al. to refer to this as " $v^{3}$ " T-Maxwell distribution.

If we want to know the probability of a molecule impinging on a surface to have a speed between $v$ and $v+d v$, we have to normalize 
equation 4.7 by dividing by the mean velocity $\langle v\rangle=\left(8 \mathrm{k}_{\mathrm{B}} \mathrm{T} / \pi \mathrm{m}\right)^{1 / 2}$ and divide by $n$ to obtain the probability per speed interval,

$$
P^{f}(v) d v=\frac{1}{2}\left(\frac{m}{k_{B} T}\right)^{2} \exp \left(-\frac{m v^{2}}{2 k_{B} T}\right) v^{3} d v
$$

where the superscript ${ }^{f}$ denotes "flux".

It is important to note that the two distributions are described by different characteristic speeds. The mean speed $\langle v\rangle$ is calculated as

$$
\langle v\rangle=\int_{0}^{\infty} \mathrm{P}(v) v \mathrm{~d} v
$$

and the root mean square speed $\sqrt{\left\langle v^{2}\right\rangle}$ is calculated as

$$
\sqrt{\left\langle v^{2}\right\rangle}=\sqrt{\int_{0}^{\infty} \mathrm{P}(v) v^{2} \mathrm{~d} v}
$$

The most probable, the mean and the root mean square speed for the "density" speed distribution in eq. 4.6 and for the "flux" distribution in eq. 4.8 as found in ref [37] are listed below in table 4.1.

Table 4.1: Characteristic speeds of "density" and "flux" distributions. [37]

\begin{tabular}{lcc}
\hline speeds & "density" & "flux" \\
\hline$v_{\mathrm{mp}}$ (most probable) & $\sqrt{\frac{2 \mathrm{k}_{\mathrm{B}} T}{\mathrm{~m}}}$ & $\sqrt{\frac{3 \mathrm{k}_{\mathrm{B}} T}{\mathrm{~m}}}$ \\
$\langle v\rangle$ (mean) & $\sqrt{\frac{8 \mathrm{k}_{\mathrm{B}} T}{\pi \mathrm{m}}}$ & $\sqrt{\frac{9 \pi \mathrm{k}_{\mathrm{B}} T}{8 \mathrm{~m}}}$ \\
$\sqrt{\left\langle v^{2}\right\rangle \text { (root mean square) }}$ & $\sqrt{\frac{3 \mathrm{k}_{\mathrm{B}} T}{\mathrm{~m}}}$ & $\sqrt{\frac{4 \mathrm{k}_{\mathrm{B}} T}{\mathrm{~m}}}$ \\
\hline
\end{tabular}

The mean square speed $\left\langle v^{2}\right\rangle$ is especially helpful because it is directly proportional to the mean kinetic energy,

$$
\left\langle\mathrm{E}_{\mathrm{kin}}\right\rangle=\frac{1}{2} \mathrm{~m}\left\langle v^{2}\right\rangle,
$$

which leads us to the important insight that the mean kinetic energy in a volume of thermal gas is $\left\langle E_{k i n}\right\rangle=3 / 2 k_{B} T$, while the mean kinetic energy of the thermal flux of molecules leaving a surface at temperature $T_{S}$ is $\left\langle E_{k i n}\right\rangle=2 k_{B} T_{S}$.

Some processes at surfaces, such as recombinative desorption or reactions, can lead to non-thermal speed distributions. Since the speeds are often higher than those of a thermal distribution, we refer to these as hyperthermal distributions. The hyperthermal flux distribution can be described by

$$
\mathrm{df}=\frac{1}{2}\left(\frac{\mathrm{m}}{\mathrm{k}_{\mathrm{B}} \mathrm{T}}\right)^{2} \exp \left(-\frac{\mathrm{m}\left(v-v_{0}\right)^{2}}{2 \mathrm{k}_{\mathrm{B}} \mathrm{T}}\right) v^{3} \mathrm{~d} v,
$$


Note that equation 4.12 is not normalized.

where $v_{0}$ is the offset speed, as reported by Janda et al. [40]. The mean and the root mean square speed for this hyperthermal flux distribution does not have a simple analytical expression and is thus calculated numerically whenever necessary.

Lastly, it can be helpful to examine the energy distribution. To convert the flux speed distribution in equation 4.8 to an energy distribution we replace $\mathrm{v}$ and $\mathrm{dv}$ using $v=\sqrt{2 \mathrm{E} / \mathrm{m}}$ and $\mathrm{d} v=\mathrm{dE} / \sqrt{2 \mathrm{mE}}$ and obtain the probability per energy interval,

$$
P^{f}(E) d E=\left(\frac{1}{2 k_{B} T}\right)^{2} \exp \left(-\frac{E}{k_{B} T}\right) E d E .
$$

For the hyperthermal distribution in equation 4.12 this change of variables yields

$$
\mathrm{df}=\left(\frac{1}{2 \mathrm{k}_{\mathrm{B}} \mathrm{T}}\right)^{2} \exp \left(-\frac{\mathrm{m}\left(\sqrt{2 \mathrm{E} / \mathrm{m}}-v_{0}\right)^{2}}{2 \mathrm{k}_{\mathrm{B}} \mathrm{T}}\right) \mathrm{EdE},
$$

which is also not normalized.

\subsubsection{Speed distributions from ion images}

The calculation of the speed per pixel follows the rules outlined above in section 4.2.3. The most important step during analysis is to choose the correct integration region. The integration regions for a gas-phase process is compared to the one used in imaging of surface processes in figure 4.11. In gas-phase imaging the signal in a sector $d \vartheta$ is in-

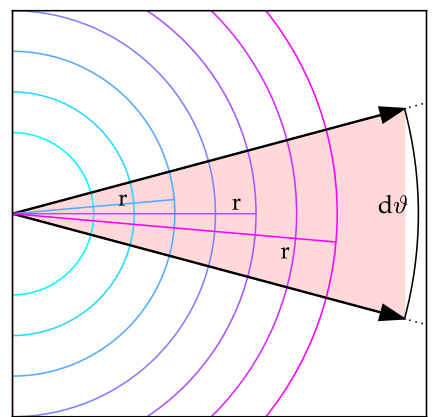

(a) Gas-phase integration

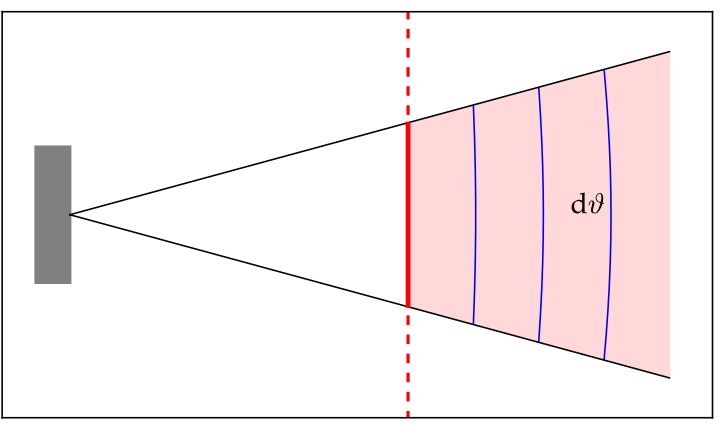

(b) Integration region with surface

Figure 4.11: Comparison of integration regions in gas-phase imaging and in our imaging setup.

tegrated vs the radius $r$, see panel (a). In our experimental setup in panel (b) the molecules are ionized at the laser focus indicated by

Further information on the implementation in the analysis code can be found in the appendix, section B.1. the solid red line. In both cases fast ions move further away from the source/laser and spread over a larger area (along y), but occupy the same $d \vartheta$. The integration regions (red shaded areas) also scale proportionally. The integration region as shown in panel (b) extends further 
to the right, i.e. to higher speeds than observed, to capture all ions. This is also useful for a proper baseline correction.

\subsection{IMAGE PROCESSING}

Since the ion images generated at the Beamer II machine are unique in several ways, we developed new analysis methods. Especially the extraction of kinetic information can be done in different ways. In this section I will introduce the different methods.

\subsubsection{ROI vs delay}

The first and most simple approach we developed to extract kinetic data was the integration of a region of interest (ROI) in each ion image vs the beam-laser delay at which the image was recorded. Initially we used a Fortran command-line utility with the range along $\mathrm{x}$ and $\mathrm{y}$ specified in the code. Since this was inconvenient as it did not allow the integration region to be adjusted visually, we soon switched to a graphical user interface based on Python codes. After choosing the integration region ("box") the code simply loops over all image files in a directory and calculates the average signal in the box for each file. The delay of each image file is extracted from the header. This yields the average density signal in the box vs beam-laser delay. It is possible to multiply each ion image with a density-to-flux conversion array prior to integration. This yields the average flux in the box vs beam-laser delay.

The experimentally recorded data are the raw ion images and these are saved for later analysis. We can therefore go back and extract different regions if necessary. By using a narrow box a small velocity range can be selected. This allows the measurement of the velocity resolved flux.

\subsubsection{Background signal correction}

In some experiments signal from background gas complicates the measurements of kinetics. If the background gas has a steady concentration, its contribution can be subtracted by recording a dark image. In a kinetic scan the constant contribution would just change the value of the baseline. If, however, the background gas concentration is modulated by the incoming beam, this biases the shape of the signal vs beam-laser delay. We therefore implemented a correction method to remove the background contribution in each ion image.

The upper left panel in figure 4.12 shows a density ion image of $\mathrm{CO}_{2}$ produced at $\mathrm{Pt}(111)$ with three components with different velocities. The signal from background gas is assumed to have a uniform velocity distribution with the laser in the center. The upper right cor-

The dark image is subtracted from each ion image during recording.
Note that these routines extract the average signal. This facilitates the comparison of boxes of different size.

It is recommended to crop ion images to the relevant section to reduce the file size. 

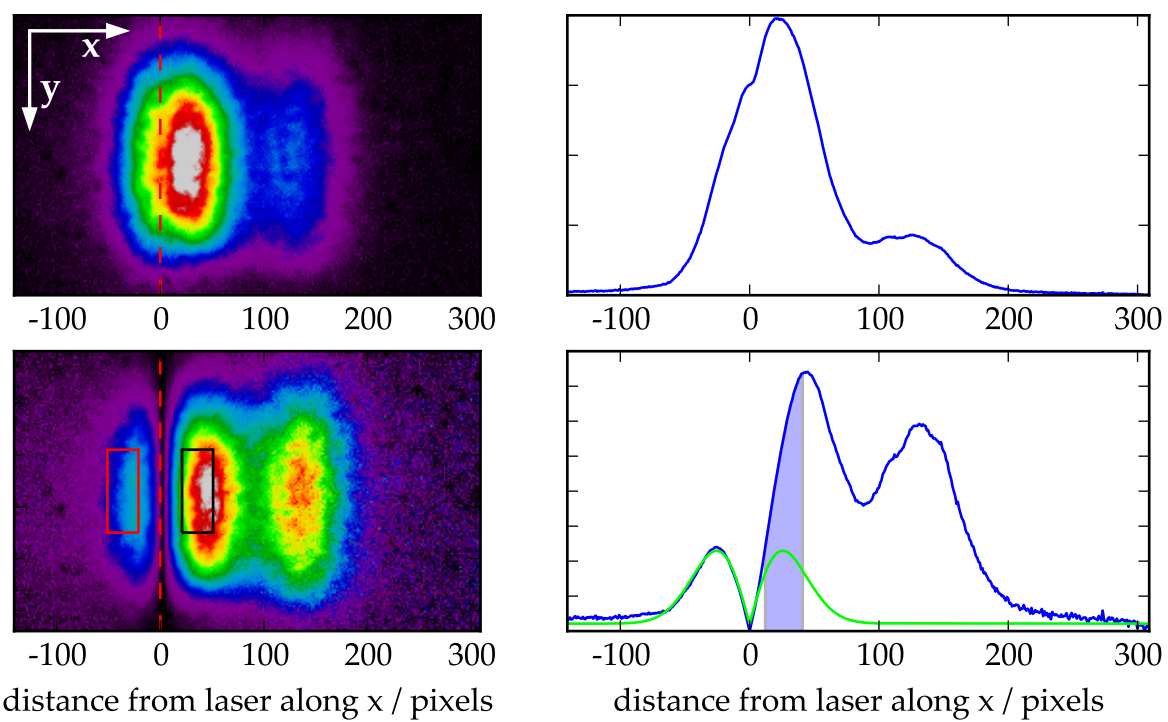

Figure 4.12: Correction of background signal in kinetic scans: The top panels show an ion image and its histogram. The lower panels show the same data after conversion to flux. The laser position is indicated by the red dashed line. The background signal in the lower right panel is indicated by the green line, see text.

ner shows a histogram of the signal intensity along $\mathrm{x}$. Upon close inspection a shoulder centered around the laser at $x=0$ pixels can be seen. The main peak near the laser in the ion image also looks slightly asymmetric. All $\mathrm{CO}_{2}$ molecules produced at the $\mathrm{Pt}(111)$ surface, which is located at about $x=-400$ pixels, should be moving away from the surface and thus appear to the right of the laser. Any intensity on the left of the laser is contribution from background gas, e.g. $\mathrm{CO}_{2}$ scattered from the ion optic grids. The lower panels show the same ion image and histogram after conversion of density to flux.

Consider integrating the signal in the black ROI in the lower left panel. The signal in this black box is equivalent to the blue area under the curve in the lower right panel. When mirroring the black box with respect to the laser, we obtain the red box on the left. We can see that there is significant background gas contribution in this red box. Under the assumption that the background gas is uniformly distributed around the laser, there must be an equal amount of background signal in the black box as well. We can visualize this contribution by fitting the signal at negative $x$ with a $1 D$ flux Maxwell-Boltzmann function as plotted in green.

In order to remove the background signal contribution we mirror the ion signal inside the red box and subtract it from the black box

Avoid cropping the ion images too close to the laser if this correction method shall be used. when processing the ion images. One way to picture this is folding the ion image in the lower left panel around the laser. The red and black box would be exactly on top of each other and the background signal would cancel out when subtracting the image in the red box from the image in the black box. 


\subsubsection{Kinetic rates versus speed}

A different approach to extract kinetic rates from the $\mathrm{CO}$ oxidation data is to fit the two peaks observed in the speed distribution individually at each delay. A sum of a Maxwell-Boltzmann distribution and a hyperthermal distribution is fitted to each ion image and the area of the two contributions is plotted as a function of beam-laser delay. The advantage of this approach compared to the simple and straightforward box integration is the correct scaling of the two peaks. Since the fits require the ion signal at each beam-laser delay to resemble a Maxwell-Boltzmann or hyperthermal distribution, this approach requires data with a much higher signal-to-noise ratio (SNR). Another disadvantage is that the flight time dependence on the speed is not taken into account.

With these limitations in mind, another approach combining the simple box integration with the fit to the speed distribution was developed. The ion image is split into several small boxes as shown in figure 4.13 in the upper panel. For each of these boxes the mean TOF can be calculated and used as an offset when fitting the kinetic traces. For this example (CO oxidation on $\mathrm{Pt}(111), \mathrm{T}_{\mathrm{S}}=603 \mathrm{~K}, 100 \mathrm{~Hz} \mathrm{CO}$ beam, $200 \mathrm{~Hz} \mathrm{O}_{2}$ beam, see chapter 6) the extracted data was fitted arbitrarily with a bi-exponential decay $\left(\tau_{\text {slow }}=351 \mu \mathrm{s}\right.$ and $\left.\tau_{\text {fast }}=34 \mu \mathrm{s}\right)$ where the lifetime was a shared parameter of all boxes and the amplitude was allowed to vary in each box.
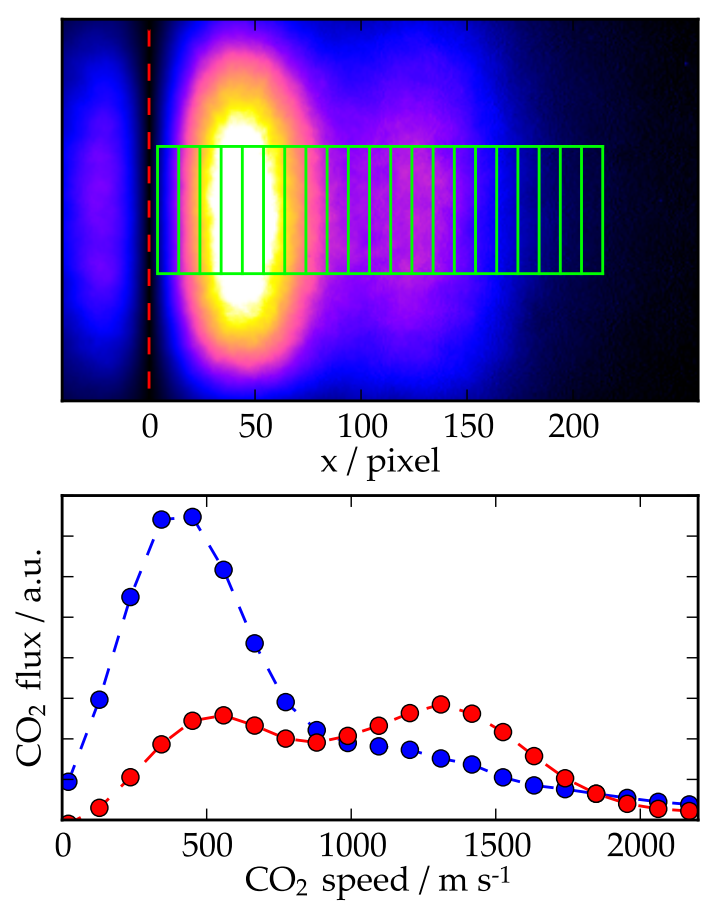

Figure 4.13: Multiple box approach: The top panel shows a sum image of $\mathrm{CO}$ oxidation on $\operatorname{Pt}(111)$ at ${ }_{60} \mathrm{~K}$ and an RRR of 2 . See chapter 6 for more information on this scan. The image is split into several boxes with finite size along $x$ and the kinetic trace is extracted for each box. The lower panel shows the result of fitting each kinetic trace with a bi-exponential decay with $\tau_{\text {slow }}=351 \mu \mathrm{s}$ and $\tau_{\text {fast }}=34 \mu \mathrm{s}$. The contribution of the slow decay is plotted in blue, the fast decay in red.

The contribution of the fast and slow decay to each kinetic trace is plotted in the lower panel of figure 4.13. Independent of whether the bi-exponential decay is a viable representation of the kinetics, we

This section uses some of the $\mathrm{CO}$ oxidation data presented in chapter 6. Please see the later chapter for detailed information.

The scaling does not include angular distributions. 
can learn something important from the lower panel - there is a significant contribution of the fast decay at slow velocities. The fast decay shows a bimodality in velocity.

\subsection{VELOCITY-MAP IMAGING OF PROCESSES AT SURFACES}

The measurements and techniques above all relied on spatial imaging but there are some situations where velocity-map imaging can greatly facilitate the experiment or is even a requirement. Collapsing the spatial extent provides higher resolution in the velocity distribution, provided that the flight path is long enough to achieve an adequate ion image size.

This effect also applies to angular distributions. The beam diameter on the surface of $3 \mathrm{~mm}$ or the surface diameter itself $(9 \mathrm{~mm})$ are not small compared to the $20-30 \mathrm{~mm}$ long region where ions are typically detected in angular distribution measurements. This leads to erroneously broad angular distributions in spatial imaging but can be avoided using VMI.

An experiment which can hardly be done without velocity-map imaging would be the vibrationally resolved scattering of nitrous oxide from a surface. Photodissociation of the scattered $\mathrm{N}_{2} \mathrm{O}$ and $2+1$ REMPI of the $\mathrm{N}_{2}$-fragment allows the vibrational distribution of the scattered $\mathrm{N}_{2} \mathrm{O}$ to be resolved. The interaction length of the laser with the scattered gas would be large compared to the possible separation of the fragment ions in spatial imaging. The vibrational state distribution of the parent molecule would thus be concealed unless VMI is used.

\section{REFERENCES}

[33] H. Niehus and B. Krahl-Urban. "Improved technique in electron stimulated desorption ion angular distributions." In: Review of Scientific Instruments 52.1 (1981), pp. 56-59. DOI: 10 . 1063/1.1136446 (cit. on pp. 39, 40).

[34] Paul H. Kobrin, G. Alan Schick, James P. Baxter, and Nicholas Winograd. "Detector for measuring energy- and angle-resolved neutral-particle (EARN) distributions for material desorbed from bombarded surfaces." In: Review of Scientific Instruments 57.7 (1986), pp. 1354-1362. DoI: 10.1063/1.1138600 (cit. on p. 39).

[35] Dan J. Harding, Jannis Neugebohren, Daniel J. Auerbach, T. N. Kitsopoulos, and Alec M. Wodtke. "Using Ion Imaging to Measure Velocity Distributions in Surface Scattering Experiments." In: Journal of Physical Chemistry A 119.50 (2015), pp. 1225512262. DoI: 10.1021/acs . jpca. 5 b06272 (cit. on p. 43). 
[36] Kenichi Tonokura and Toshinori Suzuki. "Slicing photofragment spatial distribution by laser sheet ionization." In: Chemical Physics Letters 224.1-2 (1994), pp. 1-6. DoI: 10.1016/00092614 ( 94 ) 00533 - 8 (cit. on p. 44).

[37] George Comsa and Rudolf David. "Dynamical parameters of desorbing molecules." In: Surface Science Reports 5.4 (Dec. 1985), pp. 145-198. DOI: 10 . $1016 / 0167$ - 5729 (85 ) 90009 - 3 (cit. on pp. $45,46,50,51,131$ ).

[38] C.T. Campbell, G. Ertl, H. Kuipers, and J. Segner. "A molecular beam investigation of the interactions of $\mathrm{CO}$ with a $\mathrm{Pt}(111)$ surface." In: Surface Science Letters 107.1 (May 1981), A222. DOI: 10.1016/0167-2584 (81) 90554- 5 (cit. on pp. 46, 62, 100).

[39] Otto Stern. "Nachtrag zu meiner Arbeit: Eine direkte Messung der thermischen Molekulargeschwindigkeit." In: Zeitschrift für Physik 3 (1920), pp. 417-421 (cit. on p. 50).

[40] Kenneth C. Janda, Jerry E. Hurst, Charles A. Becker, James P. Cowin, Daniel J. Auerbach, and Lennard Wharton. "Direct measurement of velocity distributions in argon beam-tungsten surface scattering." In: The Journal of Chemical Physics 72.4 (1980), p. 2403. DOI: 10.1063/1.439434 (cit. on pp. 52, 80). 



\section{Part II}

\section{CO OXIDATION ON PLATINUM SURFACES}

In this chapter I present investigations on the various processes involving carbon monoxide on platinum surfaces conducted at Beamer II.

The trapping-desorption on $\mathrm{Pt}(111)$ and (332) is described in chapter 5 . In chapter 6 I show how the kinetic model describing the oxidation of $\mathrm{CO}$ was infered from experimental observations. The results and discussion are presented in chapter 7 and 8 . 



\subsection{TRAPPING-DESORPTION ON SURFACES}

The trapping and subsequent desorption of molecules on a singlecrystal metal surface can be investigated by various methods. In contrast to temperature programmed desorption (TPD), where the desorption is monitored as a function of temperature and typical coverages $\theta$ are between 0.1-1 ML, molecular beam methods enable timeresolved, isothermal measurement at low coverages. The time resolution is useful in the investigation of the underlying kinetics and low coverages allow adsorbate interactions to be avoided. The desorption is a first order process and the exponential decay of the flux of desorbing molecules can be measured using e.g. laser-based detection or a QMS time-of-flight detector.

In a first-order desorption process, the flux of molecules leaving the surface can be mathematically described as a convolution of the incoming beam function with an exponential decay. The measured flux, however, is also a function of the flight time from the surface to the detector. Since a fast molecule needs less time ( $\left.t_{\mathrm{TOF}}^{\text {fast }}\right)$ to travel to the detector than a slow molecule $\left(t_{\mathrm{TOF}}^{\text {slow }}\right)$, the flux $J_{\text {detector }}(t)$ measured at the detector does not represent the flux of molecules leaving the surface at a certain time $J_{\text {surface }}\left(t-t_{T O F}\right)$, but rather a sum over the speed-dependent desorption times:

$$
\mathrm{J}_{\text {detector }}(t)=\sum_{\text {speed }} J_{\text {surface }}\left(t-t_{\text {TOF }}^{\text {speed }}\right)
$$

In order to measure the flux of desorbing molecules as a function of time, one has to selectively detect molecules of a single speed. In timeof-flight experiments this can be achieved by using several chopper wheels in front of the detector.

An alternative technique using two lasers has been demonstrated by Golibrzuch et al. [41]. One laser was used to tag molecules close to the surface and a second laser ionized the tagged molecules further down the flight path, selecting a velocity. By scanning the molecular beam-laser delay, the flux of molecules desorbing from the surface as a function of time is obtained. By fitting a convolution of the incoming beam shape with an exponential decay to this flux, the mean residence time on the surface can be determined. An inherent consequence of this procedure is that the temporal resolution of the experiment is limited by the width of the incoming beam pulses.

Another approach is available when combining surface reactions with ion imaging. In our experimental setup the relevant velocity is 
The step density was determined in section 2.2. parallel to the imaging detector, see section 4.1.2. A velocity-selected flux can be obtained by measuring the signal in a selected area on the detector as a function of beam-laser delay, see section 4.3.1. It is worth noting that our imaging technique using a single laser can be applied to any molecule, whereas the two-laser tagging technique requires a long-lived and accessible excited state.

\subsection{TRAPPING-DESORPTION OF CO ON PT(III)}

In order to both establish experimental and analytic routines and to prepare for the subsequent investigation of $\mathrm{CO}$ oxidation on platinum, the trapping-desorption (TD) of $\mathrm{CO}$ on $\mathrm{Pt}(111)$ was investigated at Beamer II. The recent study by Golibrzuch et al. [41] served as a reference point for our experiment.

A molecular beam of $\mathrm{CO}$ incident at $22^{\circ}$ was used to deposit up to $0.05 \mathrm{ML}$ of $\mathrm{CO}$ on the crystal surface, where $0.50 \mathrm{ML}$ is the maximum coverage of $\mathrm{CO}$ on $\mathrm{Pt}(111)$ [38]. The experiment thus took place at low coverages where adsorbate-adsorbate interactions are negligible. Both a pure $\mathrm{CO}$ beam and a seeded beam $(5 \% \mathrm{CO}$ in $\mathrm{He}$ ) were used, yielding the same result. The seeded beam had a more favorable pulse shape, which was typically $\sim 25 \mu$ sWHM, which allowed for measurements at higher temperatures. The $\mathrm{CO}$ dose per pulse is much higher than the available step sites of the $\mathrm{Pt}(111)$ crystal.

A small fraction of the incident $\mathrm{CO}$ molecules exhibited direct scatter along the specular angle, while the majority of the $\mathrm{CO}$ became trapped on the surface before undergoing thermal desorption in a cosine distribution with thermal velocity, as seen in figure 5.I.

By integrating over a velocity-selected region of each image, the time-dependent flux is obtained. Kinetic traces for several temperatures are shown in figure 5.2. Each kinetic trace contains contributions from trapping-desorption and direct scattering. For the top trace at $603 \mathrm{~K}$ the desorption is relatively slow. The trapping-desorption signal therefore stretches over several $\mathrm{ms}$ and the contribution of direct scattering is easily distinguished from the contribution of direct scatter. The black trace on the bottom of the graph shows the $\mathrm{CO}$ flux from the surface at $973 \mathrm{~K}$. Since the residence time of $\mathrm{CO}$ on the surface at this temperature is on the sub- $\mu$ s scale, i. e. below our experimental resolution and far below the incoming beam pulse width, we estimate the shape of this trace to be similar to the direct scatter contribution at the beginning of the blue trace at $603 \mathrm{~K}$. 


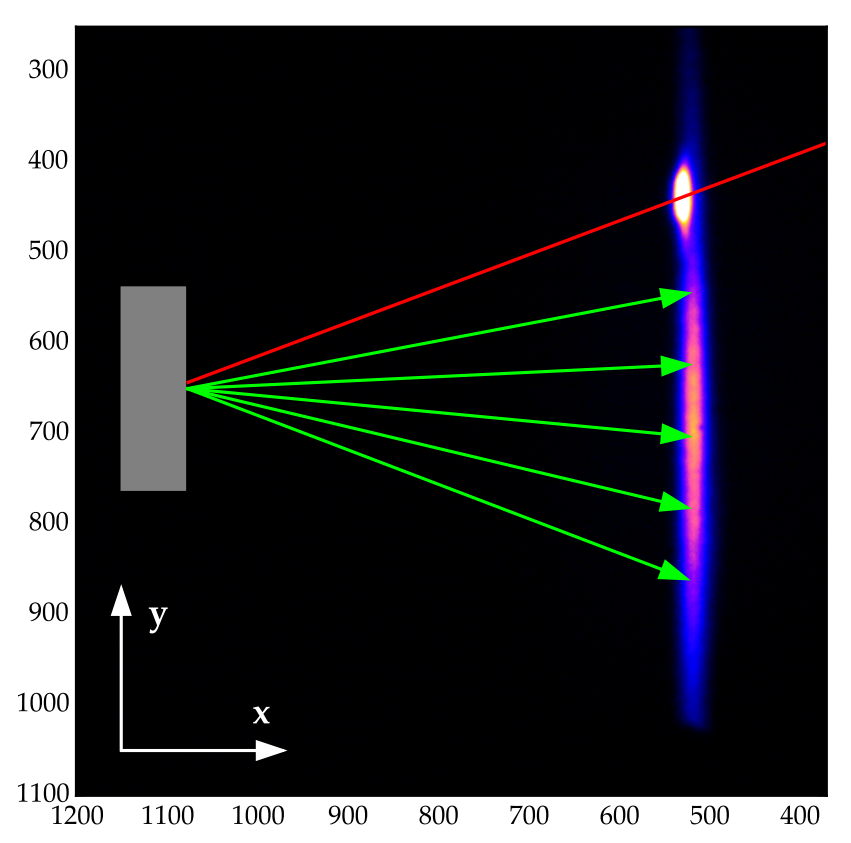

Figure 5.1: Ion image of $\mathrm{CO}$ : Ions from the incident $\mathrm{CO}$ beam can be seen on top. The direction of the incident beam is indicated in red and the position of the $\mathrm{Pt}(111)$ surface is indicated by the gray rectangle. The apparent angular distribution of the ions is narrower than a cosine distribution due to the intensity variation along the focal length of the $2+1$ REMPI laser.

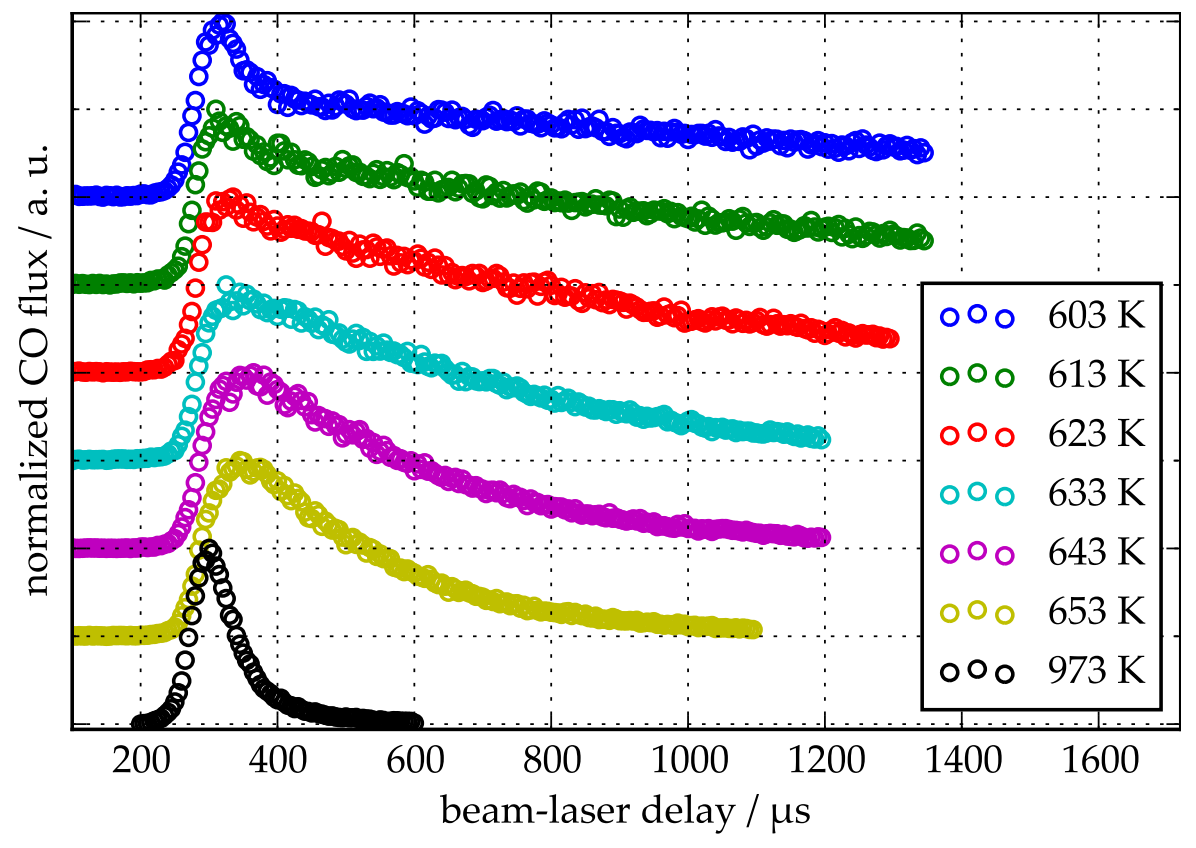

Figure 5.2: Velocity selected flux of $\mathrm{CO}$ for several temperatures. The traces are normalized and shifted along y for better visibility. The surface temperature for each trace is shown in the legend. 


\subsubsection{Kinetic equations}

The mean desorption time, the lifetime on the surface, is defined by the rate coefficient for desorption $k_{d}$ as $\tau_{d}=1 / k_{d}$. This process is described by

$$
\mathrm{CO}_{\mathrm{a}} \stackrel{\mathrm{k}_{\mathrm{d}}}{\longrightarrow} \mathrm{CO}_{\mathrm{g}}
$$

where a denotes adsorbed and g denotes gaseous $\mathrm{CO}$. The rate of $\mathrm{CO}$ desorption is

$$
\frac{\mathrm{d}\left[\mathrm{CO}_{\mathrm{a}}\right]}{\mathrm{dt}}=-\mathrm{k}_{\mathrm{d}}\left[\mathrm{CO}_{\mathrm{a}}\right],
$$

and the concentration of adsorbed $\mathrm{CO}_{\mathrm{a}}$ at a time $\mathrm{t}$ is derived as

$$
\begin{aligned}
\int_{\left[\mathrm{CO}_{a}\right]_{0}}^{\left[\mathrm{CO}_{a}\right]_{t}} \frac{\mathrm{d}\left[\mathrm{CO}_{\mathrm{a}}\right]}{\left[\mathrm{CO}_{\mathrm{a}}\right]} & =-\mathrm{k}_{\mathrm{d}} \int_{0}^{\mathrm{t}} \mathrm{dt} \\
\ln \frac{\left[\mathrm{CO}_{\mathrm{a}}\right]_{t}}{\left[\mathrm{CO}_{\mathrm{a}}\right]_{0}} & =-k_{d} \mathrm{t} \\
{\left[\mathrm{CO}_{\mathrm{a}}\right]_{t} } & =\left[\mathrm{CO}_{\mathrm{a}}\right]_{0} \exp \left(-\mathrm{k}_{\mathrm{d}} \mathrm{t}\right) .
\end{aligned}
$$

Using Eq. 5.3 and 5.4 we can express the flux of desorbing $\mathrm{CO}$ as

$$
\begin{aligned}
\operatorname{Flux}\left(\mathrm{CO}_{g}\right) & =k_{d}\left[\mathrm{CO}_{a}\right]_{t} \\
& =k_{d}\left[\mathrm{CO}_{a}\right]_{0} \exp \left(-k_{d} t\right),
\end{aligned}
$$

where $\left[\mathrm{CO}_{\mathrm{a}}\right]_{0}$ is the amount of $\mathrm{CO}$ deposited at the beginning of the process.

\subsubsection{Convolution with the incoming beam}

Equation 5.5 can also be written as

$$
f(t)=B \times \exp \left(-\frac{t}{\tau_{d}}\right),
$$

with $\tau_{d}=1 / k_{d}$ and $B=k_{d}\left[\mathrm{CO}_{a}\right]_{0}$. Since $\mathrm{CO}_{a}$ is not deposited instantly, we have to account for the width of the incoming $\mathrm{CO}$ beam. The incoming beam profile is modelled by the sum of N Gaussians

$$
G_{N}(t)=\sum_{\alpha=1}^{N} g_{\alpha}(t)
$$

with

$$
g_{\alpha}(t)=C_{\alpha} \times \exp \left(-\frac{\left(t-t_{0, \alpha}-t_{0}\right)^{2}}{\sigma_{\alpha}^{2}}\right),
$$

where $C_{\alpha}$ is the amplitude, $t_{0, \alpha}$ the shift of the Gaussian functions relative to one another and $t_{0}$ the shift of the total function on the 
time axis. A convolution of the incoming beam fit function in Eq. 5.7 with the exponential decay in Eq. (5.6) yields the desorption flux from the surface

$$
J_{N}(t)=\int_{0}^{\infty} f\left(t^{\prime}\right) G_{N}\left(t-t^{\prime}\right) d t^{\prime}=\sum_{\alpha} j_{\alpha}(t)
$$

The convolution

$$
j_{\alpha}(t)=\int_{0}^{\infty} f\left(t^{\prime}\right) g_{\alpha}\left(t-t^{\prime}\right) d t^{\prime}
$$

can be calculated analytically yielding

$$
j_{\alpha}(t)=A_{\alpha} \exp \left(\frac{\sigma_{\alpha}^{2}-4\left(t-t_{0, \alpha}-t_{0}\right) \tau_{d}}{4 \tau_{d}^{2}}\right) \operatorname{erfc}\left(\frac{\sigma_{\alpha}}{2 \tau_{d}}-\frac{t-t_{0, \alpha}-t_{0}}{\sigma_{\alpha}}\right)
$$

with

$$
A_{\alpha}=\frac{\sigma_{\alpha} \sqrt{\pi}}{2} C_{\alpha} B
$$

This function was used to fit the $\mathrm{CO}$ flux as a function of time as shown in figure $5 \cdot 3$.

\subsubsection{Results - CO on Pt(111)}

A series of $\mathrm{CO}$ flux vs time at different temperatures and the respective fits are shown in figure 5.3. The data in the upper panel is the same as in figure 5.2. The measured $\mathrm{CO}$ flux from the surface consists of both desorbing $\mathrm{CO}$ and directly scattered CO. Especially at lower temperatures the direct scattering contribution is clearly distinguishable. To account for this, the kinetic trace at $973 \mathrm{~K}$ surface temperature is fit with a convolution as described in equation 5.11. The result was included as a fixed component in the other fits, only the amplitude was allowed to vary. An example is shown in panel (b): The trapping-desorption data at $603 \mathrm{~K}$ shows a distinct contribution from direct scattering fitted by the dashed, black curve. The remainder, mostly the data at later times, was fit with another convolution (green, dashed).

When comparing non-normalized plots at various temperatures, one can see that the contribution of directly scattered $\mathrm{CO}$ barely changes, while the trapping-desorption signal varies in both lifetime and amplitude. The position of the laser focus and laser intensity was not changed across temperatures, therefore the ratio of the areas (time-integrated flux) should stay the same as long as the sticking probability does not change significantly. The time-integrated $\mathrm{CO}$ desorption flux is obtained by calculating the area under the dashed, green line in panel (b) of figure 5.3 and plotted in panel (c). The total

The desorption lifetime is very small at $973 \mathrm{~K}$. Compared to the incident beam the shape is slightly broadened due to the TOF acceptance range of the ROI. This broader shape can be approximated by a convolution with $\tau_{\mathrm{d}}=25 \mu \mathrm{s}$. 


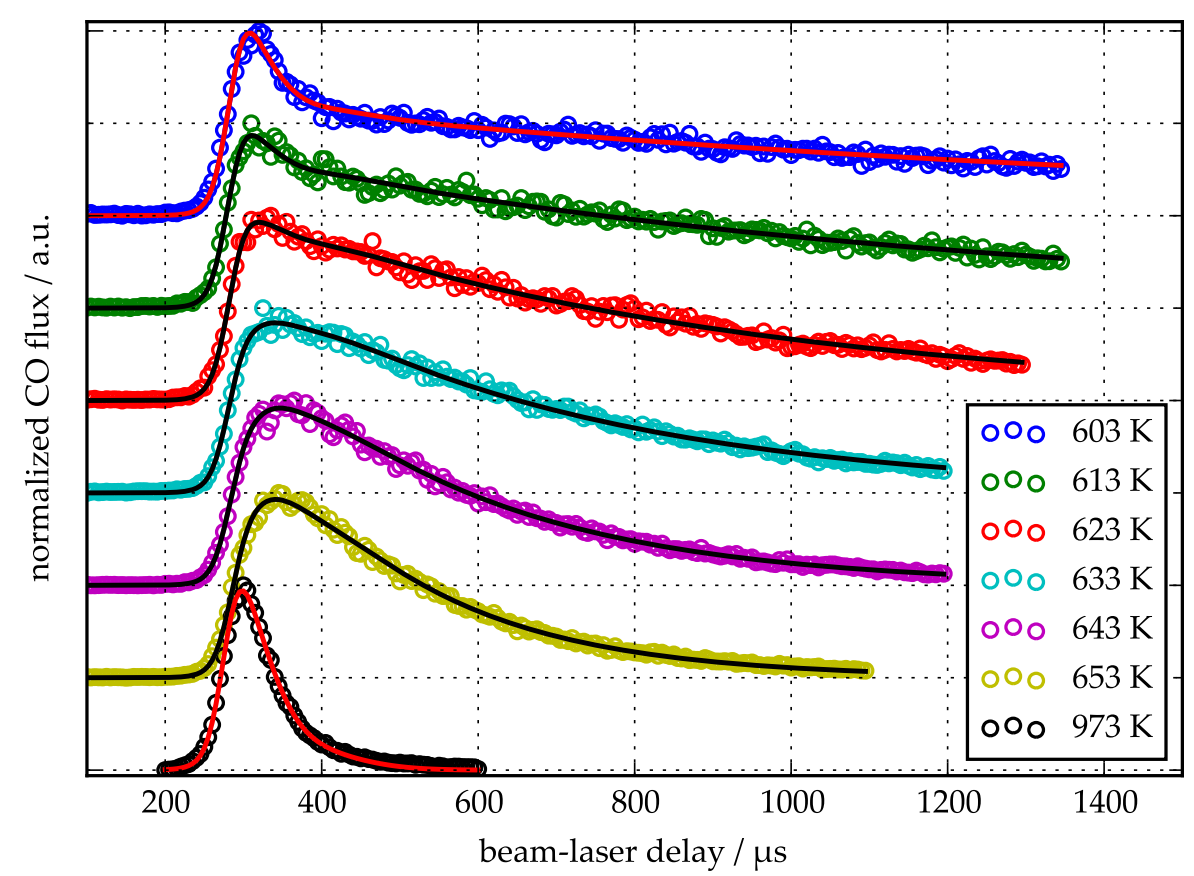

(a) velocity-selected $\mathrm{CO}$ desorption flux with fits

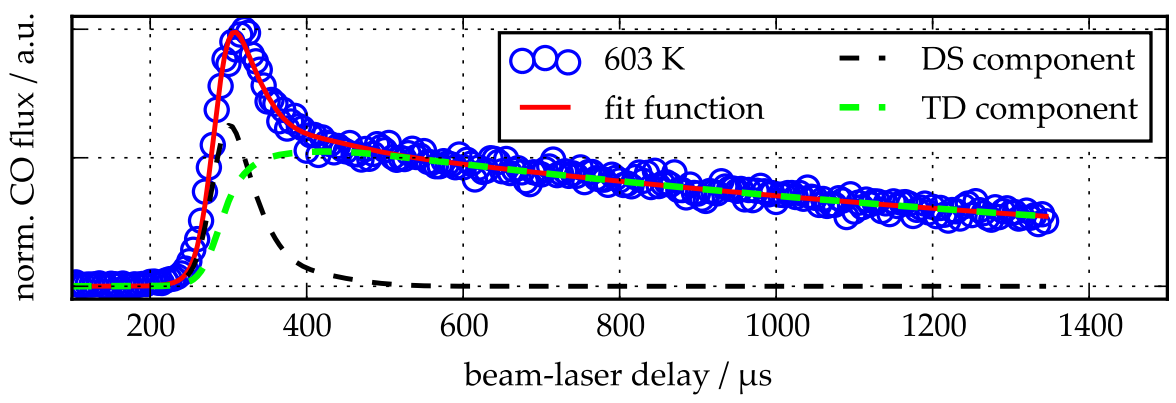

(b) fit contributions of the direct scatter (DS) and desorption (TD) channel

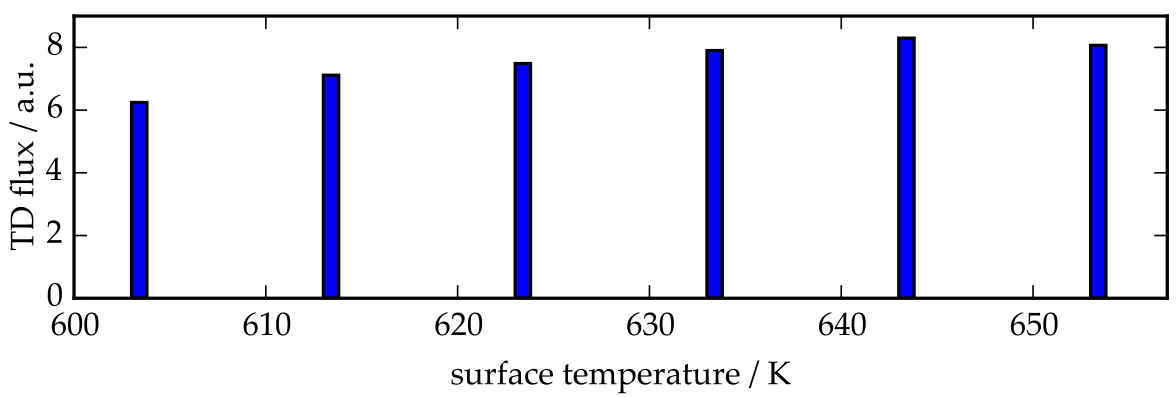

(c) time-integrated $\mathrm{CO}$ desorption flux

Figure 5.3: Panel (a): The data from figure 5.2 with fits. The respective surface temperatures are given in the legend. Panel (b): The kinetic trace at $603 \mathrm{~K}$, the fit function (red) and the different contributions to the fit (black and green, dashed). Panel (c): The time-integrated desorption flux, i. e. the total desorption signal vs temperature. 
amount of desorbing $\mathrm{CO}$ is almost the same across the different temperatures. The slight increase with temperature might be due to the detection efficiency - the discrimination between desorbing $\mathrm{CO}$ and background signal is facilitated at higher desorption speeds.

An Arrhenius analysis, which is a linear regression of $\ln \left(\mathrm{k}_{\mathrm{d}}\right)$ vs inverse temperature, allows the activation energy and the pre-exponential factor characterizing the desorption process to be extracted. An Arrhenius plot is shown in figure 5.4. The averaged result from several

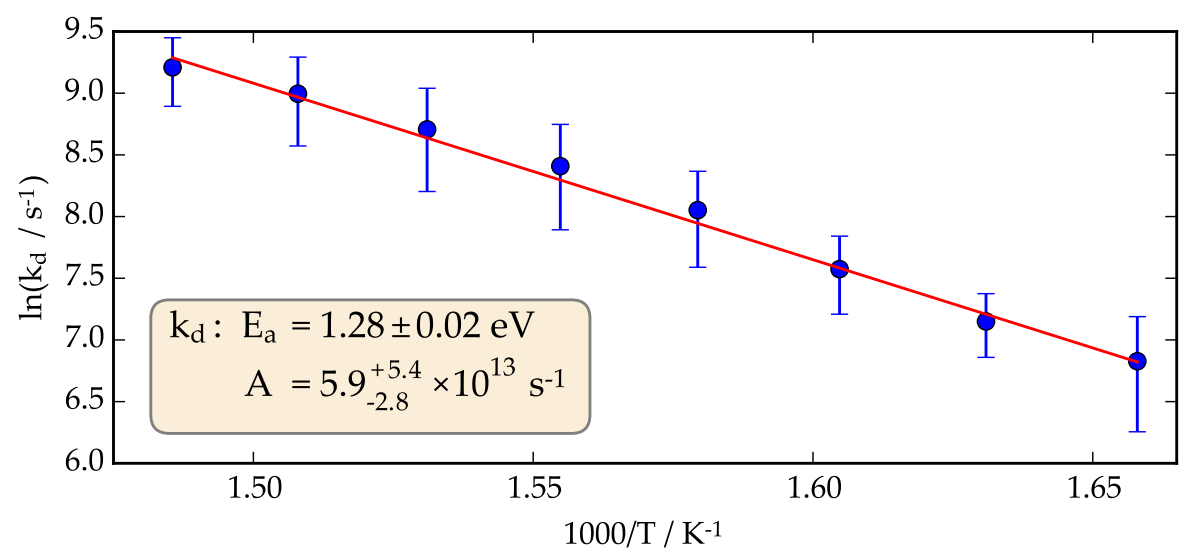

Figure 5.4: Arrhenius plot of $\mathrm{CO}$ trapping-desorption rates on $\mathrm{Pt}(111)$. An error-weighted linear fit is shown as a red line.

datasets, shown as an inset, agrees well with the recently determined value for trapping-desorption on $\mathrm{Pt}(111)$ terraces by Golibrzuch et al. $\left(E_{a}=1.27 \pm 0.07 \mathrm{eV}, \mathrm{A}=3.5_{-2.7}^{+7.2} \times 10^{13} \mathrm{~s}^{-1}\right)$. While a comparison of activation energy and pre-exponential factor is straightforward, a direct comparison of rate coefficients is less error-prone. Such a comparison is presented at the end of this chapter.

\subsection{TRAPPING-DESORPTION OF CO ON PT(332)}

As described in section 2.2 the $\mathrm{Pt}$ (332) surface has one (111)-step per five (111)-terrace atoms, resulting in a step density of $16 \%$. On $\mathrm{Pt}(111)$ the $\mathrm{CO}$ exposure is much greater than the concentration of steps. The dominant process on the atomically flat $\mathrm{Pt}(111)$ surface would then be the desorption from terrace sites. On $\mathrm{Pt}(332)$ the exposure is much less than the concentration of steps and as the diffusion range on terraces is large at the temperatures investigated, every $\mathrm{CO}$ atom is expected to bind to a step site at least once after adsorption. The desorption on $\mathrm{Pt}(332)$ is thus expected to be dominated by an activation energy and pre-exponential factor corresponding to a step-site assisted desorption process. A kinetic model involving a step-site assisted desorption process has been formulated by Serri, Tully and Cardillo [42] for desorption of NO from Pt(111). They argued that the desorption from steps is a sequential process consisting of diffusion

The diffusion range is discussed in detail in section 8.1.4. 
to terraces followed by desorption from terraces. The measured desorption rate coefficient would then be an effective rate coefficient for the whole process. Golibrzuch et al. applied this model to the desorption of $\mathrm{CO}$ from $\mathrm{Pt}(111)$.

\subsubsection{Results: CO on Pt(332)}

Kinetic traces of trapping-desorption from $\mathrm{Pt}(332)$ are shown in figure 5.5a. The temperatures are given in the legend and increase from top to bottom. Compared to the trapping-desorption on $\mathrm{Pt}(111)$ the temperature range investigated here is $60-90 \mathrm{~K}$ higher. For similar temperatures, the desorption rates from steps are much slower. Apart from the trapping-desorption (TD) signal, a direct-scatter (DS) contribution can be observed and is fitted by the incoming $\mathrm{CO}$ beam shape. The fit function is a combination of the incoming beam shape and a convolution of an exponential decay over the incoming beam shape as defined in equation 5.11. As an example the fit contributions to the kinetic trace at $683 \mathrm{~K}$ are shown in panel (b) of figure 5.5. Compared to the desorption lifetime at this temperature the direct-scatter component is very short.

An Arrhenius analysis of the desorption rate coefficient on $\operatorname{Pt}(332)$ is shown in panel (c), the result is annotated in the box. The data with standard deviation shown here is averaged over multiple datasets. 


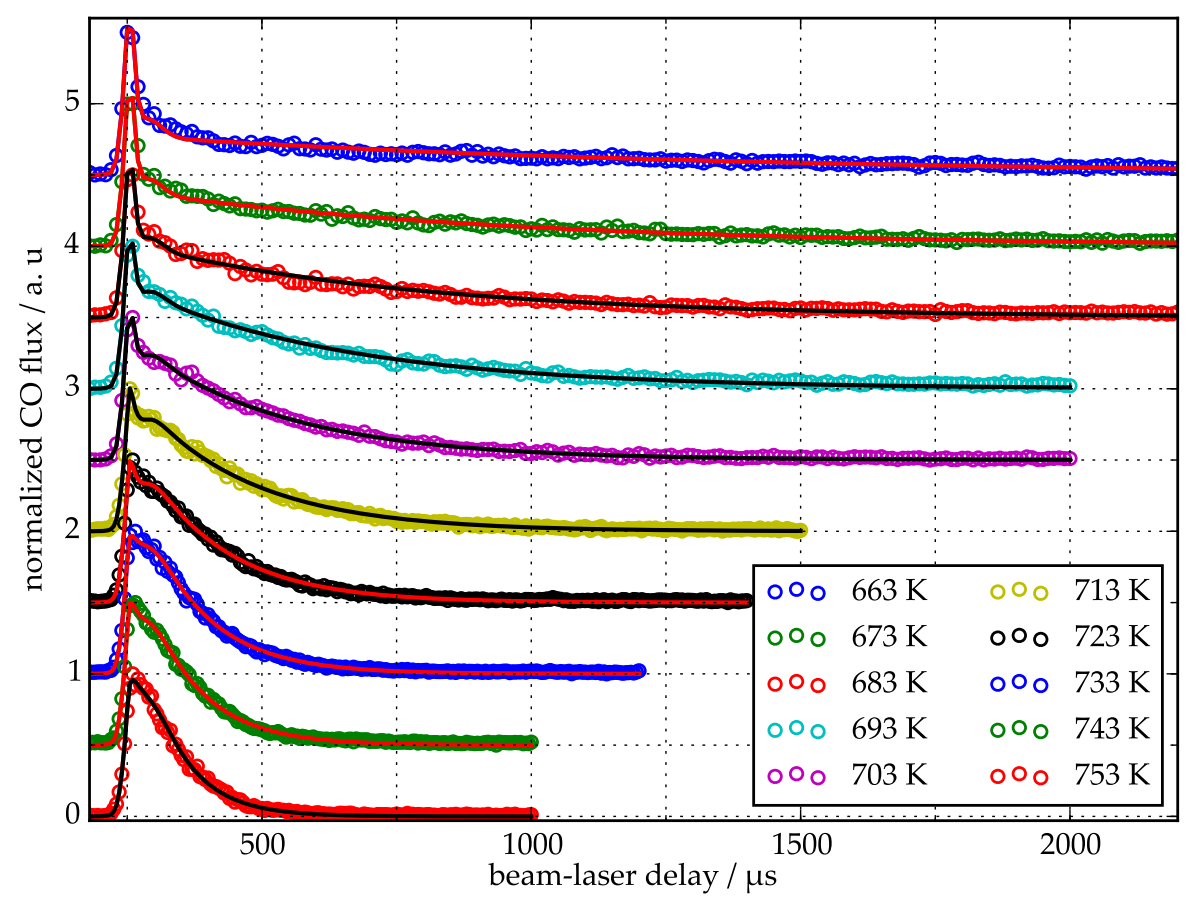

(a) velocity-selected $\mathrm{CO}$ desorption flux with fits

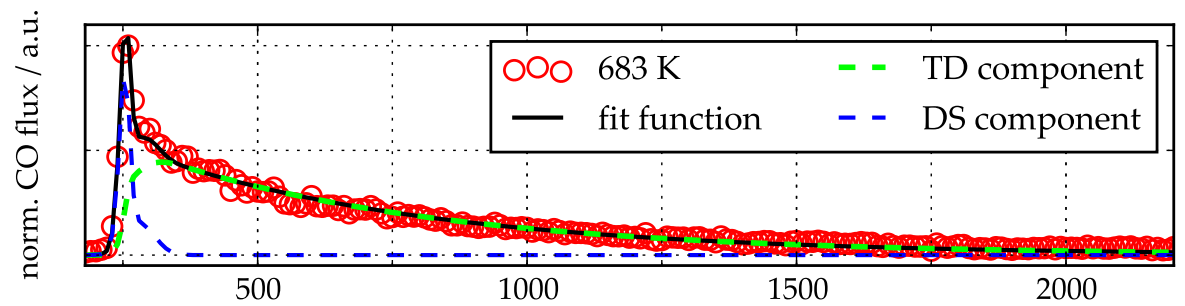

(b) fit contributions of the direct scatter (DS) and desorption (TD) channel

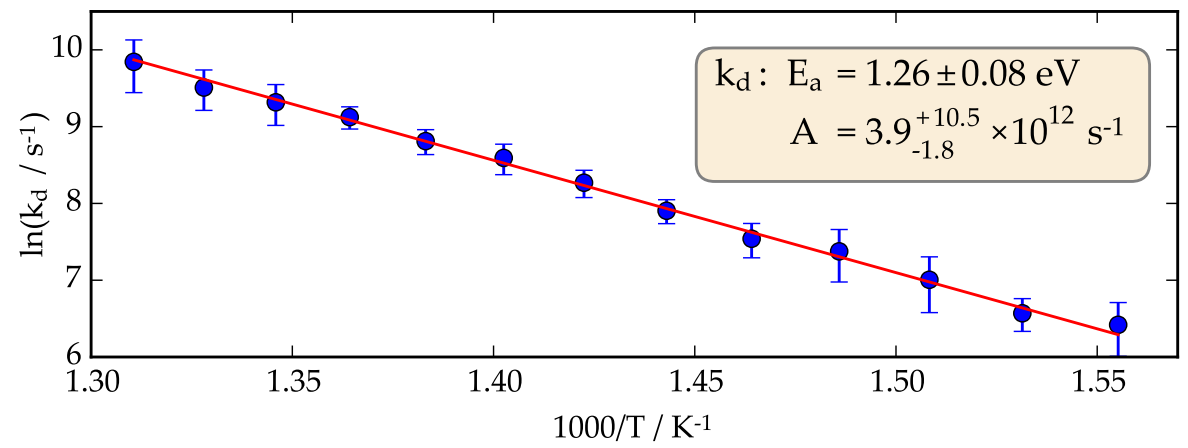

(c) Arrhenius analysis

Figure 5.5: Panel (a): Flux of $\mathrm{CO}$ desorbing from a $\mathrm{Pt}(332)$ surface. Kinetic traces for several surface temperatures and their respective fits are shown. The temperature of the traces increases from top to bottom. Panel (b): The kinetic trace at $683 \mathrm{~K}$, the fit function (black) and the different contributions to the fit (blue and lime green, dashed). Panel (c): Arrhenius plot. The result of the error-weighted fit is annotated in the box. 


\section{$5 \cdot 4$ DISCUSSION}

We compare the results measured on $\mathrm{Pt}(111)$ and $\mathrm{Pt}(332)$ to different experimental reports. The studies introduced below distinguish themselves by offering a clear assignment of the investigated processes. The reported rate coefficients are presented in table 5.1.

In a recent work Golibrzuch et al. obtained two rate coefficients from bi-exponential fits to the velocity-selected $\mathrm{CO}$ desorption flux. The fast desorption component was assumed to be desorption from $\mathrm{Pt}(111)$ terraces, while the slower component was assigned to stepassisted desorption.

Verheij and Comsa et al. investigated the trapping-desorption in a molecular beam relaxation spectroscopy (MBRS) experiment. In the same study they report thermal energy atom (helium) scattering (TEAS) results, which are expected to exclusively detect the desorption from terraces. Taking into account the good agreement of the different methods combined with the rather flat $\mathrm{Pt}(111)$ surface (step density less than $0.2 \%$ ), the authors conclude that the reported values "are representative of a defect free surface" [43].

Gdowski and Madix [44] measured the desorption of CO from $\mathrm{Pt}(111)$ in the presence of sulfur using Auger electron spectroscopy (AES), TPD and modulated beam techniques. Sulfur was used to block the steps and defects - the result can thus be considered the unbiased desorption from terraces.

An overview of the Arrhenius parameters from the reports listed above is shown in table 5.I. It is very difficult to tell how well the

Table 5.1: Arrhenius parameters.

\begin{tabular}{|c|c|c|}
\hline & $\begin{array}{c}\text { activation energy } \\
\mathrm{eV}\end{array}$ & $\begin{array}{l}\text { prefactor } \\
\mathrm{s}^{-1}\end{array}$ \\
\hline This work, $\operatorname{Pt}(111)$ & $1.28 \pm 0.02$ & $5.9_{-2.8}^{+5.4} \times 10^{13}$ \\
\hline Golibrzuch et al. [41] terraces & $1.27 \pm 0.07$ & $3.5_{-2.7}^{+7.2} \times 10^{13}$ \\
\hline Comsa et al. [43] terraces & 1.39 & $4.3 \times 10^{14}$ \\
\hline Gdwoski, Madix [44] terraces & 1.19 & $1 \times 10^{13}$ \\
\hline This work, $\mathrm{Pt}(332)$ & $1.26 \pm 0.08$ & $3.9_{-1.8}^{+10.5} \times 10^{12}$ \\
\hline Golibrzuch et al. [41] steps & $1.05 \pm 0.1$ & $1.9_{-1.0}^{+2.8} \times 10^{11}$ \\
\hline
\end{tabular}

different measurements agree with each other from the Arrhenius parameters alone. A direct comparison of the desorption rate coefficients for $\mathrm{Pt}(332)$ and $\mathrm{Pt}(111)$ with literature values is shown in figure 5.6. A comparison of the rate coefficients is preferable to a comparison of prefactors and activation energies characterizing the process. 


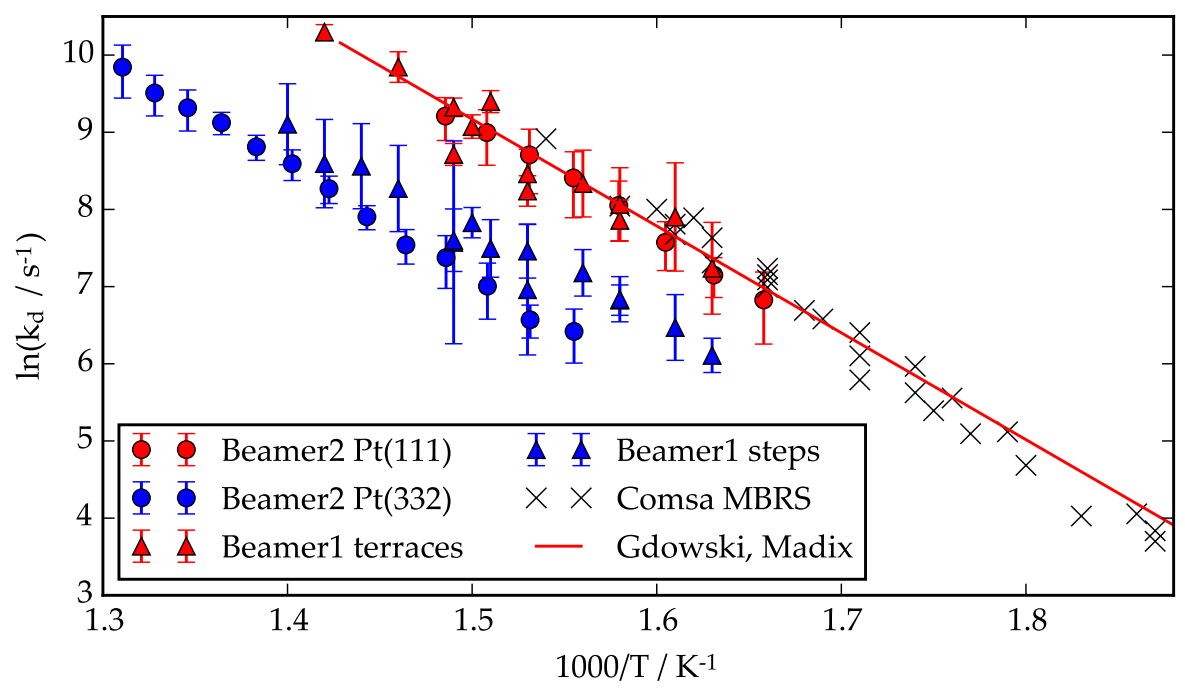

Figure 5.6: Overview of $\mathrm{CO}$ desorption rates: The desorption rates on $\operatorname{Pt}(111)$, shown as red dots, agree with the value assigned to desorption from terraces as measured by Golibrzuch et al. [41] (red triangles), Comsa et al. [43] (black cross) and Gdowski, Madix [44] (red line). The desorption from a $\mathrm{Pt}(332)$ surface (blue dots) agrees with the slow component of Golibrzuch et al. (blue triangle), which was assigned to desorption from steps.

The correlated error between $\mathrm{A}$ and $\mathrm{E}_{\mathrm{a}}$ can render such a comparison misleading.

Figure 5.6 shows that the values reported for desorption from $\mathrm{Pt}(111)$ terraces are in excellent agreement with the rate coefficients we measured on the $\operatorname{Pt}(111)$ crystal. The rate coefficient for indirect desorption from steps measured on the $\mathrm{Pt}$ (332) crystal are slightly lower than the ones measured by Golibrzuch et al. at the Beamer 1 experiment. According to the Serri-Cardillo-Tully model [42] the desorption rate coefficient depends on the equilibrium of $\mathrm{CO}$ on terrace and step sites and thus slows down with increasing step density. The $\mathrm{Pt}(332)$ crystal has a higher step density than the Pt(111) crystal and therefore we expect to see lower desorption rate coefficients. A detailed discussion of the model and further analysis based on transition state theory (TST) can be found in Dmitriy Borodin's master thesis [45].

\section{REFERENCES}

[38] C.T. Campbell, G. Ertl, H. Kuipers, and J. Segner. "A molecular beam investigation of the interactions of $\mathrm{CO}$ with a $\mathrm{Pt}(111)$ surface." In: Surface Science Letters 107.1 (May 1981), A222. DoI: 10. 1016/0167-2584 (81) 90554 - 5 (cit. on pp. 46, 62, 100).

[41] Kai Golibrzuch, Pranav R. Shirhatti, Jan Geweke, Jörn Werdecker, Alexander Kandratsenka, Daniel J. Auerbach, Alec M. Wodtke, and Christof Bartels. "CO Desorption from a Catalytic Surface: 
Elucidation of the Role of Steps by Velocity-Selected Residence Time Measurements." In: Journal of the American Chemical Society 137.4 (2015), pp. 1465-1475. DOI: $10.1021 /$ ja509530k (cit. on pp. 61, 62, 70, 71, 90).

[42] J. A. Serri, J. C. Tully, and M. J. Cardillo. "The influence of steps on the desorption kinetics of NO from Pt(111)." In: The Journal of Chemical Physics 79.3 (1983), pp. 1530-1540. Dor: 10. 1063/1. 445946 (cit. on pp. 67, 71).

[43] Laurens K. Verheij, Joachim Lux, A Brad Anton, Bene Poelsema, and George Comsa. "A molecular beam study of the interaction of CO molecules with a Pt (111) surface using pulse shape analysis." In: Surf. Sci. 182.3 (1987), pp. 390-410. DoI: 10. 1016/0039-6028 (87)90008-2 (cit. on pp. 70, 71).

[44] G. E. Gdowski and R. J. Madix. "The effect of sulfur on CO adsorption/desorption on $\mathrm{Pt}(\mathrm{S})-[9(111) \times(100)]$." In: Surface Science 115.3 (Mar. 1982), pp. 524-540. DOI: 10 . 1016 / 0039 6028 (82) 90385- 5 (cit. on pp. 70, 71).

[45] Dmitriy Borodin. "Probing Reactions at Surfaces using Ion Imaging - CO Oxidation at Atomically Flat and Stepped Surfaces of Platinum and Palladium." Master Thesis. Georg-AugustUniversity Goettingen, 2017 (cit. on pp. 71, 142). 


\subsection{EXPERIMENTAL SETUP}

The experimental setup has been described in chapter 2 . The necessary changes to the setup and the experimental procedures in order to measure kinetics of bimolecular reactions are described below.

- Main beam: The main molecular beam was produced by a homebuilt PZT valve, which generated supersonic beam pulses along the surface normal. It was triggered by a $70 \mu \mathrm{s}, 1200 \mathrm{~V}$ pulse to achieve a rather long nozzle opening time. It was backed by 7 bar of neat $\mathrm{O}_{2}(99.999 \%$ pure), generating pulses with a flux on the surface of approximately $1.05 \times 10^{11}$ molecules/pulse. This beam was used to dose the surface with $\mathrm{O}_{2}$ to produce adsorbed oxygen atoms, $\mathrm{O}_{\mathrm{a}}$, by dissociative adsorption. The beam diameter on the surface was about $3 \mathrm{~mm}$.

- Side beam: The side molecular beam was produced by a second home-built PZT valve, which generated supersonic beam pulses introduced at $22^{\circ}$ with respect to the surface normal. It was triggered by a $25 \mu \mathrm{s}, 1000 \mathrm{~V}$ pulse to achieve a short nozzle opening time. It was backed by 7 bar of a $5 \%$ mix of $\mathrm{CO}$ in $\mathrm{He}$, generating narrow pulses with a FWHM of about 25-30 $\mu$ s with a flux on the surface of approximately $2.2 \times 10^{10}$ molecules/pulse. For a sticking probability of 0.8 , this equals a dose $\theta_{\text {dose }}$ of $1.47 \times 10^{-5}$ monolayer (ML), where $1 \mathrm{ML}$ is the number of $\mathrm{Pt}$ atoms per square centimeter, i.e. $1.5 \times 10^{15} / \mathrm{cm}^{2}$. The diameter on the surface was also about $3 \mathrm{~mm}$. The beams were aligned to overlap completely on the surface.

- Molecular beam trigger: The $\mathrm{O}_{2}$ beam was triggered at least $900 \mu$ s before the laser pulse to ensure full accommodation and dissociation of the $\mathrm{O}_{2}$-molecules. In experiments where the $\mathrm{CO}$ and the $\mathrm{O}_{2}$ beam had different repetition rates, the $\mathrm{O}_{2}$ beam was triggered asynchronously. The $\mathrm{CO}$ beam was used to initiate the reaction. Therefore the beam-laser delay in this thesis is always with respect to the $\mathrm{CO}$ beam.

- Steady-state conditions: Before recording data the respective repetition rates for the molecular beams were set and the beams were run until the production of $\mathrm{CO}_{2}$ per pulse had stabilized. When turning off the $\mathrm{O}_{2}$-beam while keeping the CO-beam on, the $\mathrm{CO}_{2}$ signal could be seen for several thousand pulses before

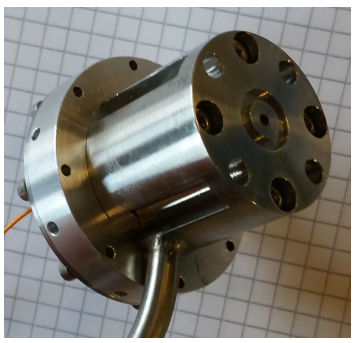

For more information on the timing of the two molecular beams see Appendix C.2.

The repetition rates were varied between 10 and $333 \mathrm{~Hz}$. 
it decayed. From this behaviour we conclude that the change in concentration of O-atoms is very small per pulse of $\mathrm{CO}$ and that the $\mathrm{O}$-atom concentration is replenished by pulses of $\mathrm{O}_{2}$ in between - a steady-state $\mathrm{O}$-atom concentration is established when running both beams.

- Laser system: The $\mathrm{CO}_{2}$ reaction products were ionized using strong-field multi-photon ionization (MPI) with a femtosecond laser ( $1-5 \mathrm{~mJ} /$ pulse, $<100 \mathrm{fs}, 800 \mathrm{~nm}$ ), focused by a $200 \mathrm{~mm}$ focal length lens $\left(3 \mathrm{~mm} \times 0.03 \mathrm{~mm}^{2}\right.$ detection volume). For measurements of the angular distribution, the position of the laser focus was scanned by moving the lens and therefore the lens focus parallel to the surface. For the kinetics measurements, the laser position was held fixed and probed reaction products at the surface normal.

- Detector: The setup of the ion optics and the MCP/phosphor stack is described in detail in section 4.1.2. By pulsing the front face of the MCP, it acted as a mass gate and selectively detected $\mathrm{CO}_{2}$ ions. While the laser slicing already selected ions with little velocity along $\mathrm{z}$ (towards the detector), the selection was not perfect due to the spatial extent of the surface. A narrow gate of about 100 to 200 ns was used to further slice the resulting ion cloud to select ions with zero velocity along $\mathrm{z}$.

- Reaction conditions: The CO oxidation was studied at temperatures between $563 \mathrm{~K}$ and $723 \mathrm{~K}$. However, temperatures above $623 \mathrm{~K}$ gave rise to kinetic rates that were too fast to be measured within the time-resolution of the experiment. Seven temperatures from $563-623 \mathrm{~K}$ were used for this analysis. For each temperature, different beam fluxes were used to obtain different oxygen coverages. The time-averaged beam flux depends linearly on the beam repetition rate, therefore the ratio between the two fluxes was varied by changing the repetition rate ratio (RRR) of the two pulsed valves. An RRR of 1 is equivalent to both beams running at $100 \mathrm{~Hz}$, which yields a time-averaged $\mathrm{O}_{2}$ flux of $1.1 \times 10^{13}$ molecules $/ \mathrm{cm}^{2} \mathrm{~s}$ and a time-averaged $\mathrm{CO}$ flux of $2.2 \times 10^{12}$ molecules $/ \mathrm{cm}^{2} \mathrm{~s}$. An $\mathrm{O}_{2}$ repetition rate of $200 \mathrm{~Hz}$ and a $\mathrm{CO}$ repetition rate of $100 \mathrm{~Hz}$ would lead to a doubled $\mathrm{O}_{2}$ flux and an RRR of 2.

6.2 ION IMAGES OF $\mathrm{CO}_{2}$ AT DIFFERENT REACTION CONDITIONS

A typical ion image obtained using the angular-scan setup described above is shown in figure 6.1a. Because the fs-laser ionization volume was very small, the focus of the laser had to be scanned parallel to the surface to record angular distribution of the $\mathrm{CO}_{2}$ molecules. This is indicated by the vertical cyan line in the figure. The further on the 
right of the laser the ions are detected, the faster they were moving when they passed the laser. Therefore the $\mathrm{CO}_{2}$ ions next to the laser have a lower velocity than the ions far away from the laser.

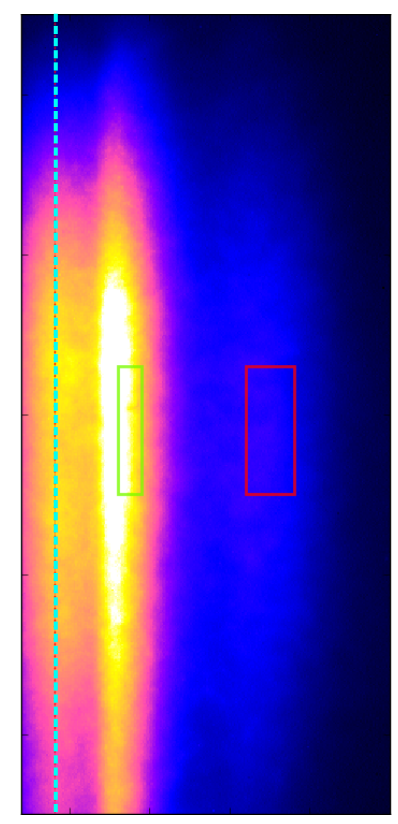

(a) raw density image

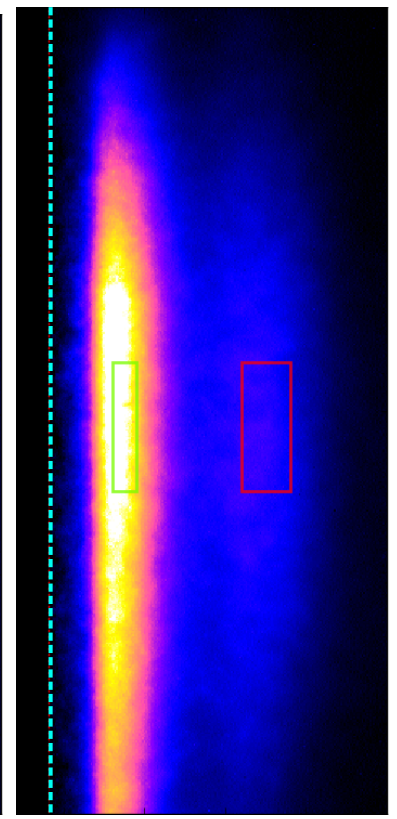

(b) background-corrected density image

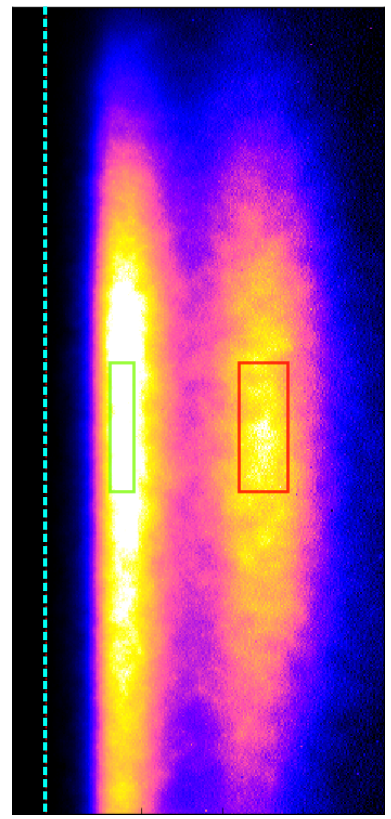

(c) background-corrected flux image

Figure 6.1: Sum ion images of $\mathrm{CO}_{2}$ recorded over the full accessible angular range at $723 \mathrm{~K}$. (a) Raw density image. The laser focus was scanned along the cyan, dashed line to record the angular distribution. (b) The $\mathrm{CO}_{2}$ background signal was removed by mirroring the image part left of the laser and subtracting it from the right side. (c) The background-corrected density image in panel $\mathrm{b}$ was transformed into a flux image.

Three $\mathrm{CO}_{2}$ components with different velocities can be identified in panel (a). Background $\mathrm{CO}_{2}$ in the machine leads to a background signal centered around the laser position. On the right of the laser there are two components, a slow $\mathrm{CO}_{2}$ species (bright yellow, i.e. high intensity), and a fast $\mathrm{CO}_{2}$ species (dark blue, low intensity), which are both moving away from the surface.

By mirroring the image part on the left of the laser and subtracting it from the right part we can remove any background contribution. This is important for both dynamical analysis (speed and angular distributions) and for kinetic traces, because the $\mathrm{CO}_{2}$ background, or more specifically the $\mathrm{CO}_{2}$ background pressure between the lower repeller grid and the upper grid, is modulated by the $\mathrm{CO}_{2}$ production after each $\mathrm{CO}$ pulse. In this case, the background can be distinguished quite well from both other components, because even the slower $\mathrm{CO}_{2}$ is fast compared to the background molecules. The resulting, background-corrected density ion image is shown in figure 6.1b.

A cropped image is shown, the actual image extends further to all sides.

This can become a problem when looking at thermal processes, e.g. desorption, at low temperatures! 
See section 4.2 .3 for density-to-flux.
This density image can be transformed into a flux image by multiplying each pixel with its respective velocity. The resulting flux image is shown in figure 6.1c. In this representation a bimodal distribution in both speed and angle, with the faster component peaking sharply around the surface normal, becomes apparent.

A set of flux sum ion images is shown in figure 6.2. The figure shows one temperature per row and different RRRs per temperature, as noted in the caption below each ion image. At any given temperature, the hyperthermal channel increases in strength relative to the thermal channel with increasing $\mathrm{O}$-atom coverage. When going to higher temperatures, the hyperthermal channel abundance increases strongly.

The images at RRR 10 on the right side appear slightly more pixelated than the other ones. This is due to the lower number of ion imaging cycles - To achieve an RRR of 10 the $\mathrm{O}_{2}$-beam was triggered with $333 \mathrm{~Hz}$ and the CO-beam was triggered with $33 \mathrm{~Hz}$. Since the ion extraction and the detector unit are triggered concomitant with the CO-beam, these were triggered less often than e.g. when running both beams at $100 \mathrm{~Hz}$. For a similar acquisition time, this leads to less signal causing each single ion spot to stand out more clearly. 


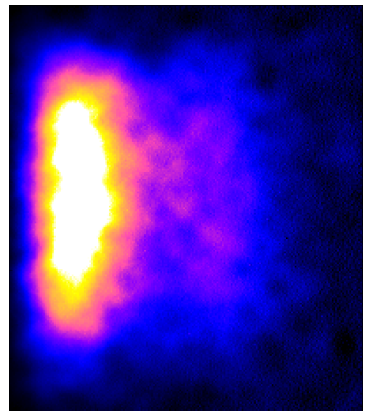

(a) $563 \mathrm{~K}, \mathrm{RRR} 1$

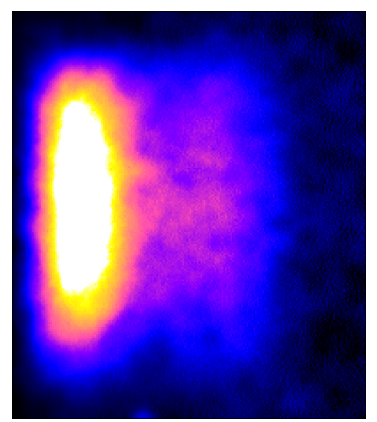

(b) $583 \mathrm{~K}, \mathrm{RRR}_{1}$

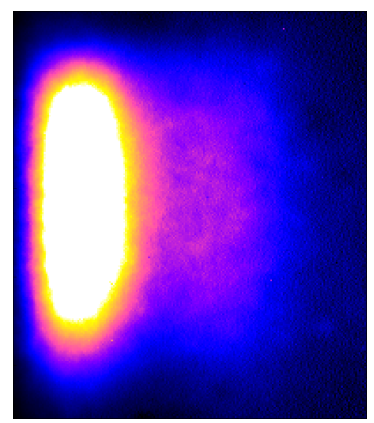

(c) $603 \mathrm{~K}, \mathrm{RRR} 1$

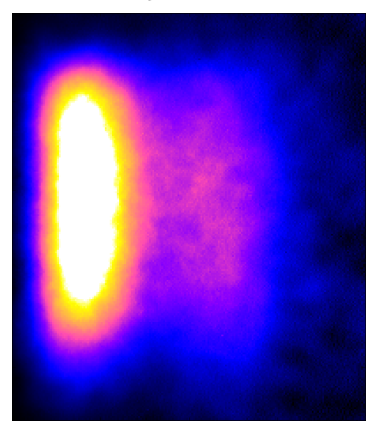

(d) $623 \mathrm{~K}, \mathrm{RRR} 1$

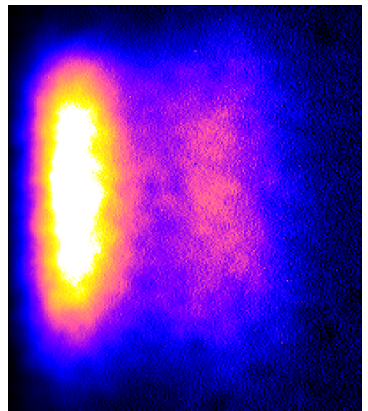

(e) $563 \mathrm{~K}, \mathrm{RRR} 5$

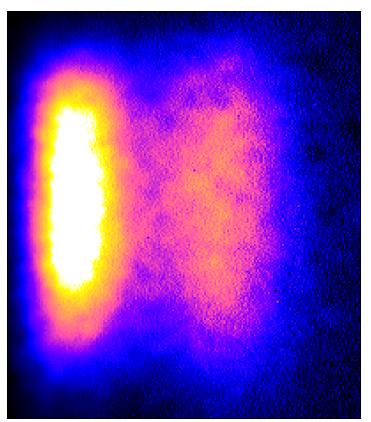

(f) $583 \mathrm{~K}, \mathrm{RRR} 5$

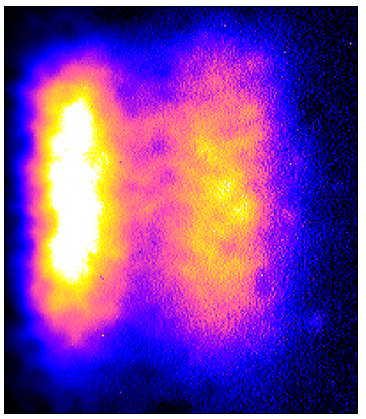

(g) $603 \mathrm{~K}, \mathrm{RRR} 5$

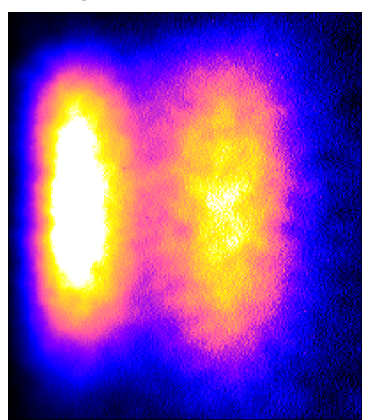

(h) $623 \mathrm{~K}, \mathrm{RRR} 5$

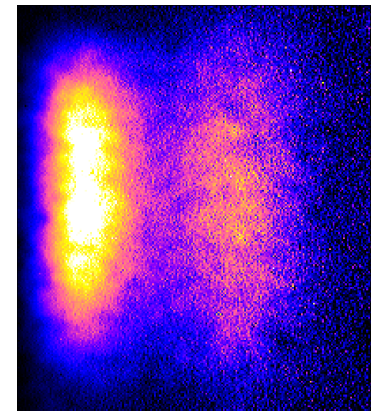

(i) $563 \mathrm{~K}, \mathrm{RRR} 10$

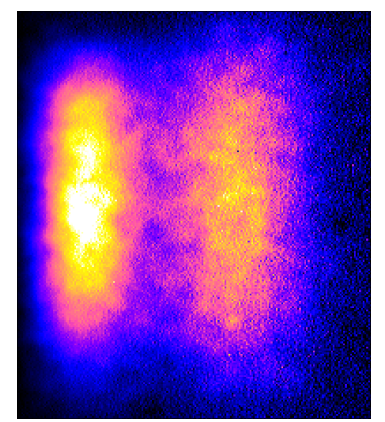

(j) $583 \mathrm{~K}$, RRR 10

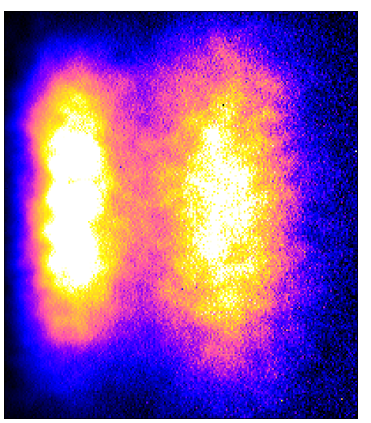

(k) $603 \mathrm{~K}, \mathrm{RRR} 10$

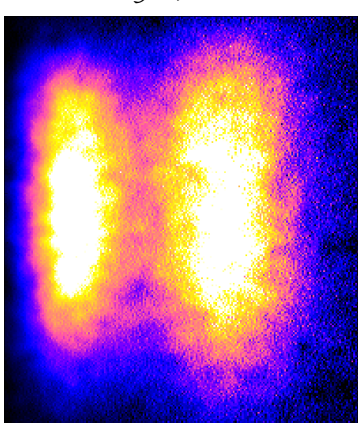

(1) $623 \mathrm{~K}, \mathrm{RRR} 10$

Figure 6.2: Overview of flux sum ion images vs reaction conditions: The RRR is increased from left to right, the surface temperature is increased from top to bottom. The maximum intensity has been rescaled for each image to be able to compare the relative strength of thermal and hyperthermal $\mathrm{CO}_{2}$. 


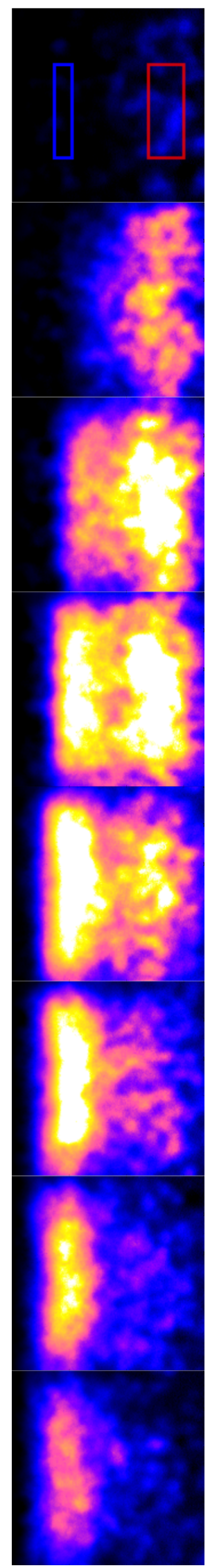

(a) selected ion images

\subsection{KINETIC TRACES FROM ION IMAGES}

A series of selected images depicting the temporal evolution of the $\mathrm{CO}_{2}$ flux signal as a function of beam-laser delay are shown in figure $6.3 \mathrm{a}$ on the side. The laser position was precisely at the left border of each image, the velocity increases from left to right. From top to bottom the beam-laser delay of the respective image increases. At early delays, only a few microseconds after the $\mathrm{CO}$ beam pulse arrives at the surface, a few fast $\mathrm{CO}_{2}$ ions can be seen on the right of the top picture. $\mathrm{CO}_{2}$ molecules with high velocities need less time to travel from the surface to the ionizing laser spot, which is the reason why the slower $\mathrm{CO}_{2}$ only begin to appear in the third ion image from the top. By integrating the intensity inside the two ROIs, shown as blue and red boxes in the top ion image, for each ion image and plotting it against the respective delay, we obtain the kinetic profile shown in figure 6.3b. Points from the ion images in the side panel are

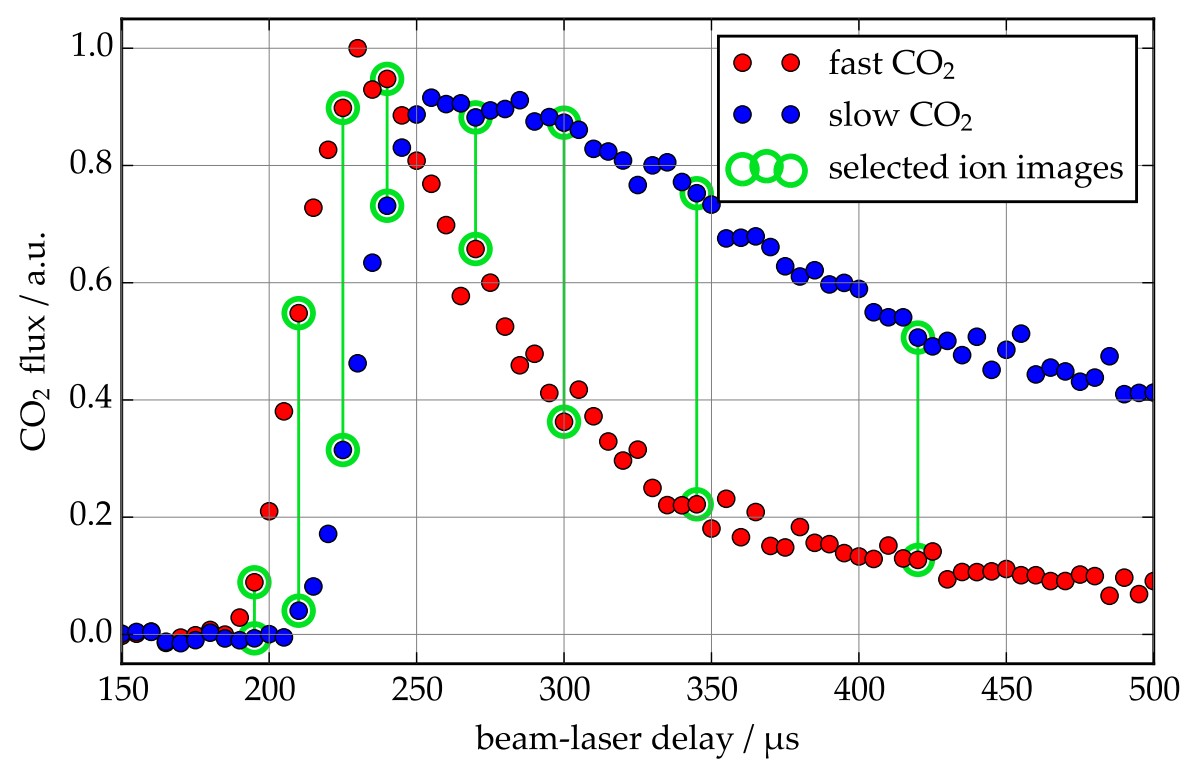

(b) Extracted kinetic profile

Figure 6.3: (a) A selected series of flux ion images, with beam-laser delay increasing from top to bottom, is shown on the side. The ROIs used for integration are shown in the top image. The scan was recorded at $723 \mathrm{~K}$ and an RRR of 1 . (b) The raw kinetic profile obtained by integrating the signal in the ROIs for each beam-laser delay. The points in the graph corresponding to the ion image series from panel (a) are highlighted by green circles. Vertical green links are used to connect points from the same ion image.

marked with green circles and connecting lines. From inspection of the first two marked pairs (i.e., the signal from the top two ion images) one sees that the fast $\mathrm{CO}_{2}$ molecules arrive at the laser position earlier than the slower $\mathrm{CO}_{2}$ molecules. Between the fourth and the fifth ion image the intensity ratio reverses and the slow $\mathrm{CO}_{2}$ channel 
reaches its maximum, while the fast $\mathrm{CO}_{2}$ flux is already decaying. The kinetic profile across the whole scan range is shown in figure 6.4. Panel (a) shows the kinetic profile extracted from density ion images, while flux ion images were used for the graph in panel (b). The slow

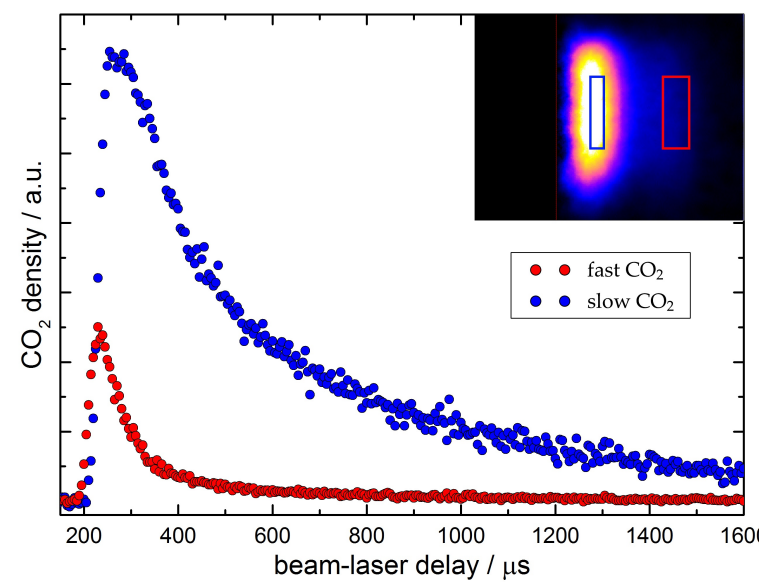

(a) raw kinetic profile from density images

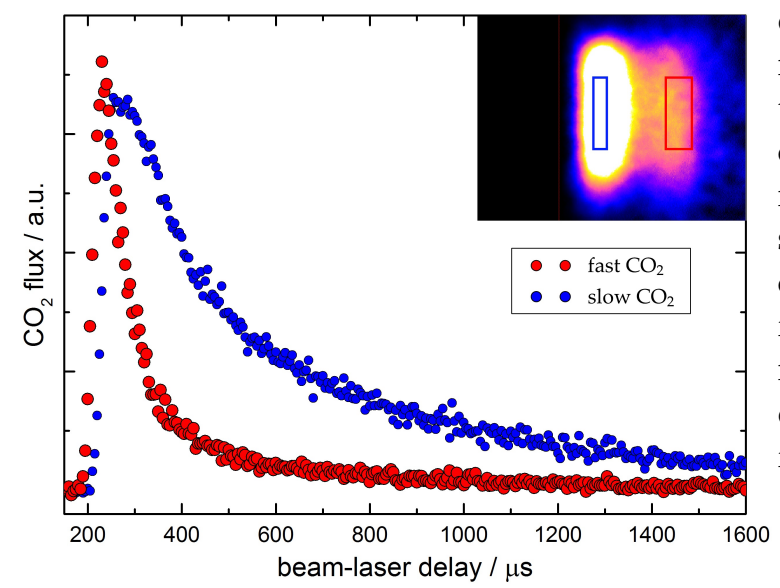

(b) raw kinetic profile from flux images
Figure 6.4: Raw kinetic profile obtained from density images (top) and from flux images (bottom). A sum ion image of the underlying scan is shown as an inset. The integration region is indicated by a blue and a red box. The slow component clearly dominates when density ion images are used for the integration, as one can predict from the ion image shown as an inset. After a density-to-flux conversion is applied, the fast component increases in relative strength. Reproduced with permission from Ref. [46].

$\mathrm{CO}_{2}$ signal (blue dots) in panel (a) has a higher intensity than the fast $\mathrm{CO}_{2}$ and decays on a millisecond timescale. The fast $\mathrm{CO}_{2}$ signal (red dots) in panel (a) is much weaker than the slow $\mathrm{CO}_{2}$ and decays on a faster timescale on the order of $100 \mu \mathrm{s}$. While the shape and the timing is preserved in panel (b), the relative signal intensity of the fast component increases when the kinetic profile is calculated from flux images. Due to the higher velocity, the pixels comprising the fast $\mathrm{CO}_{2}$ are multiplied by larger numbers, thus increasing their relative signal strength. Now that the two profiles have a similar amplitude, another detail is revealed - the rising edge of the fast $\mathrm{CO}_{2}$ is shifted to earlier times by about $20-40 \mu \mathrm{s}$.

Before we can compare the relative fluxes, there are two additional factors which need to be taken into account: The flux shown in figure 6.4 has been integrated over only a small, velocity-selected region of the ion image. One must correct for the relative widths of the

Keep in mind that we are not artificially increasing the intensity of the fast channel but merely correcting for the lower ionization probability. 
The process of calculating a speed distribution is explained in detail in section 4.2 .5 overall speed and angular distribution in order to arrive at the total relative flux in the two distinct channels.

First, the integration regions (ROIs) do not cover the full speed range. Moreover, we actually extract the mean signal in an integration region to be able to compare signals from differently sized regions more easily. In order to correct for this, we use the flux sum ion image to calculate the speed distribution shown in figure 6.5. As in

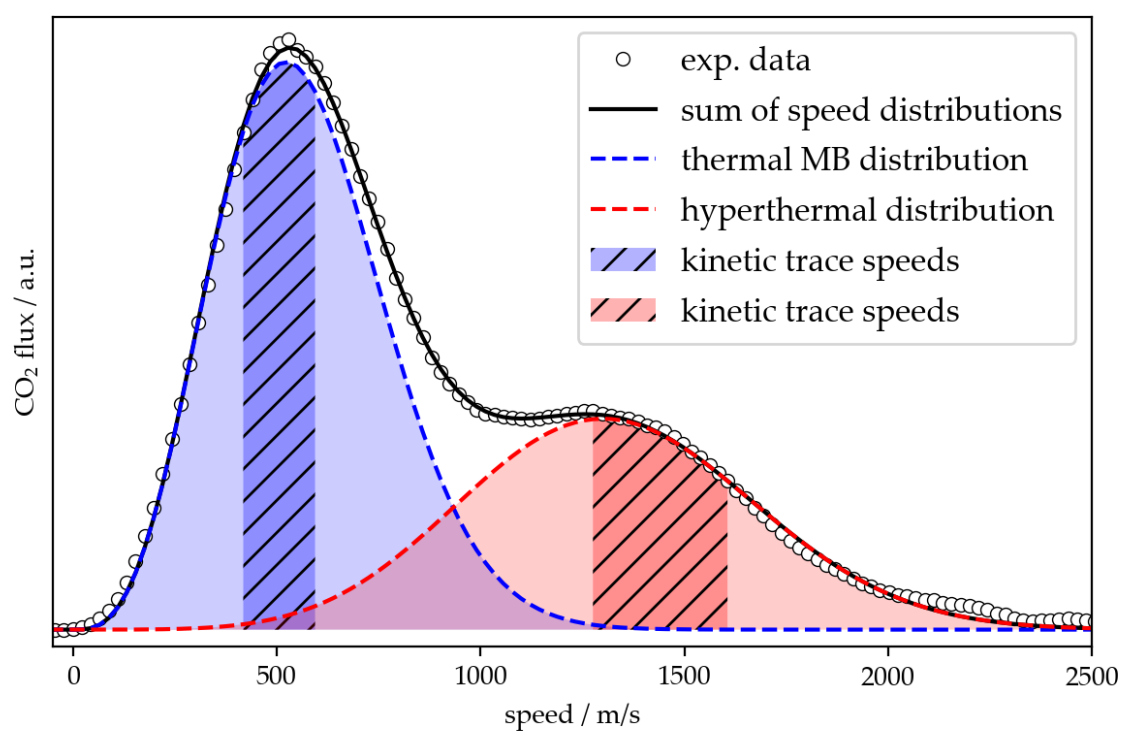

Figure 6.5: Speed distribution of $\mathrm{CO}_{2}$ calculated from a flux sum ion image. The speed distribution confirms the bimodality seen in the ion images above. The two components are fitted with a MB distribution and a hyperthermal distribution, see text. Reproduced with permission from Ref. [46].

the ion image, the bimodality in speed is obvious when looking at the speed distribution. The slow component was fitted with a MaxwellBoltzmann (MB) distribution (dashed, blue), described by

$$
f(v)=A v^{3} \exp \left(-\frac{m_{\mathrm{CO}_{2}} v^{2}}{2 k_{\mathrm{B}} T}\right)
$$

We sometimes refer to the hyperthermal distribution as "flowing $M B$ distribution", which is not entirely correct. Any distribution with an energy offset is by definition nonMaxwell-Boltzmann.
The fit suggests a slightly sub-thermal temperature of $483 \mathrm{~K}$ compared to the surface temperature of $613 \mathrm{~K}$. The fast component was best fit with a hyperthermal distribution [40] (dashed, red) of the form

$$
f(v)=A v^{3} \exp \left(-\frac{m_{\mathrm{CO}_{2}}\left(v-v_{\text {shift }}\right)^{2}}{2 k_{\mathrm{B}} T}\right),
$$

where $v_{\text {shift }}$ is the extra velocity of the hyperthermal component. The fit results $\left(894 \mathrm{~K}, \mathrm{~V}_{\text {shift }}=910 \mathrm{~m} \mathrm{~s}^{-1} \approx 185 \mathrm{meV}\right)$ agree reasonably well with previously reported values $[47,48]$. The slow component 
is henceforth denoted thermal (MB) and the fast component is denoted hyperthermal (HT) to avoid ambiguities, because "fast channel" could refer to a channel with fast velocities or a fast decay.

The kinetic profiles have been calculated using mean signal in the velocity range indicated by the hatched areas. The total flux into either channel in the kinetic profiles should however reflect the area under the speed distributions $A_{\mathrm{MB}}$ and $A_{\mathrm{HT}}$, as indicated by the blue and red shaded area, respectively. We therefore determine the flux in the raw kinetic profile by calculating the area under the curve (Flux$\mathrm{MB}$ and Flux-HT) and then multiply the kinetic profile of the thermal component by a factor $\mathrm{c}$ as described by

$$
\frac{A_{\mathrm{MB}}(\text { blue area })}{A_{\mathrm{HT}}(\text { red area })}=\frac{\text { Flux }-\mathrm{MB} \times \mathrm{c}}{\text { Flux }-\mathrm{HT}} .
$$

In a second step, we correct for the different angular distributions. An angular distribution is shown in figure 6.6 next to the ion image
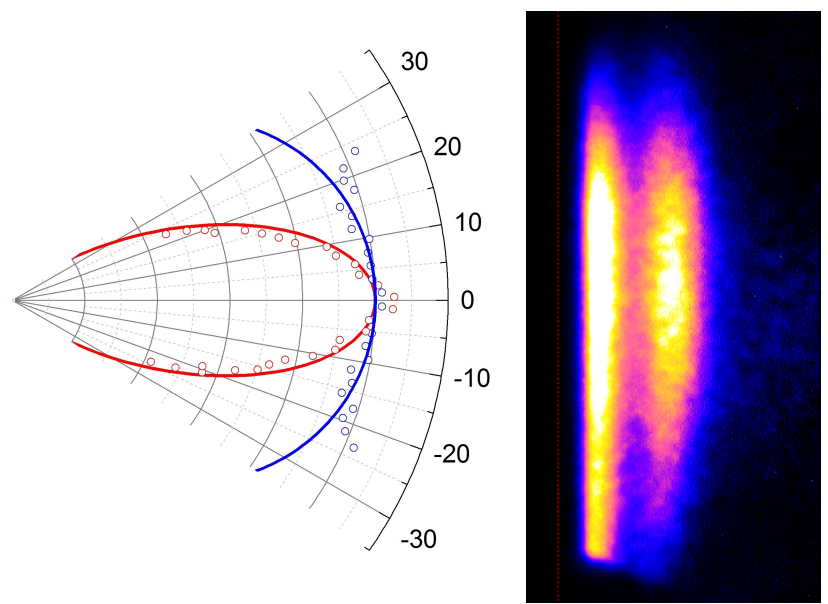

Figure 6.6: Angular distribution extracted from the ion image on the right. The origin of the angular distribution coincides with the surface coordinates. The thermal $\mathrm{CO}_{2}$ is compared to a $\cos (\vartheta)$ distribution (blue line) and the hyperthermal component is compared to $\cos ^{8}(\vartheta)$ distribution (red line). Reproduced with permission from Ref. [46].

from which it was extracted. The thermal $\mathrm{CO}_{2}$ (blue circles) is compared to a cosine distribution, the hyperthermal $\mathrm{CO}_{2}$ (red circles) is compared to $a \cos ^{8}(\vartheta)$ distribution (red line). This bimodality has been observed previously and the $\cos (\vartheta)$ and $\cos ^{8}(\vartheta)$ distributions we compare to are values used in earlier reports, such as ref [47-50].

Up until now the kinetic profiles have been scaled to the velocity distribution along a sector subtending $\pm 3^{\circ}$ around the surface normal. These $6^{\circ}$ contain a different fraction of the total $\mathrm{CO}_{2}$ flux depending on the sharpness of the angular distribution. We therefore need to A more detailed analysis of the dynamical information is presented in section 8.1.1 and 8.1.2. scale the kinetic profile by a factor correcting for the different angular 
The speed distribution was extracted by integrating along $a$ sector subtending $6^{\circ}$ around the surface normal, as introduced in section 4.2.5. distributions of the signal to obtain the total branching ratio between the two channels.

$$
\frac{\int_{0}^{3^{\circ}} \cos ^{8} \vartheta \sin \vartheta d \vartheta}{\int_{0}^{90^{\circ}} \cos ^{8} \vartheta \sin \vartheta d \vartheta} / \frac{\int_{0}^{3^{\circ}} \cos \vartheta \sin \vartheta d \vartheta}{\int_{0}^{90^{\circ}} \cos \vartheta \sin \vartheta d \vartheta}=4.7
$$

The fraction of $\mathrm{CO}_{2}$ included within the angular range that was used to extract the speed distribution in fig 6.5 is 4.7 times higher for the hyperthermal channel. Multiplication of the thermal channel by 4.7 yields the total branching ratio. The resulting kinetic profiles are shown in figure 6.7 .

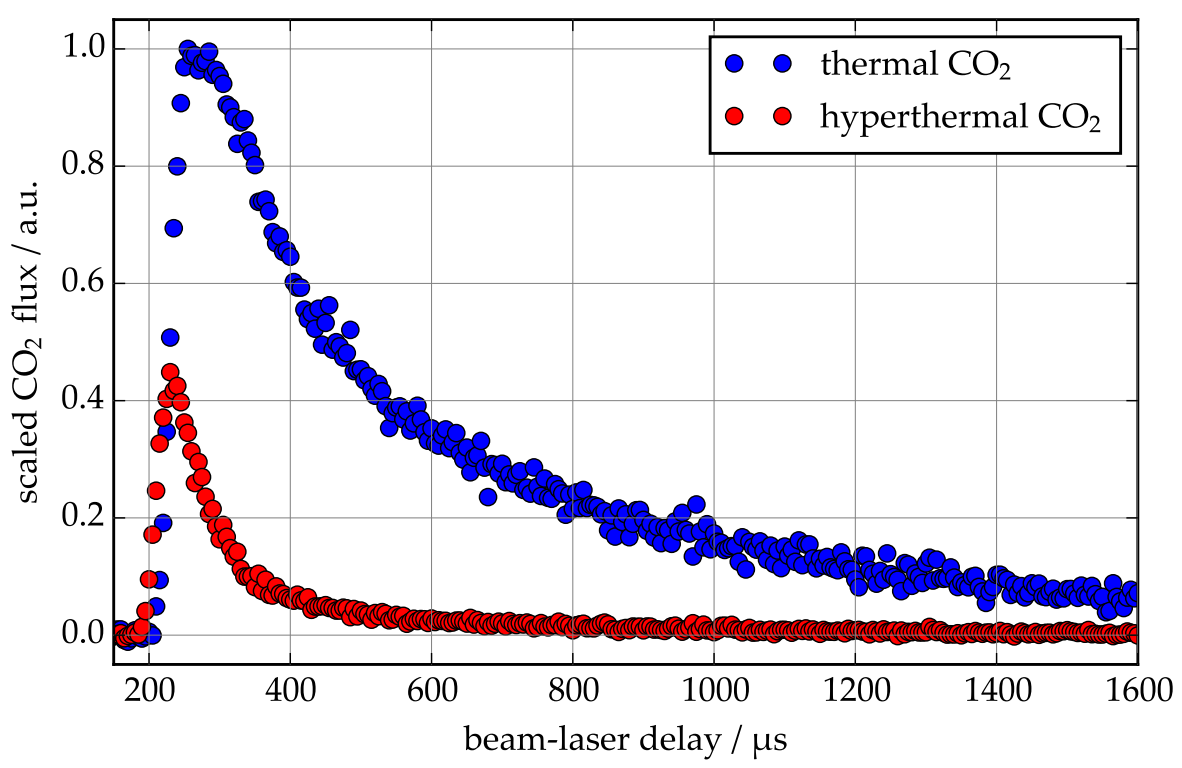

Figure 6.7: Scaled kinetic profile: The kinetic profile was scaled to reflect the true branching ratio between the two channels. Reproduced with permission from Ref. [46].

It is now clear that the thermal $\mathrm{CO}_{2}$ channel has a much higher total flux under the conditions of this scan. The flux ion image suggested that the branching ratio is only slightly in favor of the thermal channel. After introducing the angular distribution correction factor of 4.7 , the dominant channel becomes far more pronounced.

As a final step, the $\mathrm{x}$-axis of the figure must be converted from beam-laser delay into reaction time. The process is shown schematically in figure 6.8. The time at which the incident $\mathrm{CO}$ beam passes the laser is known from the measurement of the incident beam profile as shown in panel (b). The same scan also contains information on the speed distribution of the incoming beam, typically a narrow velocity range around $1500 \mathrm{~m} \mathrm{~s}^{-1}$. The distance between surface and laser focus along $x$ can be measured in the lab and was $19 \mathrm{~mm}$ for the ion images above. The incident beam is aligned $22^{\circ}$ from the surface nor- 


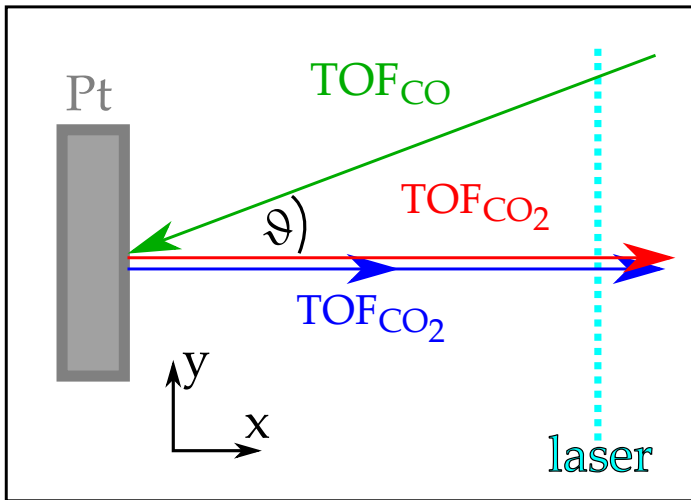

(a) geometry sketch

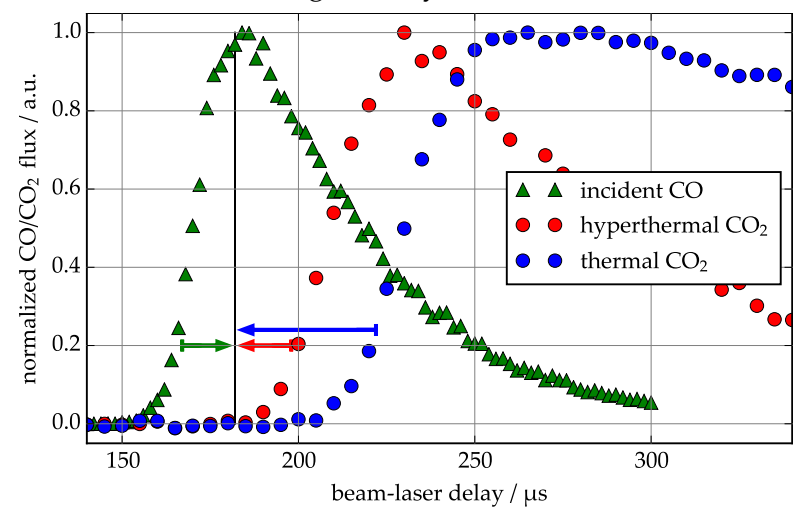

(b) resulting TOF shifts
Figure 6.8: (a) geometry sketch showing the travel distances for incident $\mathrm{CO}$ and $\mathrm{CO}_{2}$ products. The corresponding TOF is used to shift the kinetic profiles. (b) incident $\mathrm{CO}$ flux and product $\mathrm{CO}_{2}$ flux vs beam-laser delay. All data was normalized in order to compare the rising edge. After correcting for TOF shifts, the rising edge of product $\mathrm{CO}_{2}$ coincides with the arrival time of the incident $\mathrm{CO}$ beam, as indicated by the black vertical line.

mal, which allows the distance along the green arrow to be calculated as

$$
d_{\text {CO-path }}=\frac{d_{\text {surf-laser }}}{\cos \vartheta}=\frac{19 \mathrm{~mm}}{\cos (22 \pi / 180)}=20.5 \mathrm{~mm} \text {. }
$$

Knowing the velocity and the distance, we can calculate the time of flight of the CO pulse from the laser to the surface to be $13 \mu \mathrm{s}$. We therefore shift the incident beam profile by $13 \mu \mathrm{s}$, as indicated by the green arrow in panel (b), and define this time of arrival as $t=0 \mu \mathrm{s}$. The time of arrival is indicated by the vertical black line. The velocities used to calculate the kinetic profiles are shown as hatched areas in figure 6.5 , i.e. $500 \mathrm{~m} \mathrm{~s}^{-1}$ for the thermal $\mathrm{CO}_{2}$ and $1400 \mathrm{~m} \mathrm{~s}^{-1}$ for the hyperthermal $\mathrm{CO}_{2}$. For a flight distance of $19 \mathrm{~mm}$ this yields flight times of $38 \mu$ s and $14 \mu$ s, respectively. By shifting all kinetic profiles by the respective TOF and defining the rising edge of the incoming beam to be at $t=0 \mu \mathrm{s}$, the $\mathrm{x}$-axis is transformed from beam-laser delay into reaction time. The resulting kinetic trace is shown in figure 6.9

The rising edges of both the incident $\mathrm{CO}$ beam and the $\mathrm{CO}_{2}$ products overlap nicely, suggesting the TOF shifts were calculated correctly. One detail to be aware of is the arrival time distribution of $\mathrm{CO}$ on the surface. Since the incident beam is not infinitely narrow, the observed signal is a convolution of the incident beam profile and the kinetics governing the surface reaction. Consequently, the analysis has to 
The LH mechanism will be introduced in section 6.4
Decay time is chosen instead of reaction time to include other processes depleting $\mathrm{CO}$ on the surface.

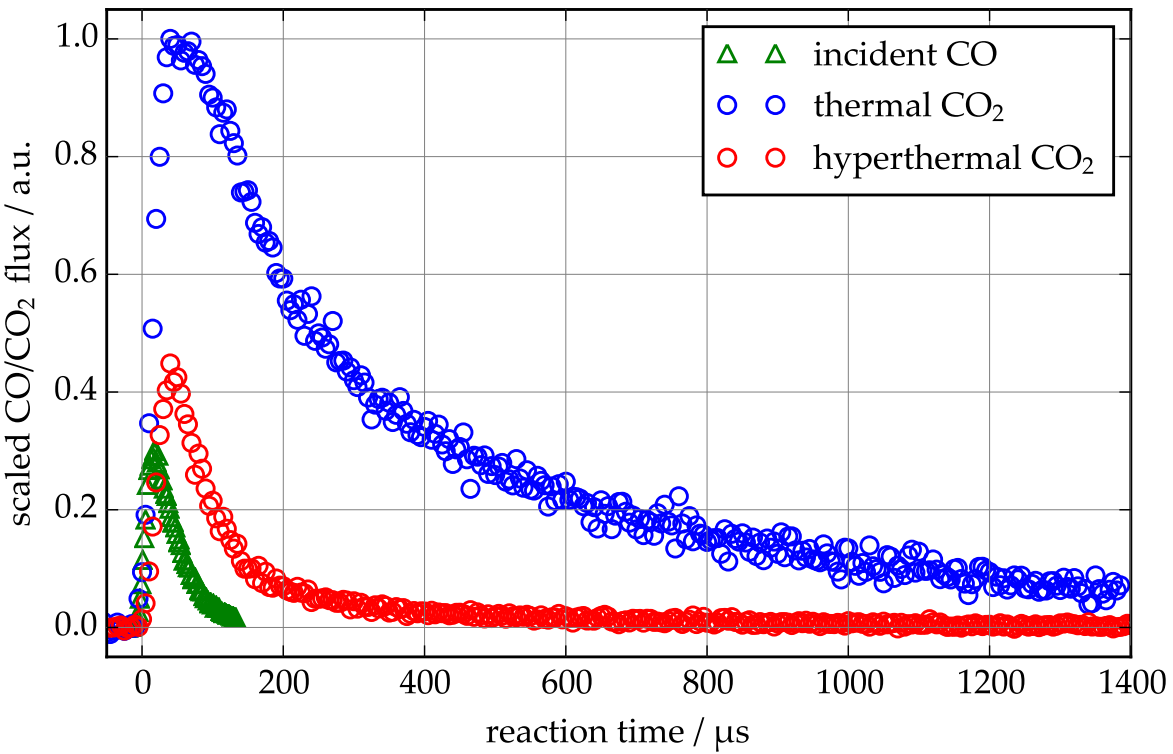

Figure 6.9: Kinetic trace: The $\mathrm{x}$-axis has been transformed into reaction time of $\mathrm{CO}$ on the surface. The underlying scan was recorded at $350^{\circ} \mathrm{C}$ with both molecular beams running at a repetition rate of $100 \mathrm{~Hz}$, i.e. a time-averaged $\mathrm{O}_{2}$ flux of $1.1 \times 10^{13}$ molecules $/ \mathrm{cm}^{2} \mathrm{~s}$ and a CO flux of $2.2 \times 10^{12}$ molecules $/ \mathrm{cm}^{2} \mathrm{~s}$. The incident beam is scaled arbitrarily to compare the rising edges.

deconvolute these contributions - which works best for narrow incoming beams and slow kinetics.

If the width of the incoming beam pulse is small enough to be neglected, the kinetic trace is the flux of product molecules leaving the surface as a function of time on the surface, i.e. the reaction time. A measurement of the relative flux of product molecules is a measurement of the relative reaction rate as a function of time after the $\mathrm{CO}$ pulse. If the rate is proportional to the concentration of the reactant on the surface, as can be assumed for a first order or a pseudo-first order Langmuir-Hinshelwood (LH) reaction, the kinetic trace is a measurement of the concentration profile of the reactant. The reaction rate can then be determined without knowledge of the absolute fluxes of incident beams and product molecules.

Note that the kinetic trace has been plotted using open circles. This plotstyle will henceforth be used for data that was treated following the procedure described above. Open circles signify data that was TOF-shifted and intensity-scaled.

Figure 6.2 showed the flux sum ion images of experimental measurements at different reaction conditions. The kinetic traces obtained from the same measurements are shown in figure 6.10. The RRR and temperature for each scan is given as an inset. A few notable observation concerning the shape of the kinetic traces are listed below:

- For a given temperature, the decay times for both channels decrease with increasing RRR (from left to right). 


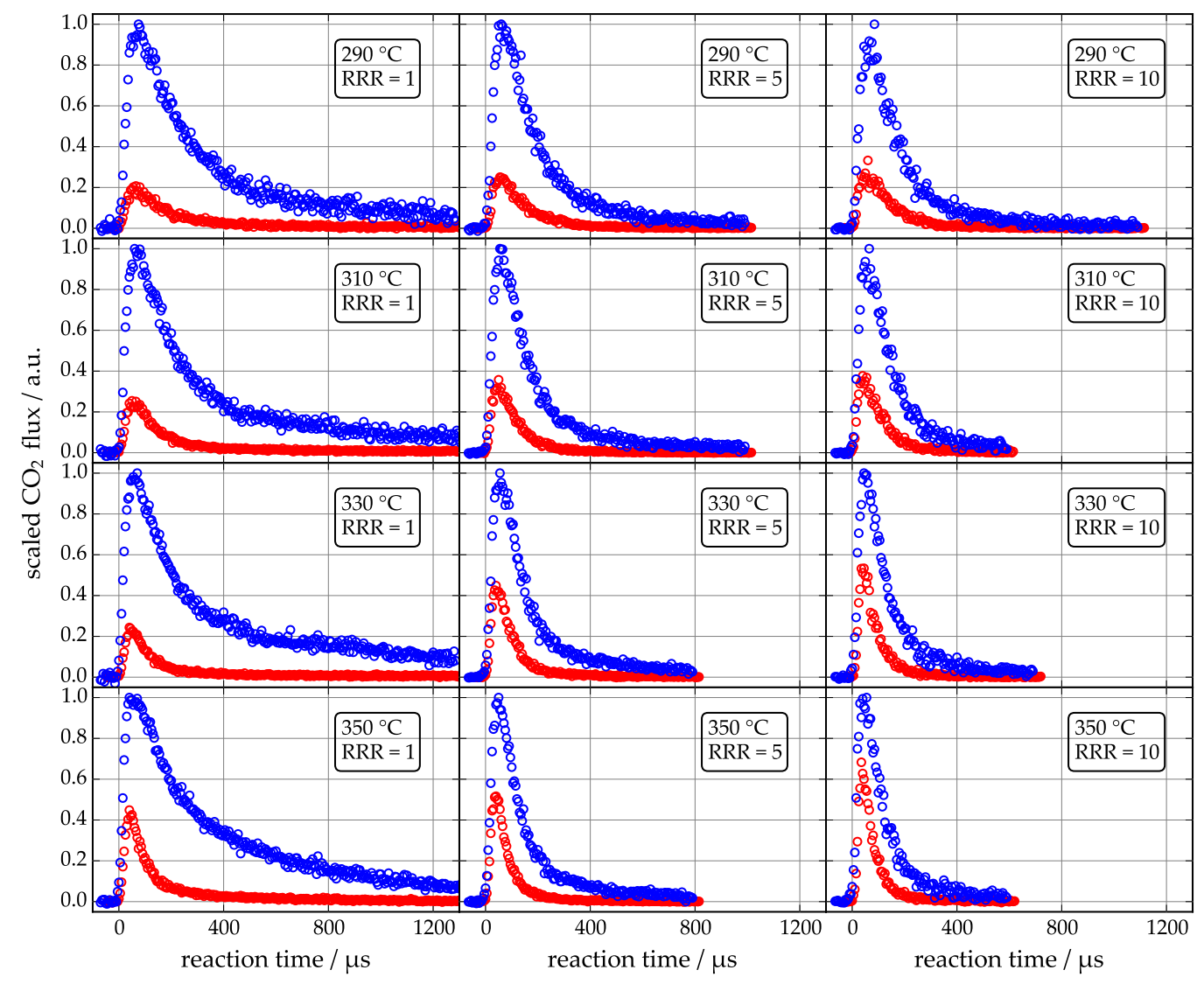

Figure 6.10: Kinetic traces at three different RRRs (horizontal) and four temperatures (vertical) are shown. The underlying scans are the same as used in the flux sum ion image overview shown in figure 6.2 (page 77) and are arranged in the same order. The time-scale of the subplots is held constant to facilitate comparison of the decay times. 
- The decay times decrease with increasing temperature, i.e. the processes leading to CO-loss become faster.

- The thermal $\mathrm{CO}_{2}$ channel is not mono-exponential, but seems to consist of two components. A fast decaying one, which dominates at early times, and a second, slower decay.

- The ratio of these two thermal components changes with temperature. Compare the traces at an RRR of 1 .

- At high temperatures and high RRRs, the thermal and hyperthermal components decay on comparable timescales.

In addition to the shape, we can compare the contribution of the thermal and the hyperthermal channel to the total flux, as shown in figure 6.11. The area of the kinetic trace of both channels was calculated for three different temperatures and at 18 RRRs for each temperature. The contribution of the thermal channel (blue markers) to the

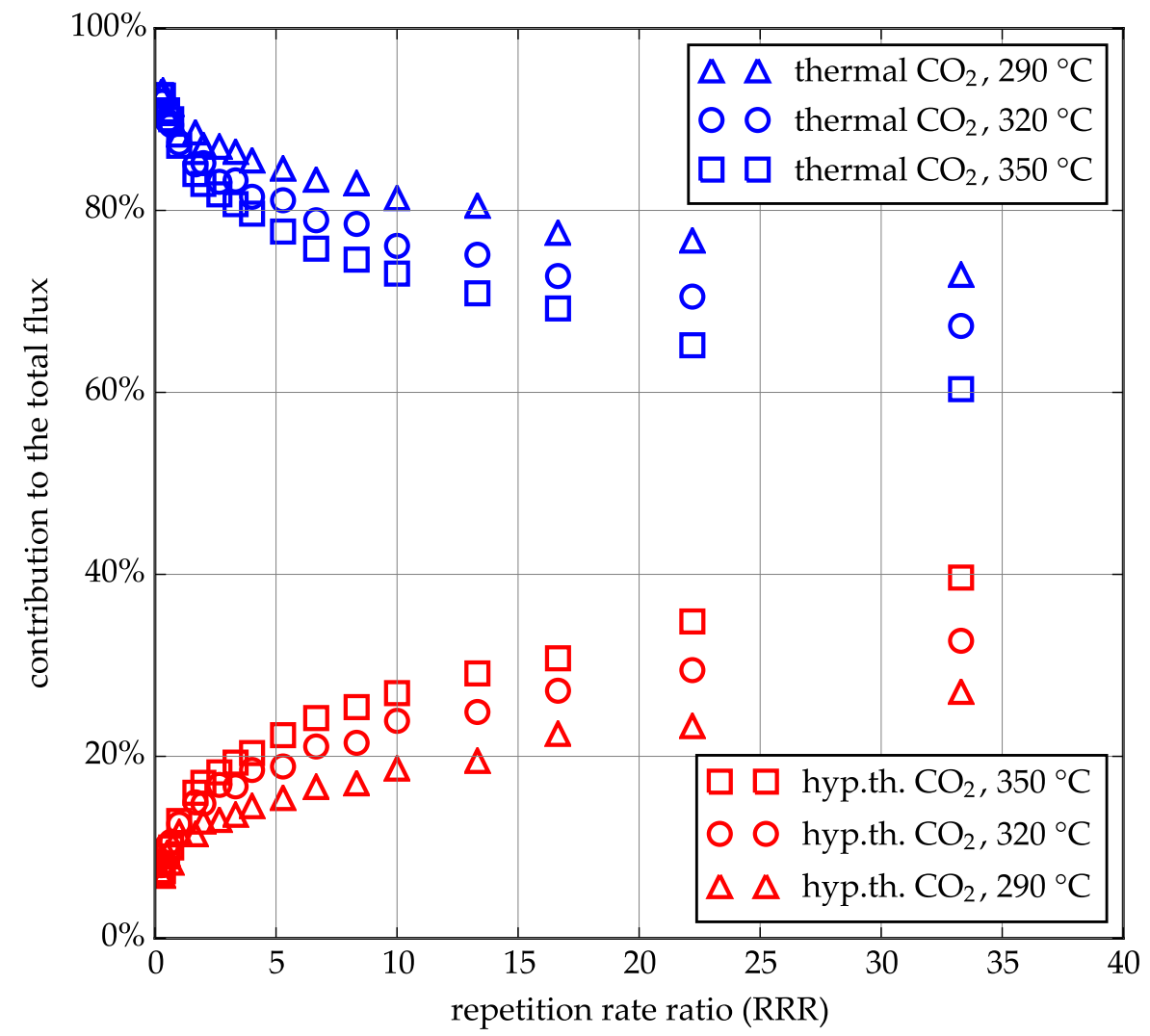

Figure 6.11: Contribution of both channels to the total flux: The relative contribution of the thermal channel is plotted as blue markers for three temperatures, the contribution of the hyperthermal (hyp.th.) channel is plotted in red.

total flux decreases with increasing RRR and with increasing temperature. Independent of temperature, the thermal channel dominates at low RRRs, contributing up to $90 \%$ of the total flux. With higher 
RRRs, the contribution decreases to about $70 \%$ at low temperatures and about $60 \%$ at high temperatures.

\subsection{KINETIC MODEL FROM EXPERIMENTAL OBSERVATIONS}

Most bimolecular reactions on surfaces can be described using either the Langmuir-Hinshelwood (LH) or the Eley-Rideal (ER) reaction model. The LH model, proposed by Langmuir (1918, [51]) and Hinshelwood (1926, [52]), describes the reaction of two chemisorbed molecules on a surface. Both reactants are in thermal equilibrium with the surface and the reaction rate depends linearly on the reactant concentrations on the surface.

The ER model describes reactions where one reactant is bound to the surface while the other reactant approaches from the gas phase. The second reactant does not adsorb on the surface and is therefore not thermally accommodated. Consequently, one revealing characteristic of an ER process is that the product shows a memory effect of the incidence angle of the second reactant. A second characteristic is that the product flux at a certain time is proportional to the flux of the gas phase reactant. For this experimental setup the product flux would have exactly the same shape as the incoming $\mathrm{CO}$ beam.

Given the long reaction time on the surface, several $\mu$ s while thermal accommodation takes place on a ns timescale, the reaction can be classified as a Langmuir-Hinshelwood (LH) type reaction, as is the consensus in literature [53-55]. The rate of a bimolecular LH reaction $\mathrm{CO}+\mathrm{O} \stackrel{\mathrm{k}_{\mathrm{r}}}{\longrightarrow} \mathrm{CO}_{2}$ can be described as

$$
\mathrm{R}=\frac{\mathrm{d}\left[\mathrm{CO}_{2}\right]}{\mathrm{dt}}=\mathrm{k}_{\mathrm{r}}[\mathrm{CO}][\mathrm{O}]
$$

Similarly, the change in CO concentration on the surface for this system can be described as

$$
\frac{\mathrm{d}[\mathrm{CO}]}{\mathrm{dt}}=-\mathrm{k}_{\mathrm{r}}[\mathrm{CO}][\mathrm{O}] \text {, }
$$

and if we add a desorption process $\mathrm{CO} \stackrel{\mathrm{k}_{\mathrm{d}}}{\longrightarrow} \mathrm{CO}_{\mathrm{g}}$ eq. 6.7 changes to

$$
\frac{d[C O]}{d t}=-k_{r}[\mathrm{CO}][\mathrm{O}]-k_{d}[\mathrm{CO}]=-(\overbrace{k_{r}[\mathrm{O}]+k_{d}}^{k_{\text {eff }}})[\mathrm{CO}] .
$$

Some of our experiments showed that the $\mathrm{CO}_{2}$ flux persists for several thousand $\mathrm{CO}$ pulses after turning off the $\mathrm{O}_{2}$ beam. From this we conclude that the $\mathrm{CO}$ flux per pulse is much smaller than the $\mathrm{O}$-atom coverage on the surface. Consequently, the change in O-atom coverage per $\mathrm{CO}$ pulse is negligible and the $\mathrm{O}$-atom coverage can be considered constant. When running both molecular beams, the (small) $\mathrm{O}$-atom consumption during the $\mathrm{CO}$ pulse is replenished by the $\mathrm{O}_{2}$ pulses. We call this a steady-state experiment - the O-atom coverage

This behaviour was seen when testing the method of initial rates. 
is constant both between $\mathrm{CO}$ pulses and during a $\mathrm{CO}$ pulse, and the $\mathrm{CO}_{2}$ flux is the same for each $\mathrm{CO}$ pulse.

Since $[\mathrm{O}]$ is time-independent, $\mathrm{k}_{\text {eff }}$ becomes a constant. We separate the variables in eq. 6.8 and integrate it as

$$
\int_{[\mathrm{CO}]_{0}}^{[\mathrm{CO}]_{t}} \frac{d[\mathrm{CO}]}{[\mathrm{CO}]}=\int_{0}^{t}-k_{\text {eff }} d t=-k_{\text {eff }} \int_{0}^{t} d t,
$$

resulting in

$$
\ln \left(\frac{[\mathrm{CO}]_{\mathrm{t}}}{[\mathrm{CO}]_{0}}\right)=-k_{\text {eff }} \mathrm{t} \quad \Longrightarrow \quad[\mathrm{CO}]_{t}=[\mathrm{CO}]_{0} e^{-k_{\text {eff }} \mathrm{t}}
$$

Substituting eq. 6.10 into eq. 6.6 yields the reaction rate as a function of time,

$$
\mathrm{R}=\frac{\mathrm{d}\left[\mathrm{CO}_{2}\right]}{\mathrm{dt}}=\mathrm{k}[\mathrm{O}][\mathrm{CO}]_{0} e^{-\mathrm{k}_{\text {eff }} \mathrm{t}}
$$

A convolution of the reaction rate over the incoming beam profile, as done in section 5.2.2 for the trapping-desorption process, could be used to fit data that decays exponentially.

This simple reaction model described above does not yet include the different $\mathrm{CO}_{2}$ channels that can be seen in the ion images. One possibility that has been discussed in literature $[49,56]$ is a bifurcation of the product $\mathrm{CO}_{2}$ after the transition state. The bifurcation ratio into thermal and hyperthermal $\mathrm{CO}_{2}$ might even change based on the chemical environment, i.e. O-atom coverage and temperature.

The kinetic traces, however, reject such a model. The two channels, as shown in figure 6.9 and 6.10, clearly have different profiles while they would share the same profile in the bifurcation model. This difference cannot be explained by an additional desorption time, which Segner et al. [49] estimated to be in the ps-range assuming a desorption prefactor on the order of $10^{13} \mathrm{~s}^{-1}$ combined with the known binding energy of $0.22 \mathrm{eV}$ for $\mathrm{CO}_{2}$ on $\mathrm{Pt}(111)$.

A second possibility, which allows the reactants to take two different reaction pathways leading to different $\mathrm{CO}_{2}$ products, can be discarded based on the same argument: The product flux would be proportional to the concentration of $\mathrm{CO}$ on the surface and the two channels would share the same lifetime.

The most simple way to explain two different lifetimes is to assume two different $\mathrm{CO}$ populations. Both the hyperthermal and the thermal channel could then be fitted with a single exponential decay function, such as eq. 6.11, convoluted over the incoming beam. An example fit to the kinetic trace from figure 6.9 is shown in figure 6.12.

The data in the right panel was plotted slightly transparent to increase visibility of the fit functions. The fit to the thermal $\mathrm{CO}_{2}$ deviates strongly, the data is clearly not single-exponential. For the hyperthermal channel the fit deviates slightly at later times. 


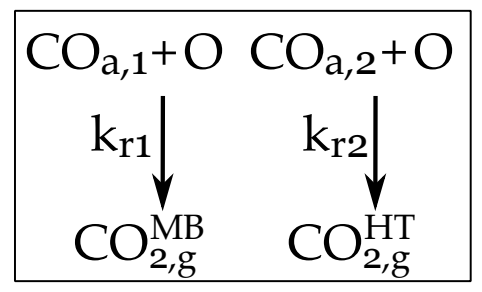

(a) Reaction scheme with two $\mathrm{CO}$ species and two reactions

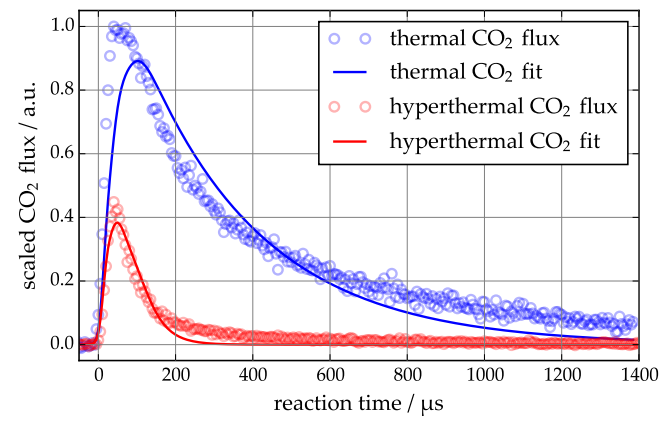

(b) Fit to the kinetic trace

Figure 6.12: (a) Reaction scheme leading to different exponential decay behaviour for the two channels. "thermal" was abbreviated th, "hyperthermal" was abbreviated hth. (b) Fit of two singleexponentials, convoluted over the incoming beam, to the kinetic trace.

In a simple trial-and-error process it was found that the thermal $\mathrm{CO}_{2}$ channel can be fitted with a bi-exponential function, where the quickly decaying component has the same lifetime as the hyperthermal channel. From this empirical result the following mechanism was suggested:

- Two different CO populations on the surface

- One population $\left(\mathrm{CO}_{\mathrm{a}, 1}\right)$ reacts with O-atoms to form thermal $\mathrm{CO}_{2}$, the other one $\left(\mathrm{CO}_{\mathrm{a}, 2}\right)$ reacts with O-atoms to form hyperthermal $\mathrm{CO}_{2}$

- $\mathrm{CO}_{\mathrm{a}, 2}$ can undergo another reaction producing thermal $\mathrm{CO}_{2}$

The reaction scheme is also depicted below in figure 6.13.

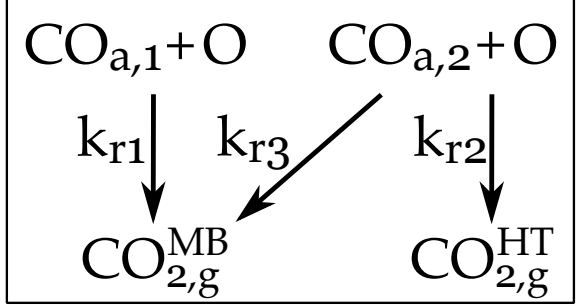

Figure 6.13: Empirical reaction scheme: Since the thermal $\mathrm{CO}_{2}$ channel has a fast-decaying component, which matches the lifetime of the hyperthermal channel, a third reaction is introduced.

While this reaction scheme is suitable to fit the data, the physical picture behind the different $\mathrm{CO}$ species and the different reactions is still unclear. One important clue to unravel this puzzle was obtained by comparing the $\mathrm{CO}$ oxidation on $\mathrm{Pt}(111)$ and on $\mathrm{Pt}(332)$. For similar experimental conditions two channels were visible on $\operatorname{Pt}(111)$ while the hyperthermal channel was absent on $\operatorname{Pt}(332)$. The salient contrast between the two surfaces is the largely different step-density - $\mathrm{Pt}(332)$ has an average terrace length of 6, the $\mathrm{Pt}(111)$ crystal used in this work had an average terrace length of about 400 Pt-atoms due to surface irregularities. We thus deduce that the thermal channel is

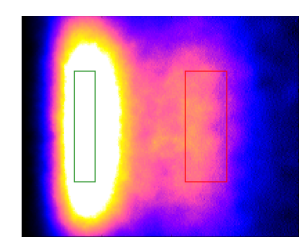

Flux image on Pt(111)

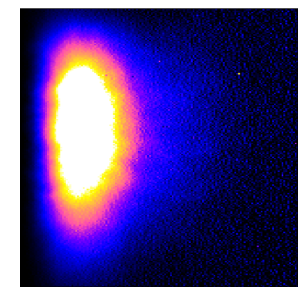

Flux image on Pt(332) 
related to step sites, and that the hyperthermal channel is related to terrace sites.

It is well known that $\mathrm{CO}$ binds about $0.16-0.35 \mathrm{eV}$ more strongly to step sites $[57,58]$ on Pt surfaces. The different binding states have even been verified in trapping-desorption experiments [41]. The more strongly bound $\mathrm{CO}$ on steps showed longer lifetimes than $\mathrm{CO}$ on terraces. It is reasonable to assume two different $\mathrm{CO}$ populations under the premise that the interchange between them is slow compared to the timescale of the experiment. The two species will henceforth be labeled $\mathrm{CO}_{\mathrm{t}}$ for $\mathrm{CO}$ bound to a terrace site and $\mathrm{CO}_{s}$ for $\mathrm{CO}$ bound to a step site. Following the lifetimes of $\mathrm{CO}$ in the trapping-desorption experiment and the relatively fast conversion of $\mathrm{CO}_{t}$ to $\mathrm{CO}_{s}$, one can predict that the long-lived reactive species producing exclusively thermal $\mathrm{CO}_{2}$ is $\mathrm{CO}$ on steps.

Similarly, different binding energies have been reported for $\mathrm{O}$-atoms at terrace and step sites [59, 6o]. We therefore distinguish between oxygen on terrace sites $\left(\mathrm{O}_{t}\right)$ and oxygen on step sites $\left(\mathrm{O}_{s}\right)$. The binding energy difference is about $0.26 \mathrm{eV}$ in favor of $\mathrm{O}_{s}$. Using the definition of the Gibbs energy,

$$
\Delta \mathrm{G}=\Delta \mathrm{H}-\mathrm{T} \Delta \mathrm{S}
$$

one can estimate the distribution of $\mathrm{O}$-atoms between terraces and steps at thermal equilibrium. On $\mathrm{Pt}(332)$ the Gibbs energy is dominated by the enthalpy $(\Delta \mathrm{H})$ term. The entropy gain for $\mathrm{O}$-atoms on terraces is only marginally higher than on steps because the density of terrace sites is greater than the density of step sites by less than an order of magnitude. On $\mathrm{Pt}(111)$ the higher ratio of terrace to step sites leads to an increased number of possible permutations on terraces, which increases the entropy gain on terrace sites.

At low oxygen coverages, as is the case for the two flux images shown above on p. 89, the O-atoms on $\mathrm{Pt}$ (332) can be expected to be almost exclusively bound to steps. With increasing O-atom coverages, and especially once the step coverage approaches saturation, the ratio of $\mathrm{O}_{t}: \mathrm{O}_{s}$ will increase. From the branching ratio on $\mathrm{Pt}(111)$ in figure 6.11 we learned that the fraction of hyperthermal $\mathrm{CO}_{2}$ increases with higher RRRs. The same trend can be seen on $\mathrm{Pt}(332)$. We now relate this behaviour to the varying ratio of $\mathrm{O}_{\mathrm{t}}: \mathrm{O}_{s}$ and conclude that the thermal $\mathrm{CO}_{2}$ is formed by reactions involving $\mathrm{O}_{\mathrm{s}}$ and the hyperthermal $\mathrm{CO}_{2}$ is formed by reactions involving $\mathrm{O}_{\mathrm{t}}$. The resulting reaction scheme is shown in figure 6.14.

This reaction scheme allows us to construct a detailed kinetic model. All relevant equations are described in the results section. 


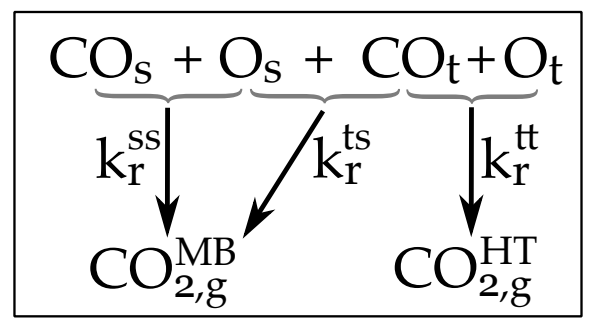

Figure 6.14: Assigned reaction scheme: The various species from the empirical reaction scheme have been assigned based on qualitative observations. $k_{r}^{s s}, k_{r}^{t s}$ and $k_{r}^{t t}$ denote reactions of $\mathrm{CO}_{s}+\mathrm{O}_{s}$, $\mathrm{CO}_{\mathrm{t}}+\mathrm{O}_{\mathrm{s}}$ and $\mathrm{CO}_{\mathrm{t}}+\mathrm{O}_{\mathrm{t}}$, respectively.

REFERENCES

[40] Kenneth C. Janda, Jerry E. Hurst, Charles A. Becker, James P. Cowin, Daniel J. Auerbach, and Lennard Wharton. "Direct measurement of velocity distributions in argon beam-tungsten surface scattering." In: The Journal of Chemical Physics 72.4 (1980), p. 2403. DOI: 10.1063/1.439434 (cit. on pp. 52, 80).

[41] Kai Golibrzuch, Pranav R. Shirhatti, Jan Geweke, Jörn Werdecker, Alexander Kandratsenka, Daniel J. Auerbach, Alec M. Wodtke, and Christof Bartels. "CO Desorption from a Catalytic Surface: Elucidation of the Role of Steps by Velocity-Selected Residence Time Measurements." In: Journal of the American Chemical Society 137.4 (2015), pp. 1465-1475. DOI: 10.1021 / ja509530k (cit. on pp. 61, 62, 70, 71, 90).

[46] Jannis Neugebohren, Dmitriy Borodin, Hinrich W. Hahn, Jan Altschäffel, Alexander Kandratsenka, Daniel J. Auerbach, Charles T. Campbell, Dirk Schwarzer, Dan J. Harding, Alec M. Wodtke, and Theofanis N. Kitsopoulos. "Velocity-resolved kinetics of site-specific carbon monoxide oxidation on platinum surfaces." In: Nature 558.7709 (June 2018), pp. 280-283. DOI: 10.1038 / s41586-018-0188-x (cit. on pp. 79-82, 123, 130, 132, 134, 146, 147).

[47] E. Poehlmann, M. Schmitt, H. Hoinkes, and H. Wilsch. "Velocity distributions of carbondioxide molecules from the oxidation of CO on Pt(111)." In: Surface Science 287-288 (May 1993), pp. 269-272. DOI: 10 . 1016 / 0039 - 6028 ( 93 ) 90784 - H (cit. on pp. 80, 81, 129, 131).

[48] K.-H. Allers, H. Pfnür, P. Feulner, and D. Menzel. "Fast Reaction Products from the Oxidation of $\mathrm{CO}$ on $\mathrm{Pt}(111)$ : Angular and Velocity Distributions of the $\mathrm{CO}_{2}$ Product Molecules." In: The Journal of Chemical Physics 100.111 (1994), pp. 3985-3998. DOI: 10.1063/1. 466332 (cit. on pp. 80, 81, 129, 131, 133, 136). 
[49] J. Segner, C. T. Campbell, G. Doyen, and G. Ertl. "Catalytic oxidation of $\mathrm{CO}$ on $\mathrm{Pt}(111)$ : The influence of surface defects and composition on the reaction dynamics." In: Surface Science 138.2-3 (Mar. 1984), pp. 505-523. DOI: 10.1016/0039-6028 (84) 90262-0 (cit. on pp. 81, 88, 96, 129, 135-137).

[50] E. Poehlmann, M. Schmitt, H. Hoinkes, and H. Wilsch. "Bimodal angular and velocity distributions of $\mathrm{CO}_{2}$ desorbing after oxidation of CO on Pt (111)." In: Surface Review and Letters 02.06 (Dec. 1995), pp. 741-758. DOI: 10.1142/S0218625X95000674 (cit. on pp. 81, 129, 133).

[51] Irving Langmuir. "The adsorption of gases on plane surfaces of glass, mica and platinum." In: Journal of the American Chemical Society 40.9 (1918), pp. 1361-1403. DOI: 10.1021/ja02242a004 (cit. on p. 87).

[52] Cyril Norman Hinshelwood. Kinetics of chemical change in gaseous systems. London: Clarendon Press, 1926 (cit. on p. 87).

[53] Charles A. Becker, James P. Cowin, Lennard Wharton, and Daniel J. Auerbach. " $\mathrm{CO}_{2}$ product velocity distributions for CO oxidation on platinum." In: The Journal of Chemical Physics 67.7 (1977), p. 3394. Dor: 10. 1063/1. 435289 (cit. on pp. 87, 131, 135).

[54] Robert L. Palmer and Joe N. Jr Smith. "Molecular beam study of CO oxidation on a (111) platinum surface." In: The Journal of Chemical Physics 60.4 (1974), p. 1453. Dor: 10.1063/1.1681219 (cit. on pp. 87, 129, 135).

[55] C. T. Campbell, G. Ertl, H. Kuipers, and J. Segner. "A Molecular Beam Study of the Catalytic Oxidation of CO on a Pt(111) Surface." In: The Journal of Chemical Physics 73.111 (1980), pp. 58625873. DOI: 10.1063/1. 440029 (cit. on pp. 87, 135, 136).

[56] Stefania Carmen Bobaru. "High-Pressure STM Studies of Oxidation Catalysis." Ph.D. Thesis. Leiden University, 2006, pp. 1118 (cit. on p. 88).

[57] J. E. Reutt-Robey, D. J. Doren, Y. J. Chabal, and S. B. Christman. "Microscopic CO Diffusion on a Pt(111) Surface by TimeResolved Infrared Spectroscopy." In: Physical Review Letters 61.24 (May 1988), pp. 2778-2781. DoI: 10 . 1103 / PhysRevLett . 61. 2778 (cit. on pp. 90, 139, 142, 143).

[58] T. H. Lin and G. A. Somorjai. "Modulated molecular beam scattering of $\mathrm{CO}$ and $\mathrm{NO}$ from $\mathrm{Pt}(111)$ and the stepped $\mathrm{Pt}$ (557) crystal surfaces." In: Surface Science 107.2-3 (1981), pp. 573-585. DOI: 10. 1016/0039-6028 (81) 90546 - X (cit. on pp. 90, 137, 142, 143). 
[59] Ryosuke Jinnouchi, Kensaku Kodama, and Yu Morimoto. “DFT calculations on $\mathrm{H}, \mathrm{OH}$ and $\mathrm{O}$ adsorbate formations on $\mathrm{Pt}(111)$ and $\mathrm{Pt}(332)$ electrodes." In: Journal of Electroanalytical Chemistry 716 (Mar. 2014), pp. 31-44. DOI: 10.1016/j . jelechem . 2013. 09. 031 (cit. on pp. 90, 112, 125, 143).

[6o] A. Nagoya, Ryosuke Jinnouchi, Kensaku Kodama, and Yu Morimoto. "DFT calculations on $\mathrm{H}, \mathrm{OH}$ and $\mathrm{O}$ adsorbate formations on $\mathrm{Pt}$ (322) electrode." In: Journal of Electroanalytical Chemistry 757 (Nov. 2015), pp. 116-127. DOI: 10.1016/j . jelechem. 2015.09.009 (cit. on pp. 90, 112, 125, 137, 143). 



\subsection{KINETIC MODEL}

\subsubsection{Reaction scheme}

The adsorption from beams is defined by the shape and intensity of the incoming beam, $\mathrm{CO}_{\text {beam }}$, and the sticking probabilities $s_{\mathrm{CO}}^{t}$ and $s_{\mathrm{CO}}^{\mathrm{s}}$ on terrace and step sites as

$$
\begin{array}{ll}
\mathrm{CO}_{\text {beam }}+\mathrm{S}_{\mathrm{t}} \stackrel{\mathrm{s}_{\mathrm{CO}}^{\mathrm{t}}}{\longrightarrow} \mathrm{CO}_{\mathrm{t}}, \\
\mathrm{CO}_{\text {beam }}+\mathrm{S}_{\mathrm{s}} \stackrel{s_{\mathrm{CO}}^{\mathrm{s}}}{\longrightarrow} \mathrm{CO}_{\mathrm{s}},
\end{array}
$$

where $S_{t}$ and $S_{s}$ denote free surface sites. Similarly the adsorption of $\mathrm{O}_{2}$ is defined as

$$
\begin{array}{lll}
\mathrm{O}_{2, \text { beam }}+2 \mathrm{~S}_{\mathrm{t}} \stackrel{\mathrm{s}_{\mathrm{O}_{2}}^{\mathrm{t}}}{\longrightarrow} 2 \mathrm{O}_{\mathrm{t}} \\
\mathrm{O}_{2, \text { beam }}+2 \mathrm{~S}_{\mathrm{s}} \stackrel{\mathrm{s}_{\mathrm{O}_{2}}^{\mathrm{s}}}{\longrightarrow} 2 \mathrm{O}_{\mathrm{s}} .
\end{array}
$$

The $\mathrm{CO}$ on terraces, $\mathrm{CO}_{\mathrm{t}}$, can desorb as

$$
\mathrm{CO}_{\mathrm{t}} \stackrel{k_{\mathrm{d}}^{\mathrm{t}}}{\longrightarrow} \mathrm{CO}_{\mathrm{g}}+\mathrm{S}_{\mathrm{t}},
$$

or it can react with a terrace oxygen atom $\mathrm{O}_{t}$ as

$$
\mathrm{CO}_{\mathrm{t}}+\mathrm{O}_{\mathrm{t}} \stackrel{\mathrm{k}_{\mathrm{r}}^{\mathrm{tt}}}{\longrightarrow} \quad \mathrm{CO}_{2, \mathrm{~g}}^{\mathrm{HT}}+2 \mathrm{~S}_{\mathrm{t}}
$$

or it can diffuse to a step, where it can encounter an empty step site and adsorb on the step,

$$
\mathrm{CO}_{\mathrm{t}}+\mathrm{S}_{\mathrm{s}} \stackrel{\mathrm{kdiff}_{\mathrm{ts}}^{\mathrm{s}}}{\longrightarrow} \mathrm{CO}_{\mathrm{s}}+\mathrm{S}_{\mathrm{t}}
$$

or encounter a step-oxygen atom $\mathrm{O}_{\mathrm{s}}$ and react,

$$
\mathrm{CO}_{\mathrm{t}}+\mathrm{O}_{\mathrm{s}} \stackrel{\mathrm{k}_{\mathrm{rs}}^{\mathrm{s}}}{\longrightarrow} \quad \mathrm{CO}_{2, \mathrm{~g}}^{\mathrm{MB}}+\mathrm{S}_{\mathrm{s}}+\mathrm{S}_{\mathrm{t}} .
$$

$\mathrm{CO}$ bound to a step site, $\mathrm{CO}_{s}$, can desorb,

$$
\mathrm{CO}_{\mathrm{s}} \quad \stackrel{k_{\mathrm{d}}^{s}}{\longrightarrow} \mathrm{CO}_{\mathrm{g}}+\mathrm{S}_{\mathrm{s}},
$$

or react with an oxygen atom at a step, $\mathrm{O}_{s}$,

$$
\mathrm{CO}_{\mathrm{s}}+\mathrm{O}_{\mathrm{s}} \stackrel{\mathrm{k}_{\mathrm{r}}^{\mathrm{s}}}{\longrightarrow} \mathrm{CO}_{2, \mathrm{~g}}^{\mathrm{MB}}+2 \mathrm{~S}_{\mathrm{s}}
$$


or diffuse back onto a terrace site,

$$
\mathrm{CO}_{s}+\mathrm{S}_{\mathrm{t}} \stackrel{\mathrm{k}_{\text {diff }}^{\text {st }}}{\longrightarrow} \mathrm{CO}_{\mathrm{t}}+\mathrm{S}_{\mathrm{s}}
$$

Note that the sticking of $\mathrm{CO}$ and $\mathrm{O}_{2}$ on $\mathrm{Pt}(111)$ is expected to happen almost exclusively on terrace sites due to the low step density of $0.25 \%$, as estimated in section 2.2. On $\mathrm{Pt}(332)$ a significant amount is expected to stick directly to step sites.

The high velocities of the hyperthermal $\mathrm{CO}_{2}$ suggest that the product molecule leaves the surface directly after passing through the transition state. Consequently, the product in equation 7.6 is a gas phase product. For the reactions 7.8 and 7.10 forming thermal $\mathrm{CO}_{2}$ an adsorbed product step between transition state and gas phase product is possible. For a binding energy of $\mathrm{CO}_{2}$ on the order of $0.22 \mathrm{eV}$, Segner et al. [49] estimated the lifetime on the surface at $650 \mathrm{~K}$ to be on the picosecond scale for an assumed prefactor of $10^{13} \mathrm{~s}^{-1}$. This is far beyond the temporal resolution of the experiment and the step involving an adsorbed product is therefore omitted.

\subsubsection{Differential equations}

This leads to a set of inhomogeneous differential equations describing the concentrations on the surface $-\mathrm{CO}_{\mathrm{t}}, \mathrm{CO}_{s}, \mathrm{O}_{\mathrm{t}}$ and $\mathrm{O}_{\mathrm{s}}-$ and the product flux. The rate equation for the change in $\mathrm{CO}_{t}$ with time is defined as

$$
\begin{aligned}
\frac{d\left[\mathrm{CO}_{t}\right]}{d t}= & -k_{d}^{t}\left[\mathrm{CO}_{t}\right]-k_{r}^{\mathrm{tt}}\left[\mathrm{CO}_{t}\right] \frac{\left[\mathrm{O}_{t}\right]}{\theta_{t}}-k_{r}^{\mathrm{ts}}\left[\mathrm{CO}_{\mathrm{t}}\right] \frac{\left[\mathrm{O}_{s}\right]}{\theta_{s}} \\
& -k_{d i f f}^{\mathrm{ts}}\left[\mathrm{CO}_{t}\right] \frac{\left[\mathrm{S}_{s}\right]}{\theta_{s}}+k_{d i f f}^{s t}\left[\mathrm{CO}_{s}\right] \frac{\left[\mathrm{S}_{t}\right]}{\theta_{t}} .
\end{aligned}
$$

Similarly, the derivative of $\mathrm{CO}_{s}$ is defined as

$$
\begin{aligned}
\frac{d\left[\mathrm{CO}_{s}\right]}{\mathrm{dt}}= & -k_{d}^{s}\left[\mathrm{CO}_{s}\right]-k_{\mathrm{r}}^{\mathrm{ss}}\left[\mathrm{CO}_{s}\right] \frac{\left[\mathrm{O}_{s}\right]}{\theta_{s}} \\
& +k_{d i f f}^{\mathrm{ts}}\left[\mathrm{CO}_{\mathrm{t}}\right] \frac{\left[\mathrm{S}_{\mathrm{t}}\right]}{\theta_{s}}-k_{\text {diff }}^{s t}\left[\mathrm{CO}_{s}\right] \frac{\left[\mathrm{S}_{\mathrm{t}}\right]}{\theta_{\mathrm{t}}} .
\end{aligned}
$$

In both equations the square brackets denote reactant concentrations in ML with respect to the entire surface. The total terrace and step (empty and filled) concentrations $\theta_{\mathrm{t}}$ and $\theta_{\mathrm{s}}$ are also expressed in ML with respect to the entire surface. The fractions in the equations above therefore become unitless and all rate constants $k$ are expressed in units of $\mathrm{s}^{-1}$.

The derivative of the concentration of oxygen on terraces,

$$
\frac{\mathrm{d}\left[\mathrm{O}_{\mathrm{t}}\right]}{\mathrm{dt}}=-\mathrm{k}_{\mathrm{r}}^{\mathrm{tt}}\left[\mathrm{CO}_{\mathrm{t}}\right] \frac{\left[\mathrm{O}_{\mathrm{t}}\right]}{\theta_{\mathrm{t}}}
$$

and the derivative of the concentration of oxygen on steps, 


$$
\frac{\mathrm{d}\left[\mathrm{O}_{\mathrm{s}}\right]}{\mathrm{dt}}=-\mathrm{k}_{\mathrm{r}}^{\mathrm{ts}}\left[\mathrm{CO}_{\mathrm{t}}\right] \frac{\left[\mathrm{O}_{\mathrm{s}}\right]}{\theta_{\mathrm{s}}}-\mathrm{k}_{\mathrm{r}}^{\mathrm{ss}}\left[\mathrm{CO}_{\mathrm{s}}\right] \frac{\left[\mathrm{O}_{\mathrm{s}}\right]}{\theta_{\mathrm{s}}}
$$

both neglect oxygen desorption. For the temperature range studied, the recombinative desorption rate for $\mathrm{O}$ on $\mathrm{Pt}(111)$ is at least a factor of $10^{4}$ smaller than the desorption of $\mathrm{CO}$ from steps, which is the slowest process in the model. The diffusion of oxygen between terrace and step sites while the reaction takes place is neglected for the same reason: For a diffusion barrier of $1.2 \mathrm{eV}$ and a diffusion coefficient of $2 \times 10^{-4} \mathrm{~cm} \mathrm{~s}^{-2}$ [62] we can estimate the time it takes to travel the distance of a single $\mathrm{Pt}$ atom to be $250 \mu \mathrm{s}$.

Additionally, the change in free terrace and step sites, $S_{t}$ and $S_{s}$, is defined as

$$
\begin{aligned}
\frac{d\left[S_{t}\right]}{d t}= & +k_{d}^{t}\left[\mathrm{CO}_{t}\right]+2 k_{r}^{t t}\left[\mathrm{CO}_{t}\right] \frac{\left[\mathrm{O}_{t}\right]}{\theta_{t}}+k_{r}^{\mathrm{ts}}\left[\mathrm{CO}_{\mathrm{t}}\right] \frac{\left[\mathrm{O}_{s}\right]}{\theta_{s}} \\
& +k_{d i f f}^{\mathrm{ts}}\left[\mathrm{CO}_{t}\right] \frac{\left[\mathrm{S}_{s}\right]}{\theta_{s}}-k_{d i f f}^{\mathrm{st}}\left[\mathrm{CO}_{s}\right] \frac{\left[\mathrm{S}_{\mathrm{t}}\right]}{\theta_{t}}
\end{aligned}
$$

and

$$
\begin{aligned}
\frac{d\left[S_{s}\right]}{d t}= & +k_{d}^{s}\left[\mathrm{CO}_{s}\right]+2 k_{r}^{s s}\left[\mathrm{CO}_{s}\right] \frac{\left[\mathrm{O}_{s}\right]}{\theta_{t}}+k_{r}^{\mathrm{ts}}\left[\mathrm{CO}_{t}\right] \frac{\left[\mathrm{O}_{s}\right]}{\theta_{s}} \\
& -k_{d i f f}^{\mathrm{ts}}\left[\mathrm{CO}_{t}\right] \frac{\left[\mathrm{S}_{s}\right]}{\theta_{s}}+k_{d i f f}^{s t}\left[\mathrm{CO}_{s}\right] \frac{\left[\mathrm{S}_{t}\right]}{\theta_{t}}
\end{aligned}
$$

Lastly, we can formulate the flux of thermal (MB) $\mathrm{CO}_{2}$,

$$
\operatorname{Flux}\left(\mathrm{CO}_{2, \mathrm{~g}}^{\mathrm{MB}}\right)=k_{\mathrm{r}}^{\mathrm{ts}}\left[\mathrm{CO}_{\mathrm{t}}\right] \frac{\left[\mathrm{O}_{\mathrm{s}}\right]}{\theta_{\mathrm{s}}}+\mathrm{k}_{\mathrm{r}}^{\mathrm{ss}}\left[\mathrm{CO}_{\mathrm{s}}\right] \frac{\left[\mathrm{O}_{\mathrm{s}}\right]}{\theta_{\mathrm{s}}}
$$

and the flux of hyperthermal (HT) $\mathrm{CO}_{2}$,

$$
\operatorname{Flux}\left(\mathrm{CO}_{2, \mathrm{~g}}^{\mathrm{HT}}\right)=\mathrm{k}_{\mathrm{r}}^{\mathrm{tt}}\left[\mathrm{CO}_{\mathrm{t}}\right] \frac{\left[\mathrm{O}_{\mathrm{t}}\right]}{\theta_{\mathrm{t}}} \text {. }
$$

These differential equations constitute a nonlinear initial value problem which cannot be solved analytically. For example, the change in $\left[\mathrm{CO}_{t}\right]$ contains $\left[\mathrm{CO}_{s}\right]$ and vice versa. An analytic solution can only be provided under drastic assumptions - both constant $\left[\mathrm{O}_{\mathrm{t}}\right]$ and $\left[\mathrm{O}_{s}\right]$, constant number of free sites $\left[\mathrm{S}_{\mathrm{t}}\right]$ and $\left[\mathrm{S}_{\mathrm{t}}\right]$, and excluding diffusion of $\mathrm{CO}$ from steps back to terraces. Since the validity of these assumptions is questionable, we instead chose to solve the system numerically.

\subsubsection{Numeric approximation to differential equations}

The simplest numerical procedure to solve a system of ordinary differential equations (ODEs) has been described by Euler in 1768. This method uses a starting point $\mathrm{C}_{0}$, in our case an initial concentration, 
and calculates the slope at this point. For our system the variable is the time $t$ and the slope $\frac{d C_{0}}{d t}$ is calculated from the respective differential equation using the starting concentrations. This slope is multiplied by a small time step $\Delta \mathrm{t}$ which yields the increment $\frac{\mathrm{dC}_{\mathrm{o}}}{\mathrm{dt}} \times \Delta \mathrm{t}$ and we calculate the concentration at $t_{1}=t_{0}+\Delta t$ as

$$
\mathrm{C}_{1}=\mathrm{C}_{0}+\frac{\mathrm{dC} 0}{\mathrm{dt}} \times \Delta \mathrm{t}
$$

The differential equations are then re-evaluated using the new concentrations and by repetition one can obtain an approximate solution to the initial value problem. The local truncation error scales proportional to the square of the step size $\Delta \mathrm{t}^{2}$ and the total accumulated error scales proportionally to the step-size, which makes it a firstorder method. It is best to use very small steps which can become computationally expensive. More accurate results can be obtained by using a predictor-corrector method such as the trapezoidal method. This method uses the result of the Euler method as a prediction to obtain the concentration $\tilde{C}_{1}=C_{0}+\Delta_{1}$ at $t_{1}$, calculates the next increment $\Delta_{2}$ and then interpolates the derivative by using the average of these two increments for the first time step,

$$
\mathrm{C}_{1}=\mathrm{C}_{0}+\frac{1}{2}\left(\frac{\mathrm{d} \mathrm{C}_{0}}{\mathrm{dt}} \times \Delta \mathrm{t}+\frac{\mathrm{d} \tilde{\mathrm{C}}_{1}}{\mathrm{dt}} \times \Delta \mathrm{t}\right)
$$

where $\tilde{C}_{1}$ is the concentration as calculated by Euler's method. While the process above is a first-order interpolation, methods such as the Adam's method can be extended to higher-order interpolation yielding better accuracy. Higher precision can be achieved by using higherorder methods, such as the widely used Runge-Kutta method. The fourth-order Runge-Kutta ( $\left.\mathrm{RK}_{4}\right)$ calculates the increment in four different ways and weighs them as follows:

- increment $\Delta_{1}$ at the starting point of the time step using $C_{0}$, weighted $1 / 6$

- increment $\Delta_{2}$ at the middle of the time step using the concentration $\mathrm{C}_{0}+0.5 \Delta_{1}$, weighted $2 / 6$

- increment $\Delta_{3}$ at the middle of the time step using the concentration $C_{0}+0.5 \Delta_{2}$, weighted $2 / 6$

- increment $\Delta_{4}$ at the end of the time step using the concentration $\mathrm{C}_{0}+\Delta_{3}$, weighted $1 / 6$

The use of the weighted average of these increments, with greater weigth on the middle increments $\Delta_{2}$ and $\Delta_{3}$, greatly increases the accuracy of the method. The total accumulated error is proportional to $\Delta \mathrm{t}^{4}$, which means that the numeric approximation converges with the 4 th power of the number of steps. 
Another problem to be aware of when solving an initial value problem is the stiffness of the system. In Numerical Methods for Ordinary Differential Systems [63] J. D. Lambert defines the stiffness as follows:

If a numerical method with a finite region of absolute stability, applied to a system with any initial conditions, is forced to use in a certain interval of integration a steplength which is excessively small in relation to the smoothness of the exact solution in that interval, then the system is said to be stiff in that interval.

In other words, one would expect to need a small step size in a region where the solution exhibits large variations and large step sizes where the solution straightens out. For some systems this is not the case, these systems are considered stiff. Computationally cheap methods such as the Adam's method are good for solving non-stiff problems, but more elaborate methods and smaller time steps are necessary to solve stiff systems.

For this work I used the LSODA routine from the FORTRAN77 library ODEPACK [64]. Besides being a computationally-optimized fortran code, the method automatically selects between non-stiff (Adams) and stiff (BDF) methods, based on the behavior of the data. On top of that, the LSODA routine adapts the time step size dynamically based on the data. Per time step, e.g. every $1 \mu \mathrm{s}$, the solver is by default allowed to calculate up to 1000 solver steps. These features make the solver both fast and reliable.

\subsubsection{Perturbation of the equilibrium by incoming beams}

The system as outlined above can be used to solve an initial value problem. In our experiment, however, there is no initial $\mathrm{CO}$ concentration on the surface. Instead, the $\mathrm{CO}$ dose follows the profile of the incoming $\mathrm{CO}$ pulse and the shape of the pulse has to be taken into account. The differential equations determining the surface concentrations can be divided into two categories: The change in concentrations due to the differential equations from section 7.1.2 depends only on the concentrations on the surface. This set of differential equations can be considered homogeneous. The change in concentration introduced by the incoming CO pulse, however, is not purely a function of the present concentrations on the surface. The perturbation of concentrations on the surface can therefore be considered inhomogeneous.

\subsubsection{Adsorption from incoming beams}

The inhomogeneous part of the kinetic model, i.e. the perturbation of the system through molecular beams, is described in the following 


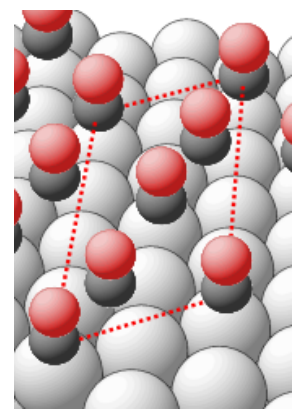

$\mathrm{c}(4 \times 2) \mathrm{CO}$ unit cell

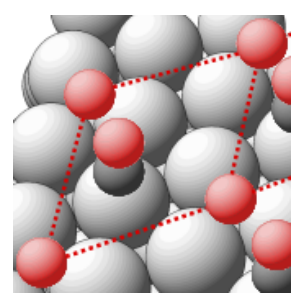

$c(2 \times 2)$ $\mathrm{O}+\mathrm{CO}$ unit cell

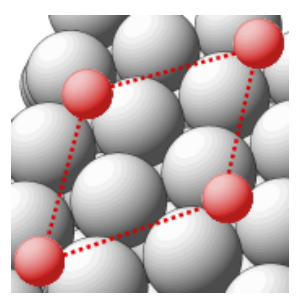

$(2 \times 2)$ O unit cell differential equations for $\operatorname{Pt}(111)$. The adsorption of $\mathrm{CO}$ on terraces is defined as

$$
\frac{\mathrm{d}\left[\mathrm{CO}_{\mathrm{t}}\right]}{\mathrm{dt}}=\mathrm{s}_{\mathrm{CO}}^{0} \times \mathrm{CO}_{\text {beam }}\left(1-\theta_{\mathrm{s}}-2\left[\mathrm{O}_{\mathrm{t}}\right]-2\left[\mathrm{CO}_{\mathrm{t}}\right]\right),
$$

with the initial sticking coefficient $s_{\mathrm{CO}}^{0}=0.86$. The bracket is equivalent to the amount of free terrace sites. According to this, the maximum concentration of $\mathrm{CO}$ on an otherwise bare surface would be $\left[\mathrm{CO}_{\mathrm{t}}\right]=0.5 \mathrm{ML}$. This agrees with the $\mathrm{c}(4 \times 2)$ unit cell as shown in the figure in the margin, which is consensus in literature $[38,65-67]$. In a $c(4 \times 2)$ unit cell two top sites and two bridge sites are populated.

Both the step concentration $\theta_{s}$ on $\operatorname{Pt}(111)$ and the adsorbed $\left[\mathrm{CO}_{t}\right]$ are small at our experimental conditions, so the last factor in equation 7.22 is dominated by $\left(1-2\left[\mathrm{O}_{t}\right]\right)$. This makes the effective sticking in the kinetic model mostly a function of the O-atom coverage.

Oxygen on $\mathrm{Pt}(111)$ dissociatively adsorbs in $(2 \times 2)$ unit cells with a maximum coverage of $\left[\mathrm{O}_{\mathrm{t}}\right]=0.25 \mathrm{ML}$ on terraces, as shown in the bottom picture. There is one $\mathrm{CO}$ adsorption site in a $(2 \times 2)$ oxygen lattice, as shown in the second picture. This is half the available sites compared to the $c(4 \times 2)$ unit cell in the top picture, as reported by Liu et al. [68]. This physical picture agrees with equation 7.22 , where the bracket evaluates to $\approx 1$ for low $\left[\mathrm{O}_{\mathrm{t}}\right]$ and to $\approx 0.5$ for high $\left[\mathrm{O}_{\mathrm{t}}\right]$. The maximum amount of $\mathrm{CO}$ coadsorbed in a $(2 \times 2)$ oxygen unit cell is $\left[\mathrm{CO}_{\mathrm{t}}\right]=0.25 \mathrm{ML}$. The adsorption of bridge bonded $\mathrm{CO}$ is supressed by $0.25 \mathrm{ML}$ of atomic oxygen [67].

The dissociative adsorption of $\mathrm{O}_{2}$ is defined in a similar way,

$$
\frac{\mathrm{d}\left[\mathrm{O}_{\mathrm{t}}\right]}{\mathrm{dt}}=2 \times \mathrm{s}_{\mathrm{O}_{2}}^{0} \times \mathrm{O}_{2, \text { beam }}\left(1-\theta_{\mathrm{s}}-4\left[\mathrm{O}_{\mathrm{t}}\right]-2\left[\mathrm{CO}_{\mathrm{t}}\right]\right)^{2},
$$

except that one $\mathrm{O}_{2}$ molecule needs two adsorption sites and therefore the sticking probability scales with the square of the free sites. The last term, dominated by $\left(1-4\left[\mathrm{O}_{\mathrm{t}}\right]\right)$, allows a maximum $\left[\mathrm{O}_{\mathrm{t}}\right]$ concentration of $\left[\mathrm{O}_{\mathrm{t}}\right]=0.25 \mathrm{ML}$ on $\mathrm{Pt}(111)$ in a $(2 \times 2)$ unit cell, as shown in the bottom picture.

\subsection{OXYGEN TITRATION EXPERIMENT}

\subsubsection{Calibration on a fully covered surface}

The total amount of oxygen on the surface, $\left[\mathrm{O}_{\mathrm{a}}\right]=\left[\mathrm{O}_{\mathrm{t}}\right]+\left[\mathrm{O}_{\mathrm{s}}\right]$, was determined in a titration [69] experiment. The procedure consists of two steps: A calibration using a fully covered surface, and the measurement on a surface with unknown oxygen concentration. The calibration on a fully covered surface was conducted as follows:

1. The surface is dosed with oxygen for several minutes. A high repetition rate of $333 \mathrm{~Hz}$ was used to ensure full oxygen cover- 
age is achieved after this time. The recombinative desorption of oxygen at higher temperatures of $700-750 \mathrm{~K}$ is on the order of minutes [7o]. At the titration temperature $\left(61_{3} \mathrm{~K}\right)$ the desorption could be neglected as long as no time was wasted.

2. The $\mathrm{O}_{2}$ beam is turned off.

3. A dark image is recorded and a scan is started using Davis software. This is done within less than 20 seconds after turning off the beam.

4. The $\mathrm{CO}$ beam is turned on and the $\mathrm{CO}_{2}$ production at a certain beam-laser delay is recorded until the signal vanishes.

5. The first 4 steps are repeated several times for different beamlaser delays. See explanation in section 7.2.2.

6. The total $\mathrm{CO}_{2}$ yield is calculated by integrating the sum of all scans. This amount of $\mathrm{CO}_{2}$ was produced by reacting $0.25 \mathrm{ML}$ of oxygen.

During the titration all possible oxygen coverages between 0.25 ML and o ML are probed. The titration starts at $\left[\mathrm{O}_{\mathrm{a}}\right]=0.25 \mathrm{ML}$ and the surface concentration decreases as $\mathrm{O}$-atoms are consumed in the reaction. We can qualitatively compare the conditions at the beginning of the titration to the highest obtainable concentration $\left[\mathrm{O}_{a}\right]$ in kinetic trace measurements. Higher RRRs lead to higher O-atom concentrations on the surface. $\left[\mathrm{O}_{a}\right]$ at the highest RRR should then be comparable to the beginning of the titration. As the titration continues, and as $\left[\mathrm{O}_{\mathrm{a}}\right]$ decreases, the reaction conditions should be comparable to the steady-state $\left[\mathrm{O}_{a}\right]$ value of increasingly smaller RRRs.

\subsubsection{From titrations to total $\mathrm{CO}_{2}$ yield — dimensionality problem}

In a kinetic trace measurement, we vary the beam-laser delay while $\left[\mathrm{O}_{\mathrm{a}}\right]$ is constant. This allows us to measure the time-resolved $\mathrm{CO}_{2}$ production following a single pulse of $\mathrm{CO}$. The thermal channel is plotted in figure 7.I for five kinetic traces at different RRRs and $593 \mathrm{~K}$.

In a titration, however, we cannot scan over multiple beam-laser delays because the oxygen concentration changes with each pulse of $\mathrm{CO}$. Instead, we measure the $\mathrm{CO}_{2}$ flux at a fixed beam-laser delay (reaction time). This process can be visualized by comparing the flux values at a reaction time of e.g. $100 \mu \mathrm{s}$. The kinetic traces in figure 7.1 have all been normalized with respect to the maximum in order to illustrate the following point. Consequently, the $\mathrm{CO}_{2}$ flux at a reaction time of $100 \mu \mathrm{s}$ is near the maximum value of 1 for all traces. The values measured at this point, however, are not proportional to the areas under the respective curve of the kinetic trace because of the

Reminder: higher $R R R$ means higher $\mathrm{O}_{2}: \mathrm{CO}$ flux ratio. 


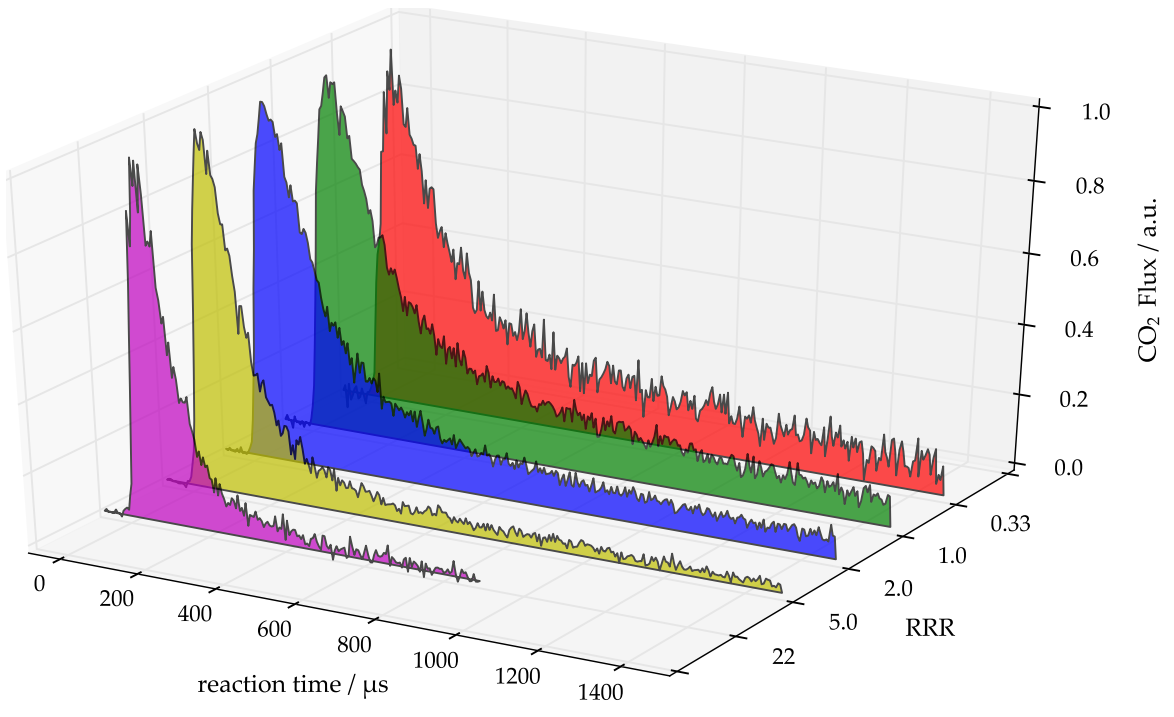

Figure 7.1: Comparison of kinetic traces of the thermal channel: Five kinetic traces at $593 \mathrm{~K}$ and for different RRRs are shown.

varying shape! Therefore the measured $\mathrm{CO}_{2}$ flux at a fixed delay at any point in the titration is not proportional to the total $\mathrm{CO}_{2}$ flux produced by that $\mathrm{CO}$ pulse.

The quantity of interest is the total $\mathrm{CO}_{2}$ production during a titration, which can be compared to the volume under the area spanned by the kinetic traces in figure 7.I after interpolating the missing RRRs. A titration at a reaction time is a $2 \mathrm{D}$-slice parallel to the RRR-axis. In order to reconstruct the $3 \mathrm{D}$ volume, several titrations at different reaction times are necessary.

This problem is further illustrated in figure 7.2. The two traces shown are the ones labeled $R R R=1$ (top) and an $R R R=22$ (bottom) from figure 7.I. The left panel is synonymous to measuring the titration at a single reaction time of $100 \mu \mathrm{s}$ and assuming that the $\mathrm{CO}_{2}$ flux at that delay is an accurate representation of the area of the kinetic trace. At $100 \mu$ s both kinetic traces are close to the max-

This example compares two normalized traces. The relative error would be the same for non-normalized traces though! imum value. The rectangle represents the resulting estimate of the area, which would then be the same for the two traces despite their different shape. The ratio of the areas as estimated from the single titration at a reaction time of $100 \mu$ s would be $1: 1$ for the two kinetic traces, while the actual ratio green to magenta should be 2.62:1. In other words, the titration would increasingly underestimate the $\mathrm{CO}_{2}$ flux as the titration goes on and shifts from high coverage conditions (magenta) to low coverage conditions (green).

The right panel shows the situation when the titration is repeated at 5 different delays. The signal from the five titrations can simply be added, which is equivalent to representing the area under the kinetic trace by a sum of rectangles of equal width. Note that this leads to a much more accurate representation of the $\mathrm{CO}_{2}$ flux, with an es- 

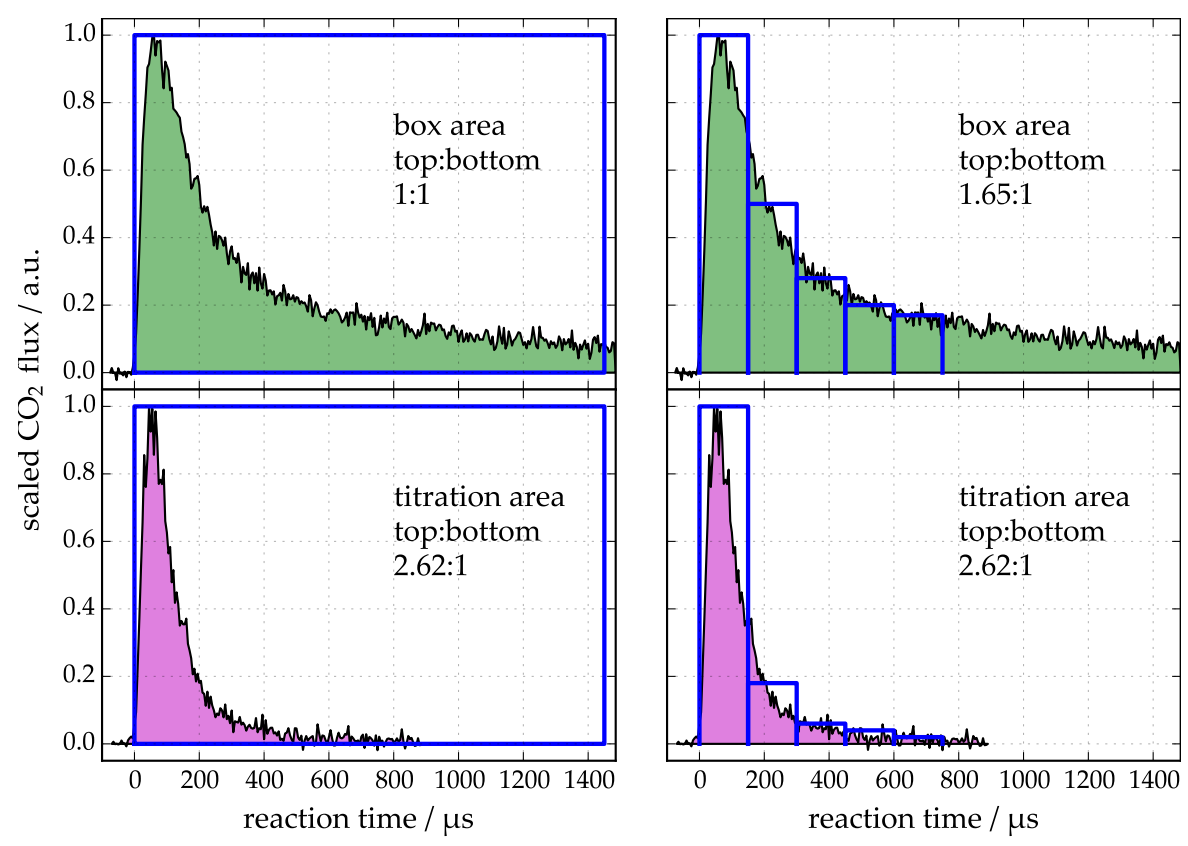

Figure 7.2: During a titration the $\mathrm{CO}_{2}$ flux following each $\mathrm{CO}$ pulse is measured at a fixed beam-laser delay. The left and right panel compare how well this $\mathrm{CO}_{2}$ flux relates to the total $\mathrm{CO}_{2}$ produced during the titration. Left: Usage of only one titration at a a single reaction time is comparable to estimation of the area under the kinetic trace from a single value. Right: Estimate using five titrations at different reaction times. Simply adding these five titrations can be compared to approximating the area under the kinetic trace by 5 rectangles of equal width. 
A high temperature with short kinetic traces was chosen when testing the technique.

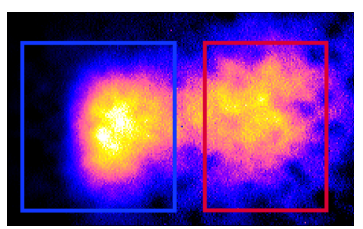

flux sum ion image from the titration timated ratio of 1.65:1 for the qualitatively chosen rectangles above. Multiple titrations at different reaction times are better suited to capture the total $\mathrm{CO}_{2}$ production throughout the titration.

\subsection{3 $\mathrm{CO}_{2}$ flux vs titration time}

An example set of titrations at $653 \mathrm{~K}$ is shown in figure 7.3. The $\mathrm{CO}_{2}$ flux as a function of titration time was recorded at five different beamlaser delays and the thermal (MB) and hyperthermal (HT) fluxes are plotted as blue and red lines. The thermal channel signal was scaled to correctly reflect the broader angular distribution. The beam-laser delay, given in the respective legend for the top five plots, is not converted into reaction time because the reaction time is not the same for all molecules on an ion image. An example flux sum ion image with integration regions is shown on the side. Choosing a velocityresolved area during the extraction, which is an essential requirement when converting to reaction time, would only reduce the signal-tonoise ratio (SNR) and not provide any benefits.

At the beam-laser delay of $160 \mu$ s in the top panel, which is only tens of $\mu$ s after the CO pulse arrived at the surface (at $t \approx 140$ to $150 \mu \mathrm{s}$ ), the hyperthermal $\mathrm{CO}_{2}$ dominates due to its shorter flight time. The signal of the hyperthermal channel decreases quickly within the first 500-1000 CO pulses of the titration. The $\mathrm{CO}$ pulsed valve was triggered with a repetition rate of $20 \mathrm{~Hz}$. A titration time of $100 \mathrm{~s}$ then corresponds to 2000 pulses of CO.

The thermal channel, which dominates at the other beam-laser delays, shows almost constant signal for the first 500 pulses of $\mathrm{CO}$ where the hyperthermal channel exhibits a significant decrease. The $\mathrm{CO}_{2}$ yield of the thermal channel then decreases slowly over the next 2000 pulses.

It took approximately $2000 \mathrm{CO}$ pulses to remove $0.25 \mathrm{ML}$ of oxygen atoms. As a secondary outcome of the titration measurement this allows us to estimate the dose per $\mathrm{CO}$ pulse as

$$
\begin{aligned}
\frac{0.25 \mathrm{ML}}{2000 \text { pulses }} & =1.2 \times 10^{-4} \mathrm{ML} / \text { pulse } \\
& \equiv 1.9 \times 10^{11} \text { molecules/pulse, }
\end{aligned}
$$

which represents a lower limit to the $\mathrm{CO}$ flux for a sticking probability of $I$ and a reaction probability of 1 .

By adding the $\mathrm{CO}_{2}$ production of the hyperthermal and the angular distribution corrected thermal channel for all five titrations, the added $\mathrm{CO}_{2}$ yield as shown in the bottom panel in figure 7.3 is obtained. Integrating over the duration of the titration yields the total

\author{
amount of $\mathrm{CO}_{2}$ produced.
}

The thermal channel flux was multiplied by a factor of 4.7 . 


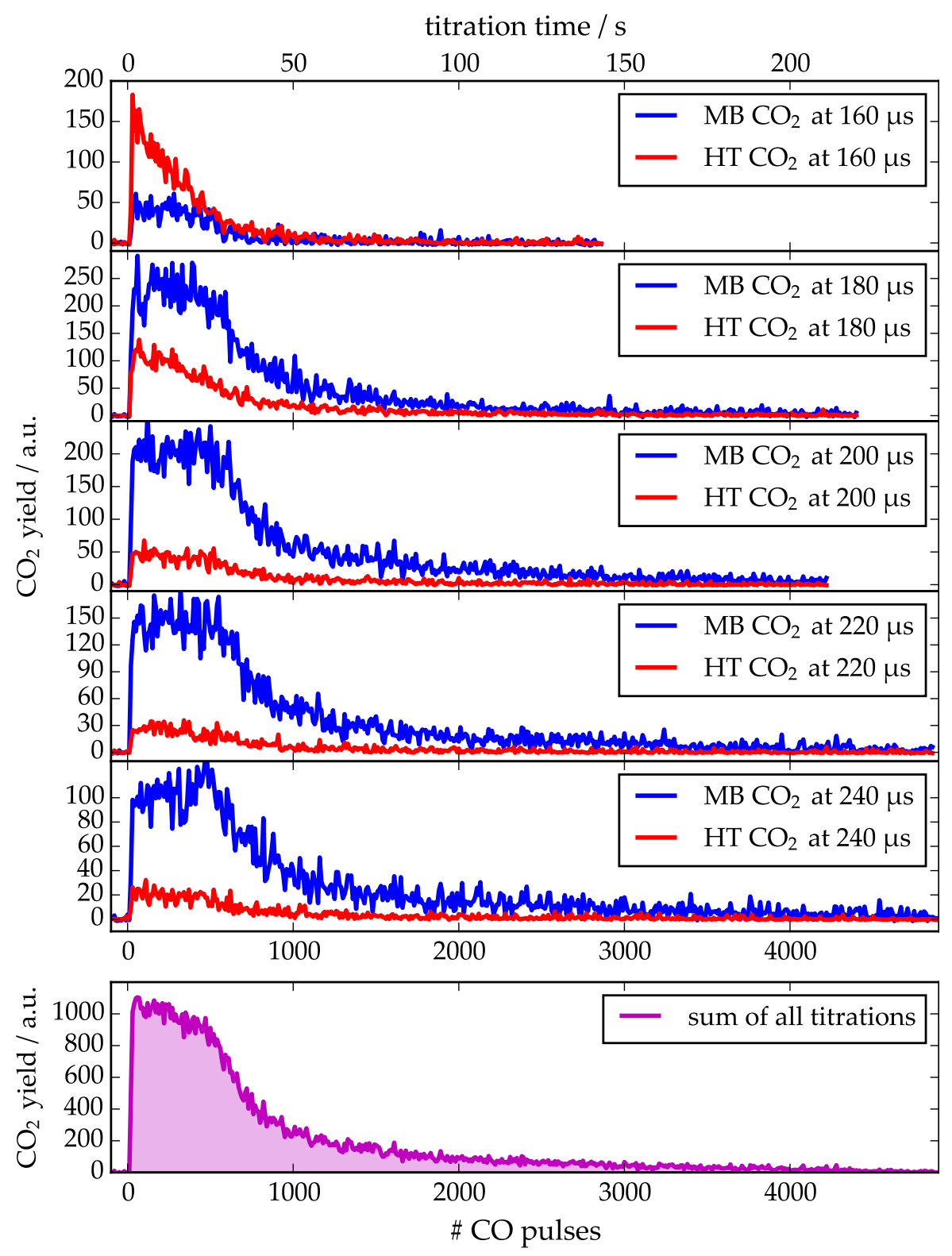

Figure 7.3: The top five panels show the thermal (MB) and hyperthermal (HT) $\mathrm{CO}_{2}$ flux for titrations measured at different beam-laser delays as given in the respective legend. The titrations shown here were recorded with a $\mathrm{CO}$ repetition rate of $20 \mathrm{~Hz}$ at $653 \mathrm{~K}$ and a starting concentration of $0.25 \mathrm{ML} \mathrm{O}$-atoms. The bottom panel shows the sum of all traces. The area under the curve, which is the quantity of interest, is highlighted in bright magenta. 


\subsubsection{Titration of a partially covered surface}

Now that the titration has been calibrated on a fully covered surface, it can be used to measure the $\mathrm{O}$-atom coverage on a partially covered surface. The determination of the steady-state $\mathrm{O}$-atom coverage per RRR was conducted as follows:

1. Both molecular beams are run at a certain RRR for several minutes to establish steady-state $\mathrm{O}$-atom coverage.

2. Both molecular beams are turned off at the same time.

3. The CO pulsed valve repetition rate is set to the same value as used in the calibration of the titration, i.e. $20 \mathrm{~Hz}$ for most titrations.

4. A dark image is recorded and a scan is started using Davis software. This is done within less than 20 seconds after turning off the beams.

5. The $\mathrm{CO}$ beam is turned on and the $\mathrm{CO}_{2}$ production at a certain beam-laser delay is recorded until the signal vanishes.

6. The first 5 steps are repeated several times for different beamlaser delays.

7. The total $\mathrm{CO}_{2}$ yield is calculated by integrating the sum of all scans.

8. By comparing this yield to the yield from a fully covered surface, the steady-state O-atom coverage is determined.

Both the calibration and the measurements were done at $613 \mathrm{~K}$ and several RRRs for $\mathrm{Pt}(111)$. On $\mathrm{Pt}(332)$ the steady-state coverage was measured for 6 different RRRs and additionally the possible temperature dependence was briefly investigated.

\subsubsection{Result: O-atom coverage vs RRR}

The resulting O-atom coverage as a function of $\mathrm{RRR}$ for $\operatorname{Pt}(111)$ is shown in figure 7.4. The uncertainty of the result due to erroneous reproduction of the total $\mathrm{CO}_{2}$ flux can be estimated by comparing how much the result for one RRR changes if only 4 out of 5 beamlaser delays in a set of titrations are taken into account. Increasing the number of different beam-laser delays should further improve the accuracy. The uncertainty introduced here is a relative amount (a percentage) of the determined coverage. On top of that the correct determination of the baseline has a major impact on the area under the curve for both calibration and steady-state measurement. The uncertainty from this factor is an absolute amount. The error bars were 


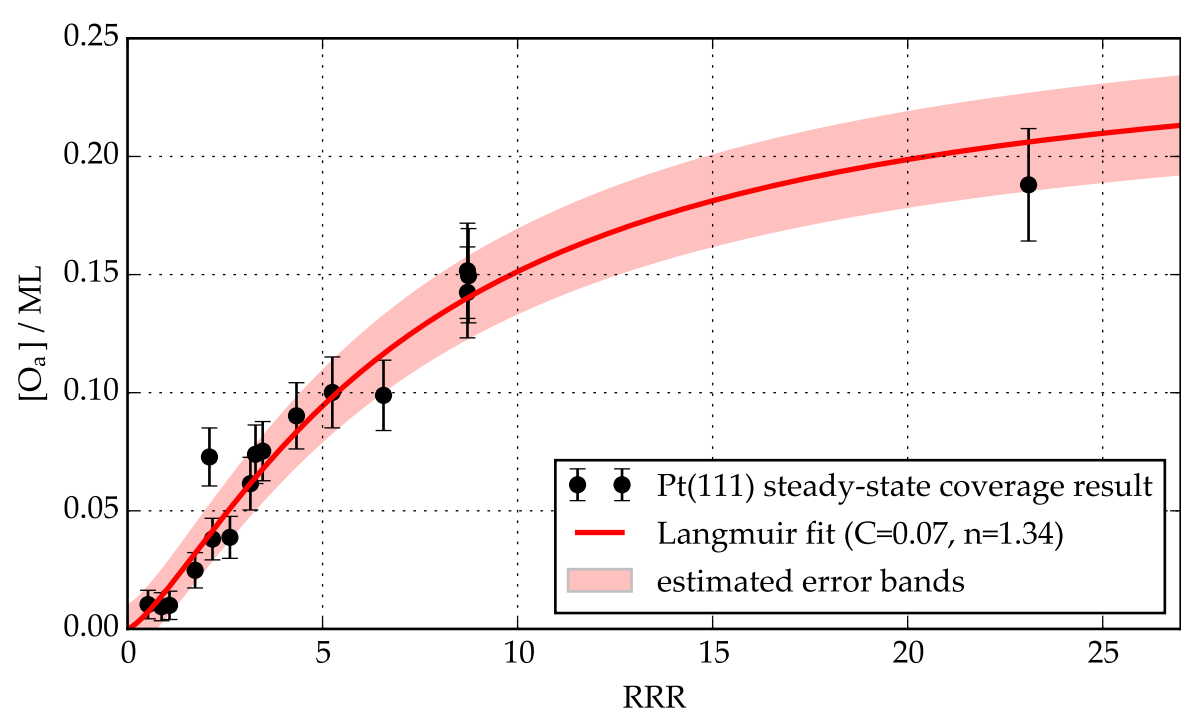

Figure 7.4: Result of several titrations on $\operatorname{Pt}(111)$ at $613 \mathrm{~K}$. The data can be described with the empirical function in eq. 7.25 , the fit parameters are given in the legend. The estimated error bands of the Langmuir fit are chosen to match the uncertainty of the titration data.

chosen to reflect the resulting uncertainty. The coverage as a function of RRR can be described using an empirically chosen function based on the Langmuir isotherm,

$$
\left[\mathrm{O}_{\mathrm{a}}\right]=\left[\mathrm{O}_{\mathrm{a}}\right]_{\max } \frac{\mathrm{C} \times \mathrm{RRR}^{\mathrm{n}}}{1+\mathrm{C} \times \mathrm{RRR}^{\mathrm{n}}} .
$$

The Langmuir isotherm is based on the Langmuir adsorption model which describes the adsorption of an ideal gas on a solid surface lattice with a defined number of binding sites. The resulting behavior of coverage (occupancy) vs partial pressure is expected to be closely related to the coverage vs RRR as measured in the titration experiment. In Eq. 7.25, the equilibrium constant of the Langmuir isotherm is replaced by a fit parameter $C$ and the partial pressure is replaced by RRR.

A fit to the $\operatorname{Pt}(111)$ titration data is shown as a red line and the associated error range is colored light red. The error range was chosen based on the same estimations as for the black closed circles described above. For $\operatorname{Pt}(111)$, where the step concentration $\theta_{s}$ is very small, the number of $\mathrm{O}$-atoms on terraces can be assumed to be equal to the total number of O-atoms determined from the titration, $\left[\mathrm{O}_{\mathrm{t}}\right] \approx\left[\mathrm{O}_{\mathrm{a}}\right]$. The initial concentration $\left[\mathrm{CO}_{t}\right]$ for the kinetic model on $\mathrm{Pt}(111)$ at any RRR can now be calculated using the fit function in eq. 7.25 as plotted in figure 7.4 and a lower and upper limit can be calculated using the error bands. The equilibrium $\mathrm{O}$-atom coverage was assumed to be independent of temperature.

Similar results for $\operatorname{Pt}(332)$ are shown in figure 7.5. Titrations were done at $573 \mathrm{~K}$ and $593 \mathrm{~K}$ to investigate the temperature dependence - 
A temperature dependence can be expected if the oxidation probability, i.e. the ratio of $\mathrm{CO}$ desorption vs reaction, changes strongly with temperature. No dependence is expected for a reaction probability close to unity. The desorption becomes a competitive channel at around $700 \mathrm{~K}$. no significant differences were found. This suggests that the titrations carried out at a single temperature for $\mathrm{Pt}(111)$ are sufficient.

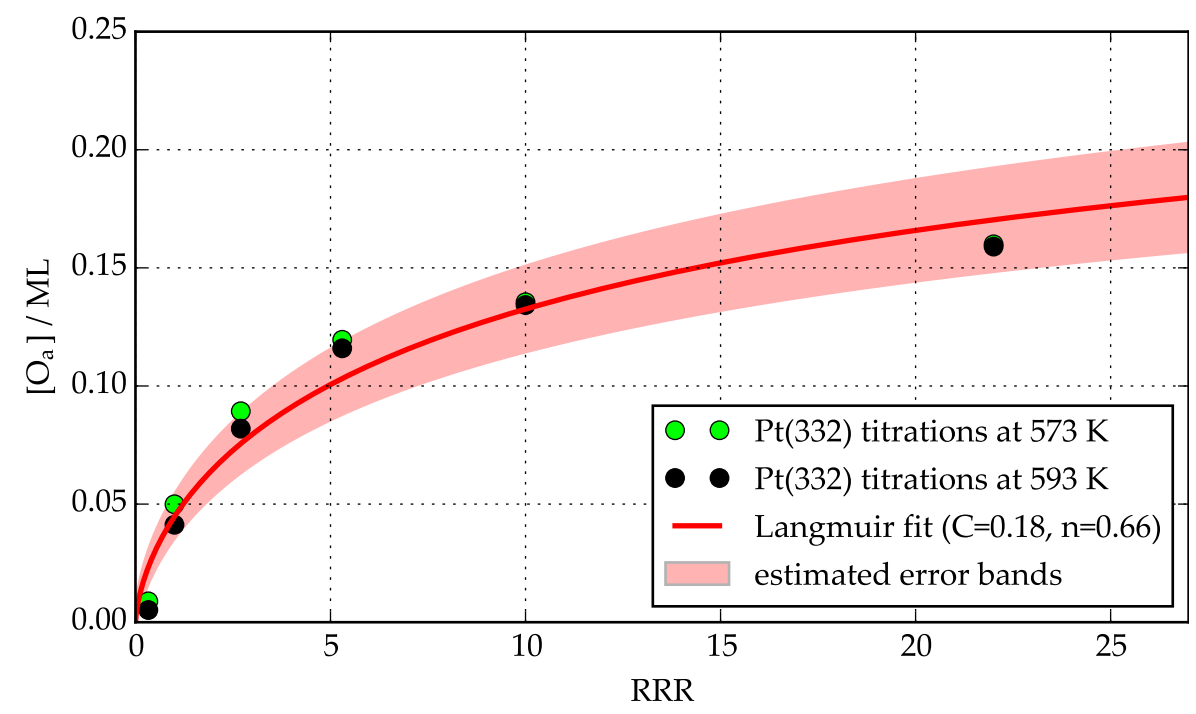

Figure 7.5: Titration result, i. e. the steady-state oxygen coverage on $\operatorname{Pt}(332)$ at $573 \mathrm{~K}$ and $593 \mathrm{~K}$. No significant temperature dependence was found. No error bars were estimated for the data points. A fit of the empirical Langmuir-type function from eq. 7.25 with estimated error bars is shown in red. The fit parameters are given in the legend.

On $\mathrm{Pt}(332)$, the step concentration $\theta_{s}$ is large enough to accommodate a significant fraction of the $\mathrm{O}$-atoms, even at higher total $\mathrm{O}$-atom coverage. The total concentration of adsorbed oxygen $\left[\mathrm{O}_{\mathrm{a}}\right]$ determined in the titration is equal the sum of the concentration of O-atoms on terraces and steps, $\left[\mathrm{O}_{\mathrm{t}}\right]+\left[\mathrm{O}_{\mathrm{s}}\right]=\left[\mathrm{O}_{\mathrm{a}}\right]$. Further information about the distribution at our experimental conditions is required to determine the partitioning of adsorbed $\mathrm{O}_{a}$ into these two species. A method to calculate the distribution at thermal equilibrium is presented in section $7 \cdot 3$.

\subsection{DISTRIBUTION OF ADSORBATES BETWEEN TERRACE AND STEP SITES}

\subsubsection{Equilibrium parameters from the canonical partition function}

The equilibrium concentration of $\mathrm{O}$-atoms on steps and terraces is defined by the difference in binding energy and the entropy of the system,

$$
k_{e q}=\exp \left(-\frac{\Delta G}{k_{B} T}\right),
$$

with the Gibbs free energy $\Delta \mathrm{G}=\Delta \mathrm{H}-\mathrm{T} \Delta \mathrm{S}$. At low temperatures, the entropy term is small and the molecules prefer the stronger bind- 
ing energy at step sites. At higher temperatures, the entropy term becomes more important. Due to the abundance of terrace sites, the entropy gain for $\mathrm{O}$-atoms on terrace sites is larger. The equilibrium partitioning of the total oxygen coverage can be quantitatively estimated from the canonical partition function for this system.

When distributing $n_{O}$ oxygen atoms over $n_{\text {step }}$ step and $n_{\text {terr }}$ terrace sites, $i$ atoms can be placed on step sites and $\left(n_{O}-i\right)$ atoms on terrace sites. For each combination of $i$ and $\left(n_{O}-i\right)$ the degeneracy is calculated as

$$
g_{i}=\left(\begin{array}{c}
n_{\text {step }} \\
i
\end{array}\right)\left(\begin{array}{c}
n_{\text {terr }} \\
n_{O}-i
\end{array}\right) .
$$

The total number of combinations is obtained by summing over $i$,

$$
g=\sum_{i}^{n_{O}} g_{i}=\sum_{i}^{n_{O}}\left(\begin{array}{c}
n_{\text {step }} \\
i
\end{array}\right)\left(\begin{array}{c}
n_{\text {terr }} \\
n_{O}-i
\end{array}\right)
$$

For a binding energy difference between $O_{s}$ and $O_{t}, \Delta E=E_{t}-E_{s}$, we calculate the respective probability of the terms of the sum as

$$
p_{B, i}=\exp \left(\frac{-E_{i}}{k_{B} T}\right)=\exp \left(\frac{-\Delta E\left(n_{O}-i\right)}{k_{B} T}\right) .
$$

This equation is valid for $n_{O}<n_{\text {step }}$ and $n_{O}<n_{\text {terr. }}$. If $n_{O}$ is larger than $n_{\text {step }}$, even at the lowest possible configuration some $\mathrm{O}$ atoms are on terraces. In this case we have to correct the energy term in equation 7.29 by the ground state energy of the system, $E_{0}=\left(n_{O}-\right.$ $\left.n_{\text {step }}\right) \Delta E$, as

$$
p_{B, i}=\exp \left(\frac{-E_{i}}{k_{B} T}\right)=\exp \left(\frac{-\left(\Delta E\left(n_{O}-i\right)-E_{0}\right)}{k_{B} T}\right) .
$$

The resulting canonical partition function is

$$
Q=\sum_{i}^{n_{O}} g_{i} \cdot p_{B, i}=\sum_{i}^{n_{O}}\left(\begin{array}{c}
n_{\text {step }} \\
i
\end{array}\right)\left(\begin{array}{c}
n_{\text {terr }} \\
n_{O}-i
\end{array}\right) \exp \left(\frac{-\Delta E\left(n_{O}-i\right)+E_{0}}{k_{B} T}\right)
$$

From the partition function we calculate the average energy of the system as

$$
\langle E\rangle=-\frac{\partial \ln Q}{\partial \beta} .
$$

with $\beta=\left(k_{B} T\right)^{-1}$. Instead of differentiating $\ln Q$ with respect to the reciprocal thermodynamic temperature $\beta$ we expand the fraction in Eq. 7.32 by $\frac{\partial T}{\partial T}$ and substitute

$$
\frac{\partial \beta}{\partial T}=\frac{\partial\left(k_{B} T\right)^{-1}}{\partial T}=-\frac{1}{k_{B} T^{2}} .
$$

Lowest possible configuration means lowest enthalpy possible. 
Reminder: The concentrations in square brackets are defined in $M L$ with respect to the entire surface.
The average energy from equation 7.32 becomes

$$
\langle E\rangle=k_{B} T^{2} \frac{\partial \ln Q}{\partial T} .
$$

By dividing the average energy plus the ground state energy of the system by the number of $\mathrm{O}$-atoms times the energy difference between terrace and step sites, we calculate the fraction of $\mathrm{O}$-atoms on terrace sites as

$$
\frac{\mathrm{O}_{\mathrm{t}}}{\mathrm{O}_{\mathrm{t}}+\mathrm{O}_{\mathrm{s}}}=\frac{\mathrm{n}_{\mathrm{O}}-\mathrm{i}}{\mathrm{n}_{\mathrm{O}}}=\frac{\langle\mathrm{E}\rangle+\mathrm{E}_{0}}{n_{\mathrm{O}} \Delta \mathrm{E}} .
$$

Using the equations above we can calculate the step occupancy $\theta_{\mathrm{O}} \mathrm{s}$ as a function of the total oxygen concentration $\left[\mathrm{O}_{a}\right]$ on the surface. The occupancy is zero when all sites are free and one if the steps are fully covered with O-atoms. Figure 7.6 shows a comparison for various binding energy differences and two extreme cases (step-first limit and no-preference limit) for $\mathrm{Pt}(111)$ and $\mathrm{Pt}(332)$.

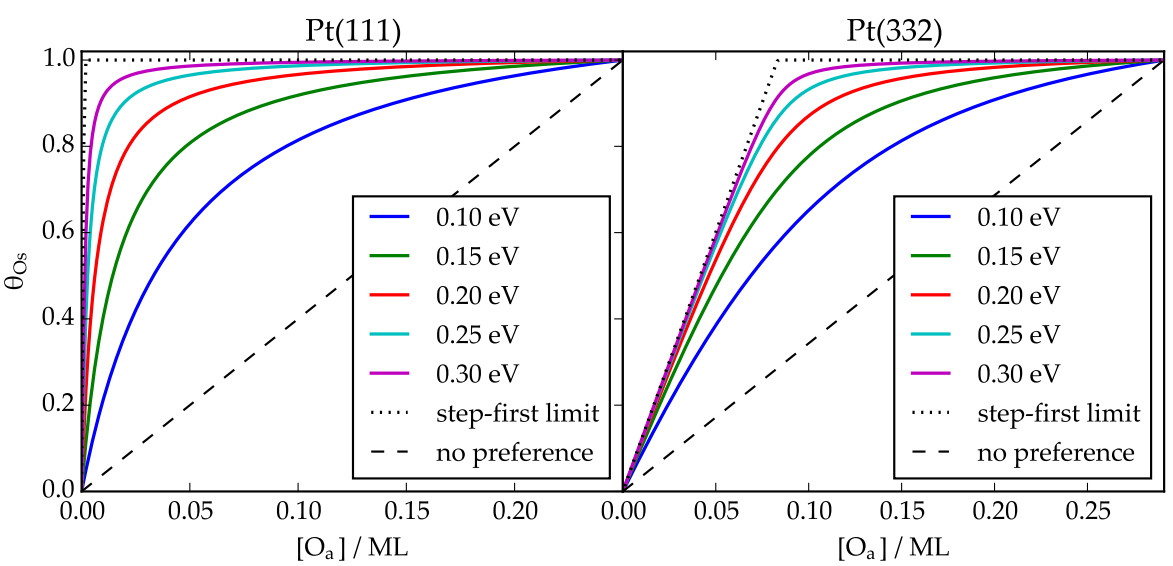

Figure 7.6: Step occupancy $\theta_{\mathrm{O} s}$ vs total oxygen coverage $\left[\mathrm{O}_{\mathrm{a}}\right]$ on $\mathrm{Pt}(111)$ (left) and on $\mathrm{Pt}(332)$ (right) at $613 \mathrm{~K}$. The binding energy differences between terrace and step sites is given in the legend. The maximum concentration of $\mathrm{O}$-atoms on steps is $0.00125 \mathrm{ML}$ on $\mathrm{Pt}(111)$ and 0.0833 ML on $\mathrm{Pt}(332)$.

Compared to the no-preference limit (dashed black line) the $\mathrm{O}$ atoms show a strong preference for step sites at $613 \mathrm{~K}$. A distribution entirely governed by entropy would behave like the no-preference limit. With larger binding energy difference the preference for steps increases: For a difference of $\Delta \mathrm{E}=0.25 \mathrm{eV}$ nearly all O-atoms bind to step sites until the step sites approach full occupation. In the stepfirst limit this point is reached at $\left[\mathrm{O}_{\mathrm{a}}\right]=0.00125 \mathrm{ML}$ on $\mathrm{Pt}(111)$ and at $0.0833 \mathrm{ML}$ on $\mathrm{Pt}(332)$.

We can also use the equations above to calculate the fraction of $\mathrm{O}$-atoms on terrace and step sites as a function of temperature. Figure 7.7 shows the step and terrace occupancy at a total coverage of $\left[\mathrm{O}_{\mathrm{a}}\right]=0.0005 \mathrm{ML}$. At low temperatures the entropy term is small 


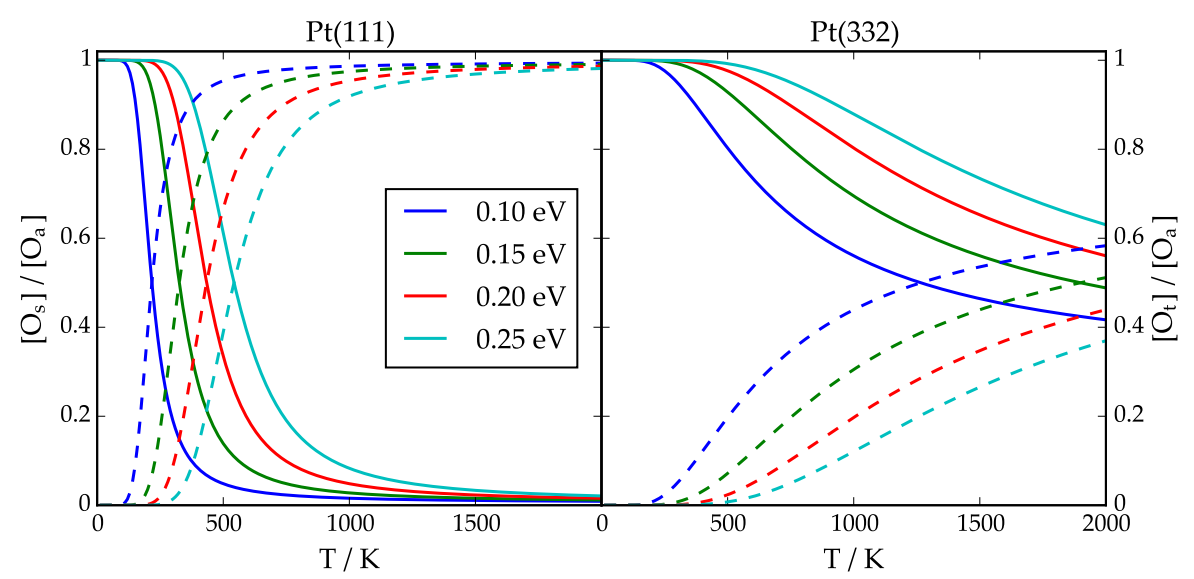

Figure 7.7: Fraction of O-atoms on steps $\left[\mathrm{O}_{s}\right] /\left[\mathrm{O}_{\mathrm{a}}\right]$ (solid lines, left y-axis) and fraction of O-atoms on terraces $\left[\mathrm{O}_{t}\right] /\left[\mathrm{O}_{\mathrm{a}}\right]$ (dashed lines, right y-axis) vs temperature. The calculation was done at low coverages of $\left[\mathrm{O}_{a}\right]=0.0005 \mathrm{ML}$, where saturation effects do not influence the result. The fraction on steps is one at low temperatures and decreases at higher temperatures. At very high temperatures the ratio $\left[\mathrm{O}_{s}\right]$ to $\left[\mathrm{O}_{\mathrm{t}}\right]$ approaches the abundance ratio of the respective sites, see text.

and the enthalpy term dominates. All O-atoms are on step sites. In the high temperature limit the entropy term shifts the equilibrium to an equal distribution of $\mathrm{O}$-atoms over terrace and step sites, weighted by their respective abundance. On $\mathrm{Pt}(111)$ this leads to a strong terrace preference. Note, however, that the fraction on steps does not go to zero. On $\mathrm{Pt}(332)$, where the numbers of terrace and step sites have roughly the same magnitude, the $\mathrm{O}$-atoms are distributed more equally at higher temperatures.

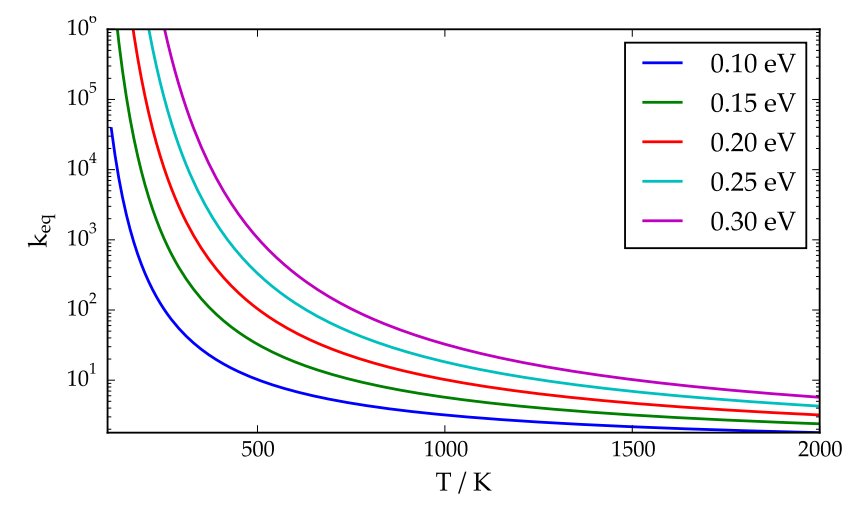

As oxygen can occupy every second step site but only every fourth terrace site, the high temperature terrace to step ratio on (332) is 0.83 to 0.33 .

Figure 7.8: Equilibrium constant $k_{e q}$ vs temperature: $k_{e q}$ is identical for $\operatorname{Pt}(111)$ and $\operatorname{Pt}(332)$.

This behavior is confirmed by the $k_{e q}$ vs $T$ plot in figure 7.8. At high temperatures the equilibrium constant approaches values of one. For surfaces with equal amounts of terrace and step sites this would lead to an equal distribution of $\mathrm{O}$-atoms, as can be infered from the oc- 
cupancies on $\operatorname{Pt}(332)$ in the right part of figure 7.7. On $\operatorname{Pt}(111)$, where the number of terrace sites is much larger, this leads to a strong terrace preference at high temperatures.

\subsubsection{Titration combined with partition function}

We use these equilibrium calculations to provide an initial concentration of $\left[\mathrm{O}_{s}\right]$ and $\left[\mathrm{O}_{t}\right]$ for the kinetic model. The binding energy diference between terrace and step sites was chosen to match the $0.26 \mathrm{eV}$ value reported Morimoto et al. [59, 60]. The result of combining the titration curve $\left(\left[\mathrm{O}_{a}\right]\right.$ vs $\left.\mathrm{RRR}\right)$ with the equilibrium calculation for $\mathrm{Pt}(111)$ is shown in figure 7.9. The total amount of O-atoms on the

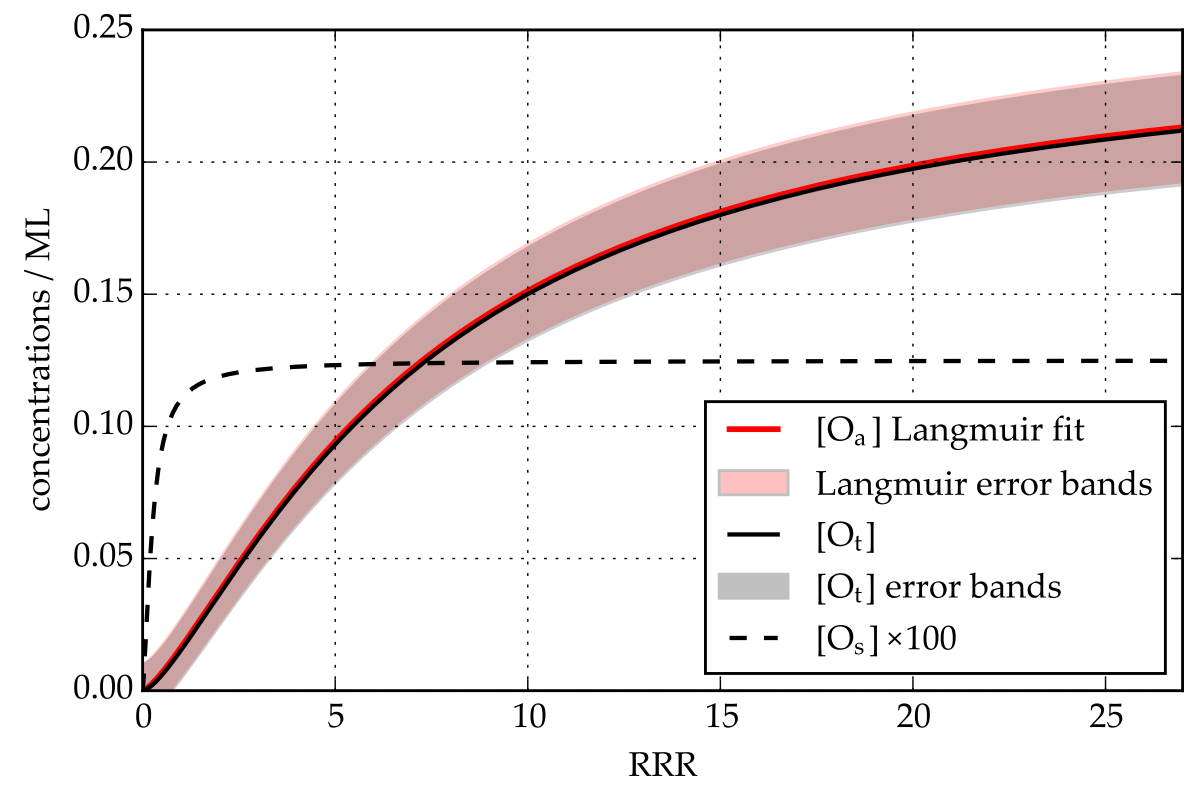

Figure 7.9: Equilibrium coverages vs $R R R$ on $\operatorname{Pt}(111)$ : The Langmuir fit with error bands from figure 7.4 is plotted as a red line and red shaded area. The concentration of $\mathrm{O}$-atoms on terrace sites $\left[\mathrm{O}_{\mathrm{t}}\right]$ is plotted as a black line with similar error bands. The concentration of O-atoms on steps (black, dashed line) has been multiplied by 100 for better visibility.

Note that the concentration on steps was multiplied by 100 to be visible in figure 7.9 .

Reminder: Every second step site can be occupied by oxygen. surface $\left[\mathrm{O}_{a}\right]$ as predicted by the titration is partitioned into $\left[\mathrm{O}_{t}\right]$ and $\left[\mathrm{O}_{s}\right]$ according to the equilibrium calculation. Because the number of step sites on $\operatorname{Pt}(111)$ is very small, most of the O-atoms on $\mathrm{Pt}(111)$ are on terrace sites. Thus the respective concentrations (red and black line) closely follow each other. The error bands for $\left[\mathrm{O}_{t}\right]$ were used as boundaries when fitting the kinetic model or more specifically the $\left[\mathrm{O}_{t}\right]$ concentration to the data. Because the error bands of $\left[\mathrm{O}_{\mathrm{a}}\right]$ are much larger than the maximum possible amount of $\mathrm{O}$-atoms on steps, no boundaries were used when fitting $\left[\mathrm{O}_{s}\right]$ except restricting the value to be between zero and half the step density. 
The combination of titration data with equilibrium calculations for $\mathrm{Pt}(332)$ is shown in figure 7.10. Since the step density is larger, the

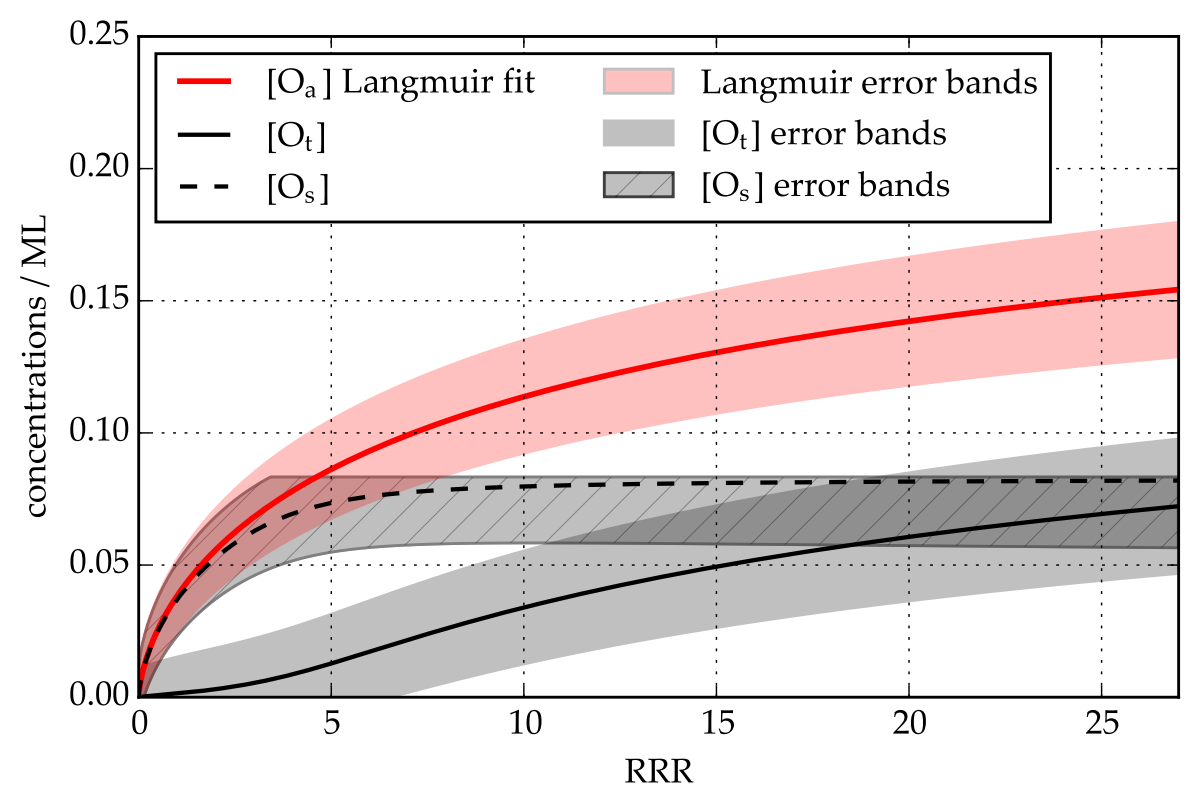

Figure 7.10: Equilibrium coverages vs $R R R$ on $\mathrm{Pt}(332)$ : The Langmuir fit with error bands from figure 7.5 is plotted in red. The concentrations of $\mathrm{O}$-atoms on terrace and step sites are calculated as described in the text. The error bands from the Langmuir fit are applied to both, except that the error bands for $\left[\mathrm{O}_{s}\right]$ are cut off at the maximum value of $\left[\mathrm{O}_{s}\right]=0.0833$.

concentration of $\mathrm{O}$-atoms on steps $\left[\mathrm{O}_{s}\right]$ (black, dashed line) can also reach larger values and is not multiplied by any factor. The same error range obtained from the Langmuir fit is applied to both $\mathrm{O}$ atom species. The error band for $\left[\mathrm{O}_{s}\right]$ is cut off at the upper limit of 0.083 ML. At this value every second step site on a crystal with a step concentration of $0.166 \mathrm{ML}$ is occupied. 
This section describes the input values for the Pt(111) simulation. The input values for Pt(332) are obtained analogously.

The effective diffusion rate coefficient (conversion rate coefficient) is further discussed in section 8.1.4.

\subsection{RESULT OF THE KINETIC MODEL}

\subsubsection{Input values for the numeric solution}

As described in section 7.1.3 the numeric approximation to the differential equations is a solution to an initial-value problem. Both initial concentrations and rate constants must be provided to solve it.

The input for the steady-state oxygen concentrations were obtained as described in section 7.2 and 7.3. The initial concentrations of $\left[\mathrm{CO}_{t}\right]$ and $\left[\mathrm{CO}_{s}\right]$ before arrival of the $\mathrm{CO}$ beam pulse are zero due to the high reaction and desorption rates at the temperatures of the experiment and the minimum time of $4 \mathrm{~ms}$ between $\mathrm{CO}$ pulses. Consequently, the free terrace and step sites depend only on the number of O-atoms on the surface. An example set of input concentrations is shown in table 7.1 on the left.

Some of the rate constants, $k_{d}^{t}$ and $k_{d}^{s}$, are known from previous experiments. The effective diffusion rate coefficient for $\mathrm{CO}$ from terrace to step sites was estimated. A value of $0.3 \mathrm{eV}$, which was the result several previous optimizations converged on, was chosen for the activation energy. This agrees well with the value of $0.3 \mathrm{eV}$ reported by Poelsema et al. [71].

In our kinetic model the lifetime of the hyperthermal channel is 1 over the sum of all rate coefficients depleting it, $\tau=1 / \sum_{i} k_{i}$, where $k_{i}$ are rate coefficients of first-order or pseudo first-order processes. The observed hyperthermal channel lifetime between 20 and $200 \mu s$ sets an upper limit on each individual rate coefficient. Since the effective diffusion rate constant cannot be greater than this sum, we calculated an upper limit of $10^{11} \mathrm{~s}^{-1}$ to $10^{10} \mathrm{~s}^{-1}$ for the prefactor of the diffusion rate coefficient. A first guess for the reaction rate coefficients was supplied through the trial and error method. A set of rate coefficient is shown on the right in table 7.I.

The concentrations were then used to construct the numeric model, as can be seen in the top panel of figure 7.11 at $t=0 \mu$ s.

The activation energies and prefactors were used to calculate the rate constants at a given temperature, in this example at $603 \mathrm{~K}$. The rate constants were plugged into the differential equations, and the system was solved, yielding the concentrations at each time step. This numeric solution is plotted in figure 7.11 as a function of time. The two top panels show the concentrations on the surface, on a logscale and on a linear scale, and the bottom panel shows the $\mathrm{CO}_{2}$ flux of the hyperthermal and thermal channels.

The concentrations $\left[\mathrm{CO}_{t}\right]$ and $\left[\mathrm{CO}_{s}\right]$ are zero at the beginning and the system is at equilibrium. After a few hundred $\mu$ s the concentrations $\left[\mathrm{CO}_{\mathrm{t}}\right]$ and $\left[\mathrm{CO}_{s}\right]$ are perturbed by the adsorption of $\mathrm{CO}$ from the molecular beam. This is the inhomogeneous part of the differential equations. The initial rise in concentration of $\left[\mathrm{CO}_{t}\right]$ on the surface 

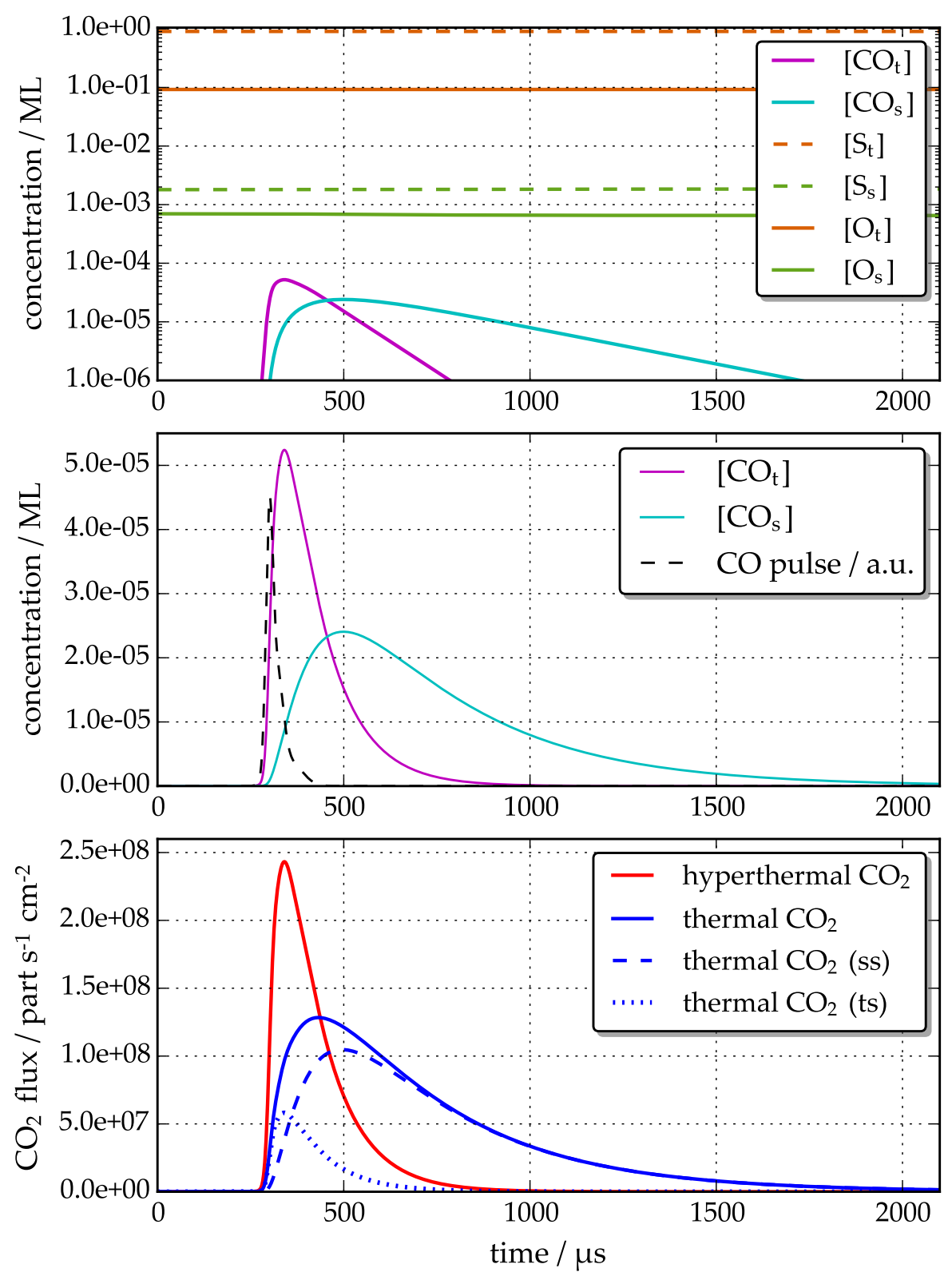

Figure 7.11: Numeric solution output: The upper two panels show the concentrations on the surface as a function of time on a log- and on a linear scale, for the reaction of $\mathrm{CO}$ on an oxygen pre-covered $\mathrm{Pt}(111)$ surface at $603 \mathrm{~K}$. The initial concentrations at $\mathrm{t}=\mathrm{o} \mu \mathrm{s}$ in the top panel are as specified in table 7.1. The change in concentrations of free terrace sites $\left[S_{t}\right]$ and oxygen on terraces $\left[\mathrm{O}_{t}\right]$ due to the arrival of the $\mathrm{CO}$ pulse and subsequent reactions is miniscule. The concentrations of free step sites $\left[S_{s}\right]$ and oxygen on steps $\left[\mathrm{O}_{s}\right]$ change by a larger relative amount, but the change is barely visible on a logscale. The dose of the CO pulse was $1.2 \times 10^{-4} \mathrm{ML}$ as estimated from the titration. The bottom panel shows the resulting $\mathrm{CO}_{2}$ flux. The hyperthermal $\mathrm{CO}_{2}$ flux (red) closely follows the concentration of $\left[\mathrm{CO}_{\mathrm{t}}\right]$ on the surface. The thermal $\mathrm{CO}_{2}$ flux is a sum of the ts-reaction (blue, dotted) with $\left[\mathrm{CO}_{\mathrm{t}}\right]$ and the ss-reaction (blue, dashed) with $\left[\mathrm{CO}_{s}\right]$. 
The change in concentration $d\left[\mathrm{CO}_{t}\right] / d t$ closely follows the CO beam flux at that time. The $\left[\mathrm{CO}_{t}\right]$ concentration (purple line) as a function of time is approximately equal to the integral of the CO pulse.

\begin{tabular}{lc|lcc}
\hline \multicolumn{2}{c|}{ CONCENTRATION / ML } & RATE COEFFICIENTS & $\mathrm{E}_{\mathrm{a}} / \mathrm{eV}$ & $\mathrm{A} / \mathrm{s}^{-1}$ \\
\hline$\left[\mathrm{CO}_{\mathrm{t}}\right]$ & $\mathrm{O}$ & $\mathrm{k}_{\mathrm{d}}^{\mathrm{t}}$ & 1.29 & $5.9 \times 10^{13}$ \\
{$\left[\mathrm{CO}_{\mathrm{s}}\right]$} & $\mathrm{O}$ & $\mathrm{k}_{\mathrm{d}}^{\mathrm{s}}$ & 1.28 & $5.0 \times 10^{12}$ \\
{$\left[\mathrm{O}_{\mathrm{t}}\right]$} & 0.092 & $\mathrm{k}_{\mathrm{diff}}^{\mathrm{ts}}$ & 0.3 & $2.1 \times 10^{6}$ \\
{$\left[\mathrm{O}_{\mathrm{s}}\right]$} & 0.0007 & $\mathrm{k}_{\mathrm{r}}^{\mathrm{tt}}$ & 0.6 & $3.5 \times 10^{9}$ \\
{$\left[\mathrm{~S}_{\mathrm{t}}\right]$} & 0.9055 & $\mathrm{k}_{\mathrm{r}}^{\mathrm{ts}}$ & 0.4 & $5.9 \times 10^{7}$ \\
{$\left[\mathrm{~S}_{\mathrm{s}}\right]$} & 0.0018 & $\mathrm{k}_{\mathrm{r}}^{\mathrm{ss}}$ & 0.65 & $2.9 \times 10^{9}$ \\
\hline
\end{tabular}

Table 7.1: Input values for the kinetic model. Left: Note that the sum of the free step site concentration $\left[\mathrm{S}_{s}\right]$ and oxygen on steps $\left[\mathrm{O}_{s}\right]$ yields the step concentration $\theta_{s}=0.0025 \mathrm{ML}$. Similarly, the terrace concentrations add up to $\theta_{t}=0.9975$ ML. Right: Any rate coefficients leading to lifetimes in the $\mu$ s range can be used as input to the kinetic model, but for simplicity the ones shown here are the result of the full optimization on $\mathrm{Pt}(111)$.

closely follows the shape of the molecular beam (dashed, black line) in the middle panel. The $\mathrm{CO}$ adsorbed to the surface immediately starts to desorb, diffuse and react according to the homogeneous part of the differential equations. The behaviour is qualitatively similar to the convolution of an exponential decay over the incident beam pulse, as done for trapping-desorption of $\mathrm{CO}$ in section 5.2.2.

The diffusion from terraces to steps increases $\left[\mathrm{CO}_{s}\right]$ - notice the delay in initial rise time between the purple and the cyan curves. Reaction of $\left[\mathrm{CO}_{\mathrm{t}}\right]$ with $\left[\mathrm{O}_{\mathrm{t}}\right]$ forms hyperthermal $\mathrm{CO}_{2}$, as shown as a red curve in the lower panel. The reaction of $\left[\mathrm{CO}_{t}\right]$ near step edges with oxygen atoms on steps forms thermal $\mathrm{CO}_{2}$ (blue, dotted curve) and the reaction of $\left[\mathrm{CO}_{s}\right]$ with $\left[\mathrm{O}_{s}\right]$ also forms thermal $\mathrm{CO}_{2}$ (blue, dashed curve). Notice how the contribution of this ss-reaction follows the concentration of $\left[\mathrm{CO}_{s}\right]$ (cyan curve) in the middle panel. The observed thermal $\mathrm{CO}_{2}$ is the sum of these two components.

The result of interest, i.e. the hyperthermal $\mathrm{CO}_{2}$ flux (red line) and the thermal $\mathrm{CO}_{2}$ flux (blue line), can now be compared to experimental kinetic traces. The residual at this set of parameters (rate coefficients and concentrations) is calculated for both channels. By iteratively varying the parameters and minimizing the residual, the numeric solution can now be fitted to the experimental data. 


\subsubsection{Python code structure}

As the Python code is quite complex, I give a brief overview how it works. A flow chart of the fit routine is shown in figure 7.12.

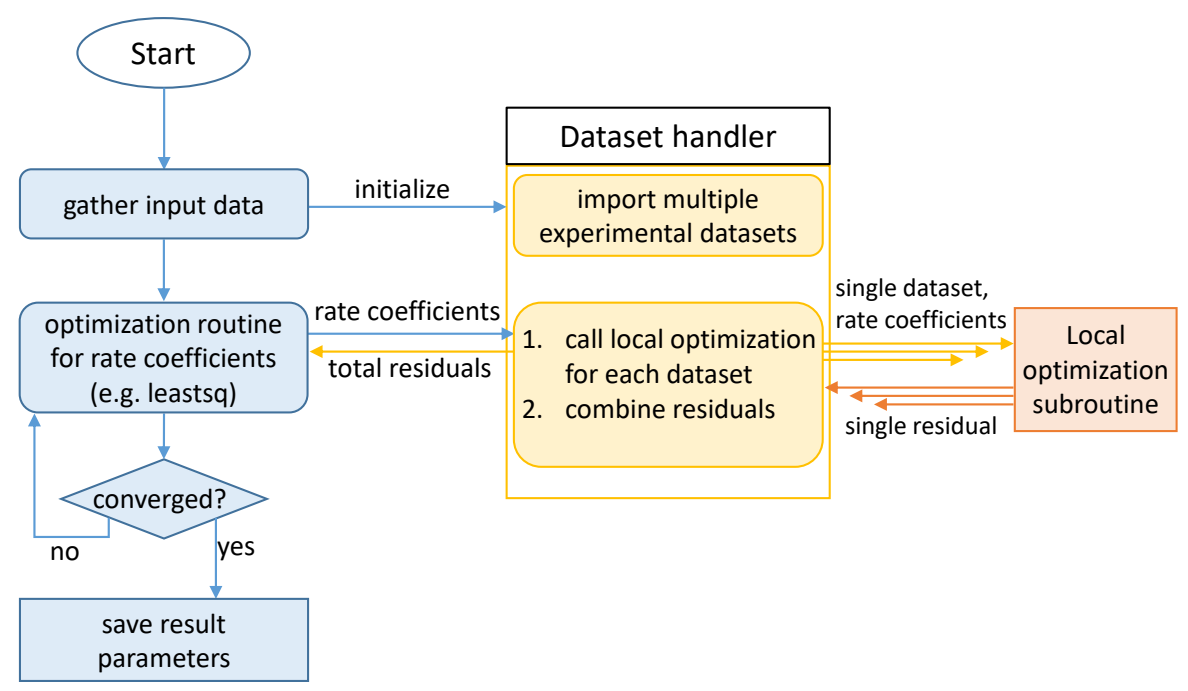

An introduction to the Python code structure might help future researchers at Beamer II adapt the code to their problem.

(a) Flow chart of the Python code

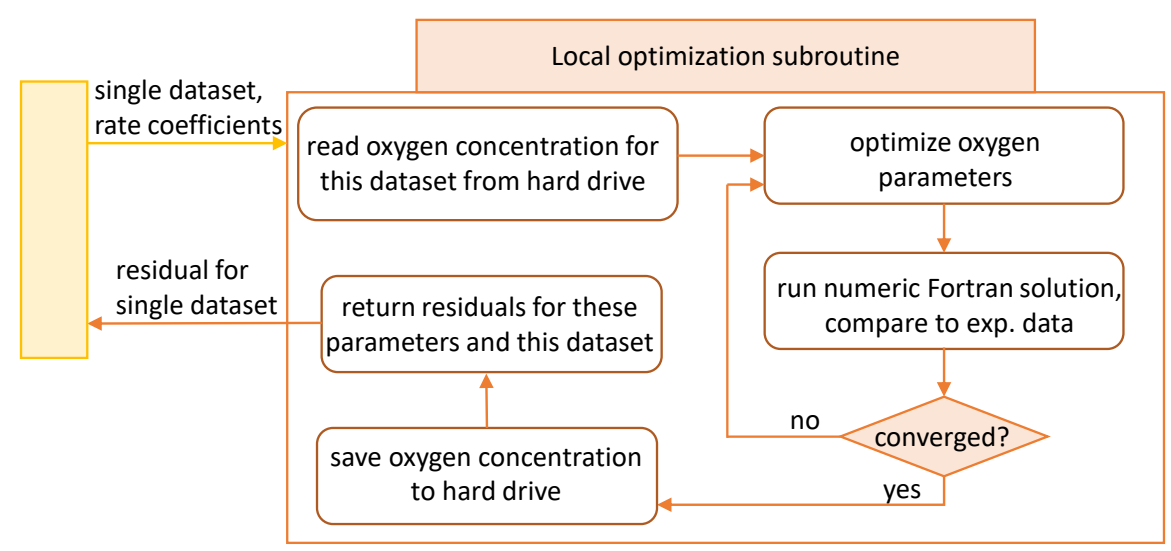

(b) Flow chart of the local optimization subroutine

Figure 7.12: Simplified Python flow chart.

The first step in the code is to define the location of the kinetic traces of the different measurements. The complete dataset presented in this thesis consists of 126 measurements at different experimental conditions, i.e. RRR and temperature. For each measurement the thermal and hyperthermal kinetic traces were extracted before executing the code. All kinetic traces were time-of-flight shifted and the flux was properly scaled to represent the relative total flux. This is summarized as "gather input data" in the top left of panel (a). Along with a file defining the molecular beam pulse shape, all input data is passed to the "dataset handler". The dataset handler imports all Scaling: represent the relative flux seen in speed and angular distribution. experimental data. 
${ }^{*}$ Lowest possible under the assumption that the subroutine found the global minimum.

The temperature range investigated was $563-$ $623 \mathrm{~K}\left(290-350^{\circ} \mathrm{C}\right)$.

R-squared values of 1 mean that all variance of the data is explained by the model,

$R$-squared values

of 0 mean that the model doesn't fit at all.
Next, an initial guess for the reaction rate coefficients is provided and passed to a global optimization routine (blue box). The optimization routine varies the rate coefficients and calls a function of the dataset handler (yellow box), which will eventually return the lowest possible residual for this set of rate coefficients.

To do this, the dataset handler executes a "local optimization subroutine" (red box, further specified in panel (b)) for each reaction condition of the dataset and the current set of rate coefficients. The local subroutine optimizes the oxygen concentration on terraces and steps within specified boundaries and returns the residual of the kinetic traces at this reaction condition. The dataset handler collects all residuals and returns them to the global optimization routine (blue box).

With 126 experimental measurements and about 200 iterations per local optimization, the numeric solution needs to be calculated about 25 ooo times per global optimization step. Since these calls are parallelized and computationally optimized (90\% of the time is spent at the Fortran solver), one global step is calculated in 10-30 s on a quad-core i7 computer.

\subsubsection{Fit result on $P t(111)$}

The result of the numeric solution is compared to a few experimental kinetic traces in figure 7.13. The traces selected here span the whole coverage range investigated at the middle temperature of $593 \mathrm{~K}$. The comparison of all kinetic traces at all temperatures to the numeric solution, a total of 252 thermal and hyperthermal traces, is shown in figure A.I in the appendix. The fit visually agrees well across all RRRs and temperatures. One way to assess the goodness of fit is R-squared,

$$
R^{2}=1-\frac{\sum_{i}\left(y_{i}-f_{i}\right)^{2}}{\sum_{i}\left(y_{i}-\bar{y}\right)^{2}}
$$

where $y_{i}$ are the experimental flux values at time $i, f_{i}$ the model values and $\bar{y}$ the mean flux values of a kinetic trace. R-squared is 1 minus the residual sum of squares over the total sum of squares. The mean R-squared across the thermal channel is 0.983 , the mean $\mathrm{R}$-squared across the hyperthermal channel is 0.939. This implies that the fit quality with respect to the thermal channel is slightly better.

For a more accurate assessment of the fit quality it is necessary to examine the residuals, which are shown as blue and red dots below each kinetic trace. The residuals of the hyperthermal channel show a systematic trend across some of the measurements - the experimental flux (red open circles in the upper panels) rises slightly earlier than the numeric result (red line) leading to positive residuals (red dots, lower panels). With increasing reaction time the experimental 

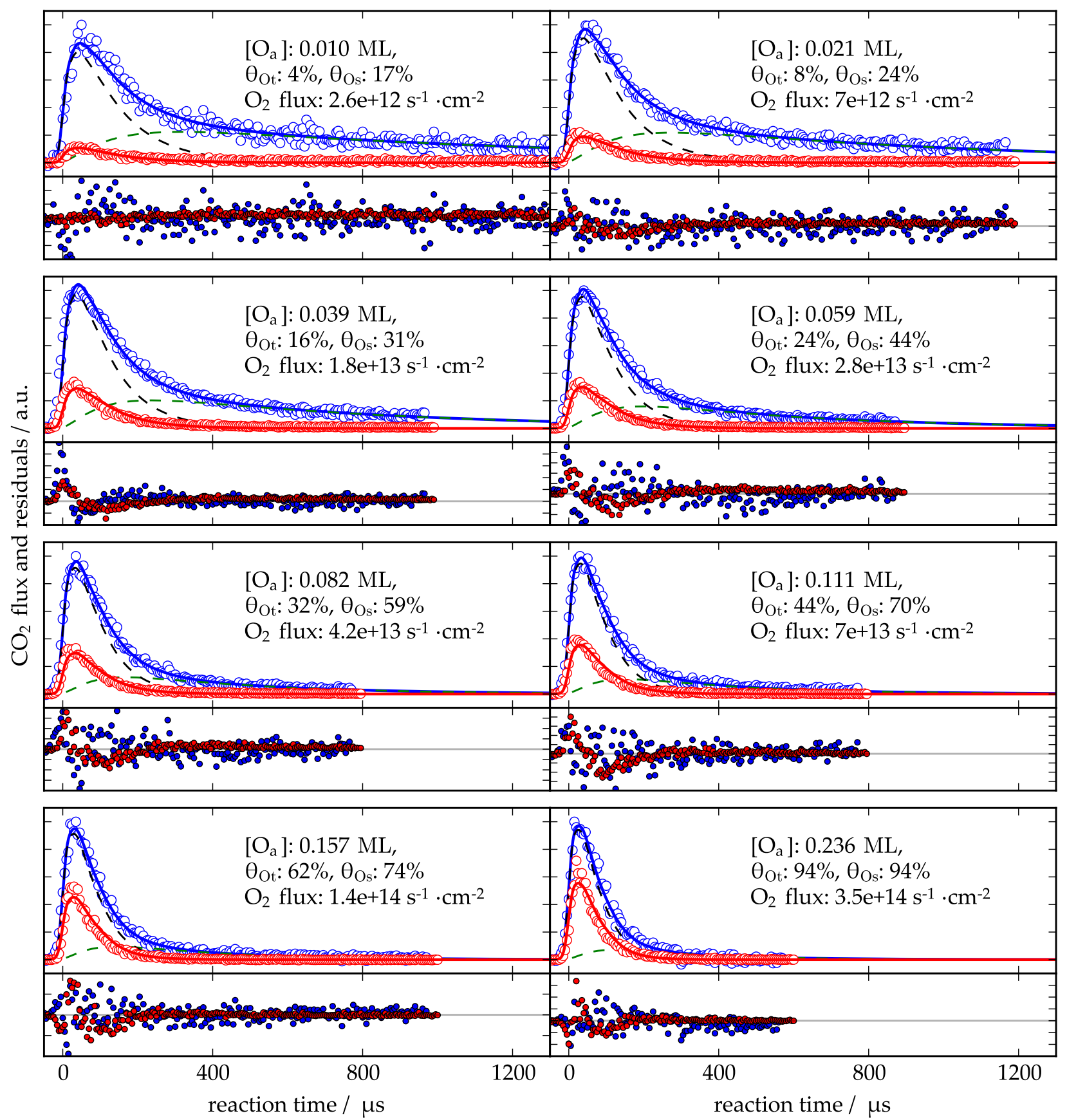

Figure 7.13: $\mathrm{Pt}(111)$ result at $593 \mathrm{~K}$ : The fit result is compared to the kinetic traces for several RRRs. The $\mathrm{O}_{2}$ and $\mathrm{CO}$ flux ratios, as given in the legend and in the respective inset, correspond to RRRs of $0.25,0.67,1.67,2.66,4.01,6.66,13.3$ and 33.3 (left to right, top to bottom). The hyperthermal channel is plotted in red (experimental data as open circles, numeric solution as solid curve) and the thermal channel is plotted in blue. The ts- and ss-reaction contribution to the thermal channel are plotted as black and green dashed curves. Below the kinetic traces the residuals for both channels are plotted as blue (thermal) and red dots (hyperthermal). The oxygen coverages and $\mathrm{O}_{2}$ fluxes are given as an inset. The timeaveraged $\mathrm{CO}$ flux was $2.2 \times 10^{12} \mathrm{~s}^{-1} \mathrm{~cm}^{2}$ for all measurements. 
flux drops slightly earlier than the numeric result. This can be an indication that the TOF of the hyperthermal channel has been overestimated. If the velocity is determined too small and thus the TOF too large, the experimental kinetic trace (red open circles) will be shifted too far to the left when converting the $\mathrm{x}$-axis from beam-laser delay to reaction time. Of course it is equally possible that both the thermal channel TOF and the incoming beam TOF are calculated slightly wrong. A deviation of $5 \mu \mathrm{s}$ is sufficient to cause a comparable mismatch. Since this is the same magnitude as the uncertainties of the different TOFs, no further steps were taken to improve the conversion from beam-laser delay to reaction time. The distribution of the thermal channel residuals around zero (grey line in the lower panels) is rather random, suggesting a good fit to the thermal channel. Regarding the fit quality, the kinetic model as expressed in the previous sections is suitable to reproduce the experimental data.

The rate coefficients for the fits to the $\mathrm{Pt}(111)$ data shown in figure 7.13 and A.1 are listed below in table 7.2.

Table 7.2: Fit result on $\operatorname{Pt}(111)$ - rate coefficients.

\begin{tabular}{c|ccc}
\hline & RATE COEFFICIENTS & $\mathrm{E}_{\mathrm{a}} / \mathrm{eV}$ & $\mathrm{A} / \mathrm{s}^{-1}$ \\
\hline input & $k_{\mathrm{d}}^{\mathrm{t}}$ & 1.29 & $5.9 \times 10^{13}$ \\
(constrained) & $\mathrm{k}_{\mathrm{d}}^{\mathrm{s}}$ & 1.28 & $5.0 \times 10^{12}$ \\
\hline \multirow{3}{*}{ result } & $\mathrm{k}_{\mathrm{diff}}^{\mathrm{ts}}$ & $0.3^{*}$ & $2.1_{-1.8}^{+13} \times 10^{6}$ \\
(floated) & $k_{\mathrm{r}}^{\mathrm{tt}}$ & $0.6 \pm 0.1$ & $3.5_{-3.0}^{+21} \times 10^{9}$ \\
& $k_{\mathrm{r}}^{\mathrm{ts}}$ & $0.4 \pm 0.1$ & $5.9_{-5.1}^{+36} \times 10^{7}$ \\
& $k_{\mathrm{r}}^{s s}$ & $0.65 \pm 0.1$ & $2.9_{-2.5}^{+18} \times 10^{9}$ \\
\hline
\end{tabular}

${ }^{*}: \mathrm{E}_{\mathrm{a}}$ for the effective diffusion rate coefficient was not varied.

In addition to the rate coefficients the fit was allowed to vary the coverage of $\mathrm{O}$-atoms on terraces and steps. The resulting $\mathrm{O}$-atom coverage is presented in section 7.5.2. The rate coefficients obtained from the kinetic model fit are discussed in section 8.1.5.

\subsubsection{Fit result on $\mathrm{Pt}(332)$}

The experimental data measured using the $\mathrm{Pt}(332)$ crystal has been fit using the same procedure. All comparisons of the kinetic model to the experimental data are shown in the appendix in figure A.2. An R-squared of 0.983 for the thermal and 0.936 for the hyperthermal channel suggests that the goodness of fit is similar to that on $\mathrm{Pt}(111)$. Careful examination of the kinetic traces revealed no significant systematic deviation. The rate coefficients obtained from the fit are listed below in table $7 \cdot 3$. 
Table 7.3: Fit result on $\operatorname{Pt}(332)$ - rate coefficients.

\begin{tabular}{c|ccc}
\hline & RATE COEFFICIENTS & $\mathrm{E}_{\mathrm{a}} / \mathrm{eV}$ & $\mathrm{A} / \mathrm{s}^{-1}$ \\
\hline input & $\mathrm{k}_{\mathrm{d}}^{\mathrm{t}}$ & 1.28 & $5.9 \times 10^{13}$ \\
(constrained) & $\mathrm{k}_{\mathrm{d}}^{\mathrm{s}}$ & 1.18 & $1.5 \times 10^{12}$ \\
\hline \multirow{2}{*}{ result } & $\mathrm{k}_{\mathrm{diff}}^{\mathrm{ts}}$ & $0.32^{*}$ & $1.5_{-1.3}^{+9.2} \times 10^{6}$ \\
(floated) & $\mathrm{k}_{\mathrm{r}}^{\mathrm{tt}}$ & $0.6 \pm 0.1$ & $3.8_{-3.3}^{+23} \times 10^{9}$ \\
& $\mathrm{k}_{\mathrm{r}}^{\mathrm{ts}}$ & $0.4 \pm 0.1$ & $4.1_{-3.5}^{+25} \times 10^{7}$ \\
& $\mathrm{k}_{\mathrm{r}}^{\mathrm{ss}}$ & $0.65 \pm 0.1$ & $2.6_{-2.2}^{+16} \times 10^{9}$ \\
\hline
\end{tabular}

${ }^{*}: \mathrm{E}_{\mathrm{a}}$ for the effective diffusion rate coefficient was not varied.

The desorption rate constant from steps differs from the one used in the $\operatorname{Pt}(111)$ calculation. The value used in the $\operatorname{Pt}(111)$ calculation was extracted from a single experimental dataset for $\mathrm{CO}$ trappingdesorption on $\operatorname{Pt}(332)$, while the value in the $\operatorname{Pt}(332)$ calculation used several datasets. While the Arrhenius parameters differ from each other, the value of the resulting rate constant is very comparable.

At a first glance the resulting reaction rate coefficients on $\mathrm{Pt}(332)$ are very close to the fit result on $\mathrm{Pt}(111)$, the largest deviation is a factor of 1.5 on the prefactor of the ts-reaction. The results are discussed in section 8.1.5.

\subsection{SELF-CONSISTENCY CHECKS}

\subsubsection{Sensitivity analysis}

In order to identify cross-correlations when fitting the different reaction rate coefficients, a sensitivity analysis was required. One possible approach is to vary two activation energies at the same time and examine the resulting fit quality (total fit residual) in a $2 \mathrm{D}$ plot (contour plot).

The residual for a single measurement,

$$
\hat{\epsilon}_{\text {single }}=\sum_{i}\left|y_{i}^{M B}-f_{i}^{M B}\right|+\sum_{i}\left|y_{i}^{H T}-f_{i}^{H T}\right|,
$$

is the sum of residuals of the thermal (MB) channel plus the sum of residuals of the hyperthermal (HT) channel, with y denoting the experimental data and $f$ the numeric model data at each point $i$. The residuals are similar to the ones shown below the kinetic traces in figure 7.13. The total fit residual is then calculated by adding the single residuals $\hat{\epsilon}_{\text {single }}$ of all 126 measurements, i.e. 126 different combinations of temperature and RRR.

To generate the contour map the activation energies were varied in a range of $0.2 \mathrm{eV}$ around the optimum fit value as presented in

A different approach would be to use the covariance matrix result of the leastsquare fit routine. The resulting errors were unreasonably low, on the range of $0.1 \%$. I could not find a reason for this behaviour or solve the underlying problem, so I decided to estimate the uncertainty from a sensitivity analysis. 
Information on how to separately vary activation energy and pre-exponential factor can be found in the appendix in section B.2. table 7.2 , with a total of 31 different values per activation energy. The total residual was then calculated for every pair in the resulting $31 \times$ 31 matrix.

Figure 7.14 shows three different contour plots calculated with the $\operatorname{Pt}(111)$ data. The upper panel (a) shows the effect of varying the activation energies for the tt-reaction (y-axis) and the ts-reaction (x-axis). The middle panel (b) varies the activation energy for the ts-reaction (y-axis) and the ss-reaction (x-axis) and the lower panel (c) shows the contour plot for the tt-ss pair.

The most prominent feature in the two upper panels including $E_{a}^{t s}$ is the strong gradient of the total residual along the $E_{a}^{t s}$-axis. The gradient along the other two axes is smaller, resulting in an oval shape of the contour lines. The total residual is very sensitive to changes of the activation energy of the ts-reaction. This suggests, that $E_{a}^{t s}$ has the smallest uncertainty of the three activation energies.

Cross-correlations between parameters appear as slanted shapes in contour plots. The ellipse in panel (b) is slightly slanted, with a smaller gradient along the top-left to bottom-right diagonal compared to the other diagonal. A small diagonal gradient indicates that the changes in activation energies partly compensate each other, while a large gradient indicates a multiplicatively increased residual.

The bottom panel (c) shows the residual as a function of the tt- and ss-reaction. The gradient along the two axes is equally large, implying equal sensitivity of the fit quality on the two parameters. The gradients along the two diagonals are also comparable, which suggests little cross-correlation between the two parameters. Together this results in more or less circular contour lines. Note that the distance between the contour lines in the lower panel is smaller than in the other panels. 


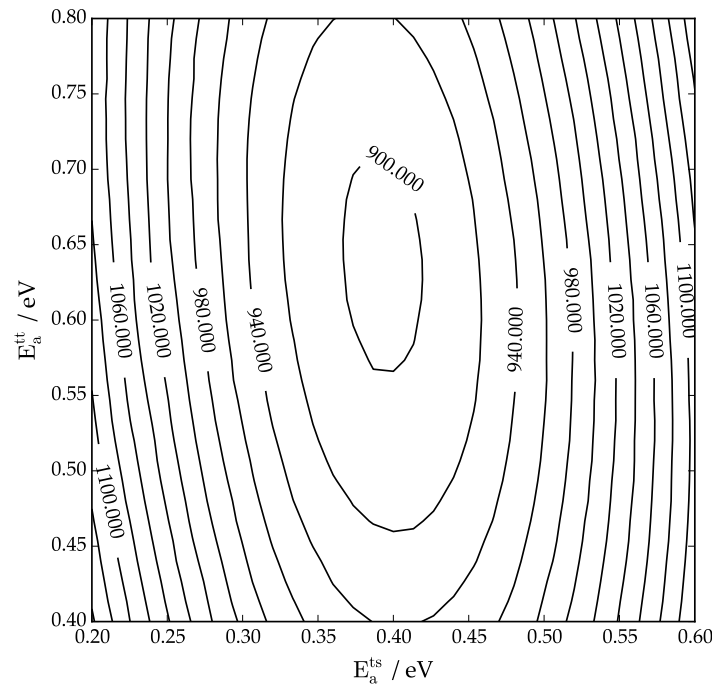

(a) Residual vs activation energy (tt- and tsreaction)

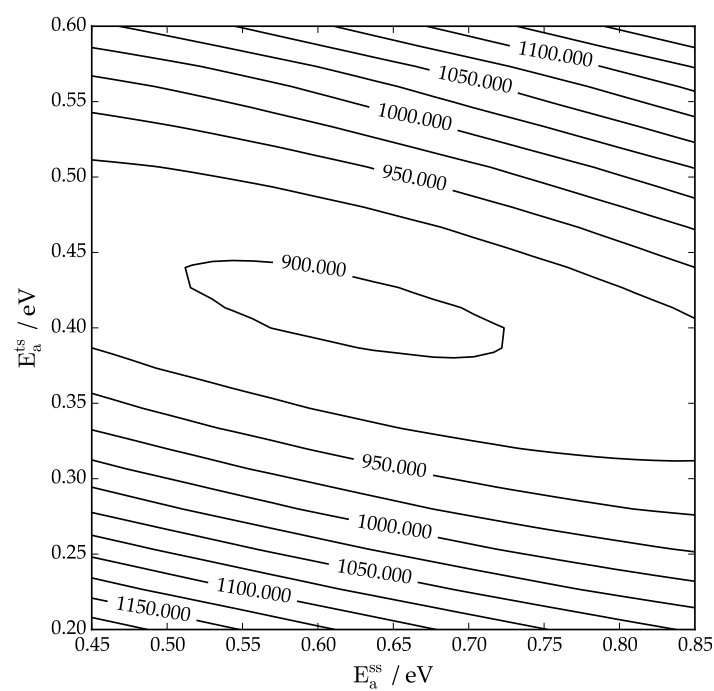

(b) Residual vs activation energy (ts- and ssreaction)

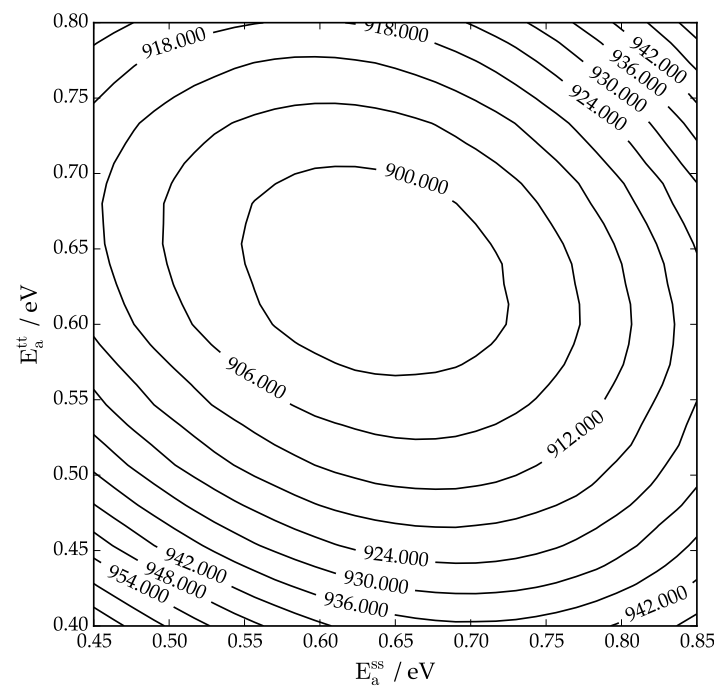

(c) Residual vs activation energy (tt- and ssreaction)
Figure 7.14: Sensitivity of the fit residual vs activation energies on $\operatorname{Pt}(111)$ : All panels show a contour plot of the total fit residual as a function of two activation energies.

The upper panel shows the contour plot for the $\mathrm{tt}-$ and the ts-reaction, the middle panel the ts- and the ss-reaction and the lower panel the $\mathrm{tt}$ - and the ssreaction.

The gradient along $E_{a}^{t s}$ in the upper and the middle panel is stronger than the gradients along $E_{a}^{t t}$ and $E_{a}^{s s}$, which results in an oval shape of the contour lines.

The combination of $\mathrm{E}_{a}^{\mathrm{tt}}$ and $E_{a}^{s s}$ in the lower panel yields more or less round contour lines, which indicates equal sensitivity of the residual on these two parameters.

Cross-correlations between parameters are present if the gradient along one diagonal is stronger than along the other diagonal. This behavior can be seen in the middle panel, where the contour lines are slanted. Reproduced with permission from Ref. [46]. 
The local optimization varied $\left[\mathrm{O}_{t}\right]$ and $\left[\mathrm{O}_{s}\right]$ per kinetic trace, see Python code structure on $p$. 117 .

The Langmuir fit to the titrations is shown in figure 7.4 on $p .107$.

\subsubsection{Steady-state O-atom concentrations}

Since the O-atom coverages on terrace and step sites were allowed to vary in the local optimization, we now compare the optimization result to the input values. The most important comparison is the resulting total $\mathrm{O}$-atom coverage $\left[\mathrm{O}_{\mathrm{a}}\right]$ against the experimental titration values, which is shown in the upper panel in figure 7.15. The experimentally determined values (grey dots with error bars) were previously fitted by the Langmuir-like function in red. The total O-atom concentration determined in the local optimization, $\left[\mathrm{O}_{\mathrm{a}}\right]=\left[\mathrm{O}_{\mathrm{t}}\right]+\left[\mathrm{O}_{\mathrm{s}}\right]$, is plotted as blue squares in the upper panel. The fit result agrees well with the experimentally determined values.
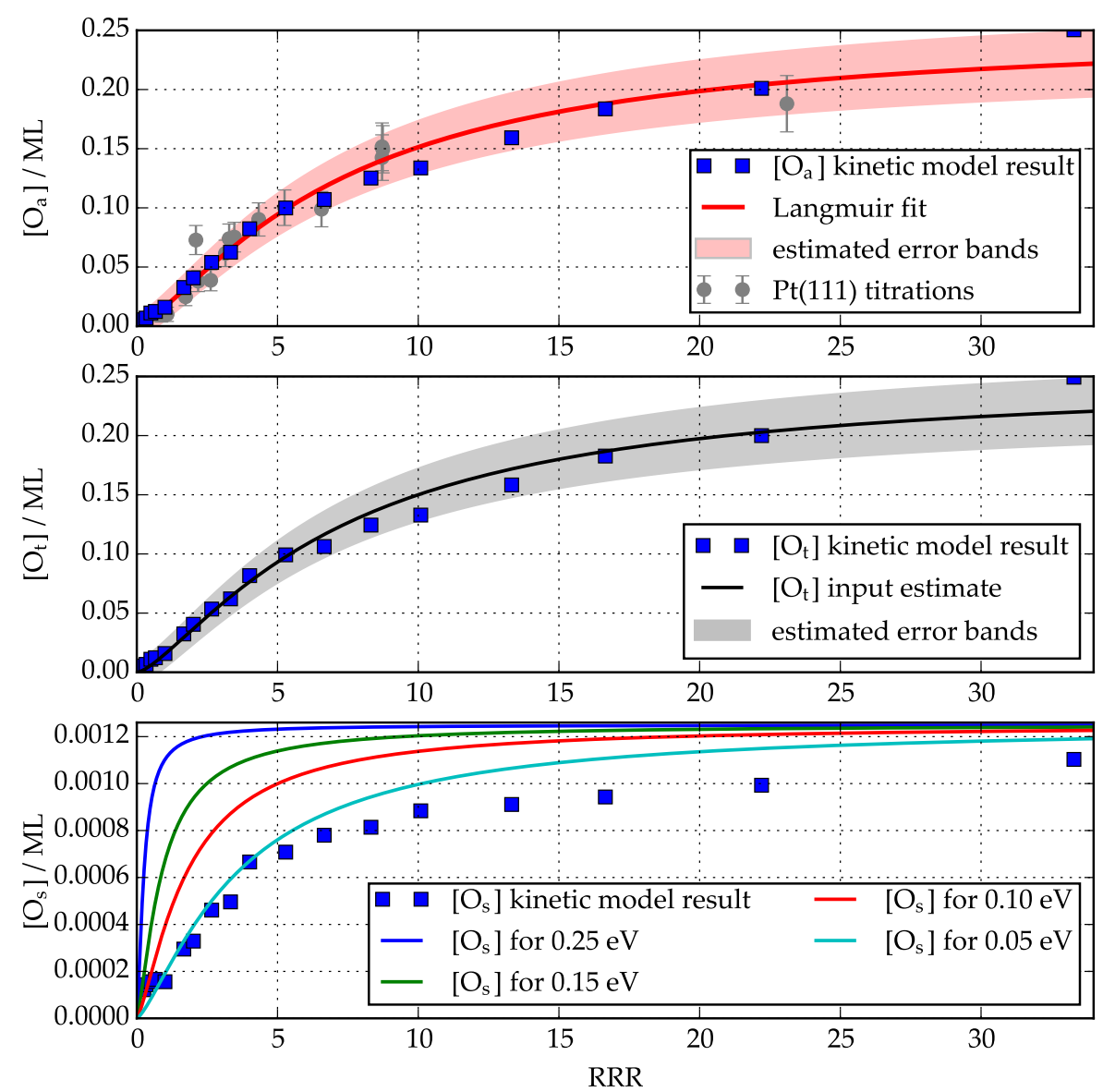

Figure 7.15: Comparison of the kinetic model result O-atom concentrations to input values on $\mathrm{Pt}(111)$ at $613 \mathrm{~K}$ : The upper panel compares the total O-atom concentration, the middle panel the concentration on terraces and the bottom panel the concentration on steps. The curves in the bottom panel are the result of the partition function calculation using the binding energy differences given in the legend.

The middle panel in figure 7.15 compares $\left[\mathrm{O}_{\mathrm{t}}\right]$ to the input estimate. The input values were calculated by dividing $\left[\mathrm{O}_{a}\right]$ from the Langmuir fit into $\left[\mathrm{O}_{t}\right]$ and $\left[\mathrm{O}_{s}\right]$ according to the equilibrium distri- 
bution based on the partition function, see section 7.3.2 on p. 112. The error bands of the Langmuir fit were used as boundaries for $\left[\mathrm{O}_{t}\right]$ in the local optimization. The kinetic model is able to reproduce the experimentally determined behavior of $\left[\mathrm{O}_{t}\right]$ vs RRR quite accurately. Furthermore, the kinetic model result does not systematically push against the boundaries. This suggests that the good agreement is not just a result of the boundaries but that the kinetic model would deliver a similar result even without boundaries.

Lastly, we compare $\left[\mathrm{O}_{s}\right]$ against several estimates based on different equilibrium distributions in the lower panel. The partition function was used to calculate the equilibrium distribution for four different terrace-step binding energy differences, see section 7.3 on p. 108 . We find that the kinetic model $\left[\mathrm{O}_{s}\right]$ is lower than expected. If we compare the kinetic model values (blue squares) to the blue line, which is the prediction using a binding energy difference of $0.25 \mathrm{eV}$, the discrepancy is drastic. Under the assumption that the kinetic model result is correct, two explanations come to mind. First, the reported binding energy difference of $0.26 \mathrm{eV}$ by Morimoto et al. [59, 60] might be overestimated. An energy difference of $0.05 \mathrm{eV}$, which is close to the kinetic model result, would be a better fit. However, the densityfunctional theory (DFT) result by Morimoto et al. is expected to be quite accurate for energy differences. Second, the assumption that $\left[\mathrm{O}_{s}\right]$ and $\left[\mathrm{O}_{t}\right]$ are in equilibrium might be wrong. The high reaction rates at steps preferably deplete the O-atoms on steps. Between the $\mathrm{CO}$ pulses the $\mathrm{O}$-atoms on steps can be replenished by the $\mathrm{O}_{2}$ beam or by diffusion from $\mathrm{O}$-atoms on terraces to steps. If these two processes are not sufficient to establish thermodynamic equilibrium, the system will establish different steady-state concentrations.

An analogous comparison is presented in figure 7.16 for the kinetic model result on $\mathrm{Pt}(332)$ at $593 \mathrm{~K}$. A different temperature than the one fot $\operatorname{Pt}(111)$ is used because the titration was carried out at this temperature. The upper panel shows total $\mathrm{O}$-atom concentration $\left[\mathrm{O}_{\mathrm{a}}\right]$ as determined in the titration experiment (grey circles and triangles) and the related Langmuir fit (red curve). The kinetic model results for $\left[\mathrm{O}_{\mathrm{a}}\right]$ (blue squares) match this prediction nicely.

The middle panel shows the comparison between input and result for $\left[\mathrm{O}_{t}\right]$. The error bands were used as boundaries for the fit, so the agreement is not surprising. Within the boundaries, the result values are close to the $\left[\mathrm{O}_{t}\right]$ prediction (black curve) except at high RRRs. At RRR values of 10, 13.3 the result is close to the boundary and at 16.6 $\left[\mathrm{O}_{t}\right]$ is equal to the limiting value. If we examine the related kinetic trace (bottom right trace in figure A.2d on p. 192), no obvious mismatch can be found. One could thus speculate that without boundaries the $\left[\mathrm{O}_{\mathrm{t}}\right]$ value at an RRR of 16.6 would only change slightly.

The comparison of $\left[\mathrm{O}_{s}\right]$ to thermodynamic equilibrium calculations shows that the kinetic model result values are best represented
In this case steadystate refers to the concentrations being similar for each $\mathrm{CO}$ pulse. 

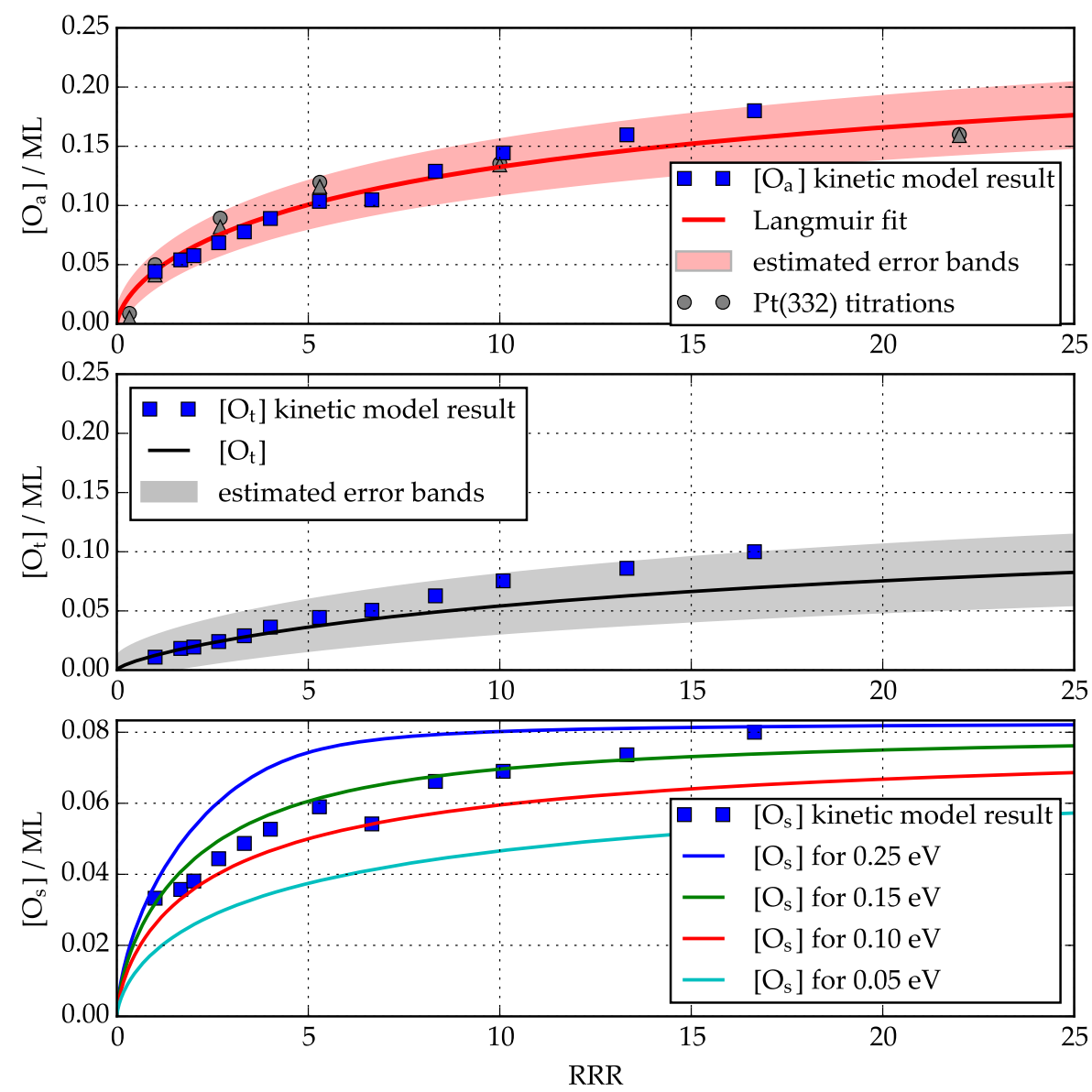

Figure 7.16: Comparison of the kinetic model result O-atom concentrations to input values on $\mathrm{Pt}(332)$ at $593 \mathrm{~K}$ : The upper panel compares the total O-atom concentration, the middle panel the concentration on terraces and the bottom panel the concentration on steps. The curves in the bottom panel are the result of the partition function calculation using the binding energy differences given in the legend.

by a binding energy difference of $0.15 \mathrm{eV}$. This is much closer to the literature value by Morimoto et al.. Again, it is not clear whether this suggests a different binding energy difference at thermodynamic equilibrium or simply that system is not at thermodynamic equilibrium. 
[38] C.T. Campbell, G. Ertl, H. Kuipers, and J. Segner. "A molecular beam investigation of the interactions of $\mathrm{CO}$ with a $\mathrm{Pt}(111)$ surface." In: Surface Science Letters 107.1 (May 1981), A222. DoI: 10. 1016/0167-2584 (81) 90554 - 5 (cit. on pp. 46, 62, 100).

[46] Jannis Neugebohren, Dmitriy Borodin, Hinrich W. Hahn, Jan Altschäffel, Alexander Kandratsenka, Daniel J. Auerbach, Charles T. Campbell, Dirk Schwarzer, Dan J. Harding, Alec M. Wodtke, and Theofanis N. Kitsopoulos. "Velocity-resolved kinetics of site-specific carbon monoxide oxidation on platinum surfaces." In: Nature 558.7709 (June 2018), pp. 280-283. DOI: $10.1038 /$ s41586-018-0188-x (cit. on pp. 79-82, 123, 130, 132, 134, 146, 147).

[49] J. Segner, C. T. Campbell, G. Doyen, and G. Ertl. “Catalytic oxidation of $\mathrm{CO}$ on $\operatorname{Pt}(111)$ : The influence of surface defects and composition on the reaction dynamics." In: Surface Science 138.2-3 (Mar. 1984), pp. 505-523. DOI: 10.1016/0039-6028 (84) 90262-0 (cit. on pp. 81, 88, 96, 129, 135-137).

[59] Ryosuke Jinnouchi, Kensaku Kodama, and Yu Morimoto. “DFT calculations on $\mathrm{H}, \mathrm{OH}$ and $\mathrm{O}$ adsorbate formations on $\mathrm{Pt}(111)$ and $\mathrm{Pt}(332)$ electrodes." In: Journal of Electroanalytical Chemistry 716 (Mar. 2014), pp. 31-44. DoI: 10.1016/j . j elechem. 2013. 09. 031 (cit. on pp. 90, 112, 125, 143).

[6o] A. Nagoya, Ryosuke Jinnouchi, Kensaku Kodama, and Yu Morimoto. "DFT calculations on $\mathrm{H}, \mathrm{OH}$ and $\mathrm{O}$ adsorbate formations on $\mathrm{Pt}(322)$ electrode." In: Journal of Electroanalytical Chemistry 757 (Nov. 2015), pp. 116-127. DOI: 10.1016/j . jelechem . 2015.09.009 (cit. on pp. 90, 112, 125, 137, 143).

[61] C. T. Campbell, G. Ertl, H. Kuipers, and J. Segner. "A molecular beam study of the adsorption and desorption of oxygen from a Pt(111) surface." In: Surface Science 107.1 (May 1981), pp. 220-236. DOI: 10 . 1016/0039-6028(81 ) 90622 - 1 (cit. on p. 97).

[62] R. Lewis and R. Gomer. "Adsorption of oxygen on platinum." In: Surface Science 12.2 (Oct. 1968), pp. 157-176. DoI: 10.1016/ 0039-6028 (68) 90121 - 0 (cit. on p. 97).

[63] J. D. Lambert. Numerical Methods for Ordinary Differential Systems: The Initial Value Problem. New York: Wiley, 1991 (cit. on p. 99).

[64] Adam C. Hindmarsh. “ODEPACK, A Systematized Collection of ODE Solvers." In: IMACS Transactions on Scientific Computation. Ed. by R. S. Stepleman. Vol. 1. Amsterdam: North-Holland, 1983, pp. 55-64 (cit. on p. 99). 
[65] G. Ertl, M. Neumann, and K. M. Streit. "Chemisorption of CO on the Pt(111) surface." In: Surface Science 64.2 (1977), pp. 393410. DOI: 10. 1016/0039-6028 (77) 90052 - 8 (cit. on p. 100).

[66] H. Steininger, S. Lehwald, and H. Ibach. "On the adsorption of CO on Pt(111)." In: Surface Science 123.2-3 (1982), pp. 264282. DOI: 10. 1016/0039-6028 (82) 90328- 4 (cit. on p. 100).

[67] John L. Gland and Edward B. Kollin. "Vibrational characterization of carbon monoxide oxidation on the Pt(111) surface." In: Surface Science 151.1 (1985), pp. 260-270. DOI: 10 . 1016/0039 6028 (85) 90465- 0 (cit. on pp. 100, 136).

[68] J. Liu, M. Xu, and F. Zaera. "Determination of the rate limiting step in the oxidation of $\mathrm{CO}$ on $\mathrm{Pt}(111)$ surfaces." In: Catalysis Letters 37.1-2 (1996), pp. 9-13. DOI: 10 . 1007 / BF00813512 (cit. on pp. 100, 143).

[69] H. P. Bonzel and R. Ku. "On the kinetics of oxygen adsorption on a Pt(111) surface." In: Surface Science 40.1 (Oct. 1973), pp. 85101. DOI: 10. 1016/0039-6028 (73) 90053-8 (cit. on p. 100).

[70] Cansin Badan, Rachael G. Farber, Yasmine Heyrich, Marc T. M. Koper, Daniel R. Killelea, and Ludo B. F. Juurlink. "Step-Type Selective Oxidation of Platinum Surfaces." In: The Journal of Physical Chemistry C 120.40 (Oct. 2016), pp. 22927-22935. DOI: 10. 1021/acs. jpcc. 6 b05482 (cit. on p. 101).

[71] Bene Poelsema, Laurens K. Verheij, and George Comsa. "HeScattering Investigation of CO Migration on Pt(111)." In: Physical Review Letters 49.23 (Dec. 1982), pp. 1731-1735. DOI: 10 . 1103/PhysRevLett. 49.1731 (cit. on pp. 114, 139, 140). 


\subsection{DisCUSSION OF THE RESUlts}

During the investigation of $\mathrm{CO}$ oxidation on atomically flat and stepped platinum surfaces we obtained a broad range of experimental information. Information on the dynamics of the reaction, such as product angular and velocity distributions, was found to agree with literature. Other results, especially the reaction mechanism, are very difficult to compare due to the various different hypotheses reported in literature. In order to facilitate the respective discussion, the various experimental findings are arranged in separate subsections. At the beginning of each subsection I give a brief review of selected milestone papers.

\subsubsection{Angular distribution}

The dynamics of the reaction were first investigated by Palmer and Smith [54] on an epitaxially grown $\mathrm{Pt}$ (111) surface at elevated temperatures above $500 \mathrm{~K}$. They found the angular distribution of the $\mathrm{CO}_{2}$ desorption to be sharply peaked about the surface normal exhibiting a $\cos ^{\mathrm{d}}(\vartheta)$ distribution with $4 \leqslant \mathrm{~d} \leqslant 6$. The sharpness was found to increase for smoother surfaces. Later investigations by the same authors found the angular distribution to be composed of a higher order cosine $\left(\cos ^{6}(\vartheta)\right)$ distribution superimposed on a broader component [72, 73]. The bimodal angular distribution of the $\mathrm{CO}_{2}$ products was later confirmed as $\alpha \cos (\vartheta)+(1-\alpha) \cos ^{7-8}(\vartheta)$ in various reports [47-50].

A typical angle-integrated ion image and the extracted angular distribution recorded at Beamer II is shown in figure 8.1. Measuring and extracting the angular distribution with high accuracy was quite challenging for several reasons:

First, only about $30^{\circ}$ solid angle $(\vartheta)$ could be measured, with space charge from the trace of the incoming beam limiting one side and the ion optics limiting the other side. The small experimentally accessible range of angles becomes even more critical when taking the extent of the surface into account. Instead of a point source the reactive region on the surface is about the diameter/overlap of the incoming beams, i.e. $3 \mathrm{~mm}$. For the narrow hyperthermal channel this translates into about 75 pixel source size compared to a scattered signal about 350 pixel broad. A proper fit to the angular distribution would

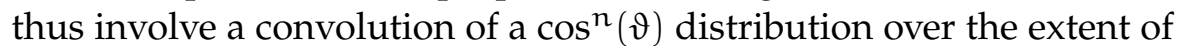
the source, e.g. a gaussian distribution with $3 \mathrm{~mm}$ FWHM. The extent

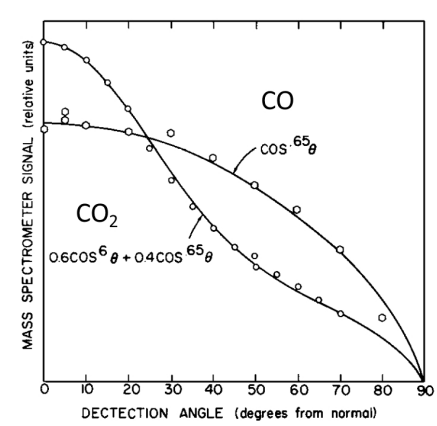

Palmer [72]

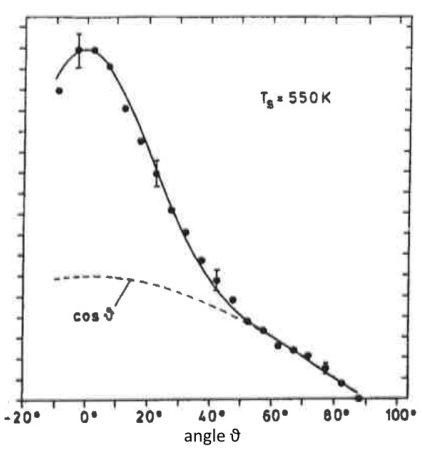

Poehlmann et al. [50] 

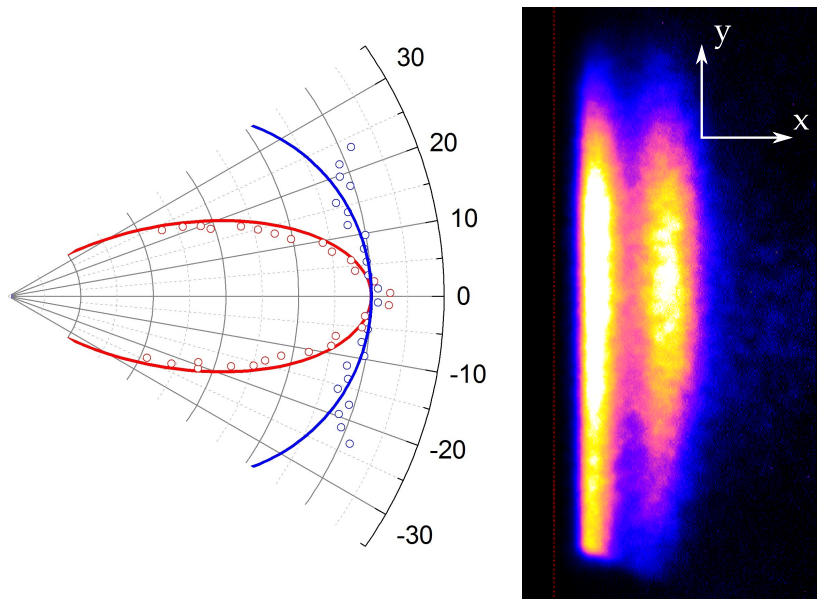

Figure 8.1: Angular distribution and the corresponding ion image on the right. The origin of the angular distribution coincides with the surface coordinates. The thermal $\mathrm{CO}_{2}$ (blue circles) is compared to a $\cos (\vartheta)$ distribution (blue curve) and the hyperthermal component (red circles) is compared to a $\cos ^{8}(\vartheta)$ distribution (red curve). Reproduced with permission from Ref. [46].

of the source can be neglected when employing VMI techniques, as shown in section 4.4. This would allow the resolution to be improved significantly.

Second, the normalization of the signal was quite difficult. There are three major factors biasing the ion signal intensity:

1. Misalignment of the grids can change the height of the laser between repeller and extractor grid when scanning the focus along $\mathrm{y}$. This causes a bias along y.

2. The grids might not be flat. This biases in the xy plane.

3. The sensitivity of the MCP is not uniform. This also biases in the xy plane.

The laser intensity during the angular scan was constant, but the laser focus height between the repeller and the extractor grid varies slightly when scanning the focus lens. This acts on the TOF and changes the signal strength due to the $\mathrm{MCP}$ gate. Additionally, non-flatness of the extractor grid could cause bunching of the signal into certain areas. And on top of that, the MCP sensitivity is not uniform across the whole screen. Especially the heavily used region in the middle of the scattering region is less sensitive.

Most of these issues can be corrected for by measuring the signal intensity of a uniformly distributed background gas in the chamber, ideally of the same mass to keep the same TOF. The velocities of the background gas are lower than those of the $\mathrm{CO}_{2}$ products. While the background gas signal on the detector partially overlaps with the thermal $\mathrm{CO}_{2}$ channel, there is no overlap with the hyperthermal channel. 
In order to measure the MCP sensitivity where the fast $\mathrm{CO}_{2}$ product is detected, we have to move the laser focus along x. As a consequence the ions pass through a different part of the extractor grid, which might cause a different bunching. We thus used the intensity distribution of the background gas along $y$ to correct both the thermal and the hyperthermal angular distribution. Effects such as inhomogeneous MCP sensitivity could not be corrected for the hyperthermal channel.

The ion image shown on the right side of figure 8.1 is velocityto-flux transformed and background corrected. The angular distributions on the left side of figure 8.1 were extracted from this ion image and for both channels the intensity along $y$ was corrected using an ion image of uniformly distributed $\mathrm{CO}_{2}$ background gas (not shown).

Given the difficult analysis of the angular distribution measurement, we only compare the thermal channel to a $\cos (\vartheta)$ distribution and the hyperthermal channel to $a \cos ^{8}(\vartheta)$ distribution and find good qualitative agreement.

\subsubsection{Speed and kinetic energy distribution}

The velocity distribution of $\mathrm{CO}_{2}$ was first measured by Auerbach et al. [53]. In a molecular beam experiment on $\mathrm{Pt}$ (poly) the temperature characterizing the kinetic energy distribution was measured to be far above the surface temperature of $880 \mathrm{~K}$. While Auerbach et al. found the velocities to decrease at higher angles, the bimodality in speed was first reported by Poehlmann et al. [47] in a molecular beam experiment at temperatures $500-800 \mathrm{~K}$. Allers et al. [48] also found a bimodality in the kinetic energy distribution in a temperature programmed reaction spectroscopy (TPRS) experiment.

The velocity distribution, as shown in fig. 8.2, reveals two components - a slow component fitted by a sub-thermal MB speed distribution at a temperature of $483 \mathrm{~K}$ compared to a surface temperature of $613 \mathrm{~K}\left(\mathrm{~T}_{\mathrm{MB}}=0.77 \mathrm{~T}_{\mathrm{S}}\right)$, and a hyperthermal component showing a broader width $(894 \mathrm{~K})$ and a velocity shift of $910 \mathrm{~m} \mathrm{~s}^{-1}(185 \mathrm{meV})$.

A sub-thermal velocity distribution can be explained following the principles of detailed balance as reported by Comsa and David [37]. In my own words: The speed distribution of molecules desorbing from a surface in thermal and mass equilibrium with the surrounding gas must always be equal to the speed distribution of adsorbing molecules, otherwise the surface would heat up or cool down. A different distribution of desorbing molecules is also forbidden by the second law of thermodynamics because it could otherwise be exploited in a molecular machine.

The sticking probability of a non-activated physisorption process decreases with incident velocity, which surely applies to the weakly bound $\mathrm{CO}_{2}$ [74]. The distribution of adsorbing and desorbing mole-

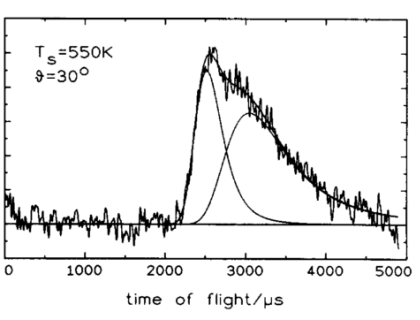

Poehlmann et al. [47] 
The mean kinetic energy was introduced in section 4.2.4: Speed distributions in surface scattering experiments.

dwell time: time on the surface prior to reaction

I did not check whether the error from this assumption has the right magnitude to account for the discrepancy.

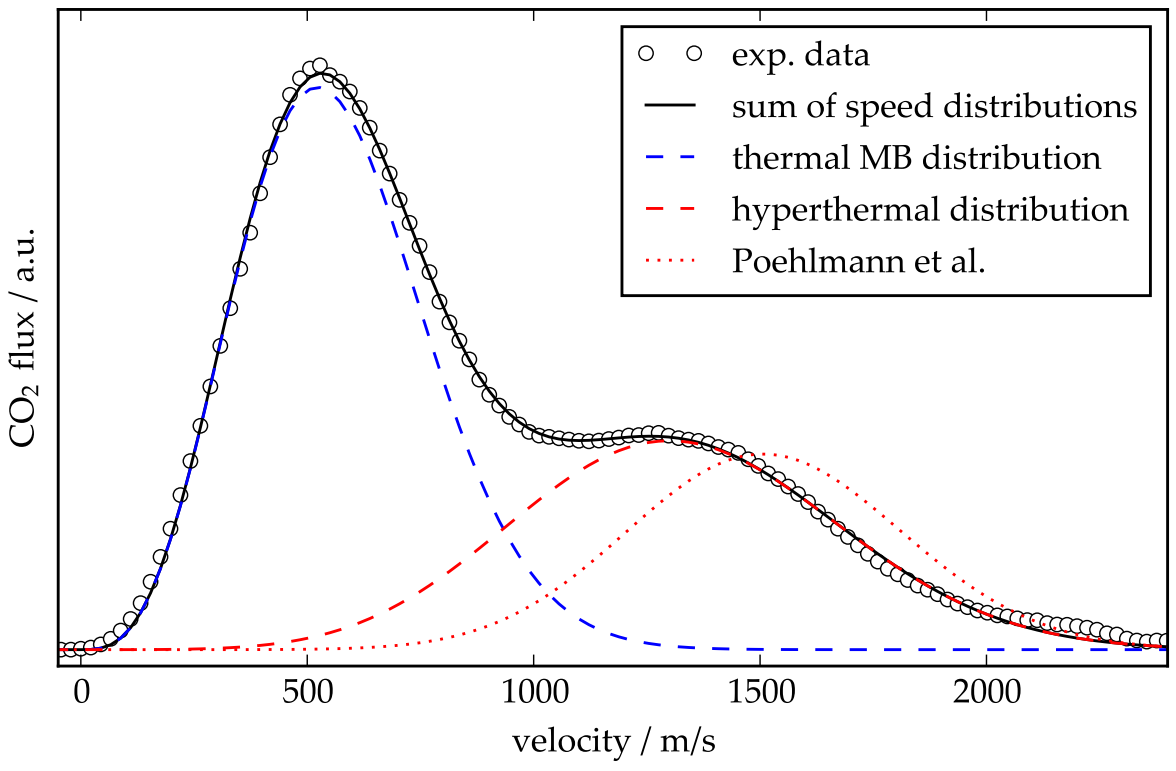

Figure 8.2: Comparison of the flux velocity distribution at $613 \mathrm{~K}$, as shown in section 6.3, to the velocity distribution reported by Poehlmann et al.. The slight mismatch is explained in the text. Adapted with permission from Ref. [46].

cules is thus shifted to smaller velocities and leads to a sub-thermal speed distribution. A molecule with a significant adsorption barrier on the other hand would be expected to show a hyperthermal velocity distribution.

The mean kinetic energy of the thermal product $\mathrm{CO}_{2}$ flux along the surface normal can be described as

$$
\left\langle\mathrm{E}_{\mathrm{kin}}\right\rangle_{\perp}=\frac{1}{2} \mathrm{~m}\left\langle v^{2}\right\rangle=2 \mathrm{k}_{\mathrm{B}} \mathrm{T}_{\mathrm{S}},
$$

with the surface temperature $T_{S}$. Note that this distribution assumes that the product is in thermal equilibrium with the surface. If the $\mathrm{CO}_{2}$ molecules have a velocity shift $\mathrm{v}_{0}$, the analytical expression for the mean kinetic energy becomes quite complicated. I prefered to solve the integral numerically. Table 8.1 below compares our experimentally determined flux velocity distribution for the hyperthermal channel to literature values. When comparing our hyperthermal distribution in figure 8.2 to the distribution by Poehlmann et al. ( $550 \mathrm{~K}$ $+385 \mathrm{meV}$, dotted red line) the literature velocities are significantly higher. This also becomes clear when comparing the mean kinetic energy shown in table 8.1. The literature values were extracted from TOF distributions assuming that the hyperthermal channel shares the same dwell time on the surface prior to reaction as the thermal channel. For shorter dwell times the kinetic energy would decrease, which would then support our results. An overestimation of the dwell time is likely considering that the hyperthermal channel showed shorter 
Table 8.1: Kinetic energy distributions. The temperature $T$ defines the width of the distribution and $E_{b}$ is the energy offset/shift. $\left\langle\mathrm{E}_{\mathrm{kin}}^{\mathrm{HT}}\right\rangle$ is the mean kinetic energy.

\begin{tabular}{lcc}
\hline & $\begin{array}{c}\text { HT distribution } \\
\mathrm{T}+\mathrm{E}_{\mathrm{b}}\end{array}$ & $\left\langle\mathrm{E}_{\mathrm{kin}}^{\mathrm{HT}}\right\rangle$ \\
\hline This work, at 613 K & $894 \mathrm{~K}+190 \mathrm{meV}$ & $430 \mathrm{meV}$ \\
Allers et al. [48] & & $520 \mathrm{meV}^{\mathrm{a}}$ \\
Poehlmann et al. [50] & $550 \mathrm{~K}+385 \mathrm{meV}^{\mathrm{b}}$ & $490 \mathrm{meV}^{\mathrm{a}}$ \\
\hline
\end{tabular}

a: The mean kinetic energy from both reports was interpolated to match our temperature of $613 \mathrm{~K} .{ }^{\mathrm{b}}$ : The kinetic energy distribution was measured at $550 \mathrm{~K}$. Poehlmann et al. assumed the width to be thermal and thus obtained the high value for $E_{b}$.

dwell times than the thermal channel at all conditions investigated in this work.

While a comparison of mean kinetic energy is straightforward, the actual resulting flux velocity distribution is not just shifted but also broadened relative to the distribution at surface temperature. The exoergicity of the reaction is distributed over several degrees of freedom, such as translation, rotation and vibration, and partially transferred to the surface. The amount of energy transferred to translation $E_{b}$ will be a distribution, not a quantized value. The spread of the distribution will be added to the inherent spread of the MB distribution. The width of the resulting $\left(T+\left\langle E_{b}\right\rangle\right)$ distribution cannot be described by the surface temperature anymore.

As stated above, the exothermicity of the reaction is likely distributed over several degrees of freedom and partially transfered to the surface. In order to assess the energy spread we plot the data shown in figure 8.2 as a function of kinetic energy. The transformation from velocity to energy is based on

$$
\begin{aligned}
f(v) d v & =g(E) d E \\
\Leftrightarrow f(v) \frac{d v}{d E} & =g(E)
\end{aligned}
$$

with $d v / d E=1 / m v$. We convert the experimental data by replacing the velocity with the kinetic energy and dividing the related $y$-value by $m v$.

Two features of the distribution become much clearer when plotting as a function of kinetic energy: The hyperthermal channel has a higher kinetic energy than the thermal channel and the width of the distribution is much broader. The maximum kinetic energy observed is about $1000 \mathrm{meV}$. A thermal $\mathrm{CO}_{2}$ product would have a mean kinetic energy of $2 k_{B} T_{M B}$, which is $83 \mathrm{meV}$ at $T_{M B}=0.77 \times T_{S}=483 \mathrm{~K}$. 
The kinetic energy release from the reaction at terraces has been estimated by a fellow PhD student, Jan Altschäffel, using AIMD trajectories. The trajectories started on the product side of the transition state as determined in a minimum energy path (MEP) calculation using DFT-GGA and the RPBE functional. The transition state geometry for reaction of carbon monoxide with oxygen on $\mathrm{Pt}(111)$ terraces is shown in figure 8.3. The kinetic energy release of $660-750 \mathrm{meV}$, indi-

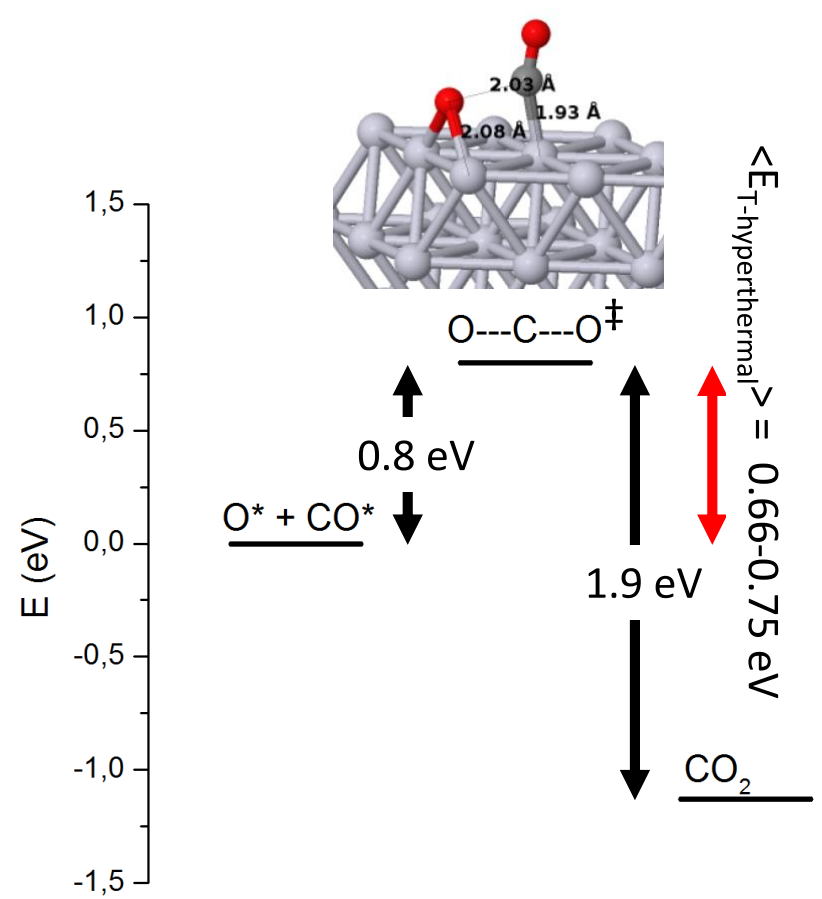

Figure 8.3: Minimum energy path calculation by Jan Altschäffel. Reproduced with permission from Ref. [46].

cated by the red arrow, was obtained for a flexible slab at $\mathrm{T}=\mathrm{o} \mathrm{K}$. The predicted kinetic energies are slightly higher than the ones measured in this work. AIMD trajectories for a slab at a higher temperature are expected to lead to a larger spread of kinetic energies but no significant shift. Including energy transfer to other $\mathrm{CO}$ molecules or $\mathrm{O}$ atoms adsorbed near the reactants might lead to lower kinetic energies. The exoergicity of the reaction was calculated to be $1900 \mathrm{meV}$. 


\subsubsection{CO oxidation mechanism on platinum}

The oxidation of $\mathrm{CO}$ on platinum surfaces has been a subject of investigations for over two centuries, starting with the invention of the explosion proof lamp. The design presented in 1816 by Humphrey Davy [75] enclosed a flame with a fine platinum gauze, as shown in the sketch on the side. In 1825 W. Henry [76] observed CO "to be capable of uniting with oxygen at the temperature of the atmosphere, by means of the (platinum) sponge".

As part of his effort to characterize catalytic processes on surfaces [7779] I. Langmuir reported the mechanism of the oxidation of $\mathrm{CO}$ on platinum [80] in 1922. He first found that the reaction rate was "proportional to the pressure of the oxygen, but inversely proportional to the pressure of carbon monoxide" at low temperatures and concluded that the $\mathrm{CO}$ molecules form an oriented, non-reactive monolayer on the surface. As the $\mathrm{CO}$ desorption rate increases with higher temperatures, the catalyst poisoning can be circumvented by either increasing the temperature or decreasing the partial pressure of CO. Information on the reaction mechanism can then be obtained by comparing the reaction rate to the impingement rate of the reactants on the heated catalytic filament.

With the advent of modern experimental tools such as ultra-high vacuum technology, single crystal studies and molecular beams [81] in the 1960s the investigation of chemical kinetics and dynamics at surfaces became a hot topic in physical chemistry. Especially the oxidation of $\mathrm{CO}$ on platinum surfaces received a great deal of attention. A detailed summary of the early investigations can be found in the 1979 review by Engel and Ertl [82]. After an initially controversial discussion $[83,84]$ the community soon concluded that the reaction followed a Langmuir-Hinshelwood type reaction mechanism [53-55].

In 1980 Campbell, Ertl and coworkers [55] used a molecular beam experiment to study $\mathrm{CO}$ oxidation on a $\mathrm{Pt}(111)$ crystal. Assuming a simple LH reaction mechanism they reported the activation energy $\mathrm{E}_{\mathrm{LH}}$ of the reaction to decrease from $24.1 \mathrm{kcal} / \mathrm{mol}$ to $11.7 \mathrm{kcal} / \mathrm{mol}$ with increasing oxygen coverage. They attributed the change in activation energy to "the corresponding increase in the energy of the adsorbed reactants".

In their 1984 follow-up publication Segner et al. [49] reported several interesting findings. First, they reported that both channels, i.e. processes with two different activation energies, operate simultaneously. Second, they concluded "that the transition state remains essentially the same (even if $E_{L H}$ is changing), but that a certain fraction $\alpha$ of the molecules formed is intermediately trapped in the $\mathrm{CO}_{2, a d}$ potential well, while the other fraction leaves the surface directly".

In a TPD experiment reported by Gland and Kollin in 1983 the activation energy was found to change with coverage at low temper-
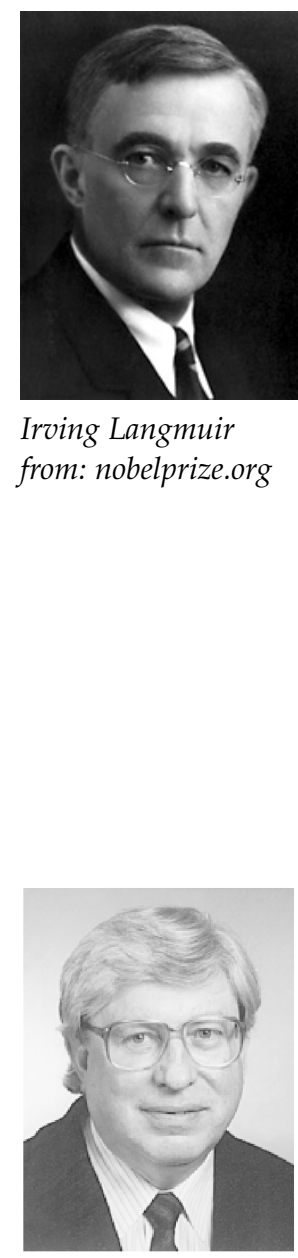

Irving Langmuir from: nobelprize.org 
atures $(330 \mathrm{~K})$, but the authors concluded that a single reaction mechanism dominates [86]. The authors further defined the reaction mechanism as "reaction of mobile CO along oxygen island perimeters" [67].

In an isotope mixing experiment in 1986 Akhter et al. [87] tried to verify this hypothesis but instead found homogeneous reactivity across the oxygen islands. The authors pointed out that "a model in which diffusion into and out of islands adequately accounts for those observations" is conceivable.

In 1992 Yates et al. found that "the elementary step producing $\mathrm{CO}_{2} \ldots$ is structure sensitive". By adsorbing different isotopic CO molecules on steps and terraces, the terrace $\mathrm{CO}$ was found to react exclusively at temperatures below $200 \mathrm{~K}$. Exchange between step and terrace $\mathrm{CO}$ above this temperature prevented investigation of the known TPRS peaks at higher temperature.

The reaction-at-perimeters hypothesis was further supported by scanning tunneling microscope (STM) experiments by Wintterlin et al. [88] in 1997 revealing a reaction rate proportional to the perimeter length of oxygen islands at low temperatures. The authors concluded that the assumption of an immobile oxygen layer at $250 \mathrm{~K}$ by Akhter $e t$ al. was flawed.

Until now the accepted reaction mechanism on platinum at elevated temperatures included only one reaction pathway with a single elementary step $[48,49,55]$. The different activation energies observed were attributed "to an increase in energy of the adsorbed reactants" with oxygen coverage [55] or to a "change in average interadsorbate distance" [48]. Models with independent reaction channels or different reaction sites have been suggested, but not verified experimentally.

The measurement of velocity-resolved kinetic traces is to my knowledge the first report showing the different lifetimes of the thermal and hyperthermal $\mathrm{CO}_{2}$ on the surface. It allows us to reject any proposed mechanism based on a single reaction channel because this would lead to similar lifetimes of the two channels. I want to illustrate this important point by discussing a few possible interpretations of the experimental findings:

"The different lifetimes of the two channels could be due to a change in the branching ratio after the transition state during the $\mathrm{CO}$ pulse."

One could raise the argument that the branching into thermal and hyperthermal channel after the transition state is influenced by the chemical environment, i.e. interaction between adsorbates. The adsorbate concentration could change significantly during the reaction following a single pulse of $\mathrm{CO}$. $\mathrm{CO}$ molecules that react early would experience strong interactions at the transition state, while $\mathrm{CO}$ molecules that react late would experience weaker interactions.

Since the CO beam flux is very small, a large change in the chemical environment by consumption of a large quantity of oxygen can 
be ruled out. The flux is also small enough to exclude interactions between $\mathrm{CO}$ molecules on the surface.

One could also argue that the interactions controling the branching ratio depend on the position of the reacting oxygen atom, e.g. on a terrace or next to step or defect sites. However, if the reaction channel up to the transition state is similar, all oxygen atoms would be consumed equally fast and the lifetimes would be similar.

This raises the question whether the experimental findings can be explained by a model involving just two different kinds of oxygen atoms:

"The different lifetimes could be due to two different oxygen species of which one is quickly depleted during a single pulse of CO."

Reactions with one oxygen species could produce the quickly decaying part of the $\mathrm{CO}_{2}$ flux, i.e. both the hyperthermal channel and the quickly decaying component of the thermal channel. This oxygen species is consumed within one $\mathrm{CO}$ pulse, which requires the absolute number of oxygen atoms belonging to this species to be smaller than the number of $\mathrm{CO}$ molecules per pulse. The other, more abundant oxygen species would then produce the slowly decaying component of the thermal $\mathrm{CO}_{2}$. The ratio of quickly decaying $\mathrm{CO}_{2}$ flux to slowly decaying $\mathrm{CO}_{2}$ flux would be determined by the amount of $\mathrm{O}$ atoms in the first species to the amount of $\mathrm{CO}$ on the surface.

The experiment was carried out with several different $\mathrm{CO}$ beam fluxes ( $1 \%$ to $20 \% \mathrm{CO}$ in $\mathrm{He}$ and varying pulse intensities) and we found no difference in the thermal to hyperthermal ratio aside the reported change with oxygen coverage. This leads us to reject an explanation based solely on two different kinds of oxygen atoms.

The discrepancy becomes even more obvious when we specify the character of the previously undefined oxygen species as oxygen on steps and oxygen on terraces. As oxygen on terraces is the abundant species on $\mathrm{Pt}(111)$, the quickly decaying $\mathrm{CO}_{2}$ flux would have to be due to reaction with oxygen on steps. The $\mathrm{CO}$ molecular beam dose of $2 \times 10^{-5} \mathrm{ML}$ per pulse is small and most likely not large enough to consume all oxygen atoms on the $0.25 \%$ steps on $\mathrm{Pt}(111)$ - and certainly not large enough to consume all step oxygen atoms on $\mathrm{Pt}(332)$ with $16.7 \%$ steps.

As derived in the introduction in section 6.4, the only feasible explanation for the different lifetimes is two different species of $\mathrm{CO}$ on the surface. Since it is well known that both $\mathrm{CO}$ and oxygen atoms can bind to terraces and steps $[58,60,89]$, it is logical to extend the mechanism to involve these different species. The proposed mechanism has the necessary flexibility to explain a vast amount of experimental data for two surfaces with different step distributions, $\mathrm{Pt}(111)$ with $0.25 \%$ steps and $\operatorname{Pt}(332)$ with $16.7 \%$ steps.

The assignment of the thermal channel to reactions at steps also agrees with the qualitative observation by Segner et al. [49] who found 
the thermal, cosine $\mathrm{CO}_{2}$ component to increase with higher step and defect density after sputtering.

The rate coefficients obtained from the $\mathrm{Pt}(111)$ and the $\mathrm{Pt}(333)$ data are listed in table 8.2.

Table 8.2: Result: Rate coefficients on $\operatorname{Pt}(111)$ and $\operatorname{Pt}(332)$. The reaction rate coefficients are defined at an oxygen coverage of $1 \mathrm{ML}$.

\begin{tabular}{lccccc}
\hline \multirow{2}{*}{$\begin{array}{l}\text { RATE COEF- } \\
\text { FICIENTS }\end{array}$} & \multicolumn{2}{c}{$\operatorname{Pt}(111)$} & & \multicolumn{2}{c}{$\operatorname{Pt}(332)$} \\
\cline { 2 - 3 } \cline { 5 - 6 } & $E_{\mathrm{a}} / \mathrm{eV}$ & $\mathrm{A} / \mathrm{s}^{-1}$ & & $\mathrm{E}_{\mathrm{a}} / \mathrm{eV}$ & $\mathrm{A} / \mathrm{s}^{-1}$ \\
\hline$k_{\mathrm{diff}}^{\mathrm{ts}}$ & 0.3 & $2.1_{-1.8}^{+13} \times 10^{6}$ & & 0.32 & $1.5_{-1.3}^{+9.2} \times 10^{6}$ \\
$k_{\mathrm{r}}^{\mathrm{tt}}$ & $0.6 \pm 0.1$ & $3.5_{-3.0}^{+21} \times 10^{9}$ & & $0.6 \pm 0.1$ & $3.8_{-3.3}^{+23} \times 10^{9}$ \\
$k_{r}^{\mathrm{ts}}$ & $0.4 \pm 0.1$ & $5.9_{-5.1}^{+36} \times 10^{7}$ & & $0.4 \pm 0.1$ & $4.1_{-3.5}^{+25} \times 10^{7}$ \\
$k_{r}^{s s}$ & $0.65 \pm 0.1$ & $2.9_{-2.5}^{+18} \times 10^{9}$ & & $0.65 \pm 0.1$ & $2.6_{-2.2}^{+16} \times 10^{9}$ \\
\hline
\end{tabular}

The fit results for $\operatorname{Pt}(111)$ and $\operatorname{Pt}(332)$ show outstanding agreement. The largest deviation is a factor of 1.5 on the prefactor of $k_{r}^{t s}$. The consistency of the rate parameters across the two different surfaces suggests that we determined the kinetic model correctly and that we are actually reporting elementary step reaction rate constants.

\subsubsection{Effective conversion rate}

The diffusive flux $\mathrm{J}$ of adsorbates on surfaces can be described using Fick's law of diffusion,

$$
J=-D \nabla n(r, t),
$$

where $\mathrm{n}$ is the number density (number of adsorbates per unit area) at position $r$ and time $t$. From this one can derive the root-mean-squared (RMS) distance an adsorbate on a surface travels in a random walk,

$$
\sqrt{\left\langle r^{2}\right\rangle}=\sqrt{4 \mathrm{Dt}}
$$

and the root-mean-squared displacement along one dimension $\mathrm{x}$,

$$
\sqrt{\left\langle r_{x}^{2}\right\rangle}=\sqrt{2 \mathrm{Dt}}
$$

Diffusion on surfaces strongly depends on the surface temperature, due to the potential energy barrier between adsorption sites [91]. The diffusion coefficient can be expressed as

$$
D(T)=D_{0} \exp \left(-\frac{E_{\text {diff }}}{k_{B} T}\right),
$$

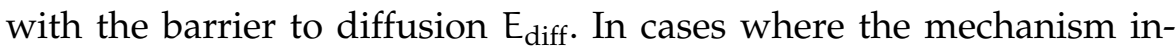
volves a site-site hopping rate $A=A_{0} \exp \left(-E_{\text {diff }} / k_{B} T\right)$, we can calculate the diffusion coefficient $D_{0}$ as

$$
\mathrm{D}_{0}=\frac{\mathrm{A}_{0} \Delta \mathrm{x}^{2}}{2 \mathrm{~d}}
$$




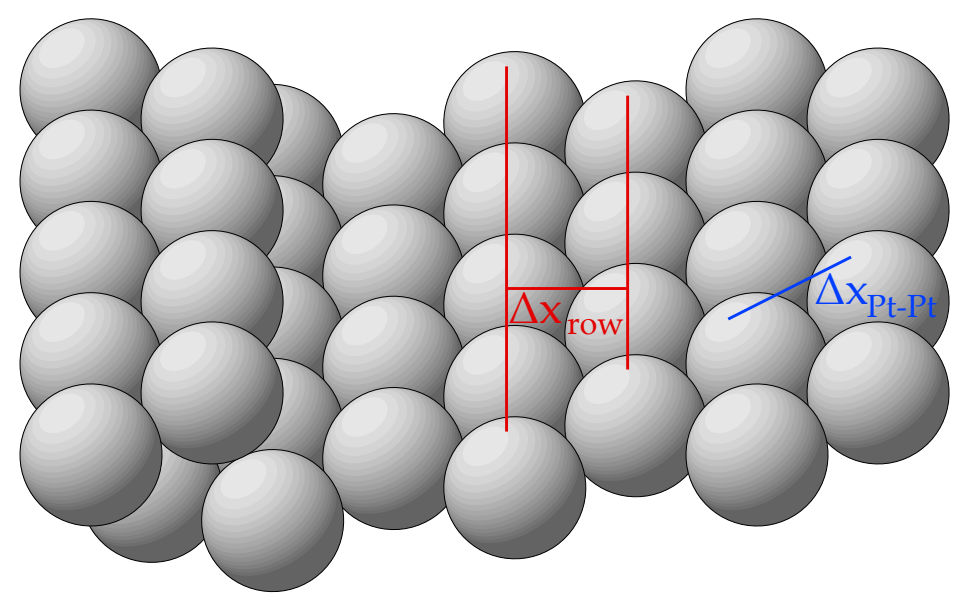

Figure 8.4: $\mathrm{Pt}(111)$ row distance vs Pt-Pt distance

where $\Delta x$ is the site-site distance and $\mathrm{d}$ is the dimensionality. i.e. $\mathrm{d}=1$ for $1 \mathrm{D}, \mathrm{d}=2$ for $2 \mathrm{D}$ diffusion. The RMS displacement can also be calculated from the hopping rate as

$$
\sqrt{\left\langle r_{x}^{2}\right\rangle}=\sqrt{\Delta x^{2} A t}
$$

For a $\mathrm{CO}$ diffusing from a terrace to a step only the movement towards the step is relevant. The distance of each hop is the distance from one row to another, as shown figure 8.4. This is also the hopping rate measured in most experiments - as the diffusion parallel to the step does not matter when measuring the CO loss to step sites, these experiments measure the $1 \mathrm{D}$ hopping rate perpendicular to steps. This factor must also be taken into account when converting the hopping rate $\mathrm{A}$ to the diffusion coefficient $\mathrm{D}$.

From a Pt-Pt distance of $2.78 \AA$ [57], indicated by the blue line in figure 8.4, we can calculate the distance between two rows as:

$$
\Delta \mathrm{x}_{\text {row }}=\Delta \mathrm{x}_{\mathrm{Pt}-\mathrm{Pt}} \times \cos \left(30^{\circ}\right)=2.4 \AA
$$

A selection of reported diffusion rate coefficients is shown in table 8.3. An investigation by Poelsema et al. [71] employed He scattering to probe the population evolution of terrace $\mathrm{CO}$ and thus measured the diffusion to steps. The diffusion coefficient $\mathrm{D}_{0}$ was calculated assuming the reported hopping rate is along one dimension. Reutt-Robey et al. measured the diffusion to steps by monitoring the increase in step site occupancy following a molecular beam pulse with time-resolved infrared spectroscopy [57]. The values reported in table 8.3 are from a follow-up publication [94], which refined the result by using a second platinum crystal with a different step density [57]. Again, the diffusion coefficient was calculated assuming a ID hopping rate. A report by Ma et al. [95] measured the diffusion of $\mathrm{CO}$ on flat and stepped $\mathrm{Pt}(111)$ surfaces using linear optical diffraction methods. A report by Wang et al. [96] studied the diffusion of CO

The selected reports include four different experimental ways to measure the diffusion. Reports such as [92] based on laser-induced thermal desorption (LITD) were omitted because of the laser-induced surface damage associated with LITD [93]. 
on $\mathrm{Pt}(111)$ terraces and steps using time-dependent tunneling current spectroscopy. The value in table 8.3 was determined for diffusion on terraces.

Table 8.3: Diffusion rate coefficients for CO on $\mathrm{Pt}(111)$.

\begin{tabular}{|c|c|c|c|c|}
\hline \multirow{2}{*}{ WORK } & \multicolumn{2}{|l|}{$E_{\text {diff }}$} & \multirow{2}{*}{$\frac{\mathrm{D}_{0}}{\mathrm{~cm}^{2} \mathrm{~s}^{-1}}$} & \multirow{2}{*}{$\frac{A_{0}}{s^{-1}}$} \\
\hline & $\mathrm{kcal} \mathrm{mol}^{-1}$ & $\mathrm{eV}$ & & \\
\hline Poelsema et al. [71] & 7.0 & 0.30 & $2.9 \times 10^{-5}$ & $10^{11}$ \\
\hline Reutt-Robey et al. [94] & 4.4 & 0.19 & $4.6 \times 10^{-7}$ & $10^{9.2}$ \\
\hline Ma et al. [95] & 4.6 & 0.20 & $6.9 \times 10^{-7}$ & \\
\hline Wang et al. [96] & 5.1 & 0.22 & $5.0 \times 10^{-7}$ & \\
\hline
\end{tabular}

Using equations 8.5-8.8 we can compare the reported diffusion rate coefficients to the effective conversion process in our kinetic model. The effective conversion rate coefficient used in the kinetic model comprises both the numerous terrace-terrace hops and the final hop from a terrace onto a step site. If we estimate the terrace length of our $0.25 \%$ step density crystal $\mathrm{Pt}(111)$ to be about 400 platinum atoms, then the mean diffusion length for randomly adsorbed $\mathrm{CO}$ molecules is about $200 \mathrm{Pt}$ atoms, i. e. $480 \AA$. From the diffusion rate coefficients in table 8.3 we calculate the RMS travel distanced $10 \mu \mathrm{s}$ after $10 \mu \mathrm{s}$ and the time required to travel a RMS distance of $480 \AA$. The results at a surface temperature of $\mathrm{T}_{\mathrm{S}}=613 \mathrm{~K}$ are shown in table 8.4.

Table 8.4: RMS diffusion displacement in $10 \mu \mathrm{s}\left(\mathrm{d}_{10 \mu \mathrm{s}}\right)$, effective conversion rate coefficient $k_{\text {conv }}$ and mean time $\tau_{\text {conv }}$ it takes to diffuse $480 \AA$ at $T_{S}=613 \mathrm{~K}$. The mean conversion time in the kinetic model for $\mathrm{CO}$ on $\mathrm{Pt}(111)$ is given as a comparison.

\begin{tabular}{|c|c|c|c|}
\hline \multirow{2}{*}{ WORK } & $d_{10 \mu s}$ & $k_{\text {conv }}$ & $\tau_{\text {conv }}$ \\
\hline & $\AA$ & $\mathrm{s}^{-1}$ & $\mu \mathrm{s}$ \\
\hline Poelsema et al. [71] & 135 & $2.0 \times 10^{4}$ & 50 \\
\hline Reutt-Robey et al. [94] & 58 & $8.6 \times 10^{3}$ & 80 \\
\hline Ma et al. [95] & 56 & $1.0 \times 10^{4}$ & 90 \\
\hline Wang et al. [96] & 39 & $7.0 \times 10^{3}$ & 120 \\
\hline Kinetic model result & & $3.9 \times 10^{3}$ & 250 \\
\hline
\end{tabular}

The effective conversion lifetime $\tau_{\text {conv }}$ used in the kinetic model is a factor 2-5 larger than the mean time necessary to diffuse a distance of 200 platinum atoms. While this is not a large factor considering the diffusion rates were extrapolated over a large temperature difference, it suggests that the final hop onto the step might be significantly 
slower than the terrace diffusion process. This would also explain the comparable effective conversion rate coefficient on $\mathrm{Pt}(332)$, which appears to be terrace-width independent.If the conversion process is limited by the terrace diffusion, the conversion rate should increase strongly on $\mathrm{Pt}(332)$, which we don't see. A different explanation for the different conversion times would be a significant in the diffusion rate due to interactions with $\mathrm{O}$-atoms. O-atoms adsorbed on steps could significantly slow down the last hop of $\mathrm{CO}$ onto steps as well.

A more accurate treatment of the conversion process would need to break the conversion into terrace-terrace hops and terrace-step hops. It would further need not only concentration gradients along the terraces, but also a distribution of different terrace sizes. A possible approach would be to simulate the diffusion and reactivity is to use a large number of compartments and calculate the reaction in each cell and the diffusion to adjacent cells numerically. A practical guide to such simulations has been formulated by Erban et al. [97].

Since the experimental data offers no further information on the conversion process, the model would have to rely on several assumptions regarding the size distribution and on literature values for the diffusion rate coefficients. Due to the complexity and as the result would have an uncertainty equal to the uncertainty of the input parameters, no further treatment was attempted.

\subsubsection{Activation energies}

Before comparing the activation energies determined in this work to theoretical calculations and other experiments, I want to briefly discuss them in light of basic concepts in reaction dynamics [98]. Based on the Bell-Evans-Polanyi principle [99, 100] we expect the highly exothermic $\mathrm{CO}$ oxidation to have a low activation energy. Hammond's postulate [101] states that the structure of the transition state tends to resemble the reactants in an exothermic reaction and the products in an endothermic raction. In an early barrier reaction the transition state and the reactants are closely related in energy and thus there is little structural reorganization between them. This agrees well with the MEP calculation in figure 8.3.

The different dynamics strongly suggest two different transition states for the reaction on terraces (tt) and the reaction on steps (ss). Assuming both of them are early barrier reactions, the stabilization of the respective transition state is closely related to the stabilization of the reactants on their binding sites. If the energy of the transition state follows the reactants' energy, the relative activation energies should be similar. This agrees well with our experimental findings for the reaction on terraces $\left(\mathrm{E}_{\mathrm{a}}^{\mathrm{tt}}=(0.6 \pm 0.1) \mathrm{eV}\right)$ and the reaction on steps $\left(\mathrm{E}_{\mathrm{a}}^{\mathrm{ss}}=(0.65 \pm 0.1) \mathrm{eV}\right)$. 


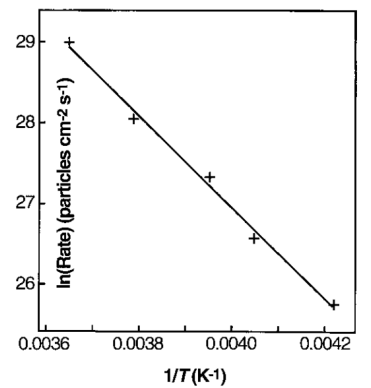

Wintterlin et al. [88]
If we further assume the ts- and the ss-reaction to access the same transition state via two different reactant geometries, we can intuitively explain the difference in activation energy between them: The step bound $\mathrm{CO}$ is stabilized by additional $0.16-0.35 \mathrm{eV}$ on step sites compared to $\mathrm{CO}$ on terrace sites $[45,57,58]$. If the reactants access the same transition state, the activation energy for the ts-reaction would be lowered by the same amount. The activation energy for the tsreaction would then be in the range of $0.30-0.49 \mathrm{eV}$. This agrees well with the experimentally determined value of $E_{a}^{t s}=(0.4 \pm 0.1) \mathrm{eV}$

Lastly, I want to compare the activation energies for the three different reactions determined in this work to other reported values. The activation energy with both reactants on terraces, $E_{a}^{t t}$, was determined as $(0.6 \pm 0.1) \mathrm{eV}$. This value is slightly lower than the DFT-RPBE calculation by Eichler [102] (0.73 eV) or the DFT-GGA MEP calculation also using the RPBE functional by Jan Altschäffel (o.8 eV) in figure 8.3.

A comparison with reported experimental values is far more challenging because it remains unclear which channels were observed in other studies. In 1992 Szabo, Henderson and Yates [103] investigated the structure sensitivity of the reaction in an isotope experiment and reported that $\mathrm{CO}$ on terrace sites is the reactive species at low temperatures $(<200 \mathrm{~K})$. This suggests that the activation energy of $0.49 \mathrm{eV}$ reported by Wintterlin et al. [88] in an STM experiment at $247 \mathrm{~K}$ is also due to reaction on terraces. Considering the difference in temperature and the fact that Wintterlin et al. reported the reaction to take place at island perimeters, it remains unclear whether we sample the same reaction pathway.

Nonetheless I want to compare the rate coefficient by Wintterlin et al. to our results. The report contains the microscopic reaction rate coefficient. In order to compare with our macroscopic rate coefficients we convert Wintterlin's data by dividing the reported rate by the concentration of $\mathrm{O}$ atoms on the surface, as described in the report [88]. The maximum concentration of O-atoms on a $\mathrm{Pt}(111)$ surface at a coverage of $\theta_{\max }=0.25 \mathrm{ML}$ is $3.75 \times 10^{14}$ particles $/ \mathrm{cm}^{2}$. As the actual concentration of O-atoms in Wintterlin's experiment is unknown, I estimate three probable coverages $\left(\theta_{\max }, 1 / 2 \theta_{\max }\right.$ and $\left.1 / 4 \theta_{\max }\right)$. The conversion for these three coverages is compared to our experimental results in figure 8.5.

The rate coefficients by Wintterlin et al. agree surprisingly well with our terrace-terrace $(\mathrm{tt})$ reaction rate coefficient. Note that our rate coefficient was extrapolated over more than $300 \mathrm{~K}$. Keep in mind that claiming the low temperature reaction channel to be the same as our tt-reaction solely based on the report by Szabo et al. [103] is quite speculative. 


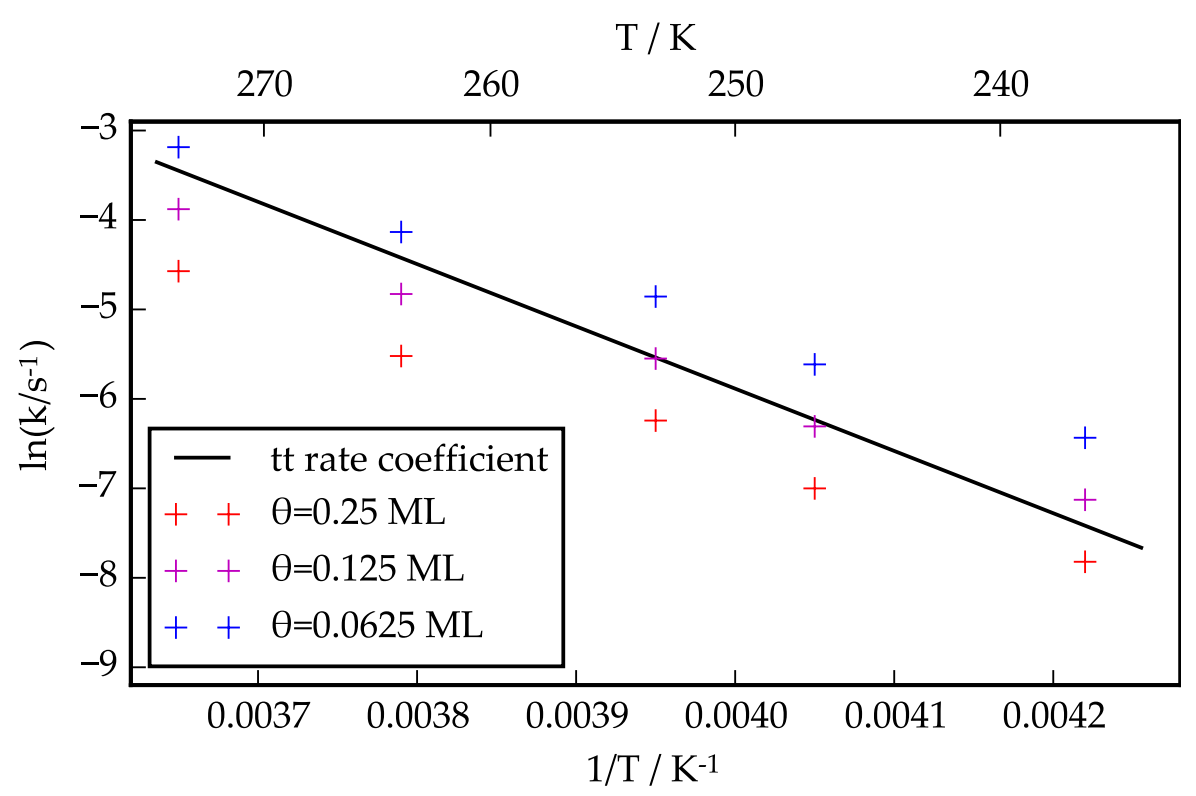

Figure 8.5: Comparison of the tt-reaction rate coefficient from this work (black line) to the rate coefficient by Wintterlin et al. [88]. Different O-atom coverages were used to convert the data, see legend and text.

\subsection{MODELING APPROACH}

The treatment of the kinetic traces in this work follows a LH-type model. The reaction scheme in section 7.1.1 is based on typical assumptions for a LH model [68] and some assumptions specific to this reaction.

1. There are two kinds of adsorption sites on the surface, terrace sites $S_{t}$ and step sites $S_{s}$. All terrace sites are identical, and so are the step sites.

2. $\mathrm{CO}$ requires a free site upon adsorption, $\mathrm{O}_{2}$ dissociates upon adsorption and thus two $\mathrm{O}$-atoms need two sites. $\mathrm{CO}$ and $\mathrm{O}$ compete for the same sites.

3. Surface sites are liberated upon reaction and subsequent desorption or upon desorption of the reactants.

4. The molecules and atoms are in thermal equilibrium prior to the reaction and distributed randomly within their domain (terrace or steps).

5. Interaction between molecules on the surface are small enough not to influence the reaction.

It is well known that both $\mathrm{CO}$ and $\mathrm{O}$ bind differently to terrace or step sites [57-60], which supports the first assumption. The competition between $\mathrm{CO}$ and $\mathrm{O}$ for sites is a little more complicated and has 
been described in section 7.1.5. The conservation of sites expressed in assumption 2 and 3 is obvious.

Whether assumption 4 withstands critical examination is more difficult to answer. Oxygen islands on platinum terraces for example are known to dissolve above $300 \mathrm{~K}$ [104-106]. This is precisely the temperature where the mean translational energy $k_{B} T$ surpasses the attractive interactions of $25 \mathrm{meV}$ reported by Ohta et al. [104] and Wintterlin and Völkening [107].

Molecular oxygen from the incident beam is known to dissociate at temperatures as low as $140 \mathrm{~K}[87]$, the dissociation at the temperature range of this work must thus take place on a nanosecond timescale. The oxidation of $\mathrm{CO}$ has been measured with varying delays between the O-pulse and the following CO pulse - no differences were found. This suggests that oxygen is fully dissociated and a steady-state $\mathrm{O}-$ atom concentration is established before the $\mathrm{CO}$ pulse.

Whether the $\mathrm{O}$-atoms are randomly distributed is a different matter. The results of the kinetic model show that the preference for steps is smaller than expected for $\mathrm{Pt}(111)$. If this is an effect of significant amounts of $\mathrm{O}$-atoms being consumed at steps for each $\mathrm{CO}$ pulse, then some kind of $\mathrm{O}$-atom concentration gradient might be present across terraces. Estimating the effect of such a gradient would require a far more complex kinetic diffusion model involving a distribution of different terrace sizes. As $\mathrm{CO}$ is believed to be quite mobile on terraces, a $\mathrm{CO}$ molecule would see the average $\mathrm{O}$-atom concentration across its terrace. As long as the different terraces have comparable O-atom concentrations we would expect the effect to be small.

The attractive interactions between oxygen atoms have been reported to be as low as $5.8 \mathrm{meV}$ by Tang et al. [105] or $25 \mathrm{meV}$ by Ohta [104] and Wintterlin [107]. This value is too small to have a significant effect on the reaction as it is below the translational energy of $k_{B} T=53 \mathrm{meV}$ at $T_{s}=600 \mathrm{~K}$. The interaction between two $\mathrm{CO}$ molecules can be neglected due to the small concentrations of $\mathrm{CO}$ on the surface, the interaction between $\mathrm{O}$ atoms and $\mathrm{CO}$ remains unknown.

When fitting analytical (pseudo first-order reaction) equations to the kinetic traces, additional assumptions must be made:

6. The concentration of $\mathrm{O}$ atoms on terraces and steps is constant for the duration of the $\mathrm{CO}$ pulse.

7. The concentration of free terrace and step sites must also be constant.

Calculating the kinetic trace numerically allows us to avoid assumptions 6 and 7 and is therefore the more flexible approach. The computational time for each execution of the numeric solution is very short, which allows us to pass it to optimization routines. By changing a few key parts of the python code the numeric modeling can be applied to any future reaction carried out at Beamer II. 
The application of ion imaging to study processes at surfaces developed at the Beamer II apparatus has proven to be a very powerful experimental technique. It allows us to measure the speed and angular distribution in a single measurement. As each ion image we record is velocity-resolved this also applies to kinetic scans of reaction products or the incident beam. We thus calculate the time-of-flight for both incident beam and product molecules and obtain the product flux leaving the surface as a function of reaction time on the surface, the kinetic trace.

Most metal surfaces possess different sites with distinct activity. Most experiments actually measure a combination of the reactions at different sites. Unless the flux from the different reaction channels can be distinguished by a dynamical fingerprint this prevents thorough mechanistic understanding. For systems where different sites produce product flux with different velocities, ion imaging allows us to measure the reaction channels individually and simultaneously.

This technique requires only one laser and the strong-field ionization can detect any molecule. Compared to the tagging technique by Golibrzuch et al., which requires a long-lived, suitable excited state, the general applicability of the techniques used at the Beamer II experiment is a significant advantage.

Another benefit of the imaging method is that it provides the researcher with an on-the-fly visual representation of the ion distributions and velocities during the experiment. This helps to discriminate the product flux from background signal or the incoming beam, and space-charge effects can be recognized immediately.

Furthermore we compared a REMPI wavelength scan taken by integrating an ROI from the imaging detector with one taken using the pure MCP signal and found the SNR of the imaging-detected REMPI spectrum to be superior. As we can choose a region of interest from the ion images the noise outside of this region is discarded immediately.

Lastly, despite the many advantages and the comfortable use of single laser ionization the technique remains experimentally challenging. Especially the electric fields need to be well defined and thus require flat grids. The preparation and handling of the grids requires some experience. 


\subsection{IMPACT OF THE RESULTS}

Using our kinetic model and the determined rate coefficients we can simulate the oxidation probability of $\mathrm{CO}$ adsorbed on $\mathrm{Pt}(111)$ as a function of temperature and O-atom coverage. The result of such a simulation is shown in figure 8.6.

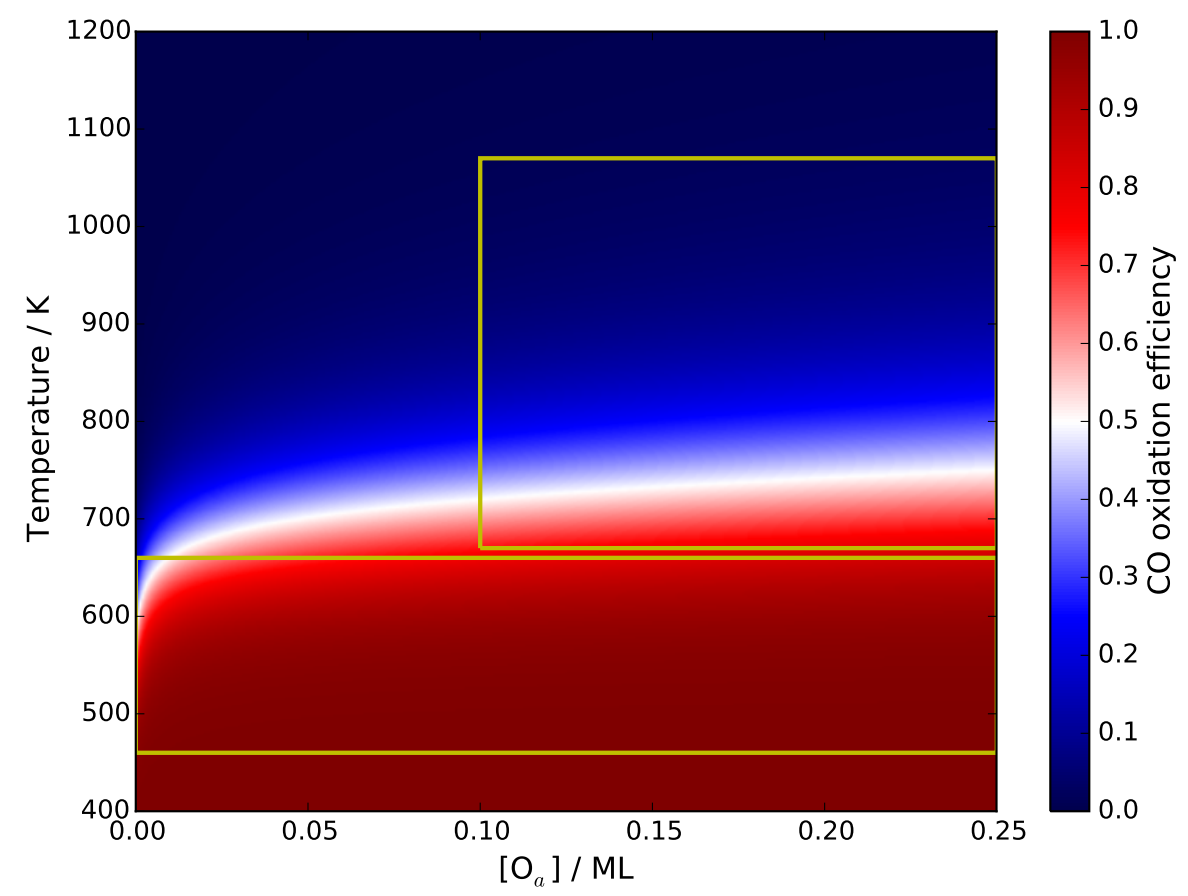

Figure 8.6: $\mathrm{CO}$ oxidation efficiency on $\mathrm{Pt}(111)$ vs temperature and $\mathrm{O}$-atom coverage. Reproduced with permission from Ref. [46].

On a first glance we see that the oxidation probability is close to unity at low temperatures and decreases rapidly at temperatures higher than $700 \mathrm{~K}$. The desorption rate coefficient of $\mathrm{CO}$ has a large activation energy and thus increases strongly with temperature. The increase in reaction rates does not compensate this strong increase in desorption rate. The lower yellow rectangle shows the region where this work and previous investigations were conducted. The second rectangle at higher temperatures denotes the range where $\mathrm{CO}$ oxidation in car catalysts takes place.

Extensive empirical studies on the optimal catalyst conditions for $\mathrm{CO}$ oxidation have been carried out by the automobile industry already. No further improvements in this field can be expected from the revised rate coefficients and the reaction mechanism we determined. The $\mathrm{CO}$ oxidation efficiency of modern car catalysts is very high and further improvement efforts deal with other challenges such as $\mathrm{NO}_{x}$ reduction. From careful examination of the conversion rates we can learn a great deal about the rules governing catalytic activity though.

Figure 8.7 shows the hyperthermal fraction of the total $\mathrm{CO}_{2}$ flux vs $\mathrm{O}$-atom coverage and temperature. The thermal $\mathrm{CO}_{2}$ dominates 


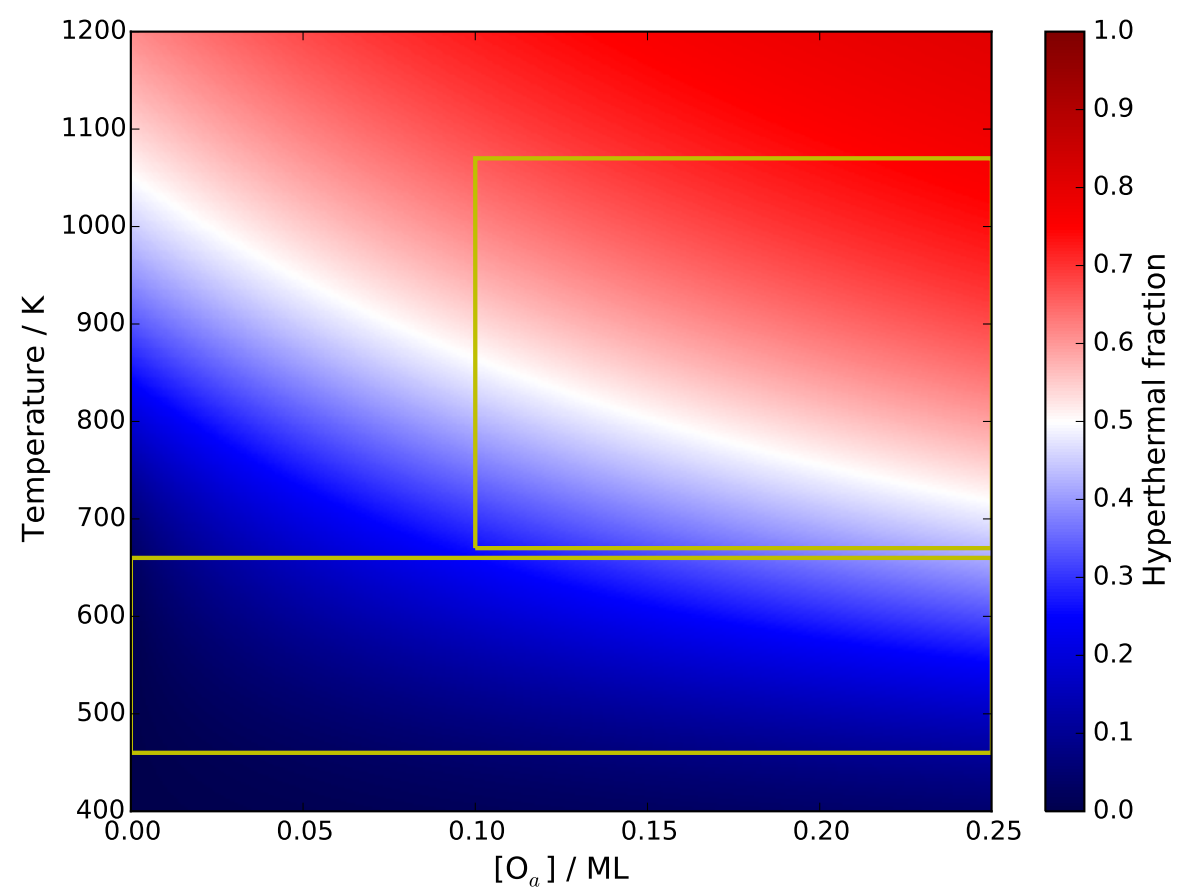

Figure 8.7: Hyperthermal fraction of the total $\mathrm{CO}_{2}$ flux vs temperature and O-atom coverage. Reproduced with permission from Ref. [46].

at low temperatures and at low $\mathrm{O}$-atom coverages. The hyperthermal fraction dominates at higher temperatures and high $\mathrm{O}$-atom coverages. As the activation energies of the TT and the SS reaction $(0.6 \mathrm{eV}$ and $0.65 \mathrm{eV}$ ) are rather similar the abundance of hyperthermal $\mathrm{CO}_{2}$ at high temperatures can at first be surprising. From figure 8.6 we learned that reaction and desorption compete at high temperatures. We can now combine these two observations and conclude that the desorption is much faster than the diffusion at high temperatures. Either $\mathrm{CO}$ on the hot platinum surface reacts very quickly or it desorbs - it does not stay on the surface long enough to diffuse over considerable distances. The initial sticking determines the reaction site at high temperatures. The majority site thus determines the reactivity at high temperatures.

Nørskov et al. have described a similar concept in their seminal 2008 paper [108]: In order to compare the relative importance of different catalytic sites on a surface one should compare the geometry probability-weighted activation energies $E_{a, i}^{w}$,

$$
\mathrm{E}_{\mathrm{a}, \mathrm{i}}^{w}=\mathrm{E}_{\mathrm{a}, \mathrm{i}}-\mathrm{k}_{\mathrm{B}} \mathrm{T} \ln \mathrm{A}_{\mathrm{i}},
$$

where $A_{i}$ and $E_{a, i}$ are the abundance and the activation energy of site i. If $E_{a, i}^{w}$ for one active site is smaller by at least a factor of $k_{B} T$, this site is expected to dominate the catalytic activity [108].

This description compares elementary step reactions under the assumption that the distribution of reactants over the different sites matches the abundance of the respective site. For the catalytic oxida- 
Equilibrium can be troublesome in molecular beam experiments. tion of $\mathrm{CO}$ on platinum this assumption is not met for a large range of experimental conditions. At low temperatures the adsorbates clearly prefer the stronger binding to step sites, which can even cause catalytic poisoning. At high temperatures the diffusion is too slow for the adsorbates to establish an equilibrium between the different sites. The initial sticking depends on the geometric abundance, so the reactions are determined by the site abundance. But the catalytic activity is not related to the activation energy of the different catalytic sites anymore - simply because the adsorbates do not even reach the step sites prior to desorption.

As stated in the introduction one of the essential goals for any experimentalist in this field should be to conduct experiments which measure clearly defined properties and thus compare well to theoretical predictions. The experiment should thus be carried out on the simplest system possible and the reported properties should ideally describe a simple process such as an elementary step reaction.

Transition state theory (TST) is often considered the method of choice to describe reaction rate coefficients and thus predict chemical reactivity and catalytic activity. The procedures for predicting the rate coefficients of gas-phase reactions are well understood, even though there can be complications when the assumption of equilibrium [109] between reactants and activated transition state complexes is not fulfilled.

For processes such as a Langmuir-Hinshelwood reaction on a surface with thermalized reactants this equilibrium condition should be fulfilled. In case the other conditions of TST apply to the elementary reaction step, one can use the experimentally determined reaction rate coefficients to investigate the proper method to do a TST prediction.

The rate coefficient in TST is defined as

$$
k_{\mathrm{TST}}=\frac{k_{\mathrm{B}} T}{h} \exp \left(-\frac{\Delta^{\ddagger} \mathrm{G}}{\mathrm{k}_{\mathrm{B}} \mathrm{T}}\right) .
$$

The temperature dependent Gibbs energy of activation $\Delta^{\ddagger} \mathrm{G}$ in equation 8.11 can substituted using the Gibbs-Helmholtz equation leading to

$$
\mathrm{k}_{\mathrm{TST}}=\frac{\mathrm{k}_{\mathrm{B}} \mathrm{T}}{\mathrm{h}} \exp \left(\Delta^{\ddagger} \mathrm{S} / \mathrm{k}\right) \exp \left(-\frac{\Delta^{\ddagger} \mathrm{H}}{\mathrm{k}_{\mathrm{B}} \mathrm{T}}\right) .
$$

In analogy to the Arrhenius equation the entropy term $\exp \left(\Delta^{\ddagger} S / k\right)$ determines the preexponential factor and the enthalpy difference $\Delta^{\ddagger} \mathrm{H}$ corresponds to the activation energy.

Based on statistical mechanics the entropy change from reactants to the transition state $\Delta^{\ddagger} S$ can be expressed by the partition function at the transition state $Q_{\ddagger}$ (excluding the reaction coordinate) and the 
partition functions $\mathrm{Q}_{\mathrm{CO}}$ and $\mathrm{Q}_{\mathrm{O}}$ of the reactants $\mathrm{CO}$ and $\mathrm{O}$. This transformation leads to

$$
\mathrm{k}_{\mathrm{TST}}=\frac{\mathrm{k}_{\mathrm{B}} \mathrm{T}}{\mathrm{h}} \frac{\mathrm{Q}_{\ddagger}}{\mathrm{Q}_{\mathrm{CO}} \mathrm{Q}_{\mathrm{O}}} \exp \left(-\frac{\mathrm{E}_{\mathrm{O}}}{\mathrm{k}_{\mathrm{B}} \mathrm{T}}\right),
$$

with the energy difference of reactants and transition state at absolute zero $E_{0}$. This is the definition as reported by Eyring in 1935 [109].

A fairly common technique to determine the energy barrier to the transition state is a minimum energy pathway calculation, as has been shown in figure 8.3 for $\mathrm{CO}$ and $\mathrm{O}$ on $\mathrm{Pt}(111)$ terraces. While the choice of the DFT functional will affect the obtained barrier height, the strengths and weaknesses of this approach are quite well understood.

The entropy of the adsorbates can be described in different ways. One limiting case is to treat the adsorbates as immobile and approximate the translation as a set of vibrations. Treating the translation like a harmonic oscillator results in a very low entropy difference $\Delta^{\ddagger} S$, i. e. small partition functions $Q_{c o}$ and $Q_{O}$ of the reactants. A calculation for the CO oxidation on $\mathrm{Pt}(111)$ terraces using this harmonic approximation has been reported by Eichler [102]. Our experimentally determined values are shown in table 8.2 on page 138 .

While the activation energy of $0.74 \mathrm{eV}$ by Eichler is not that different from our experimentally determined activation energy for the reaction on terraces of $0.6 \mathrm{eV}$, the harmonic approximation by Eichler overestimates the prefactor $\left(5 \times 10^{12} \mathrm{~s}^{-1}\right)$ by several orders of magnitude compared. The prefactor we determined for the tt-reaction $\left(3.5 \times 10^{9} \mathrm{~s}^{-1}\right)$ is significantly lower.

A comparison of the rate coefficients is shown in figure 8.8. The result by Eichler is plotted as a grey line.

The harmonic approximation, which gives an upper limit to the reaction rate, clearly fails to reproduce the experimentally observed reaction rate coefficients. The barrier height reported by Eichler was calculated using DFT and the RPBE functional, which is known to overestimate reaction barriers [110]. However, a lower activation energy would only worsen the discrepancy. We can thus conclude that while the approach is very promising, too little effort was spent on properly determining the entropy terms of the reactants and the transition state.

A more elaborate investigation of the adsorbate entropies was recently reported by Jørgensen and Grönbeck [111]. The adsorbate entropy was calculated using four different approximations: Immobile adsorbates with the harmonic approximation as used by Eichler yielded the lowest adsorbate entropy and thus the largest prefactor. The highest entropy change was obtained when treating the adsorbates as freely translating $2 \mathrm{D}$ gas. A hindered translator model and the complete potential energy sampling (CPES) approach resulted in values 


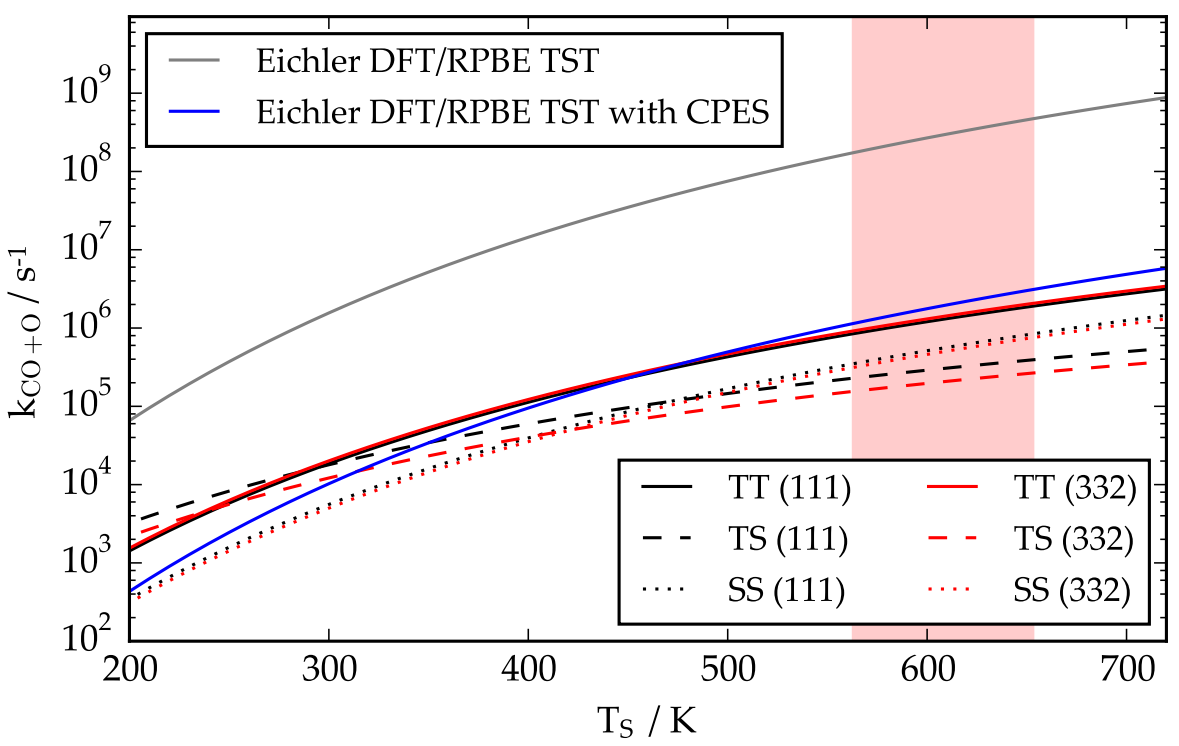

Figure 8.8: Comparison of TST and experimental values. The temperature range probed in this work is highlighted in red.

in between. The prefactor obtained from the CPES calculation combined with the activation energy as calculated by Eichler is plotted as blue line in figure 8.8. The agreement with the rate constant on terraces is quite good; an accurate description of the reactivity using TST is possible when using the right methods.

When comparing different DFT functionals [110] Diaz et al. stated that the only way to distinguish which functional is more suitable is through a comparison with suitable experimental results, with a meaningful comparison requiring a deep knowledge of the experimental details. From the discussion above we learn that this equally applies when comparing TST approximations.

The profound information we acquired during the investigation presented in this work allowed us to deduce the reaction mechanism and report active-site selected elementary step reaction rate coefficients. With such a clear assignment the comparison of reactions at terrace sites to theoretical results becomes straightforward and more meaningful. Additionally, this work prompts researchers to extend the theoretical simulation to cover reaction at step sites and provides reaction rate coefficients for comparison. Lastly, whether the theory can reproduce the very different dynamical fingerprints of reactions at terrace or step sites should be a strong indication of the success of the method.

In summary, this work reports clearly assigned experimental results which will serve as a benchmark to further improve theoretical understanding of reactions at surfaces. The measurement of the comprehensive data leading to these results was only possible through velocity-resolved kinetics. The oxidation of $\mathrm{CO}$ on platinum turned out to be an excellent candidate to test the potential of this new 
method. While the experimental setup is challenging, the advantages of ion imaging detectors for the study of chemical kinetics and dynamics at surfaces are convincing - the new method presented in this work is already being implemented in several new experiments.

\section{REFERENCES}

[37] George Comsa and Rudolf David. "Dynamical parameters of desorbing molecules." In: Surface Science Reports 5.4 (Dec. 1985), pp. 145-198. DOI: 10 . 1016/0167 - 5729 (85 ) 90009 - 3 (cit. on pp. $45,46,50,51,131$ ).

[45] Dmitriy Borodin. "Probing Reactions at Surfaces using Ion Imaging - CO Oxidation at Atomically Flat and Stepped Surfaces of Platinum and Palladium." Master Thesis. Georg-AugustUniversity Goettingen, 2017 (cit. on pp. 71, 142).

[46] Jannis Neugebohren, Dmitriy Borodin, Hinrich W. Hahn, Jan Altschäffel, Alexander Kandratsenka, Daniel J. Auerbach, Charles T. Campbell, Dirk Schwarzer, Dan J. Harding, Alec M. Wodtke, and Theofanis N. Kitsopoulos. "Velocity-resolved kinetics of site-specific carbon monoxide oxidation on platinum surfaces." In: Nature 558.7709 (June 2018), pp. 280-283. Dor: 10.1038 / s41586-018-0188-x (cit. on pp. 79-82, 123, 130, 132, 134, 146, 147).

[47] E. Poehlmann, M. Schmitt, H. Hoinkes, and H. Wilsch. "Velocity distributions of carbondioxide molecules from the oxidation of CO on Pt(111)." In: Surface Science 287-288 (May 1993), pp. 269-272. DOI: 10 . 1016/0039 - 6028 ( 93 ) 90784 - H (cit. on pp. 80, 81, 129, 131).

[48] K.-H. Allers, H. Pfnür, P. Feulner, and D. Menzel. "Fast Reaction Products from the Oxidation of CO on Pt(111): Angular and Velocity Distributions of the $\mathrm{CO}_{2}$ Product Molecules." In: The Journal of Chemical Physics 100.111 (1994), pp. 3985-3998. DOI: 10.1063/1. 466332 (cit. on pp. 80, 81, 129, 131, 133, 136).

[49] J. Segner, C. T. Campbell, G. Doyen, and G. Ertl. “Catalytic oxidation of $\mathrm{CO}$ on $\mathrm{Pt}(111)$ : The influence of surface defects and composition on the reaction dynamics." In: Surface Science 138.2-3 (Mar. 1984), pp. 505-523. DOI: 10. 1016/0039-6028 (84) 90262- 0 (cit. on pp. 81, 88, 96, 129, 135-137).

[50] E. Poehlmann, M. Schmitt, H. Hoinkes, and H. Wilsch. "Bimodal angular and velocity distributions of $\mathrm{CO}_{2}$ desorbing after oxidation of CO on Pt (111)." In: Surface Review and Letters 02.06 (Dec. 1995), pp. 741-758. DOI: 10.1142/S0218625X95000674 (cit. on pp. 81, 129, 133). 
[53] Charles A. Becker, James P. Cowin, Lennard Wharton, and Daniel J. Auerbach. " $\mathrm{CO}_{2}$ product velocity distributions for CO oxidation on platinum." In: The Journal of Chemical Physics 67.7 (1977), p. 3394. DOI: 10.1063/1. 435289 (cit. on pp. 87, 131, 135).

[54] Robert L. Palmer and Joe N. Jr Smith. "Molecular beam study of CO oxidation on a (111) platinum surface." In: The Journal of Chemical Physics 60.4 (1974), p. 1453. DOI: 10.1063/1. 1681219 (cit. on pp. 87, 129, 135).

[55] C. T. Campbell, G. Ertl, H. Kuipers, and J. Segner. "A Molecular Beam Study of the Catalytic Oxidation of $\mathrm{CO}$ on a $\mathrm{Pt}(111)$ Surface." In: The Journal of Chemical Physics 73.111 (1980), pp. 58625873. DOI: 10.1063/1. 440029 (cit. on pp. 87, 135, 136).

[57] J. E. Reutt-Robey, D. J. Doren, Y. J. Chabal, and S. B. Christman. "Microscopic CO Diffusion on a Pt(111) Surface by TimeResolved Infrared Spectroscopy." In: Physical Review Letters 61.24 (May 1988), pp. 2778-2781. DoI: 10 . 1103 / PhysRevLett . 61. 2778 (cit. on pp. 90, 139, 142, 143).

[58] T. H. Lin and G. A. Somorjai. "Modulated molecular beam scattering of $\mathrm{CO}$ and $\mathrm{NO}$ from $\mathrm{Pt}(111)$ and the stepped $\mathrm{Pt}(557)$ crystal surfaces." In: Surface Science 107.2-3 (1981), pp. 573-585. DOI: 10 . 1016/0039 - 6028 (81) 90546 - X (cit. on pp. 90, 137, 142, 143).

[59] Ryosuke Jinnouchi, Kensaku Kodama, and Yu Morimoto. “DFT calculations on $\mathrm{H}, \mathrm{OH}$ and $\mathrm{O}$ adsorbate formations on $\mathrm{Pt}(111)$ and $\mathrm{Pt}(332)$ electrodes." In: Journal of Electroanalytical Chemistry 716 (Mar. 2014), pp. 31-44. DoI: 10.1016/j . j elechem. 2013.09. 031 (cit. on pp. 90, 112, 125, 143).

[6o] A. Nagoya, Ryosuke Jinnouchi, Kensaku Kodama, and Yu Morimoto. "DFT calculations on $\mathrm{H}, \mathrm{OH}$ and $\mathrm{O}$ adsorbate formations on $\mathrm{Pt}$ (322) electrode." In: Journal of Electroanalytical Chemistry 757 (Nov. 2015), pp. 116-127. DOI: 10.1016/ j . jelechem . 2015.09.009 (cit. on pp. 90, 112, 125, 137, 143).

[67] John L. Gland and Edward B. Kollin. "Vibrational characterization of carbon monoxide oxidation on the Pt(111) surface." In: Surface Science 151.1 (1985), pp. 260-270. DOI: 10 . 1016/0039 6028 ( 85 ) $90465-0$ (cit. on pp. 100, 136).

[68] J. Liu, M. Xu, and F. Zaera. "Determination of the rate limiting step in the oxidation of $\mathrm{CO}$ on $\operatorname{Pt}(111)$ surfaces." In: Catalysis Letters 37.1-2 (1996), pp. 9-13. DOI: 10 . 1007 / BF00813512 (cit. on pp. 100, 143). 
[71] Bene Poelsema, Laurens K. Verheij, and George Comsa. "HeScattering Investigation of CO Migration on Pt(111)." In: Physical Review Letters 49.23 (Dec. 1982), pp. 1731-1735. DOI: 10. 1103/PhysRevLett. 49.1731 (cit. on pp. 114, 139, 140).

[72] Robert L Palmer. "Molecular beam study of oxygen and $C_{2}$ hydrocarbon chemisorption and reactions on Pt(111)." In: Journal of Vacuum Science and Technology 12.6 (Nov. 1975), pp. 14031409. DOI: 10.1116/1. 568551 (cit. on p. 129).

[73] Robert L. Palmer and Joe N. Smith. "Recent Studies of Chemical Interactions on Surfaces Using Molecular Beams." In: Catalysis Reviews 12.1 (1975), pp. 279-301. DOI: 10/cbps2k (cit. on p. 129).

[74] P.R. Norton. "Surface analysis of platinum by x-ray photoelectron spectroscopy (XPS)." In: Surface Science 44.2 (Aug. 1974), pp. 624-628. DOI: 10 . 1016/0039-6028 (74 ) 90142 - 3 (cit. on p. 131).

[75] H. Davy. "On the Fire-Damp of Coal Mines, and on Methods of Lighting the Mines So as to Prevent Its Explosion." In: Philosophical Transactions of the Royal Society of London 106.January (1816), pp. 1-22. DoI: 10.1098/rstl . 1816.0001 (cit. on p. 135).

[76] W. Henry. "XLIV. On the action of finely-divided platinum on gaseous mixtures, and its application to their analysis." In: Philosophical Magazine Series 1 65.May (1825), pp. 269-283. DoI: 10.1080/14786442508628435 (cit. on p. 135).

[77] Irving Langmuir. "Chemical reactions at very low pressures. I. The clean-up in a tungsten lamp." In: Journal of the American Chemical Society 35.2 (Feb. 1913), pp. 105-127. DOI: 10.1021/ ja02191a001 (cit. on p. 135).

[78] Irving Langmuir. "Chemical reactions at very low pressures. II. The chemical clean-up of nitrogen in a tungsten lamp." In: Journal of the American Chemical Society 1310 (1913). Dor: 10 . 1021/ja02197a002 (cit. on p. 135).

[79] Irving Langmuir. "Surface Chemistry." In: Chemical Reviews 13.2 (Oct. 1933), pp. 147-191. DOI: 10.1021/ cr60045a001 (cit. on p. 135).

[8o] Irving Langmuir. "The mechanism of the catalytic action of platinum in the reactions $2 \mathrm{CO}+\mathrm{O}_{2}=2 \mathrm{CO}_{2}$ and $2 \mathrm{H}_{2}+\mathrm{O}_{2}=$ ${ }_{2} \mathrm{H}_{2} \mathrm{O}$." In: Transactions of the Faraday Society 17.1905 (1922), p. 621. DOI: 10.1039/tf9221700621 (cit. on p. 135).

[81] F. L. Hughes and Henry Levinstein. "Mean Adsorption Lifetime of $\mathrm{Rb}$ on Etched Tungsten Single Crystals: Neutrals." In: Physical Review 113.4 (Feb. 1959), pp. 1036-1038. Dor: 10.1103/ PhysRev.113.1036 (cit. on p. 135). 
[82] T. Engel and G. Ertl. "Elementary Steps in the Catalytic Oxidation of Carbon Monoxide on Platinum Metals." In: Advances in Catalysis. Vol. 28. 1979, pp. 1-78. DoI: 10. 1016/S0360-0564 ( 08 ) 60133- 9 (cit. on p. 135).

[83] H. P. Bonzel and R. Ku. "Carbon Monoxide Oxidation on a $\mathrm{Pt}(110)$ Single Crystal Surface." In: Journal of Vacuum Science and Technology 9.2 (Mar. 1972), pp. 663-667. DoI: 10.1116/1. 1317748 (cit. on p. 135).

[84] H. P. Bonzel and R. Ku. "Mechanisms of the catalytic carbon monoxide oxidation on Pt (110)." In: Surface Science 33.1 (Oct. 1972), pp. 91-106. DOI: 10 . 1016/0039 - 6028 (72 ) 90101 - X (cit. on p. 135).

[85] G Ertl. "Heterogeneous catalysis on the atomic scale." In: Chemical record (New York, N.Y.) 1.1 (2001), pp. 33-45. DOI: 10.1016/ S1381-1169 (01)00460-5 (cit. on p. 135).

[86] John L. Gland and Edward B. Kollin. "Carbon monoxide oxidation on the $\operatorname{Pt}(111)$ surface: Temperature programmed reaction of coadsorbed atomic oxygen and carbon monoxide." In: J. Chem. Phys. 78.2 (1983), p. 963. DoI: 10.1063/1 . 444801 (cit. on p. 136).

[87] Sohail Akhter and J. M. White. "The effect of oxygen islanding on $\mathrm{CO}$ and $\mathrm{H}_{2}$ oxidation on $\mathrm{Pt}(111)$." In: Surface Science 171.3 (1986), pp. 527-542. DOI: 10.1016/0039-6028 (86) 91058 - 7 (cit. on pp. 136, 144).

[88] J. Wintterlin, S. Völkening, T. V. W. Janssens, T. Zambelli, and G. Ertl. "Atomic and Macroscopic Reaction Rates of a SurfaceCatalyzed Reaction." In: Science 278.5345 (Dec. 1997), pp. 19311934. DOI: 10.1126/science.278.5345. 1931 (cit. on pp. 136, 142, 143).

[89] Jiazhan $\mathrm{Xu}$ and John T. Yates. "Catalytic oxidation of $\mathrm{CO}$ on $\mathrm{Pt}$ (335): A study of the active site." In: The Journal of Chemical Physics 99.1 (July 1993), pp. 725-732. DOI: 10.1063/1. 465745 (cit. on p. 137).

[90] Thomas Engel and Philip Reid. Physikalische Chemie. 1st ed. München: Pearson Education, 2006, pp. 1024-1025 (cit. on p. 138).

[91] Paul L. Houston. Chemical Kinetics and Reaction Dynamics. Mineola, New York: Dover Publications, Inc., 2001, pp. 183-185 (cit. on p. 138).

[92] V. J. Kwasniewski and L. D. Schmidt. "Surface diffusion of CO on Pt(111)." In: Surface Science 274.3 (Aug. 1992), pp. 329-340. DOI: 10.1016/0039-6028(92) 90838-W (cit. on p. 139). 
[93] C. M. Yim, K. L. Man, Xudong Xiao, and M. S. Altman. "Lowenergy electron microscopy of $\mathrm{CO} / \mathrm{Pt}$ (111) surface diffusion by nonequilibrium coverage profile evolution." In: Physical Review B - Condensed Matter and Materials Physics 78.15 (2008), pp. 1-9. DOI: 10.1103/PhysRevB. 78.155439 (cit. on p. 139).

[94] J. E. Reutt-Robey, D. J. Doren, Y. J. Chabal, and S. B. Christman. "CO diffusion on $\mathrm{Pt}(111)$ with time-resolved infrared-pulsed molecular beam methods: Critical tests and analysis." In: The Journal of Chemical Physics 93.12 (Dec. 1990), pp. 9113-9129. Dor: 10.1063/1. 459202 (cit. on pp. 139, 140).

[95] Jianwei Ma, Xudong Xiao, N. J. DiNardo, and M. M. T. Loy. "Diffusion of CO on $\operatorname{Pt}(111)$ studied by an optical diffraction method." In: Physical Review B 58.8 (1998), pp. 4977-4983. DoI: 10.1103/PhysRevB. 58.4977 (cit. on pp. 139, 140).

[96] Kedong Wang, Fangfei Ming, Qiang Huang, Xieqiu Zhang, and Xudong Xiao. "Study of CO diffusion on stepped Pt(111) surface by scanning tunneling microscopy." In: Surface Science 604.3-4 (2010), pp. 322-326. DOI: 10. 1016/j . susc . 2009 . 11.024 (cit. on pp. 139, 140).

[97] Radek Erban, Jonathan Chapman, and Philip Maini. A practical guide to stochastic simulations of reaction-diffusion processes. 2007 (cit. on p. 141).

[98] John C. Polanyi. "Some Concepts in Reaction Dynamics." In: Science 236 (1987), pp. 680-690. Dor: 10.1126 / science. 236. 4802.680 (cit. on p. 141).

[99] Ronald P. Bell. "The Theory of Reactions Involving Proton Transfers." In: Proceedings of the Royal Society A: Mathematical, Physical and Engineering Sciences 154.882 (Apr. 1936), pp. 414429. DOI: 10.1098/rspa. 1936.0060 (cit. on p. 141).

[10o] M. G. Evans and M. Polanyi. "Inertia and driving force of chemical reactions." In: Transactions of the Faraday Society 34 (1938), p. 11. DOI: 10. 1039/tf9383400011 (cit. on p. 141).

[101] George S. Hammond. "A Correlation of Reaction Rates." In: Journal of the American Chemical Society 77.2 (1955), pp. 334-338. DOI: 10.1021/ja01607a027 (cit. on p. 141).

[102] Andreas Eichler. "CO oxidation on transition metal surfaces: reaction rates from first principles." In: Surface Science 498.3 (Feb. 2002), pp. 314-320. DOI: 10.1016/S0039-6028 (01) 01805 2 (cit. on pp. 142, 149).

[103] András Szabó, Michael A. Henderson, and John T. Yates. “Oxidation of $\mathrm{CO}$ by oxygen on a stepped platinum surface: Identification of the reaction site." In: The Journal of Chemical Physics 96.8 (1992), p. 6191. DOI: 10.1063/1. 462636 (cit. on p. 142). 
[104] Masanari Nagasaka, Hiroshi Kondoh, Ikuyo Nakai, and Toshiaki Ohta. "Oxygen island formation on $\mathrm{Pt}(111)$ studied by dynamic Monte Carlo simulation." In: The Journal of Chemical Physics 122.4 (Jan. 2005), p. 044715. DOI: 10.1063/1. 1835270 (cit. on p. 144).

[105] Hairong Tang, Anton Van der Ven, and Bernhardt L. Trout. "Phase diagram of oxygen adsorbed on platinum (111) by firstprinciples investigation." In: Physical Review B 70.4 (July 2004), p. 045420. DOI: 10.1103/PhysRevB. 70.045420 (cit. on p. 144).

[106] Joost Wintterlin. Private Communications. 2017 (cit. on p. 144).

[107] S. Völkening and J. Wintterlin. "CO oxidation on $\operatorname{Pt}(111)$ Scanning tunneling microscopy experiments and Monte Carlo simulations." In: The Journal of Chemical Physics 114.14 (Apr. 2001), pp. 6382-6395. DOI: 10.1063/1. 1343836 (cit. on p. 144).

[108] Jens K. Nørskov, Thomas Bligaard, Britt Hvolbæk, Frank AbildPedersen, Ib Chorkendorff, and Claus H. Christensen. "The nature of the active site in heterogeneous metal catalysis." In: Chemical Society Reviews 37.10 (2008), p. 2163. DOI: 10.1039/ b800260f (cit. on p. 147).

[109] Henry Eyring. "The Activated Complex in Chemical Reactions." In: The Journal of Chemical Physics 3.2 (1935), pp. 107-115. DoI: 10.1063/1.1749604 (cit. on pp. 148, 149).

[110] C. Díaz, R. A. Olsen, H. F. Busnengo, and G. J. Kroes. “Dynamics on six-dimensional potential energy surfaces for $\mathrm{H}_{2} / \mathrm{Cu}(111)$ : Corrugation reducing procedure versus modified shepard interpolation method and PW91 versus RPBE." In: Journal of Physical Chemistry C 114.25 (2010), pp. 11192-11201. DOI: 10. 1021/jp1027096 (cit. on pp. 149, 150).

[111] Mikkel Jørgensen and Henrik Grönbeck. "Adsorbate Entropies with Complete Potential Energy Sampling in Microkinetic Modeling." In: Journal of Physical Chemistry C 121.13 (2017), pp. 71997207. DOI: 10.1021/acs. jpcc. 6 b11487 (cit. on p. 149). 
Part III

BIBLIOGR APHY 

[1] Barry E. Smith. "Nitrogenase Reveals Its Inner Secrets." In: Science 297.5587 (Sept. 2002), pp. 1654-1655. DoI: $10.1126 /$ science. 1076659 (cit. on p. 3).

[2] Jan Willem Erisman, Mark A. Sutton, James Galloway, Zbigniew Klimont, and Wilfried Winiwarter. "How a century of ammonia synthesis changed the world." In: Nature Geoscience 1.10 (2008), pp. 636-639. DOI: 10.1038/ngeo325 (cit. on p. 3).

[3] Alwin Mittasch. Geschichte der Ammoniaksynthese. Weinheim: Verlag Chemie, 1951 (cit. on p. 3).

[4] Gerhard Ertl. "Elementarschritte bei der heterogenen Katalyse." In: Angewandte Chemie 102.11 (Nov. 1990), pp. 1258-1266. DOI: 10.1002/ange. 19901021108 (cit. on p. 3).

[5] Oliver Bünermann, Hongyan Jiang, Yvonne Dorenkamp, Alexander Kandratsenka, Svenja M. Janke, Daniel J. Auerbach, and Alec M. Wodtke. "Electron-hole pair excitation determines the mechanism of hydrogen atom adsorption." In: Science 350.6266 (2015), pp. 1346-1349. DoI: 10.1126/science. aad4972 (cit. on p. 4).

[6] Svenja M. Janke, Daniel J. Auerbach, Alec M. Wodtke, and Alexander Kandratsenka. "An accurate full-dimensional potential energy surface for $\mathrm{H}-\mathrm{Au}(111)$ : Importance of nonadiabatic electronic excitation in energy transfer and adsorption." In: Journal of Chemical Physics 143.12 (2015). DoI: 10 . 1063/1. 4931669 (cit. on p. 4).

[7] R. G. Musket, W. McLean, C. A. Colmenares, D. M. Makowiecki, and W. J. Siekhaus. "Preparation of atomically clean surfaces of selected elements: A review." In: Applications of Surface Science 10.2 (Jan. 1982), pp. 143-207. Dor: 10. 1016/0378-5963 (82) 90142- 8 (cit. on p. 10).

[8] William Hallowes Miller. Treatise on Crystallography. London: Cambridge University Press, 1839 (cit. on p. 10).

[9] Lise Meitner. "Über die beta-Strahl-Spektra und ihren Zusammenhang mit der gamma-Strahlung." In: Zeitschrift für Physik A Hadrons and Nuclei 11.August (1922), pp. 35-54. DoI: 10. 1007/BF01328399 (cit. on p. 10).

[10] Pierre Auger. "Sur les rayons $\beta$ secondaires produits dans un gaz par des rayons." In: Comptes Rendus 180 (1923), pp. 169171 (cit. on p. 10). 
[11] Michael Schwarzer. "Reaction Dynamics and Kinetics of CO Oxidation on $\operatorname{Pd}(111)$ using Ion Imaging." Bachelor Thesis. Georg-August-Universität Göttingen, 2017 (cit. on p. 10).

[12] Davis E. Lawrence, Noel C. MacDonald, Paul W. Palmberg, Gerald E. Riach, and Roland E. Weber. Handbook of Auger Electron Spectroscopy. 2nd ed. Eden Prairie, Minnesota: Physical Electronics Industries, 1976 (cit. on p. 11).

[13] Giacinto Scoles, Davide Bassi, and Udo Buck. Atomic and Molecular Beam Methods. Vol. 1. 1988 (cit. on p. 13).

[14] David L. Proctor, Daniel R. Albert, and H. Floyd Davis. "Improved piezoelectric actuators for use in high-speed pulsed valves." In: Review of Scientific Instruments 81.2 (2010). DOI: 10. 1063/1.3309777 (cit. on pp. 13, 14).

[15] D. Proch and T. Trickl. "A high-intensity multi-purpose piezoelectric pulsed molecular beam source." In: Review of Scientific Instruments 60.4 (1989), pp. 713-716. DOI: 10.1063/1.1141006 (cit. on p. 14).

[16] Alice F. Schmidt-May, Monika Grütter, Jannis Neugebohren, T. N. Kitsopoulos, Alec M. Wodtke, and Dan J. Harding. "Rotationally Resolved Vacuum Ultraviolet Resonance-Enhanced Multiphoton Ionization (VUV REMPI) of Acetylene via the $\tilde{\mathrm{G}}$ Rydberg State." In: The Journal of Physical Chemistry A 120.27 (July 2016), pp. 5399-5407. DOI: 10 . 1021/acs . jpca . 6 b02477 (cit. on p. 15).

[17] David W. Chandler and Paul L. Houston. "Two-dimensional imaging of state-selected photodissociation products detected by multiphoton ionization." In: The Journal of Chemical Physics 87.2 (1987), p. 1445. DOI: 10. 1063/1.453276 (cit. on p. 19).

[18] David H. Parker and André T. J. B. Eppink. "Photoelectron and photofragment velocity map imaging of state-selected molecular oxygen dissociation/ionization dynamics." In: The Journal of Chemical Physics 107.7 (1997), p. 2357. DoI: 10. 1063/1. 474624 (cit. on pp. 19, 21).

[19] Christoph R. Gebhardt, T. Peter Rakitzis, Peter C. Samartzis, Vlassis Ladopoulos, and Theofanis N. Kitsopoulos. "Slice imaging: A new approach to ion imaging and velocity mapping." In: Review of Scientific Instruments 72.10 (Oct. 2001), pp. 38483853. DOI: 10.1063/1.1403010 (cit. on pp. 19, 26).

[20] D. J. De Rosier and A. Klug. "Reconstruction of three dimensional structures from electron micrographs." In: Nature 217.5124 (Jan. 1968), pp. 130-134. DOI: 10.1038/217130a0 (cit. on p. 19).

[21] M. Zwick and E. Zeitler. "Image Reconstruction from Projections." In: Optik 38 (1973), pp. 550-565 (cit. on p. 19). 
[22] B. Whitaker. Imaging in Molecular Dynamics. Ed. by Benjamin J. Whitaker. Cambridge: Cambridge University Press, 2003. DoI: 10. 1017/СB09780511535437 (cit. on pp. 21, 26, 35).

[23] Nils Henrik Abel. "Auflösung einer mechanischen Aufgabe." In: Journal für die reine und angewandte Mathematik 1 (1826), pp. 153-157 (cit. on p. 22).

[24] Dan J. Harding, Jannis Neugebohren, M. Grütter, A. F. SchmidtMay, D. J. Auerbach, T. N. Kitsopoulos, and A. M. Wodtke. "Single-field slice-imaging with a movable repeller: Photodissociation of $\mathrm{N}_{2} \mathrm{O}$ from a hot nozzle." In: The Journal of Chemical Physics 141.5 (Aug. 2014), p. 054201. DoI: 10.1063/1. 4891469 (cit. on pp. 26, 29, 33, 34).

[25] Daniel W. Kohn, Horst Clauberg, and Peter Chen. "Flash pyrolysis nozzle for generation of radicals in a supersonic jet expansion." In: Review of Scientific Instruments 63.8 (1992), pp. 40034005. DOI: 10.1063/1. 1143254 (cit. on p. 30).

[26] Ming Wu, David P. Taylor, and Philip M. Johnson. "Resonance enhanced multiphoton ionization photoelectron spectra of $\mathrm{CO}_{2}$. II. Competition between photoionization and dissociation." In: The Journal of Chemical Physics 95.2 (1991), p. 761. Dor: 10.1063/ 1.461082 (cit. on p. 30).

[27] Barry L. Lutz. "Presscure-Induced a" ${ }^{1} \Sigma_{g}^{+} \leftarrow X^{1} \Sigma_{g}^{+}$Absorption in the Vacuum Ultraviolet Spectrum of Molecular Nitrogen." In: The Journal of Chemical Physics 51.2 (1969), pp. 706716. DOI: 10.1063/1.1672060 (cit. on p. 31).

[28] Keith R. Lykke and Bruce D. Kay. "Two-photon spectroscopy of $\mathrm{N}_{2}$ : Multiphoton ionization, laser-induced fluorescence, and direct absorption via the $\mathrm{a}^{\prime}{ }^{1} \Sigma_{\mathrm{g}}^{+}$state." In: The Journal of Chemical Physics 95.4 (Aug. 1991), pp. 2252-2258. Dor: 10.1063/1. 460981 (cit. on p. 31).

[29] R. Schinke, J. A. Schmidt, and M. S. Johnson. "Photodissociation of $\mathrm{N}_{2} \mathrm{O}$ : Triplet states and triplet channel." In: Journal of Chemical Physics 135.19 (2011). Dor: 10.1063/1. 3660349 (cit. on pp. 31, 32).

[30] R. Schinke. "Photodissociation of $\mathrm{N}_{2} \mathrm{O}$ : Potential energy surfaces and absorption spectrum." In: Journal of Chemical Physics 134.6 (Feb. 2011), p. 064313. DOI: 10. 1063/1. 3553377 (cit. on p. 32).

[31] Darrel G. Hopper. " $A b$ initio multiple root optimization MCSCF study of the $\mathrm{C}_{\infty v} / \mathrm{C}_{s}$ excitation spectra and potential energy surfaces of $\mathrm{N}_{2} \mathrm{O}$." In: The Journal of Chemical Physics 80.9 (May 1984), pp. 4290-4316. DoI: 10. 1063/1. 447260 (cit. on p. 32). 
[32] J. D. Lambert. Vibrational and rotational relaxation in gases. Oxford: Clarendon Press, 1977, p. 141 (cit. on p. 34).

[33] H. Niehus and B. Krahl-Urban. "Improved technique in electron stimulated desorption ion angular distributions." In: Review of Scientific Instruments 52.1 (1981), pp. 56-59. DOI: 10 . 1063/1.1136446 (cit. on pp. 39, 40).

[34] Paul H. Kobrin, G. Alan Schick, James P. Baxter, and Nicholas Winograd. "Detector for measuring energy- and angle-resolved neutral-particle (EARN) distributions for material desorbed from bombarded surfaces." In: Review of Scientific Instruments 57.7 (1986), pp. 1354-1362. DOI: 10.1063/1.1138600 (cit. on p. 39).

[35] Dan J. Harding, Jannis Neugebohren, Daniel J. Auerbach, T. N. Kitsopoulos, and Alec M. Wodtke. "Using Ion Imaging to Measure Velocity Distributions in Surface Scattering Experiments." In: Journal of Physical Chemistry A 119.50 (2015), pp. 1225512262. DoI: 10.1021/acs . jpca. 5 b06272 (cit. on p. 43).

[36] Kenichi Tonokura and Toshinori Suzuki. "Slicing photofragment spatial distribution by laser sheet ionization." In: Chemical Physics Letters 224.1-2 (1994), pp. 1-6. DOI: 10.1016/0009 2614 ( 94 ) 00533 - 8 (cit. on p. 44).

[37] George Comsa and Rudolf David. "Dynamical parameters of desorbing molecules." In: Surface Science Reports 5.4 (Dec. 1985), pp. 145-198. DOI: 10 . 1016/0167 - 5729 ( 85 ) 90009 - 3 (cit. on pp. 45, 46, 50, 51, 131).

[38] C.T. Campbell, G. Ertl, H. Kuipers, and J. Segner. "A molecular beam investigation of the interactions of $\mathrm{CO}$ with a $\mathrm{Pt}(111)$ surface." In: Surface Science Letters 107.1 (May 1981), A222. DoI: 10.1016/0167-2584 (81) 90554- 5 (cit. on pp. 46, 62, 100).

[39] Otto Stern. "Nachtrag zu meiner Arbeit: Eine direkte Messung der thermischen Molekulargeschwindigkeit." In: Zeitschrift für Physik 3 (1920), pp. 417-421 (cit. on p. 50).

[40] Kenneth C. Janda, Jerry E. Hurst, Charles A. Becker, James P. Cowin, Daniel J. Auerbach, and Lennard Wharton. "Direct measurement of velocity distributions in argon beam-tungsten surface scattering." In: The Journal of Chemical Physics 72.4 (1980), p. 2403. DOI: 10.1063/1. 439434 (cit. on pp. 52, 80).

[41] Kai Golibrzuch, Pranav R. Shirhatti, Jan Geweke, Jörn Werdecker, Alexander Kandratsenka, Daniel J. Auerbach, Alec M. Wodtke, and Christof Bartels. "CO Desorption from a Catalytic Surface: Elucidation of the Role of Steps by Velocity-Selected Residence Time Measurements." In: Journal of the American Chemical Society 137.4 (2015), pp. 1465-1475. DOI: 10.1021 / ja509530k (cit. on pp. 61, 62, 70, 71, 90). 
[42] J. A. Serri, J. C. Tully, and M. J. Cardillo. "The influence of steps on the desorption kinetics of NO from Pt(111)." In: The Journal of Chemical Physics 79.3 (1983), pp. 1530-1540. DOI: 10. 1063/1. 445946 (cit. on pp. 67, 71).

[43] Laurens K. Verheij, Joachim Lux, A Brad Anton, Bene Poelsema, and George Comsa. "A molecular beam study of the interaction of CO molecules with a Pt (111) surface using pulse shape analysis." In: Surf. Sci. 182.3 (1987), pp. 390-410. DoI: 10.1016/0039-6028 (87)90008-2 (cit. on pp. 70, 71).

[44] G. E. Gdowski and R. J. Madix. "The effect of sulfur on CO adsorption/desorption on Pt(S)-[9(111) × (100)]." In: Surface Science 115.3 (Mar. 1982), pp. 524-540. DOI: 10 . $1016 / 0039$ 6028 (82) 90385 - 5 (cit. on pp. 70, 71).

[45] Dmitriy Borodin. "Probing Reactions at Surfaces using Ion Imaging - CO Oxidation at Atomically Flat and Stepped Surfaces of Platinum and Palladium." Master Thesis. Georg-AugustUniversity Goettingen, 2017 (cit. on pp. 71, 142).

[46] Jannis Neugebohren, Dmitriy Borodin, Hinrich W. Hahn, Jan Altschäffel, Alexander Kandratsenka, Daniel J. Auerbach, Charles T. Campbell, Dirk Schwarzer, Dan J. Harding, Alec M. Wodtke, and Theofanis N. Kitsopoulos. "Velocity-resolved kinetics of site-specific carbon monoxide oxidation on platinum surfaces." In: Nature 558.7709 (June 2018), pp. 280-283. DoI: 10 . 1038/ s41586-018-0188-x (cit. on pp. 79-82, 123, 130, 132, 134, 146, 147).

[47] E. Poehlmann, M. Schmitt, H. Hoinkes, and H. Wilsch. "Velocity distributions of carbondioxide molecules from the oxidation of CO on Pt(111)." In: Surface Science 287-288 (May 1993), pp. 269-272. DOI: 10 . 1016 / 0039 - 6028 ( 93 ) 90784 - H (cit. on pp. 80, 81, 129, 131).

[48] K.-H. Allers, H. Pfnür, P. Feulner, and D. Menzel. “Fast Reaction Products from the Oxidation of $\mathrm{CO}$ on $\mathrm{Pt}(111)$ : Angular and Velocity Distributions of the $\mathrm{CO}_{2}$ Product Molecules." In: The Journal of Chemical Physics 100.111 (1994), pp. 3985-3998. DOI: 10.1063/1. 466332 (cit. on pp. 80, 81, 129, 131, 133, 136).

[49] J. Segner, C. T. Campbell, G. Doyen, and G. Ertl. "Catalytic oxidation of $\mathrm{CO}$ on $\mathrm{Pt}(111)$ : The influence of surface defects and composition on the reaction dynamics." In: Surface Science 138.2-3 (Mar. 1984), pp. 505-523. DOI: 10. 1016/0039-6028 (84) 90262-0 (cit. on pp. 81, 88, 96, 129, 135-137).

[50] E. Poehlmann, M. Schmitt, H. Hoinkes, and H. Wilsch. "Bimodal angular and velocity distributions of $\mathrm{CO}_{2}$ desorbing after oxidation of CO on Pt (111)." In: Surface Review and Letters 
02.06 (Dec. 1995), pp. 741-758. DOI: 10.1142/S0218625X95000674 (cit. on pp. 81, 129, 133).

[51] Irving Langmuir. "The adsorption of gases on plane surfaces of glass, mica and platinum." In: Journal of the American Chemical Society 40.9 (1918), pp. 1361-1403. DOI: 10.1021/ja02242a004 (cit. on p. 87 ).

[52] Cyril Norman Hinshelwood. Kinetics of chemical change in gaseous systems. London: Clarendon Press, 1926 (cit. on p. 87).

[53] Charles A. Becker, James P. Cowin, Lennard Wharton, and Daniel J. Auerbach. " $\mathrm{CO}_{2}$ product velocity distributions for CO oxidation on platinum." In: The Journal of Chemical Physics 67.7 (1977), p. 3394. DoI: 10. 1063/1. 435289 (cit. on pp. 87, 131, 135).

[54] Robert L. Palmer and Joe N. Jr Smith. "Molecular beam study of CO oxidation on a (111) platinum surface." In: The Journal of Chemical Physics 60.4 (1974), p. 1453. Dor: 10.1063/1. 1681219 (cit. on pp. 87, 129, 135).

[55] C. T. Campbell, G. Ertl, H. Kuipers, and J. Segner. "A Molecular Beam Study of the Catalytic Oxidation of $\mathrm{CO}$ on a $\mathrm{Pt}(111)$ Surface." In: The Journal of Chemical Physics 73.111 (1980), pp. 58625873. DoI: 10.1063/1.440029 (cit. on pp. 87, 135, 136).

[56] Stefania Carmen Bobaru. "High-Pressure STM Studies of Oxidation Catalysis." Ph.D. Thesis. Leiden University, 2006, pp. 1118 (cit. on p. 88).

[57] J. E. Reutt-Robey, D. J. Doren, Y. J. Chabal, and S. B. Christman. "Microscopic CO Diffusion on a Pt(111) Surface by TimeResolved Infrared Spectroscopy." In: Physical Review Letters 61.24 (May 1988), pp. 2778-2781. DoI: 10 . 1103 / PhysRevLett . 61. 2778 (cit. on pp. 90, 139, 142, 143).

[58] T. H. Lin and G. A. Somorjai. "Modulated molecular beam scattering of $\mathrm{CO}$ and $\mathrm{NO}$ from $\mathrm{Pt}(111)$ and the stepped $\mathrm{Pt}(557)$ crystal surfaces." In: Surface Science 107.2-3 (1981), pp. 573-585. DOI: 10 . 1016/0039-6028 (81) 90546 - X (cit. on pp. 90, 137, 142, 143).

[59] Ryosuke Jinnouchi, Kensaku Kodama, and Yu Morimoto. “DFT calculations on $\mathrm{H}, \mathrm{OH}$ and $\mathrm{O}$ adsorbate formations on $\mathrm{Pt}(111)$ and $\mathrm{Pt}(332)$ electrodes." In: Journal of Electroanalytical Chemistry 716 (Mar. 2014), pp. 31-44. DoI: 10. 1016/j . j elechem. 2013 . 09. 031 (cit. on pp. 90, 112, 125, 143).

[6o] A. Nagoya, Ryosuke Jinnouchi, Kensaku Kodama, and Yu Morimoto. "DFT calculations on $\mathrm{H}, \mathrm{OH}$ and $\mathrm{O}$ adsorbate formations on Pt(322) electrode." In: Journal of Electroanalytical Chemistry 757 (Nov. 2015), pp. 116-127. DOI: 10.1016/ j . jelechem . 2015.09.009 (cit. on pp. 90, 112, 125, 137, 143). 
[61] C. T. Campbell, G. Ertl, H. Kuipers, and J. Segner. "A molecular beam study of the adsorption and desorption of oxygen from a Pt(111) surface." In: Surface Science 107.1 (May 1981), pp. 220-236. DOI: 10 . 1016/0039 - 6028 (81 ) 90622 - 1 (cit. on p. 97).

[62] R. Lewis and R. Gomer. "Adsorption of oxygen on platinum." In: Surface Science 12.2 (Oct. 1968), pp. 157-176. DoI: 10.1016/ 0039-6028 (68) 90121-0 (cit. on p. 97).

[63] J. D. Lambert. Numerical Methods for Ordinary Differential Systems: The Initial Value Problem. New York: Wiley, 1991 (cit. on p. 99).

[64] Adam C. Hindmarsh. "ODEPACK, A Systematized Collection of ODE Solvers." In: IMACS Transactions on Scientific Computation. Ed. by R. S. Stepleman. Vol. 1. Amsterdam: North-Holland, 1983, pp. 55-64 (cit. on p. 99).

[65] G. Ertl, M. Neumann, and K. M. Streit. "Chemisorption of CO on the Pt(111) surface." In: Surface Science 64.2 (1977), pp. 393410. DOI: 10. 1016/0039-6028 (77) 90052 - 8 (cit. on p. 100).

[66] H. Steininger, S. Lehwald, and H. Ibach. "On the adsorption of CO on Pt(111)." In: Surface Science 123.2-3 (1982), pp. 264282. DOI: 10.1016/0039-6028 (82) 90328- 4 (cit. on p. 100).

[67] John L. Gland and Edward B. Kollin. "Vibrational characterization of carbon monoxide oxidation on the $\mathrm{Pt}(111)$ surface." In: Surface Science 151.1 (1985), pp. 260-270. DOI: 10.1016/0039 6028 (85) 90465 - 0 (cit. on pp. 100, 136).

[68] J. Liu, M. Xu, and F. Zaera. "Determination of the rate limiting step in the oxidation of $\mathrm{CO}$ on $\mathrm{Pt}(111)$ surfaces." In: Catalysis Letters 37.1-2 (1996), pp. 9-13. DOI: 10.1007 / BF00813512 (cit. on pp. 100, 143).

[69] H. P. Bonzel and R. Ku. "On the kinetics of oxygen adsorption on a Pt(111) surface." In: Surface Science 40.1 (Oct. 1973), pp. 85101. DOI: 10.1016/0039-6028 (73) 90053-8 (cit. on p. 100).

[70] Cansin Badan, Rachael G. Farber, Yasmine Heyrich, Marc T. M. Koper, Daniel R. Killelea, and Ludo B. F. Juurlink. "Step-Type Selective Oxidation of Platinum Surfaces." In: The Journal of Physical Chemistry C 120.40 (Oct. 2016), pp. 22927-22935. DOI: 10.1021/acs. jpcc. 6 b05482 (cit. on p. 101).

[71] Bene Poelsema, Laurens K. Verheij, and George Comsa. "HeScattering Investigation of CO Migration on Pt(111)." In: Physical Review Letters 49.23 (Dec. 1982), pp. 1731-1735. Dor: 10 . 1103/PhysRevLett. 49.1731 (cit. on pp. 114, 139, 140). 
[72] Robert L Palmer. "Molecular beam study of oxygen and $C_{2}$ hydrocarbon chemisorption and reactions on $\mathrm{Pt}(111)$. ." In: Journal of Vacuum Science and Technology 12.6 (Nov. 1975), pp. 14031409. DOI: 10.1116/1.568551 (cit. on p. 129).

[73] Robert L. Palmer and Joe N. Smith. "Recent Studies of Chemical Interactions on Surfaces Using Molecular Beams." In: Catalysis Reviews 12.1 (1975), pp. 279-301. DOI: 10/ cbps2k (cit. on p. 129).

[74] P.R. Norton. "Surface analysis of platinum by x-ray photoelectron spectroscopy (XPS)." In: Surface Science 44.2 (Aug. 1974), pp. 624-628. DOI: 10 . 1016/0039-6028 (74 ) 90142 - 3 (cit. on p. 131).

[75] H. Davy. "On the Fire-Damp of Coal Mines, and on Methods of Lighting the Mines So as to Prevent Its Explosion." In: Philosophical Transactions of the Royal Society of London 106.January (1816), pp. 1-22. DoI: 10.1098/rstl . 1816.0001 (cit. on p. 135$)$.

[76] W. Henry. "XLIV. On the action of finely-divided platinum on gaseous mixtures, and its application to their analysis." In: Philosophical Magazine Series 1 65.May (1825), pp. 269-283. DoI: 10. 1080/14786442508628435 (cit. on p. 135).

[77] Irving Langmuir. "Chemical reactions at very low pressures. I. The clean-up in a tungsten lamp." In: Journal of the American Chemical Society 35.2 (Feb. 1913), pp. 105-127. DoI: 10.1021/ ja02191a001 (cit. on p. 135).

[78] Irving Langmuir. "Chemical reactions at very low pressures. II. The chemical clean-up of nitrogen in a tungsten lamp." In: Journal of the American Chemical Society 1310 (1913). DoI: 10 . 1021/ja02197a002 (cit. on p. 135).

[79] Irving Langmuir. "Surface Chemistry." In: Chemical Reviews 13.2 (Oct. 1933), pp. 147-191. DOI: 10.1021/ cr60045a001 (cit. on p. 135).

[8o] Irving Langmuir. "The mechanism of the catalytic action of platinum in the reactions $2 \mathrm{CO}+\mathrm{O}_{2}=2 \mathrm{CO}_{2}$ and $2 \mathrm{H}_{2}+\mathrm{O}_{2}=$ $2 \mathrm{H}_{2} \mathrm{O}$." In: Transactions of the Faraday Society 17.1905 (1922), p. 621. DOI: 10.1039/tf9221700621 (cit. on p. 135).

[81] F. L. Hughes and Henry Levinstein. "Mean Adsorption Lifetime of $\mathrm{Rb}$ on Etched Tungsten Single Crystals: Neutrals." In: Physical Review 113.4 (Feb. 1959), pp. 1036-1038. Dor: 10.1103/ PhysRev. 113.1036 (cit. on p. 135).

[82] T. Engel and G. Ertl. "Elementary Steps in the Catalytic Oxidation of Carbon Monoxide on Platinum Metals." In: Advances in Catalysis. Vol. 28. 1979, pp. 1-78. DOI: 10.1016/S0360- 0564 ( 08 ) 60133- 9 (cit. on p. 135). 
[83] H. P. Bonzel and R. Ku. "Carbon Monoxide Oxidation on a $\operatorname{Pt}(110)$ Single Crystal Surface." In: Journal of Vacuum Science and Technology 9.2 (Mar. 1972), pp. 663-667. DoI: 10.1116/1. 1317748 (cit. on p. 135).

[84] H. P. Bonzel and R. Ku. "Mechanisms of the catalytic carbon monoxide oxidation on Pt (110)." In: Surface Science 33.1 (Oct. 1972), pp. 91-106. DOI: 10 . 1016/0039 - 6028 (72) 90101 - X (cit. on p. 135).

[85] G Ertl. "Heterogeneous catalysis on the atomic scale." In: Chemical record (New York, N.Y.) 1.1 (2001), pp. 33-45. DOI: 10.1016/ S1381- 1169 (01) 00460- 5 (cit. on p. 135).

[86] John L. Gland and Edward B. Kollin. "Carbon monoxide oxidation on the $\operatorname{Pt}(111)$ surface: Temperature programmed reaction of coadsorbed atomic oxygen and carbon monoxide." In: J. Chem. Phys. 78.2 (1983), p. 963. DoI: 10.1063/1. 444801 (cit. on p. 136).

[87] Sohail Akhter and J. M. White. "The effect of oxygen islanding on $\mathrm{CO}$ and $\mathrm{H}_{2}$ oxidation on Pt(111)." In: Surface Science 171.3 (1986), pp. 527-542. DOI: 10.1016/0039-6028 (86) 91058 - 7 (cit. on pp. 136, 144).

[88] J. Wintterlin, S. Völkening, T. V. W. Janssens, T. Zambelli, and G. Ertl. "Atomic and Macroscopic Reaction Rates of a SurfaceCatalyzed Reaction." In: Science 278.5345 (Dec. 1997), pp. 19311934. DOI: 10.1126/science. 278. 5345.1931 (cit. on pp. 136, 142, 143).

[89] Jiazhan $\mathrm{Xu}$ and John T. Yates. "Catalytic oxidation of $\mathrm{CO}$ on Pt(335): A study of the active site." In: The Journal of Chemical Physics 99.1 (July 1993), pp. 725-732. DoI: 10.1063/1.465745 (cit. on p. 137).

[9o] Thomas Engel and Philip Reid. Physikalische Chemie. 1st ed. München: Pearson Education, 2006, pp. 1024-1025 (cit. on p. 138).

[91] Paul L. Houston. Chemical Kinetics and Reaction Dynamics. Mineola, New York: Dover Publications, Inc., 2001, pp. 183-185 (cit. on p. 138).

[92] V. J. Kwasniewski and L. D. Schmidt. "Surface diffusion of CO on Pt(111)." In: Surface Science 274.3 (Aug. 1992), pp. 329-340. DOI: 10.1016/0039-6028 (92) 90838-W (cit. on p. 139).

[93] C. M. Yim, K. L. Man, Xudong Xiao, and M. S. Altman. "Lowenergy electron microscopy of $\mathrm{CO} / \mathrm{Pt}$ (111) surface diffusion by nonequilibrium coverage profile evolution." In: Physical Review $B$ - Condensed Matter and Materials Physics 78.15 (2008), pp. 1-9. DOI: 10.1103/PhysRevB. 78.155439 (cit. on p. 139). 
[94] J. E. Reutt-Robey, D. J. Doren, Y. J. Chabal, and S. B. Christman. "CO diffusion on $\mathrm{Pt}(111)$ with time-resolved infrared-pulsed molecular beam methods: Critical tests and analysis." In: The Journal of Chemical Physics 93.12 (Dec. 1990), pp. 9113-9129. Dor: 10.1063/1. 459202 (cit. on pp. 139, 140).

[95] Jianwei Ma, Xudong Xiao, N. J. DiNardo, and M. M. T. Loy. "Diffusion of CO on $\mathrm{Pt}(111)$ studied by an optical diffraction method." In: Physical Review B 58.8 (1998), pp. 4977-4983. Dor: 10.1103/PhysRevB. 58.4977 (cit. on pp. 139, 140).

[96] Kedong Wang, Fangfei Ming, Qiang Huang, Xieqiu Zhang, and Xudong Xiao. "Study of CO diffusion on stepped Pt(111) surface by scanning tunneling microscopy." In: Surface Science 604.3-4 (2010), pp. 322-326. DOI: 10 . 1016/j . susc . 2009 . 11.024 (cit. on pp. 139, 140).

[97] Radek Erban, Jonathan Chapman, and Philip Maini. A practical guide to stochastic simulations of reaction-diffusion processes. 2007 (cit. on p. 141).

[98] John C. Polanyi. "Some Concepts in Reaction Dynamics." In: Science 236 (1987), pp. 680-690. Dor: 10 . 1126 / science . 236. 4802.680 (cit. on p. 141).

[99] Ronald P. Bell. "The Theory of Reactions Involving Proton Transfers." In: Proceedings of the Royal Society A: Mathematical, Physical and Engineering Sciences 154.882 (Apr. 1936), pp. 414429. DOI: 10.1098/rspa. 1936.0060 (cit. on p. 141).

[10o] M. G. Evans and M. Polanyi. "Inertia and driving force of chemical reactions." In: Transactions of the Faraday Society 34 (1938), p. 11. DOI: 10.1039/tf9383400011 (cit. on p. 141).

[101] George S. Hammond. "A Correlation of Reaction Rates." In: Journal of the American Chemical Society 77.2 (1955), pp. 334-338. DOI: 10.1021/ja01607a027 (cit. on p. 141).

[102] Andreas Eichler. "CO oxidation on transition metal surfaces: reaction rates from first principles." In: Surface Science 498.3 (Feb. 2002), pp. 314-320. DOI: 10. 1016/S0039-6028 (01) 018052 (cit. on pp. 142, 149).

[103] András Szabó, Michael A. Henderson, and John T. Yates. “Oxidation of $\mathrm{CO}$ by oxygen on a stepped platinum surface: Identification of the reaction site." In: The Journal of Chemical Physics 96.8 (1992), p. 6191. DOI: 10.1063/1.462636 (cit. on p. 142).

[104] Masanari Nagasaka, Hiroshi Kondoh, Ikuyo Nakai, and Toshiaki Ohta. "Oxygen island formation on $\mathrm{Pt}(111)$ studied by dynamic Monte Carlo simulation." In: The Journal of Chemical Physics 122.4 (Jan. 2005), p. 044715. DOI: 10.1063/1. 1835270 (cit. on p. 144). 
[105] Hairong Tang, Anton Van der Ven, and Bernhardt L. Trout. "Phase diagram of oxygen adsorbed on platinum (111) by firstprinciples investigation." In: Physical Review B 70.4 (July 2004), p. 045420. Dor: 10.1103/PhysRevB. 70.045420 (cit. on p. 144).

[106] Joost Wintterlin. Private Communications. 2017 (cit. on p. 144).

[107] S. Völkening and J. Wintterlin. "CO oxidation on Pt(111) Scanning tunneling microscopy experiments and Monte Carlo simulations." In: The Journal of Chemical Physics 114.14 (Apr. 2001), pp. 6382-6395. DOI: 10.1063/1.1343836 (cit. on p. 144).

[108] Jens K. Nørskov, Thomas Bligaard, Britt Hvolbæk, Frank AbildPedersen, Ib Chorkendorff, and Claus H. Christensen. "The nature of the active site in heterogeneous metal catalysis." In: Chemical Society Reviews 37.10 (2008), p. 2163. DOI: 10.1039 / b800260f (cit. on p. 147).

[109] Henry Eyring. "The Activated Complex in Chemical Reactions." In: The Journal of Chemical Physics 3.2 (1935), pp. 107-115. DOI: 10.1063/1. 1749604 (cit. on pp. 148, 149).

[110] C. Díaz, R. A. Olsen, H. F. Busnengo, and G. J. Kroes. “Dynamics on six-dimensional potential energy surfaces for $\mathrm{H}_{2} / \mathrm{Cu}(111)$ : Corrugation reducing procedure versus modified shepard interpolation method and PW91 versus RPBE." In: Journal of Physical Chemistry C 114.25 (2010), pp. 11192-11201. DOI: 10. 1021/jp1027096 (cit. on pp. 149, 150).

[111] Mikkel Jørgensen and Henrik Grönbeck. "Adsorbate Entropies with Complete Potential Energy Sampling in Microkinetic Modeling." In: Journal of Physical Chemistry C 121.13 (2017), pp. 71997207. DOI: 10.1021/acs. jpcc.6b11487 (cit. on p. 149). 

Part IV

APPENDIX 

APPENDIX: KINETIC MODEL RESULT PLOTS

A.I PT(111) RESULTS

The result of fitting the numeric solution to the experimental kinetic traces is shown in figure A.I on the next pages. The hyperthermal channel's kinetic trace is plotted in red (experimental data as open circles, numeric solution as a curve) and the thermal channel's kinetic trace is plotted in blue. The ts- and ss-reaction contribution to the thermal channel are plotted as black and green dashed curves. 


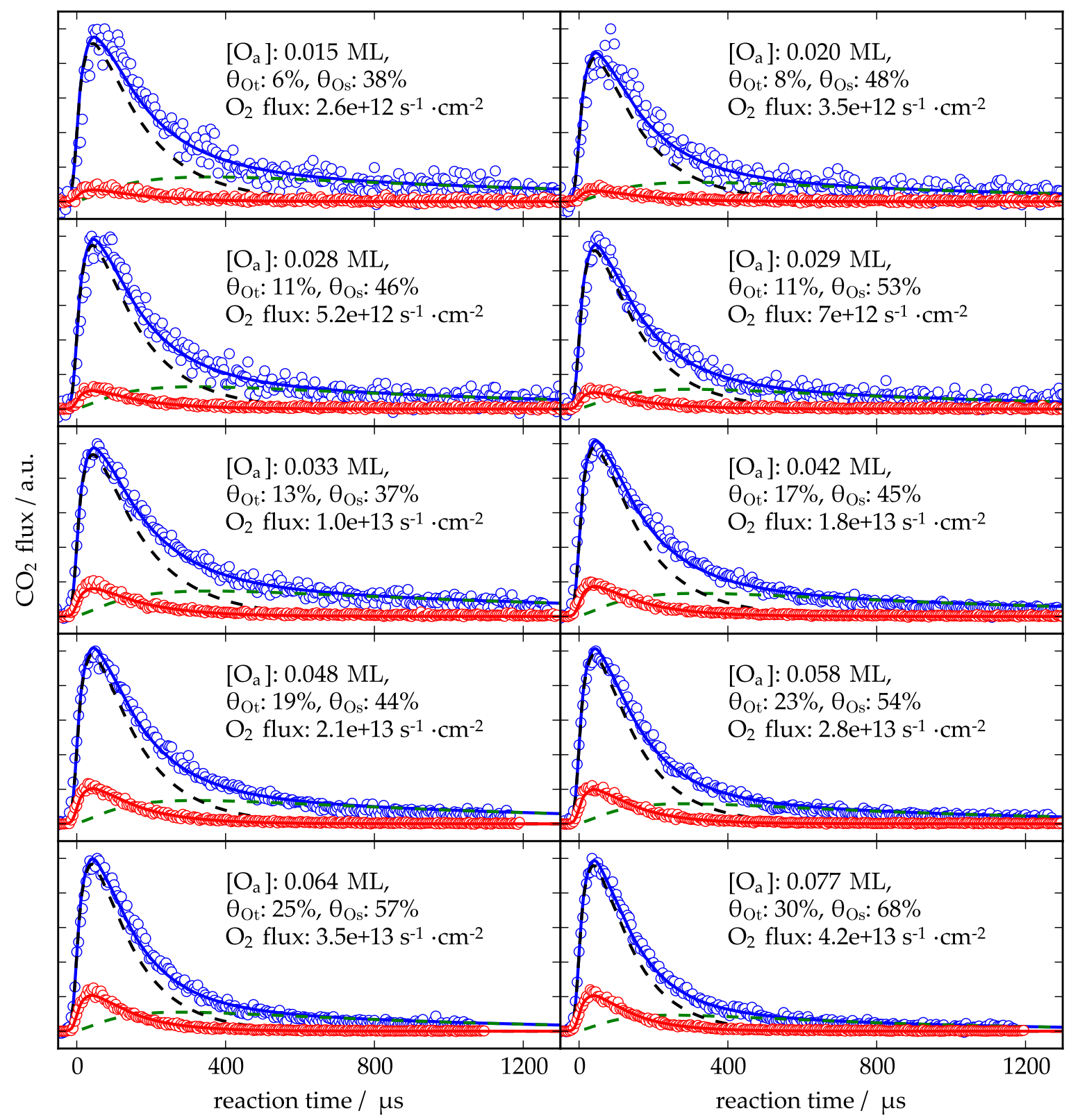

(a) $290^{\circ} \mathrm{C}$, RRRs: $0.25,0.33,0.5,0.67,1,1.67,2,2.66,3.33,4$ 


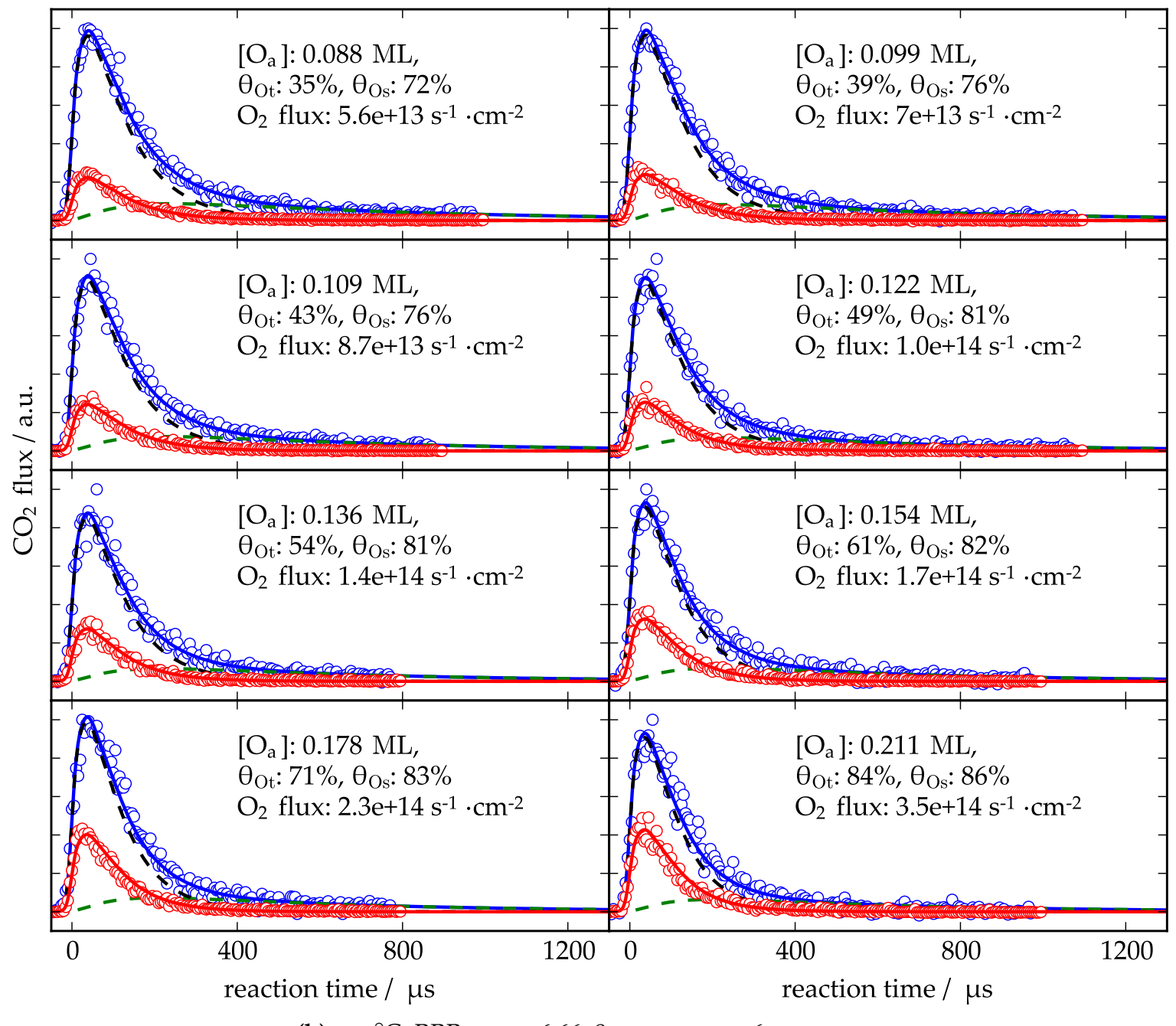

(b) $290^{\circ} \mathrm{C}$, RRRs: $5.29,6.66,8.33,10,13.3,16.7,22.2,33.3$

Figure A.1: Kinetic traces on $\operatorname{Pt}(111)$. The thermal channel is plotted in blue, the hyperthermal channel in red. The contributions to the thermal channel, the ts- and the ss-reactions, are plotted as black and green dashed curves, respectively. 


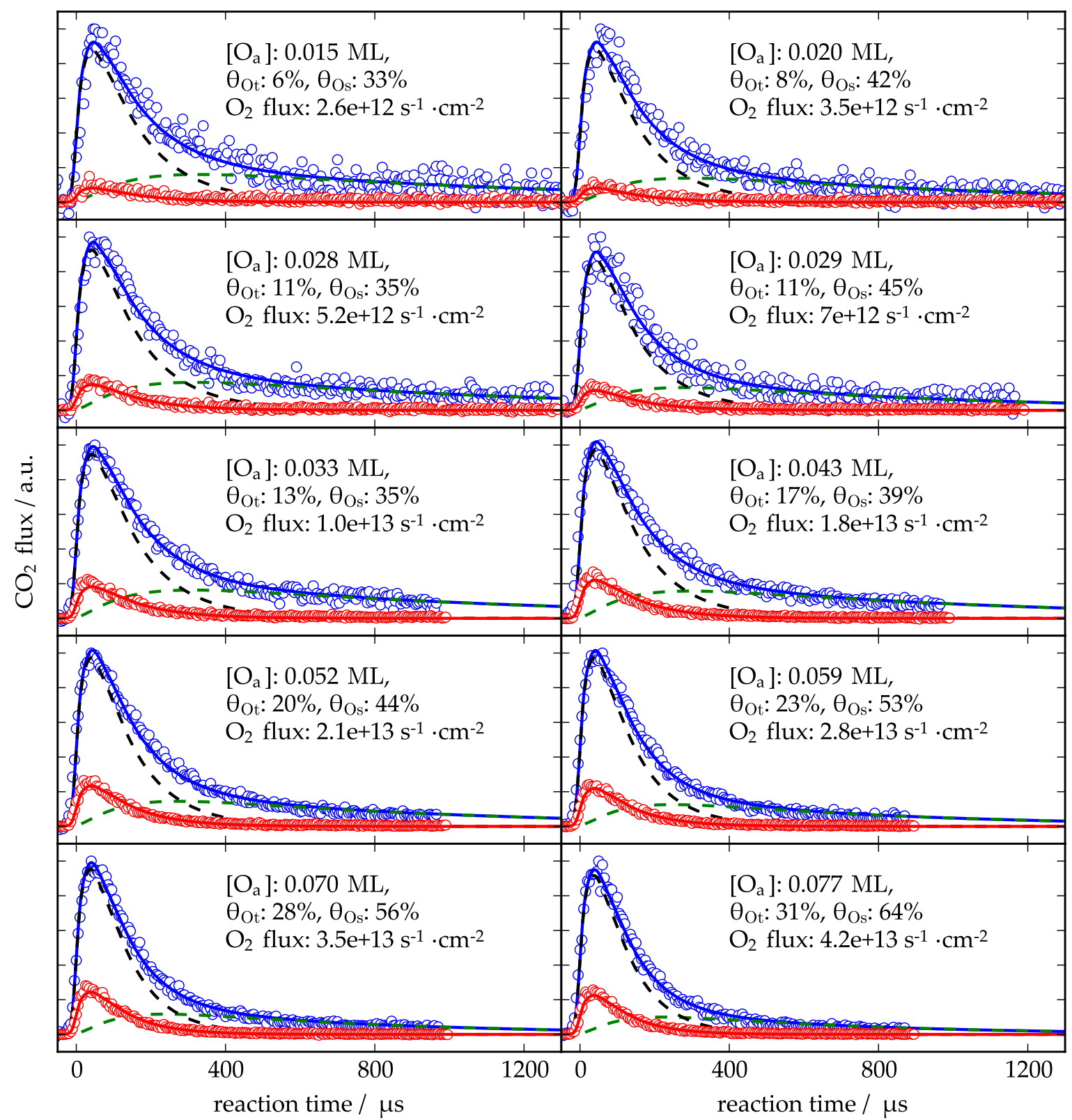

(c) $300{ }^{\circ} \mathrm{C}$, RRRs: $0.25,0.33,0.5,0.67,1,1.67,2,2.66,3.33,4$ 


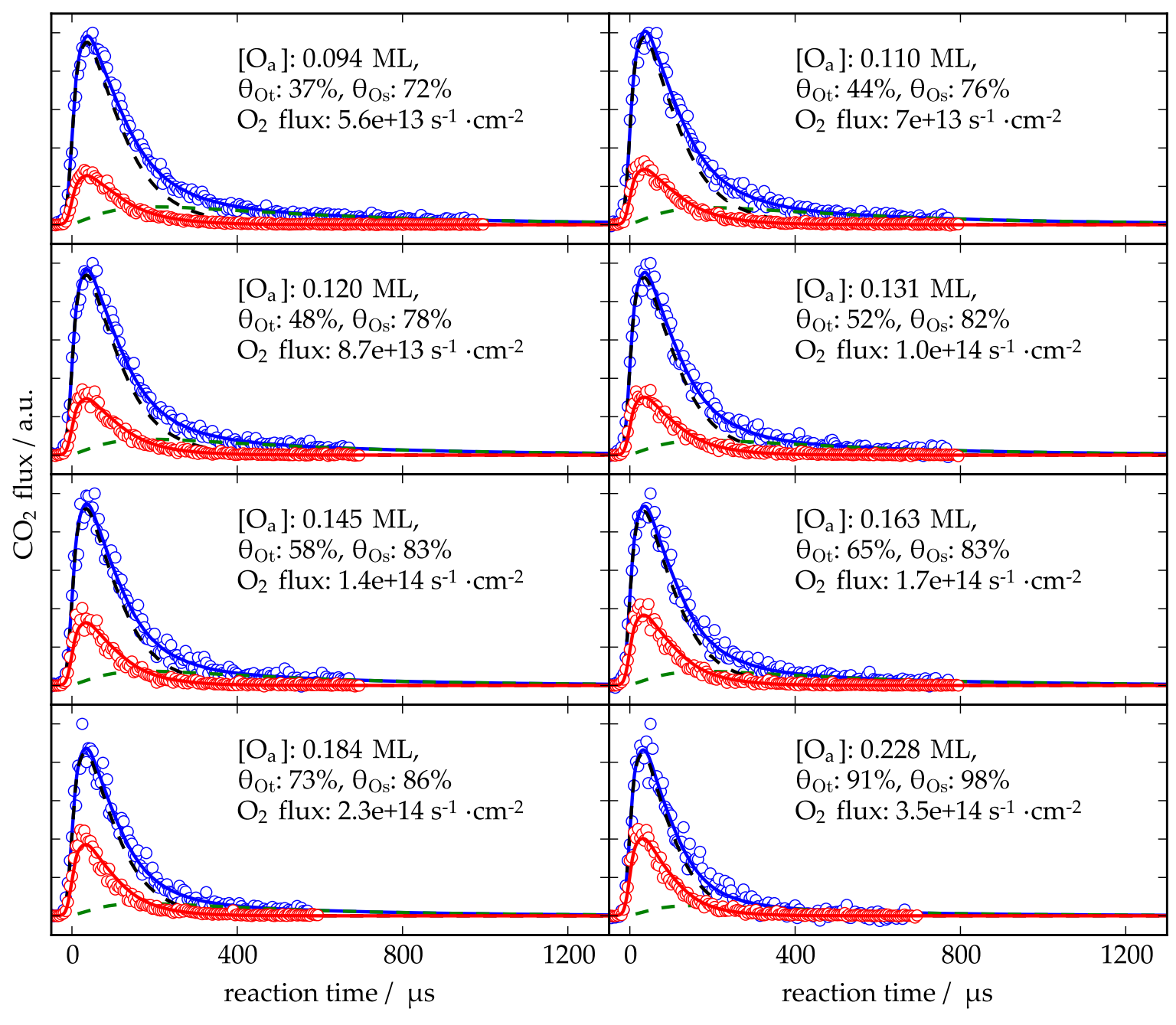

(d) $300{ }^{\circ} \mathrm{C}$, RRRs: $5.29,6.66,8.33,10,13.3,16.7,22.2,33.3$

Figure A.1: Kinetic traces on $\operatorname{Pt}(111)$ (continued). 


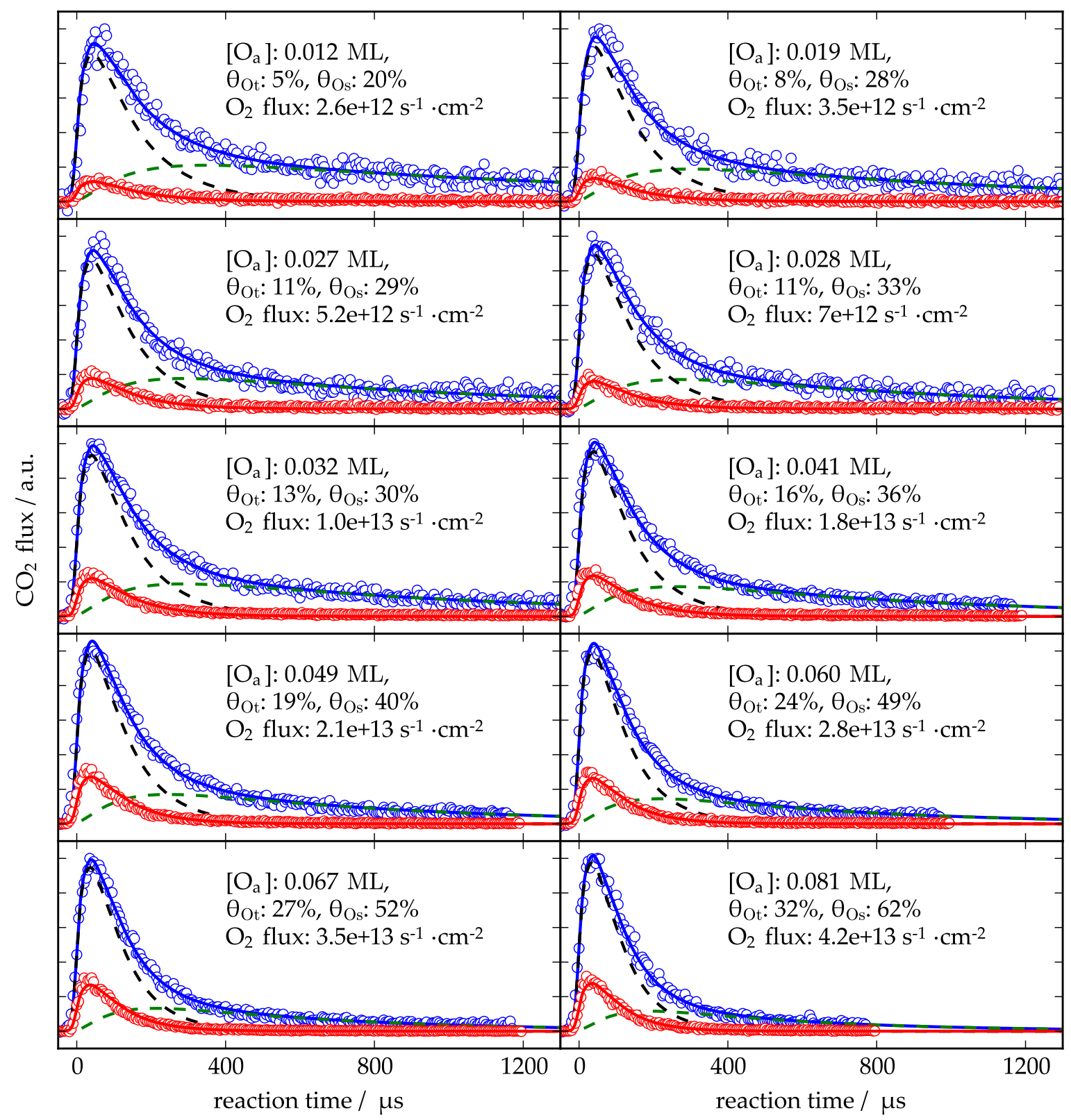

(e) $310^{\circ} \mathrm{C}$, RRRs: $0.25,0.33,0.5,0.67,1,1.67,2,2.66,3.33,4$ 


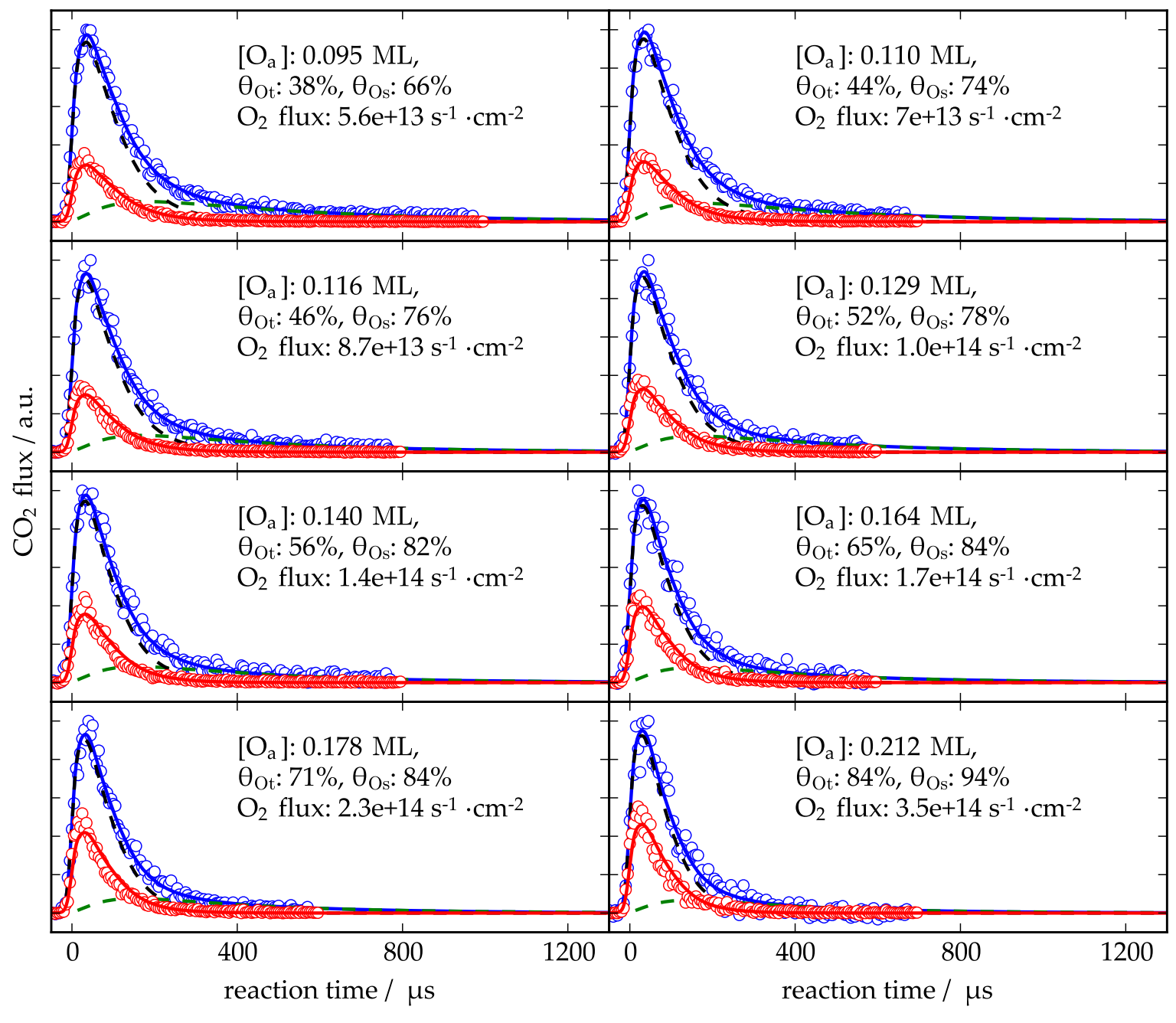

(f) $310^{\circ} \mathrm{C}$, RRRs: $5.29,6.66,8.33,10,13.3,16.7,22.2,33.3$

Figure A.1: Kinetic traces on $\operatorname{Pt}(111)$ (continued). 


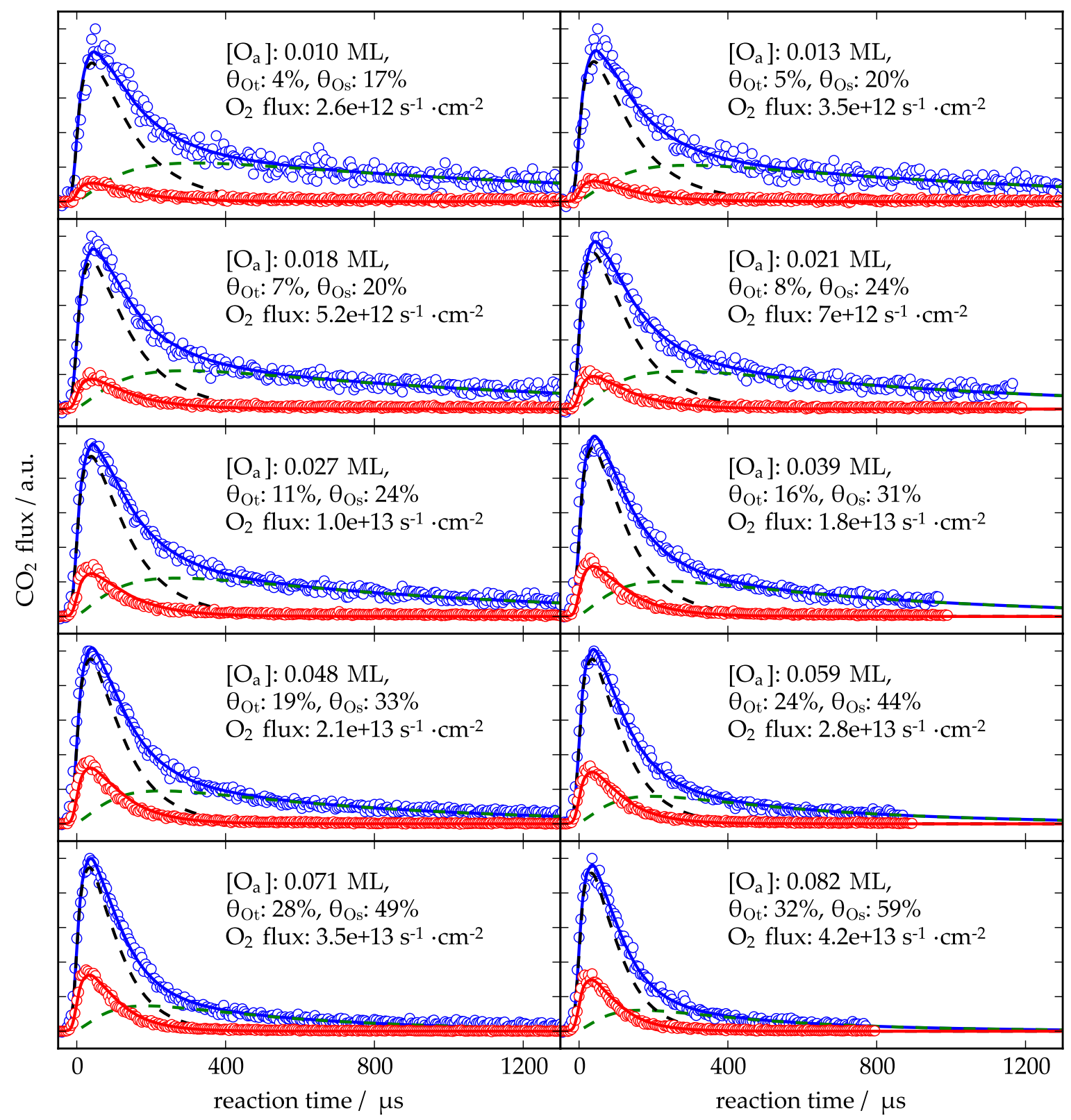

(g) $320^{\circ} \mathrm{C}$, RRRs: $0.25,0.33,0.5,0.67,1,1.67,2,2.66,3.33,4$ 


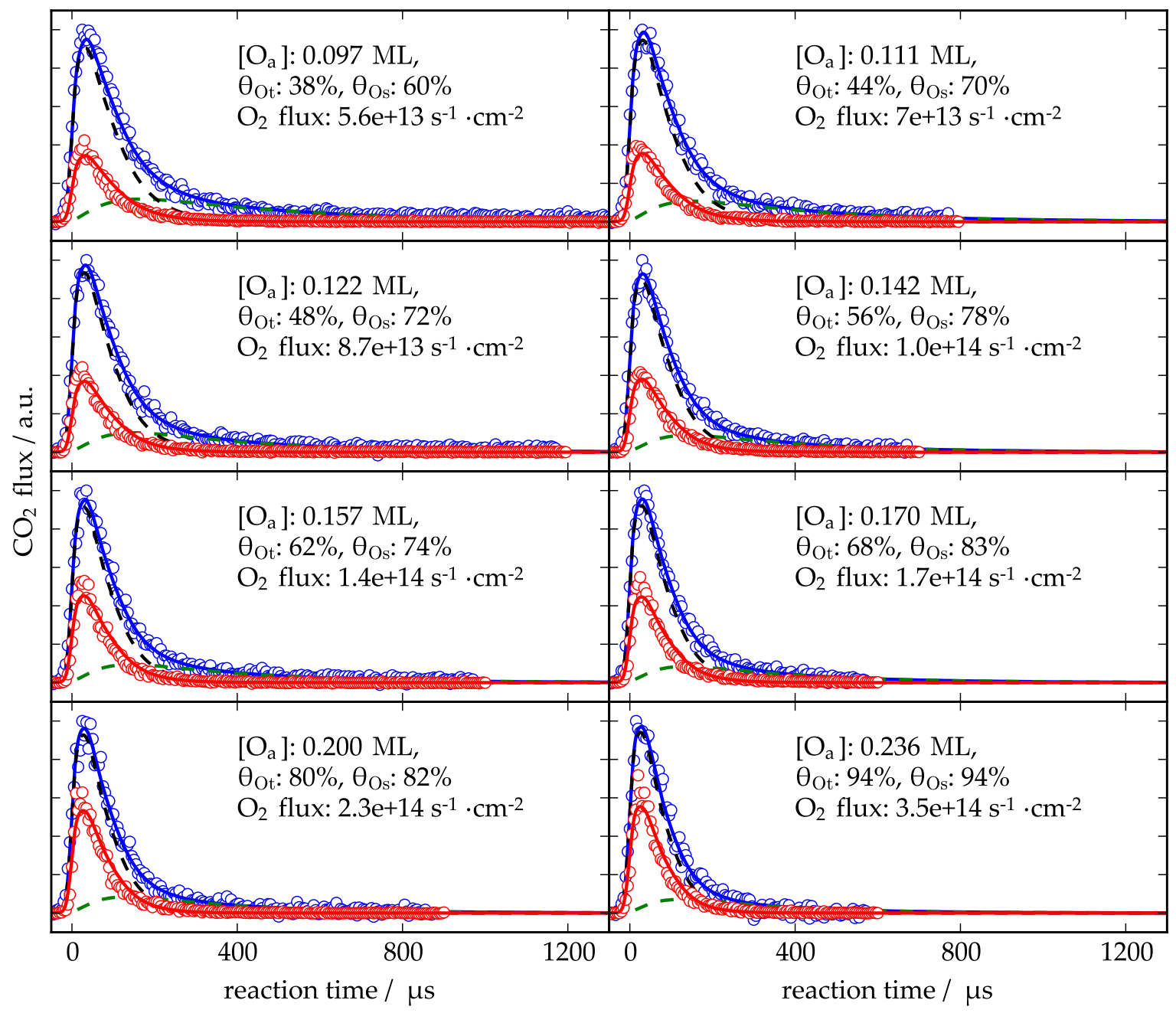

(h) $320^{\circ} \mathrm{C}$, RRRs: $5.29,6.66,8.33,10,13.3,16.7,22.2,33.3$

Figure A.1: Kinetic traces on $\operatorname{Pt}(111)$ (continued). 


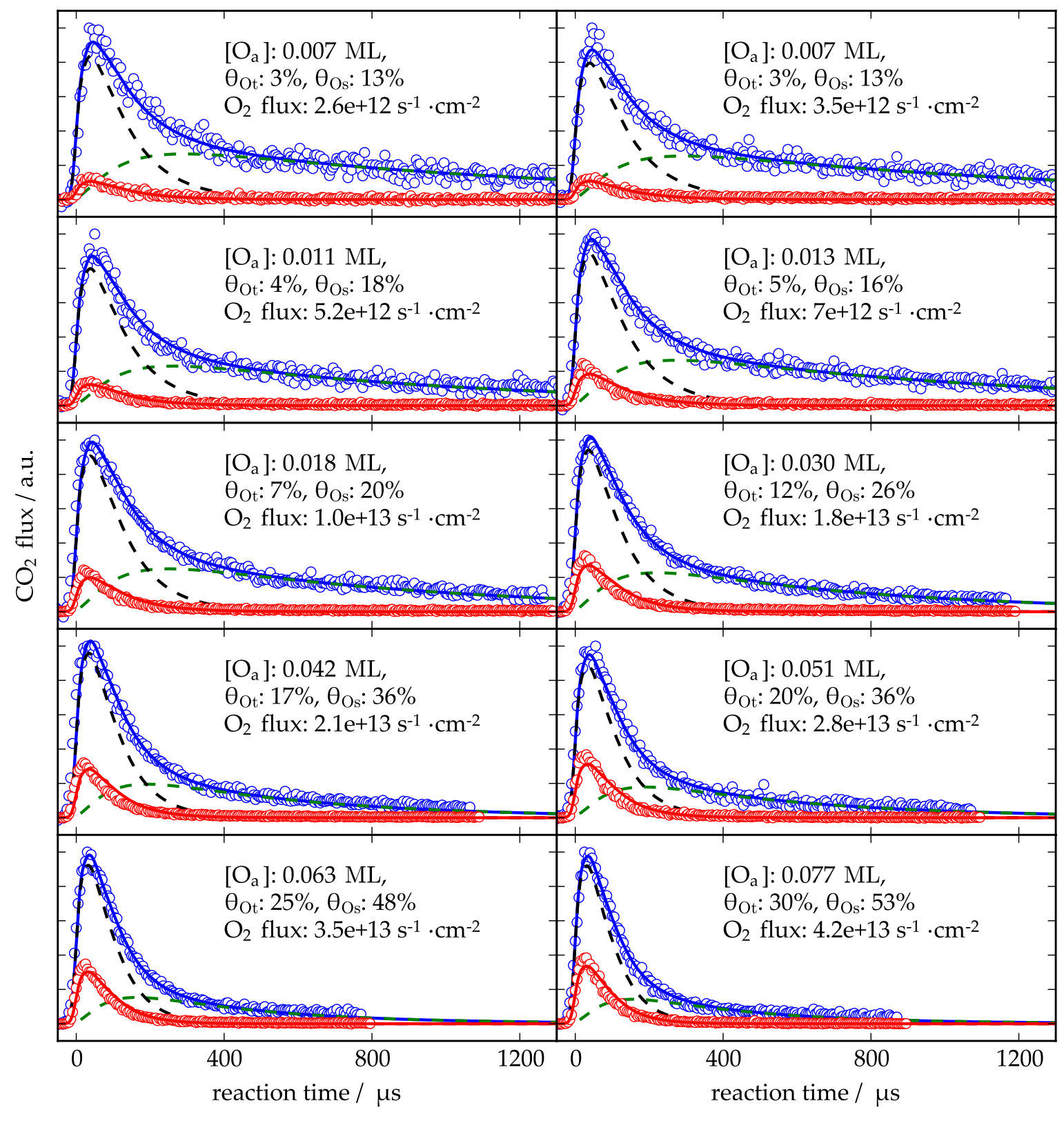

(i) $330^{\circ} \mathrm{C}$, RRRs: $0.25,0.33,0.5,0.67,1,1.67,2,2.66,3.33,4$ 


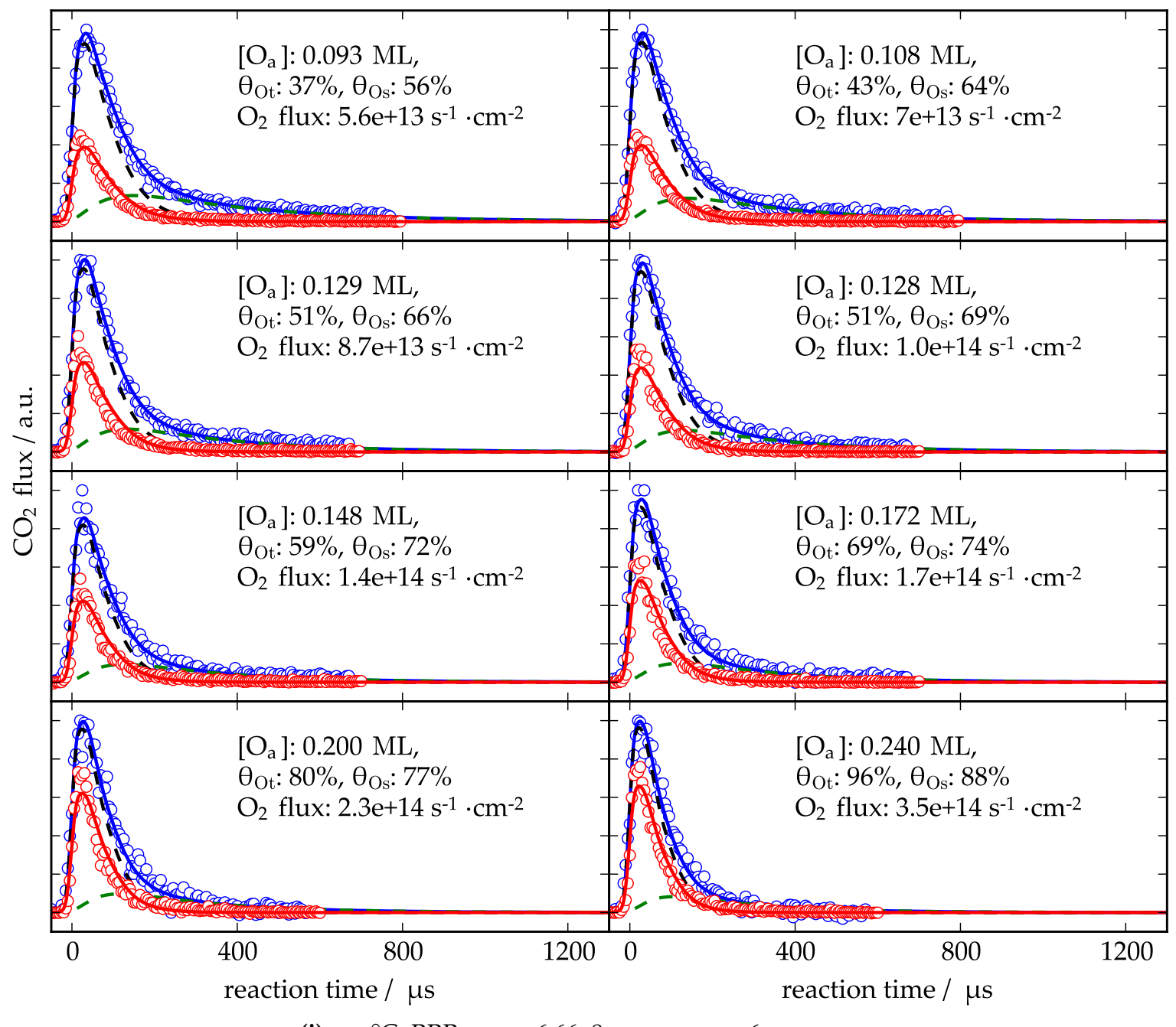

(j) $330^{\circ} \mathrm{C}$, RRRs: $5.29,6.66,8.33,10,13 \cdot 3,16.7,22.2,33.3$

Figure A.1: Kinetic traces on $\operatorname{Pt}(111)$ (continued). 


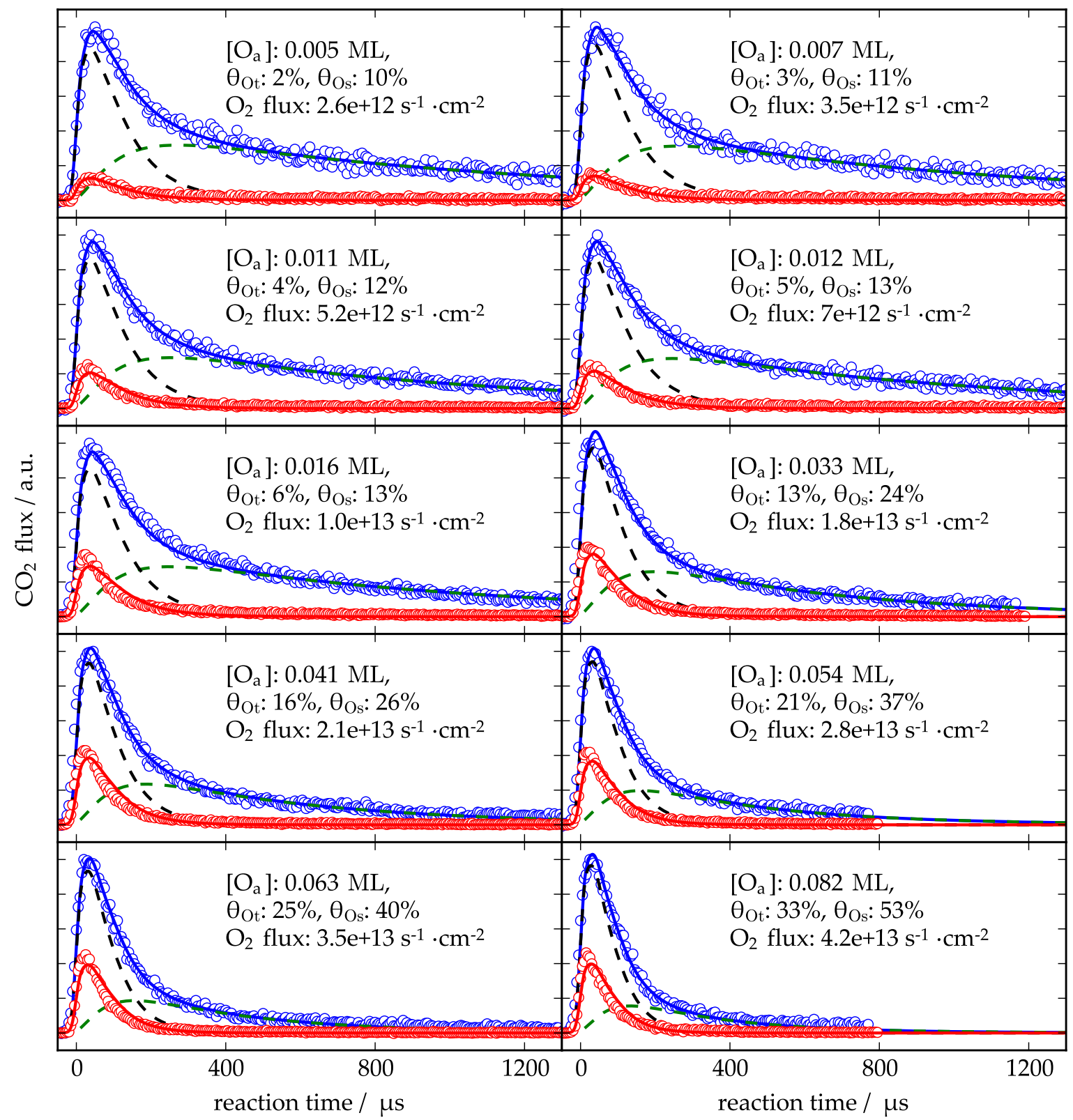

(k) $340^{\circ} \mathrm{C}$, RRRs: $0.25,0.33,0.5,0.67,1,1.67,2,2.66,3.33,4$ 


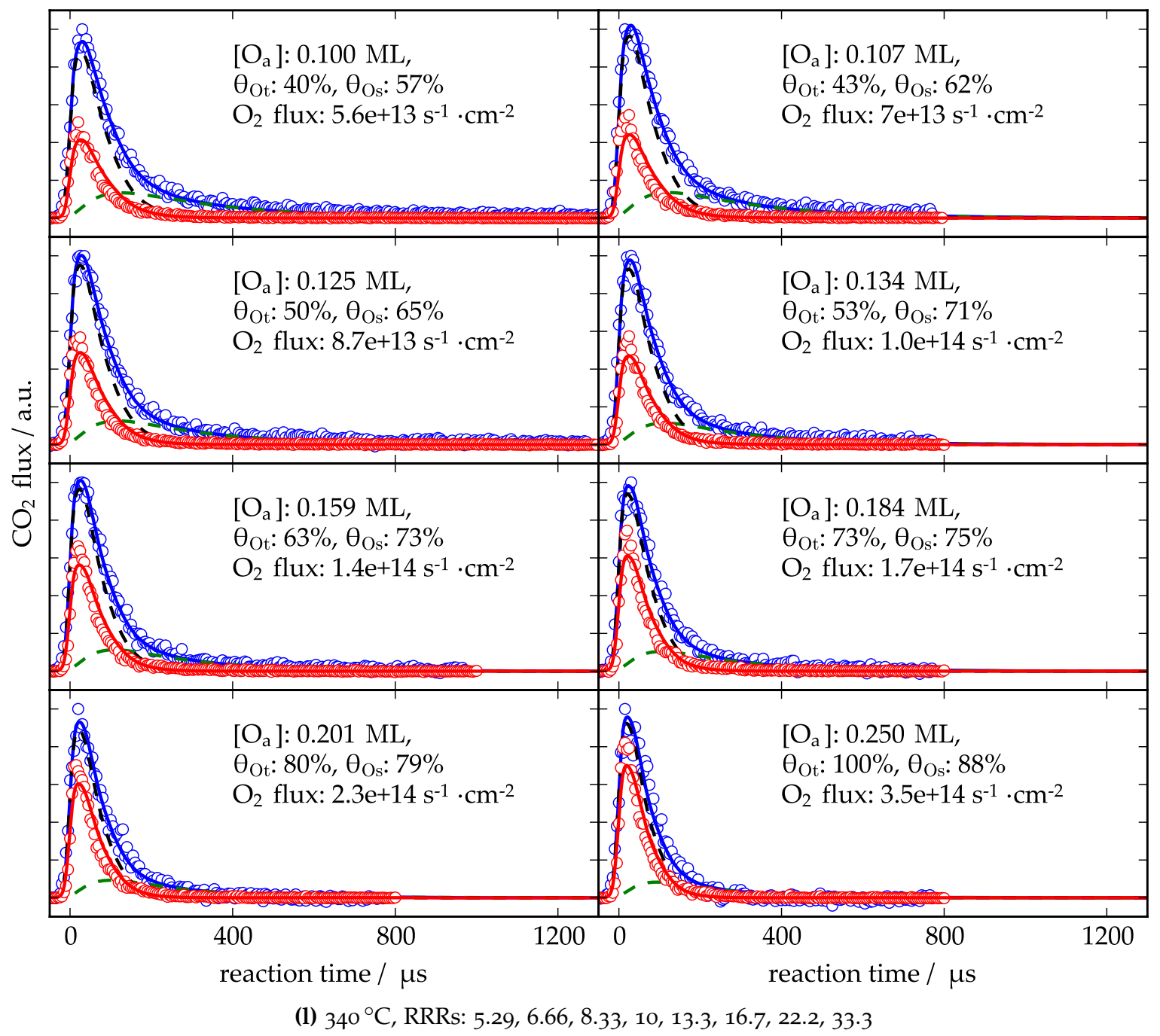

Figure A.1: Kinetic traces on $\operatorname{Pt}(111)$ (continued). 


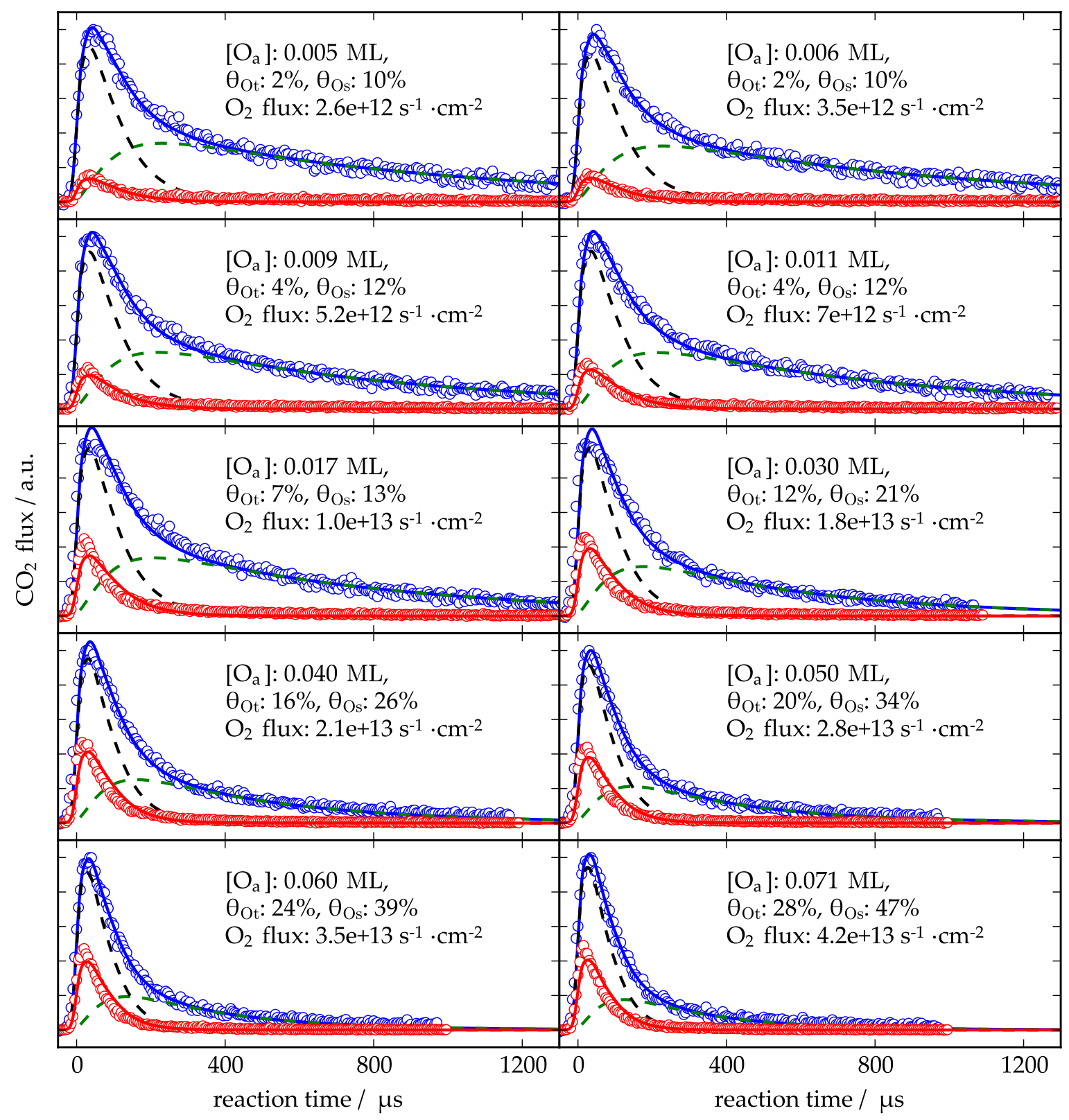

(m) $350^{\circ} \mathrm{C}$, RRRs: $0.25,0.33,0.5,0.67,1,1.67,2,2.66,3.33,4$ 


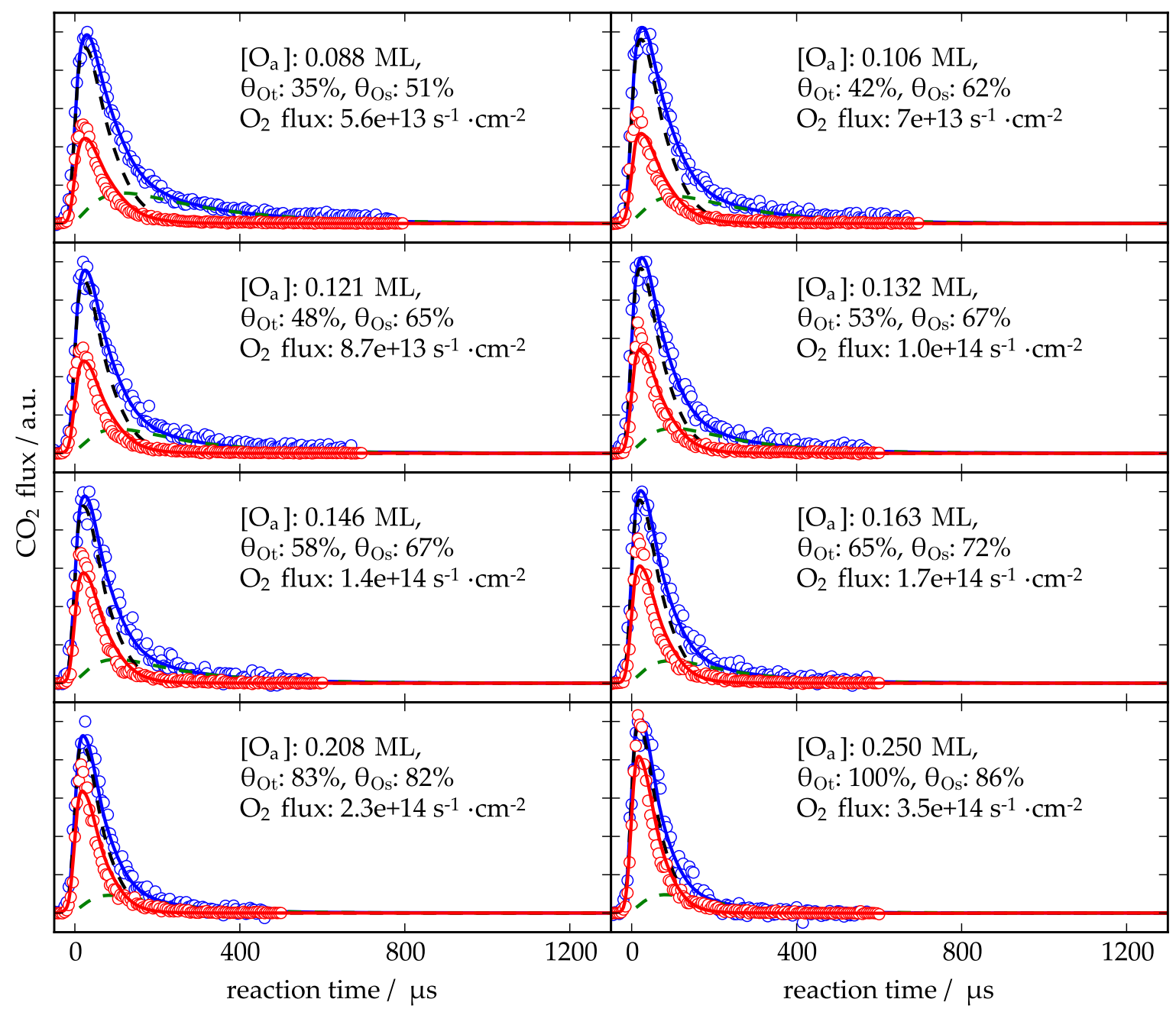

(n) $350^{\circ} \mathrm{C}$, RRRs: $5.29,6.66,8.33,10,13.3,16.7,22.2,33.3$

Figure A.1: Kinetic traces on $\operatorname{Pt}(111)$ (continued). 


\section{A.2 PT(332) RESULTS}

The results on $\mathrm{Pt}$ (332) are presented in the same way in figure A.2 on the next pages. The hyperthermal channel kinetic is plotted in red (experimental data as open circles, numeric solution as a curve) and the thermal channel is plotted in blue. Open circles were used to denote that the flux was corrected to represent the relative flux integrated over all angles and speeds. The ts- and ss-reactions contribution to the thermal channel are plotted as black and green dashed curves, respectively. 


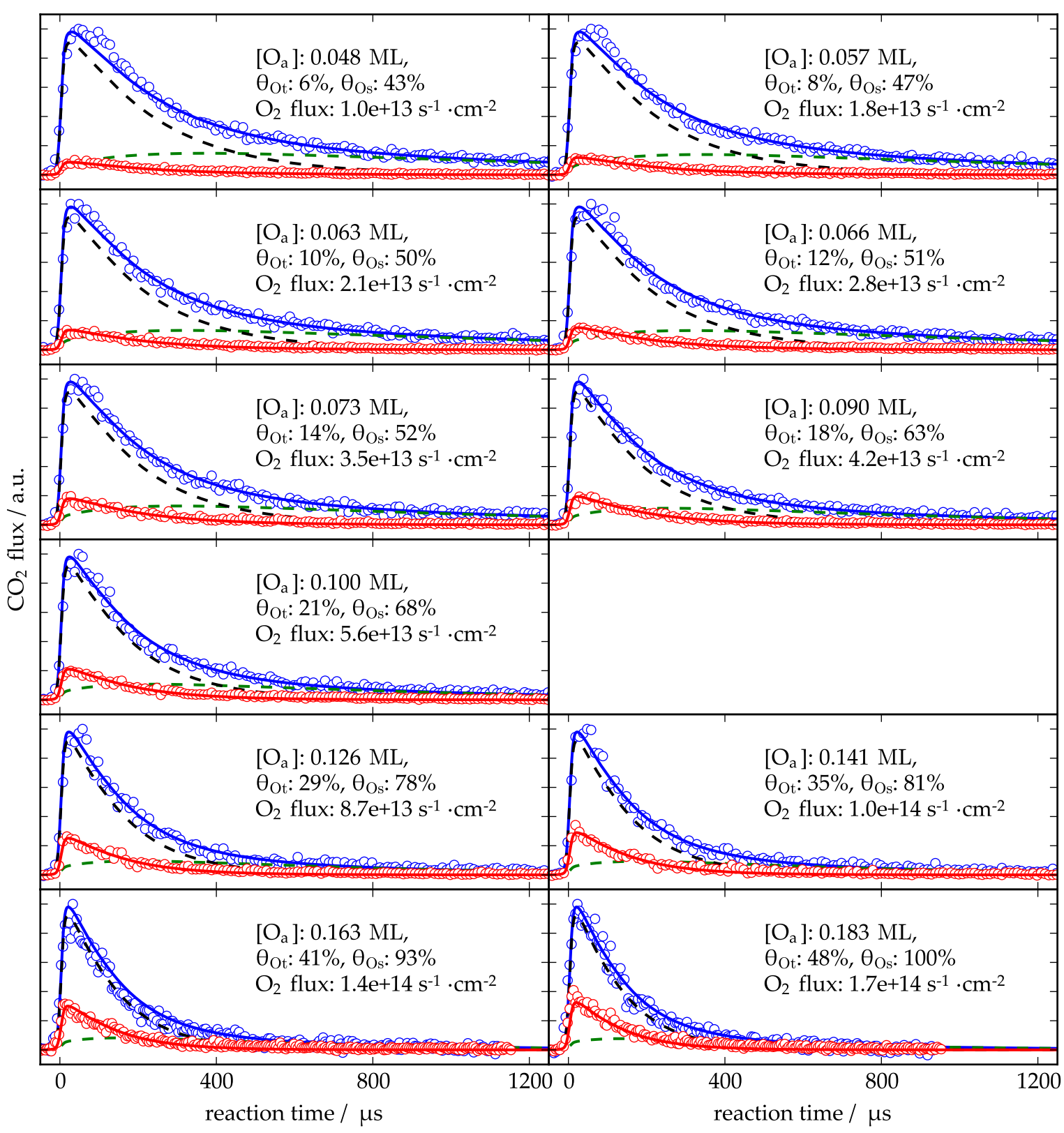

(a) $290{ }^{\circ} \mathrm{C}$, RRRs: $1,1.67,2,2.66,3.33,4,5.29,8.33,10,13.3,16.7$

Figure A.2: Kinetic traces on $\operatorname{Pt}(332)$. The thermal channel is plotted in blue and the hyperthermal channel is plotted in red. The contributions to the thermal channel, the ts- and the ss-reactions, are plotted as black and green dashed curves, respectively. The scan at an RRR of 6.66 at $290^{\circ} \mathrm{C}$ is missing, the panel was intentionally left blank. 


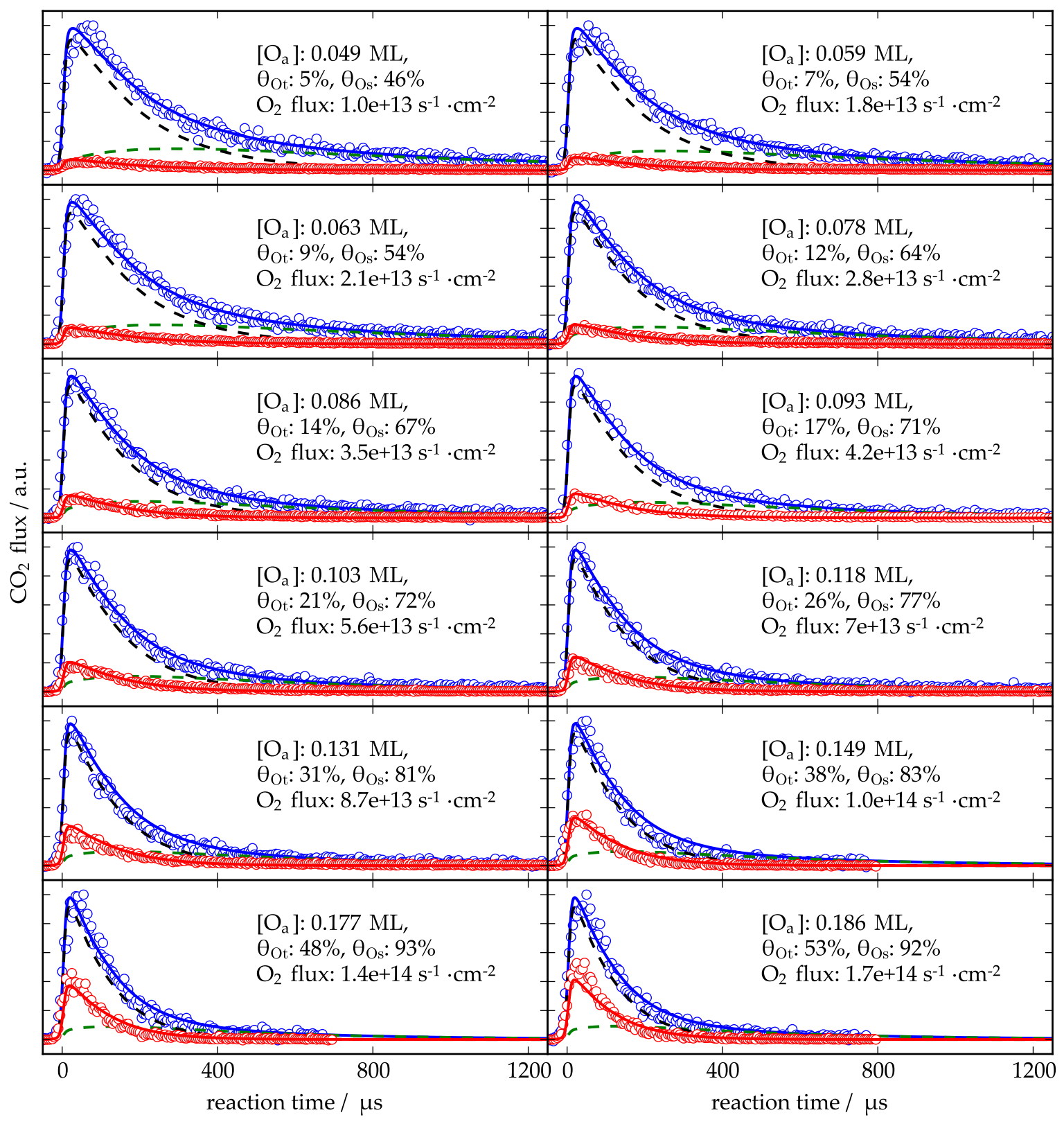

(b) $300^{\circ} \mathrm{C}$, RRRs: $1,1.67,2,2.66,3.33,4,5.29,8.33,10,13.3,16.7$

Figure A.2: Kinetic traces on $\operatorname{Pt}(332)$ (continued). 


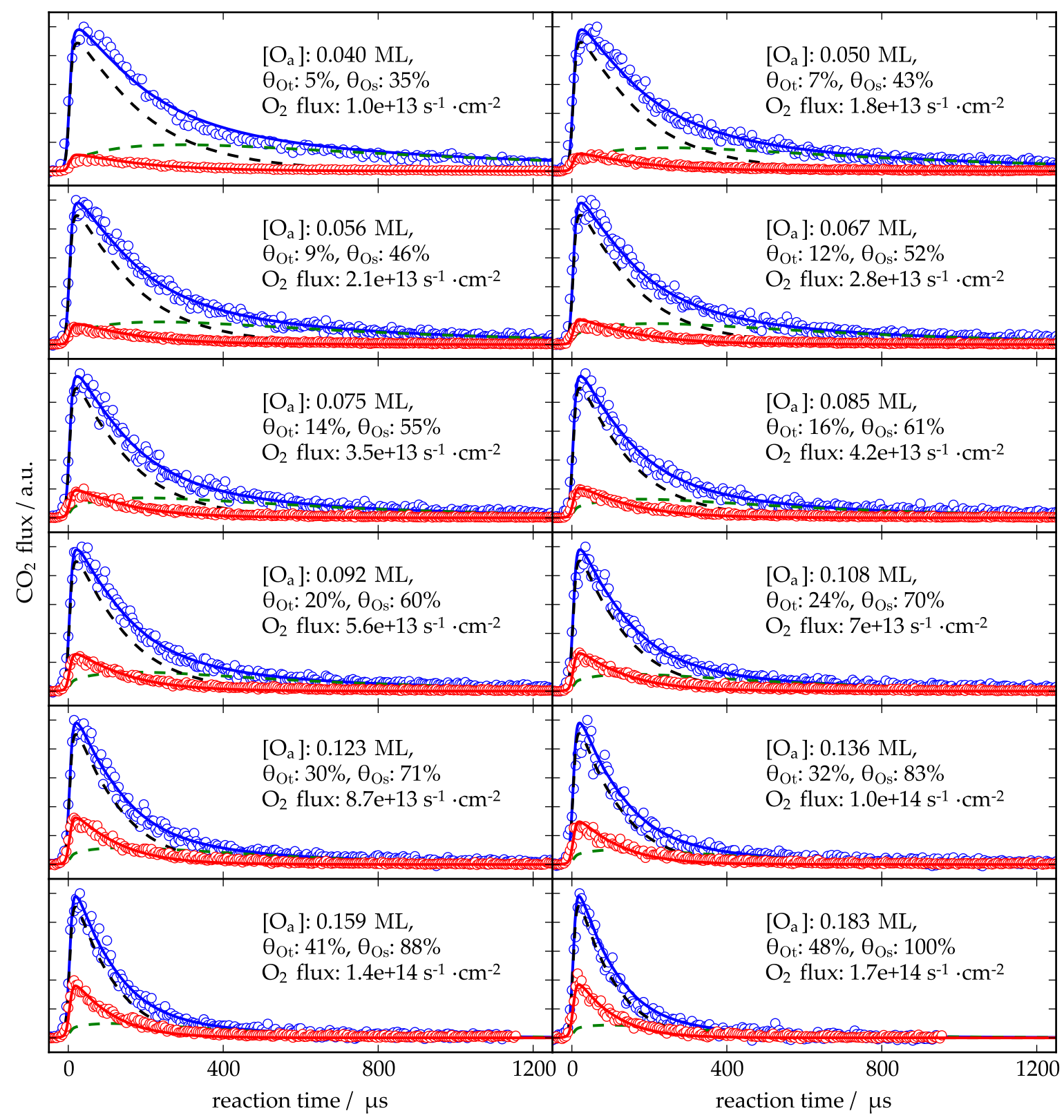

(c) $310{ }^{\circ} \mathrm{C}$, RRRs: $1,1.67,2,2.66,3.33,4,5.29,8.33,10,13.3,16.7$

Figure A.2: Kinetic traces on $\operatorname{Pt}(332)$ (continued). 


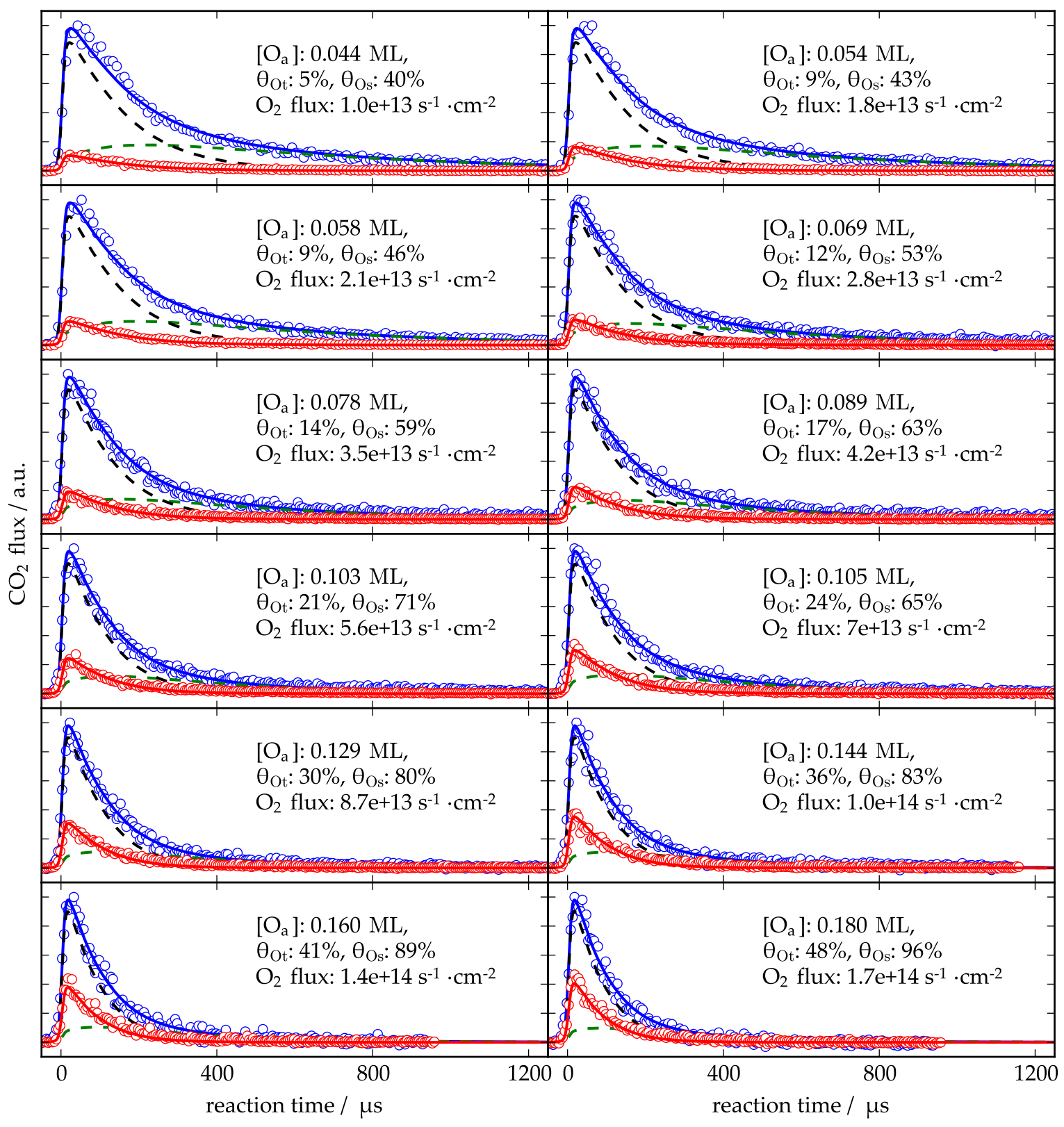

(d) $320^{\circ} \mathrm{C}$, RRRs: $1,1.67,2,2.66,3.33,4,5.29,8.33,10,13.3,16.7$

Figure A.2: Kinetic traces on $\operatorname{Pt}(332)$ (continued). 


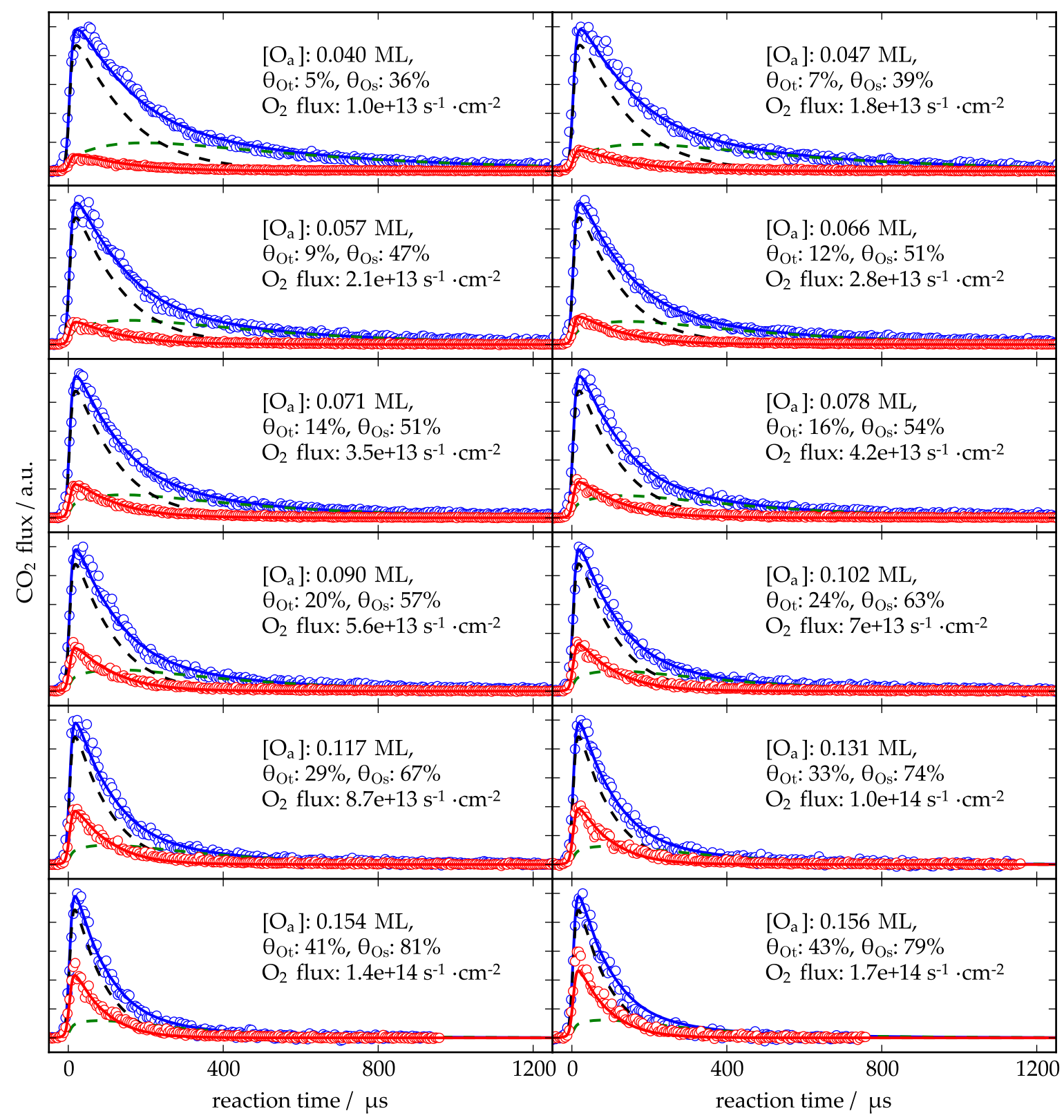

(e) $330^{\circ} \mathrm{C}$, RRRs: $1,1.67,2,2.66,3.33,4,5.29,8.33,10,13.3,16.7$

Figure A.2: Kinetic traces on $\operatorname{Pt}(332)$ (continued). 


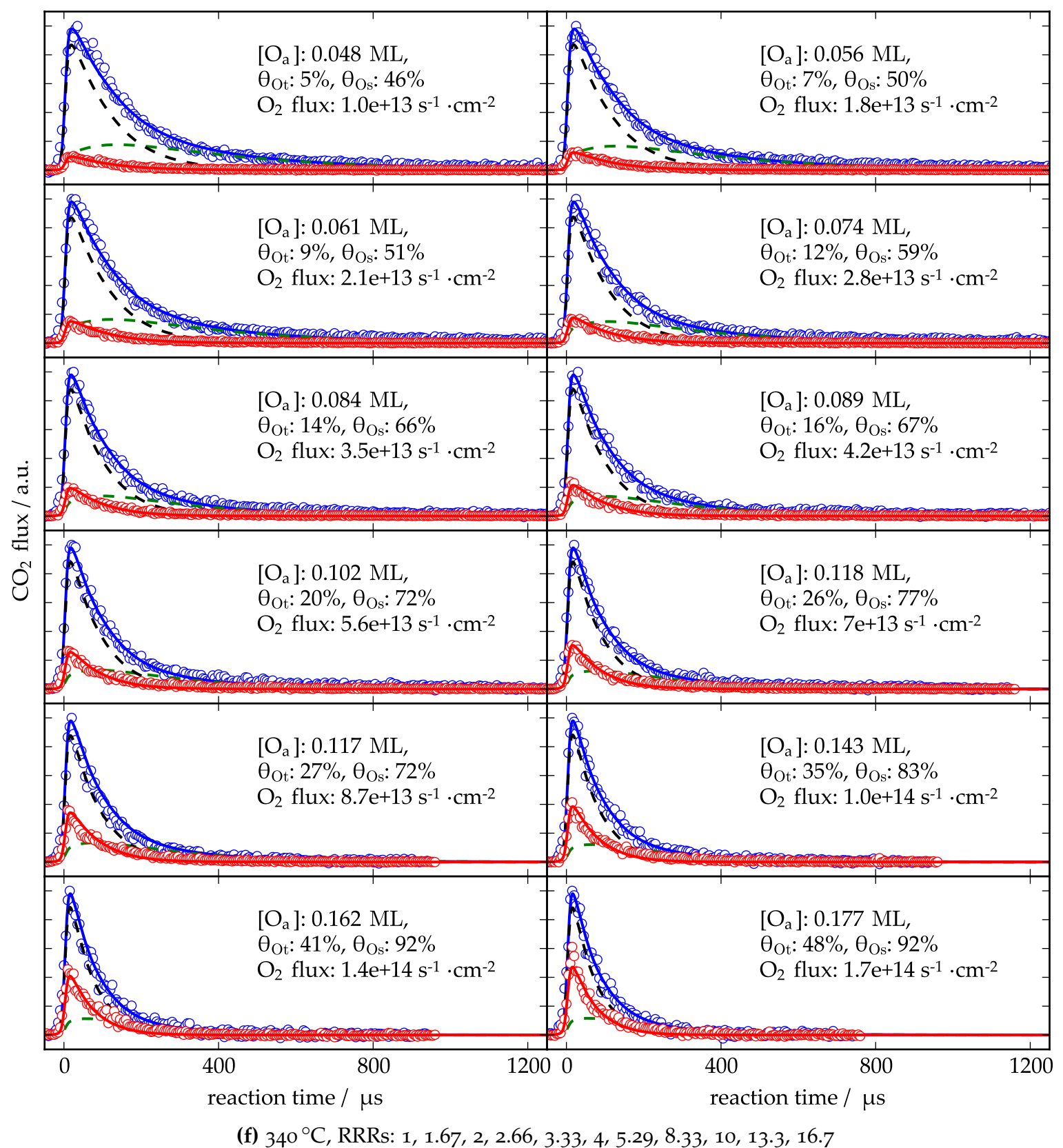

Figure A.2: Kinetic traces on $\operatorname{Pt}(332)$ (continued). 


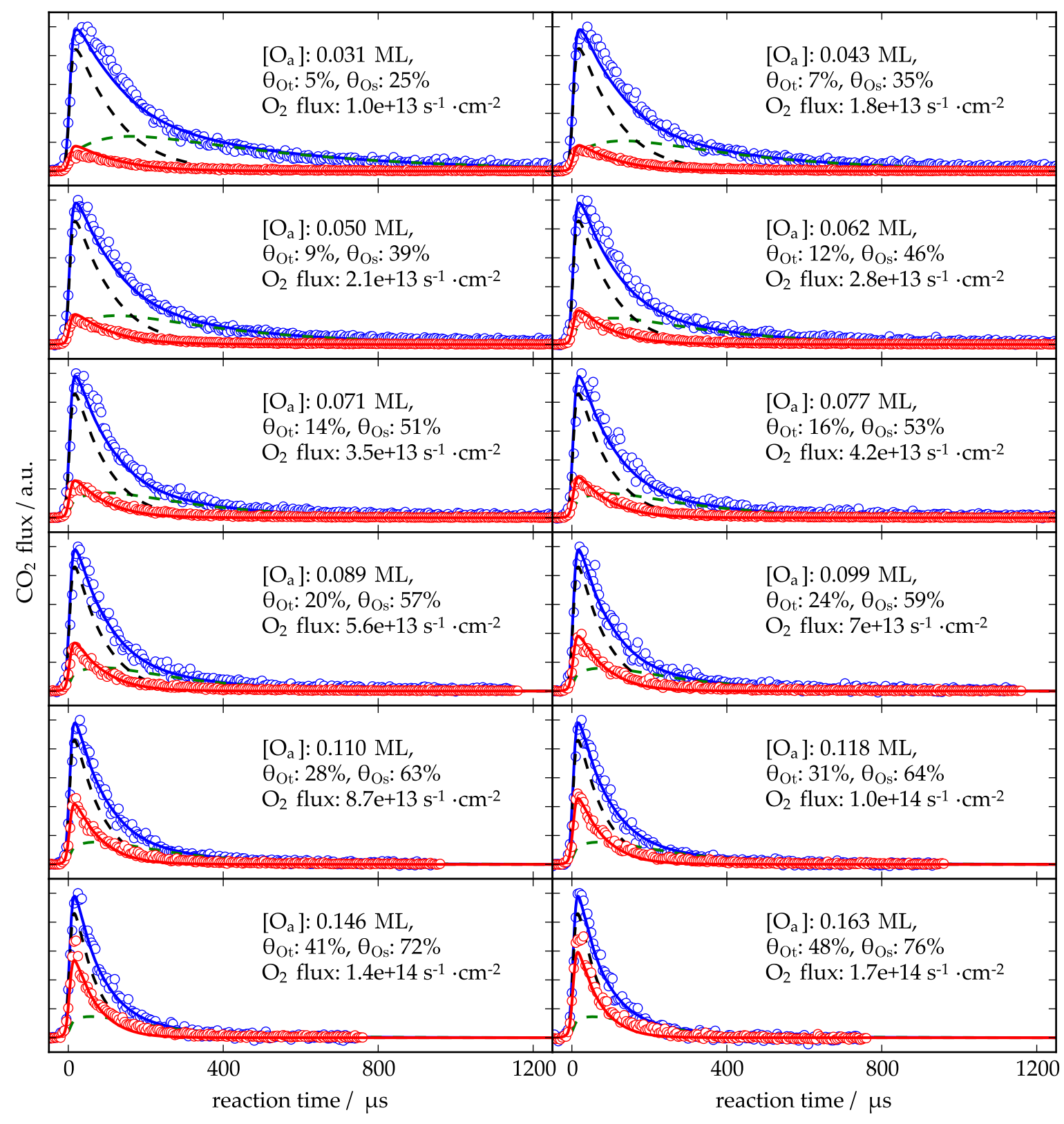

(g) $350^{\circ} \mathrm{C}$, RRRs: $1,1.67,2,2.66,3.33,4,5.29,8.33,10,13.3,16.7$

Figure A.2: Kinetic traces on $\operatorname{Pt}(332)$ (continued). 



\section{B.I SPEED DISTRIBUTIONS IN THE BEAMER II ANALYSIS CODE}

In the python program used to analyze the imaging data at Beamer II several options are available:

- segment (sum)

- line intensity (average)

- $3_{3} \mathrm{D}$ intensity $(\mathrm{r} \sin (\vartheta))$

In addition to these options the bin size $d v$ in $\mathrm{ms}^{-1}$ has to be set. In the first option (segment) the intensity of all pixels in the speed interval $v+\mathrm{d} v$ is is added. Depending on the bin size this can lead to anomalies in the distribution - if the bin size is too small the number of pixels in one speed interval $v+\mathrm{d} v$ might be very different than in the adjacent speed interval. Different patterns can be seen when varying the bin size. At larger bin sizes these effects become less pronounced. If binning can be achieved without anomalies, the resulting signal represents the speed distribution with proper weighing as shown in figure 4.11b.

In the second option (line intensity) this anomaly is prevented by calculating the average intensity of all pixels in a speed interval $v+\mathrm{d} v$. Note that this biases the speed distribution: Ions with higher speeds spread over the same solid angle d $\vartheta$ but occupy a larger area. The ion density per area is thus decreased at high velocities. The total ion count per speed interval is not changed, as the number of pixels per speed interval also increases with speed. However, as the line intensity option calculates the average intensity per speed interval, this leads to a biased speed distribution.

An example can be seen in the inset on the side. The correctly determined speed distribution (segment (sum)) is plotted as a black line, the biased speed distribution obtained by averaging is plotted as a red line. This option was initially introduced to circumvent the binning anomalies.

While writing this, I realized that another option "segment (weighed average)" would be useful and implemented it for future use. This option produces the correct distribution, as in the first option, without

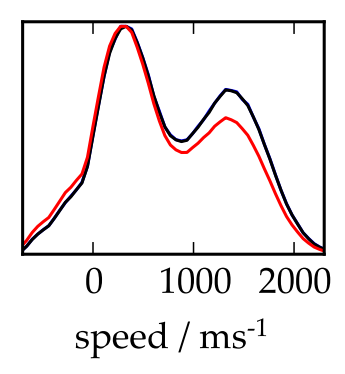
any binning issues.

The last option ( $3 \mathrm{D}$ intensity) weighs each point by $r \cdot \sin (\vartheta)$. This is useful when one wants to compare the total ${ }_{3} \mathrm{D}$ flux of two components. This requires an ion image which samples the distribution 
along $\vartheta$ over the whole range where products appear. Since our ion optics have a maximum acceptance range spanning $70^{\circ}$, this is not possible for a cosine distribution. For now, the option has been disabled to prevent mistakes.

\section{B.2 HOW TO FIT ARRHENIUS PARAMETERS SEPARATELY}

Instead of rate constants k, one typically reports the Arrhenius parameters that characterize the rate constant as

$$
k=A \times \exp \left(-\frac{E_{a}}{k_{B} T}\right),
$$

with the pre-exponential factor A (e.g. in $s^{-1}$ ) and the activation energy $E_{a}$ (in $\mathrm{eV}$ or $\mathrm{kJ} / \mathrm{mol}$ ).

A problem I encountered when fitting the Arrhenius parameters in the kinetic model, was the strong correlation between activation energy and prefactor. This turned out to be very difficult for the optimization routine to handle. Before presenting the solution I came up with, I describe the reason for this difficulty using figure B.1.

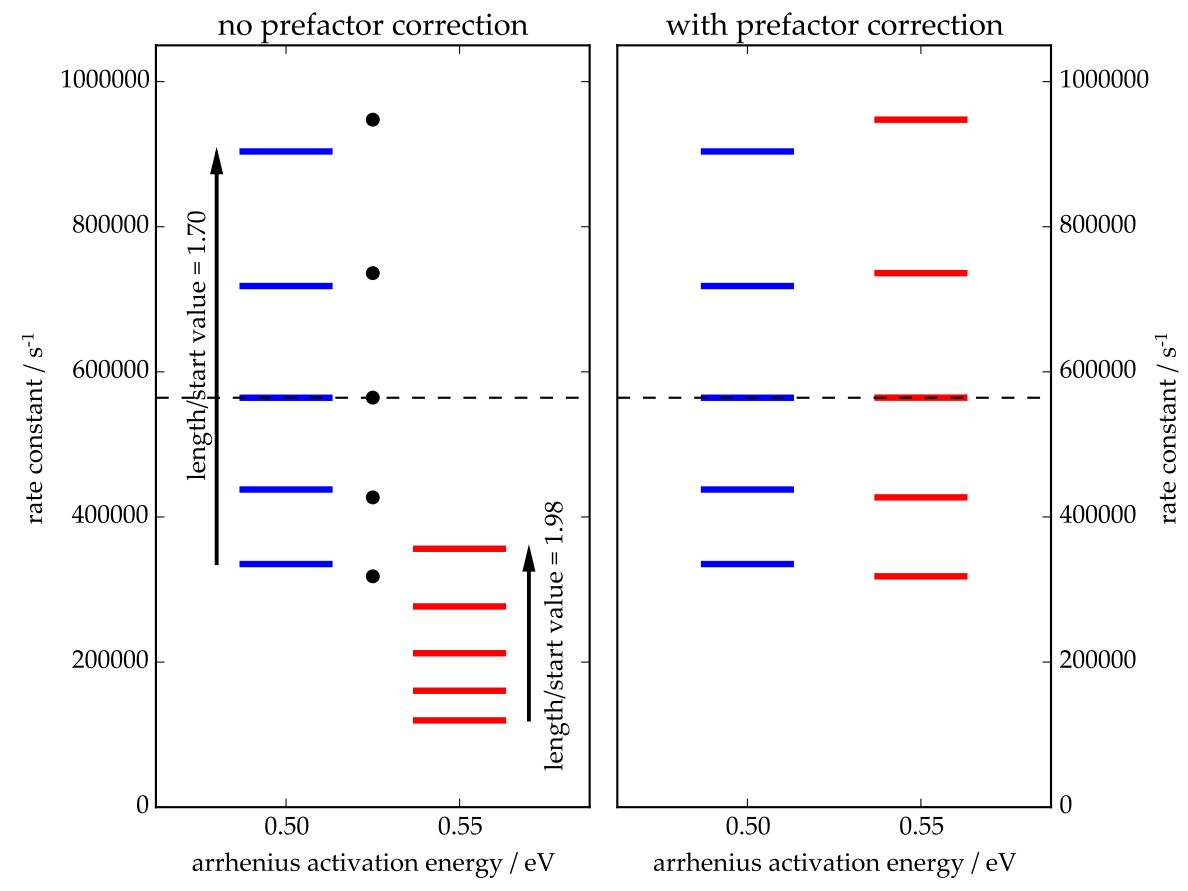

Figure B.1: Arrhenius parameter interaction: The effect of the activation energy on the rate constant is illustrated in the left panel. The red and blue lines depict rate constants at five different temperatures for two different energies. The right panel shows a result, where a compensating prefactor has been added to the equation, see text.

Consider that you want to determine a single rate coefficient in a kinetic model by fitting the respective Arrhenius parameters. Assume 
that a linear regression of $\ln (k)$ vs $1 / T$ is not possible. You have experimentally determined the rate constant at 5 different temperatures $(563,578,593,608$ and $623 \mathrm{~K})$. This is plotted as black dots on the left side of figure B.1.

You pass an initial guess of $0.5 \mathrm{eV}$ for the activation energy and an arbitrary pre-exponential factor of $1 \times 10^{10} \mathrm{~s}^{-1}$ to the optimization method. The values for the rate constant the optimization method calculates at the five different temperatures is plotted as blue lines. When comparing to the experimental data points (black dots) you see that the value for the rate constant is too large at the lowest temperature and too small at the highest temperature - the change with temperature is too small.

The relative change with temperature, which can be expressed as the length of the arrow in figure B.I (absolute change with temperature) relative to the value where it starts, is greater for a higher activation energy. The optimization method tries to increase the activation energy, which is a step in the right direction. Because the activation energy also influences the absolute magnitude of the rate constant, the absolute values of the rate constant at the five different temperatures decrease strongly (red lines). This leads to a large mismatch and from the residual alone the solver will assume that this was a step in the wrong direction. The absolute magnitude of the rate constant can easily be increased by using a higher prefactor, but the solver does not know this.

As a result of the poor agreement the step is rejected. The only way for the solver to reach the global minimum is to vary the activation energy and prefactor alternately and in very small steps. The calculations presented in this thesis were quite time-consuming. The solver thus needed to converge more efficiently than described above.

One way to decouple the two is to introduce a compensating prefactor $A_{\text {comp }}$ to the equation,

$$
A_{\text {comp }}=\exp \left(-\frac{\left(-E_{a}^{o l d}+E_{a}^{n e w}\right)}{k_{B} T_{\text {fixed }}}\right),
$$

where $E_{a}^{o l d}$ is the old activation energy, $E_{a}^{n e w}$ is the new value and $\mathrm{T}_{\text {fixed }}$ is the middle temperature as depicted by the black dashed line on the right in figure B.I. The rate constant is then calculated as

$$
k=A \times A_{\text {comp }} \times \exp \left(-\frac{E_{a}}{k_{B} T}\right) .
$$

The result of changing the activation energy from $0.5 \mathrm{eV}$ to $0.55 \mathrm{eV}$ in Eq. B. 3 is plotted as red lines. When using this equation, the rate constant at the middle temperature $\left(\mathrm{T}_{\text {fixed }}\right)$ is not varied upon changing the activation energy. The activation energy now only scales the relative change with temperature. The prefactor $A$ controls the absolute magnitude. 
As the solver can independently vary the relative change and the absolute magnitude, it will converge much faster.

\section{B.3 PYTHON CLASS STRUCTURE OF THE KINETIC MODEL}

An overview of the different classes used in the fit routine is shown in figure B.2.

The code is divided into three classes, which will be introduced in the order they are called. The class C02_model_fitter on the left is initialized first and imports experimental data. The function global fit_objective, which takes a set of input parameters (rate coefficients) and calculates and returns the global residual, is passed to an optimization routine (lmfit.minimize, not shown here), which alters the parameters to minimize the global residual. Per function call of global_fit_objective one instance of local_fit is called per kinetic trace. The local_fit calls are parallel (multi-threading), which is indicated by the double arrows and the star.

The local_fit function imports previously saved $\mathrm{O}_{t}$ and $\mathrm{O}_{s}$ values for this kinetic trace, runs the kinetic_model_wrapper and compares the numeric solution to the kinetic trace. A local optimization, which alters $\mathrm{O}_{t}$ and $\mathrm{O}_{s}$ within defined boundaries, then minimizes this local residual. The optimum values for $\mathrm{O}_{t}$ and $\mathrm{O}_{s}$ are stored for the next call and the resulting local residual is returned to the global_fit_objective.

The class kinetic_model_wrapper is initiated with several input parameters: temperature, Arrhenius parameters, initial concentrations and incoming beam profile. The Arrhenius parameters (activation energy and prefactor) are used to calculate the rate constants at the input temperature. A time-array of interest is generated and an instance of the class step_terrace_model is created. Executing the function step_terrace_model. solve returns the concentrations at the times defined in the time-array of interest. The kinetic_model_wrapper uses these concentrations to calculate the relevant flux, and returns the flux vs time arrays to the local_fit. Optionally, the function kinetic_model_wrapper.plot_solution can be called to plot the result, as shown in figure 7.11. 

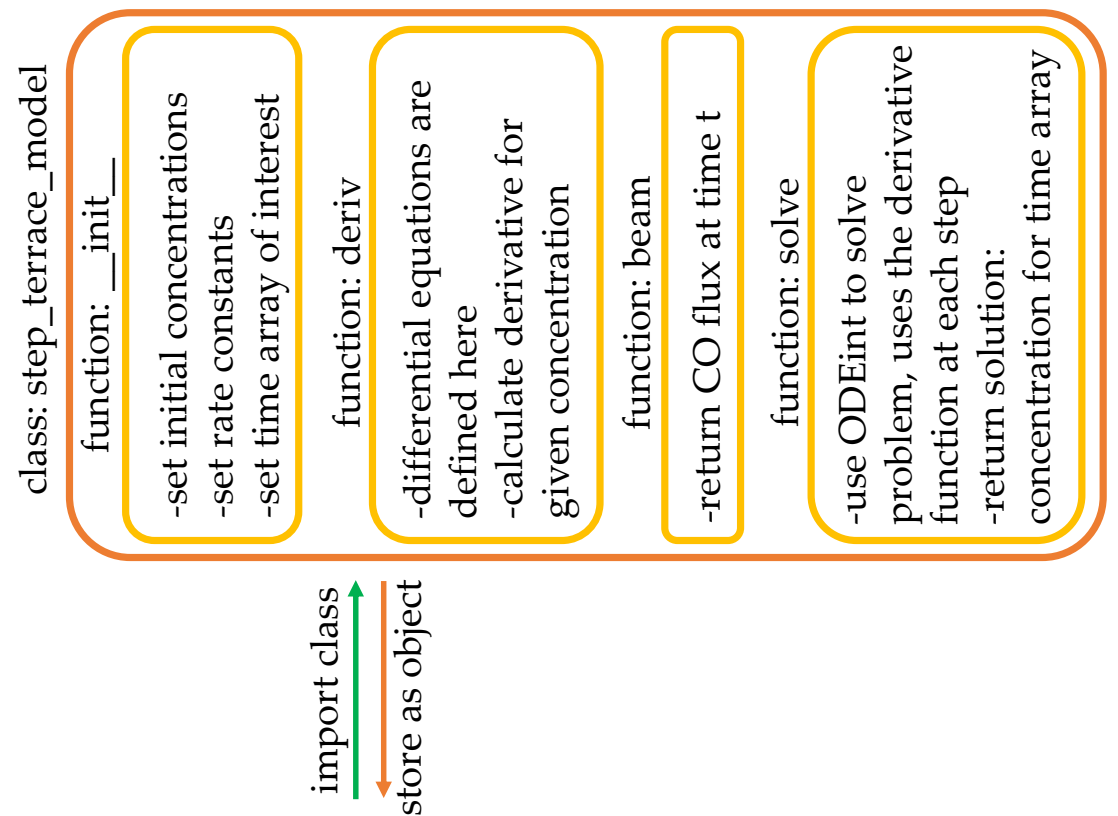

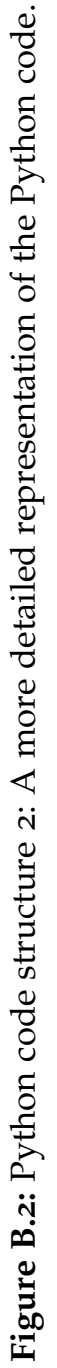

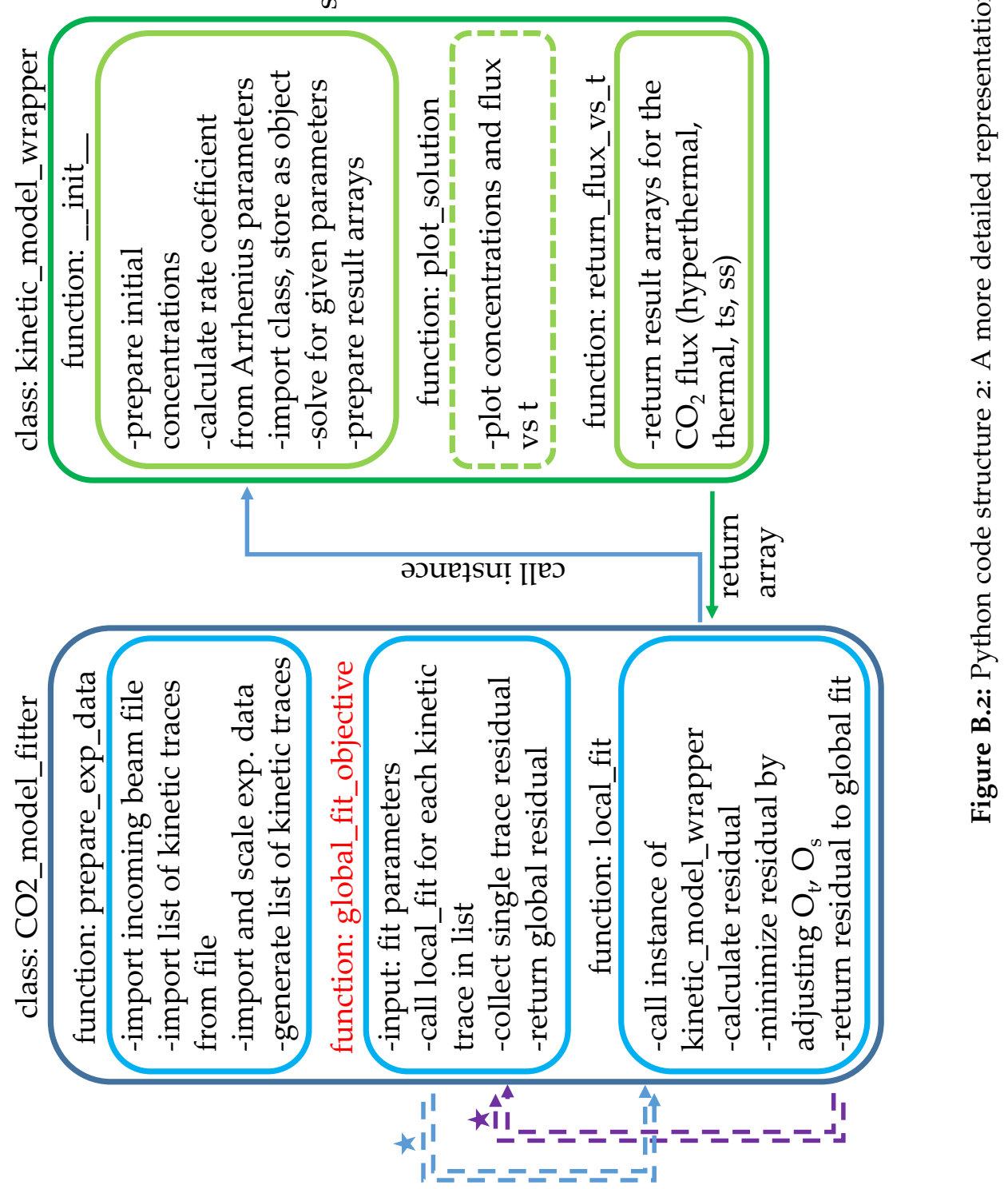



EXTENDED EXPERIMENTAL TECHNIQUES AND TIPS

\section{C.I CONVERSION OF PIXELS TO MM}

The calibration of the screen size is straightforward. Record the size of the MCPs using e.g. background noise. The best results are obtained when the MCP and phosphor screen are not be gated. An example image is shown in figure C.I.

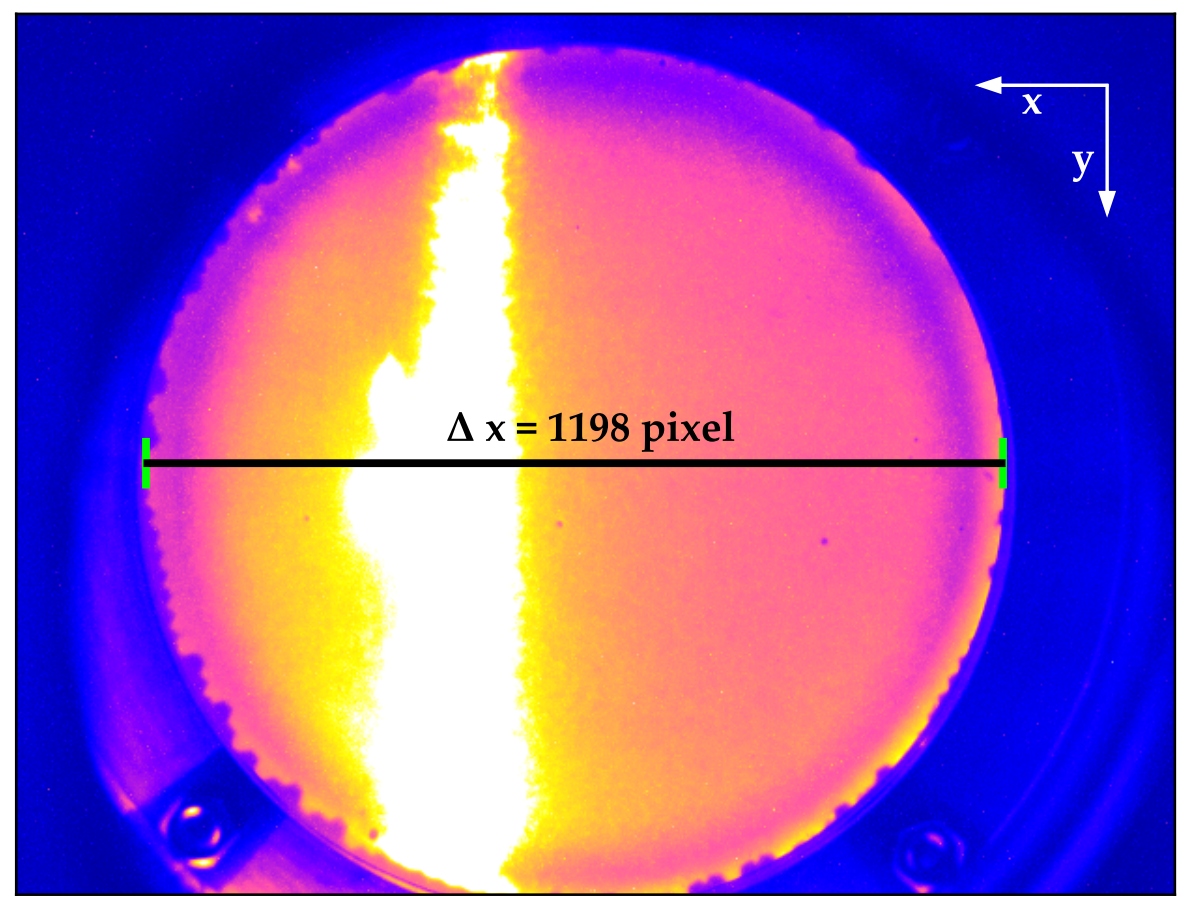

Figure C.1: Screen size calibration image.

The ion signal in the middle of the screen can simply be ignored. The important information seen in this image is the size of the MCP. The edge of the MCP in magenta stands out clearly against the blue background. At the jagged outer diameter one can still see the hexagonal shape of the individual channels of the MCP. The size of the MCP in pixels can be measured directly from the ion image, as annotated in the image. As the MCP has a diameter of $52 \mathrm{~mm}$ we can calculate the calibration factor as

$$
x=\frac{1198 \mathrm{pixel}}{52 \mathrm{~mm}}=23.0 \mathrm{pixel} / \mathrm{mm} \text {. }
$$


A re-calibration of the pixel-to-mm conversion is necessary if the distance of the camera from the window flange and thus the distance from the phosphor screen is changed.

\section{C.2 MULTIPLE BEAM PULSE TIMING}

In the $\mathrm{CO}$ oxidation experiments two molecular beams were used to dose the surface with $\mathrm{O}_{2}$ and $\mathrm{CO}$. As we wanted to control the repetition rates separately, the molecular beams were triggered by different delay generators. The operational principle is explained using figure C.2.

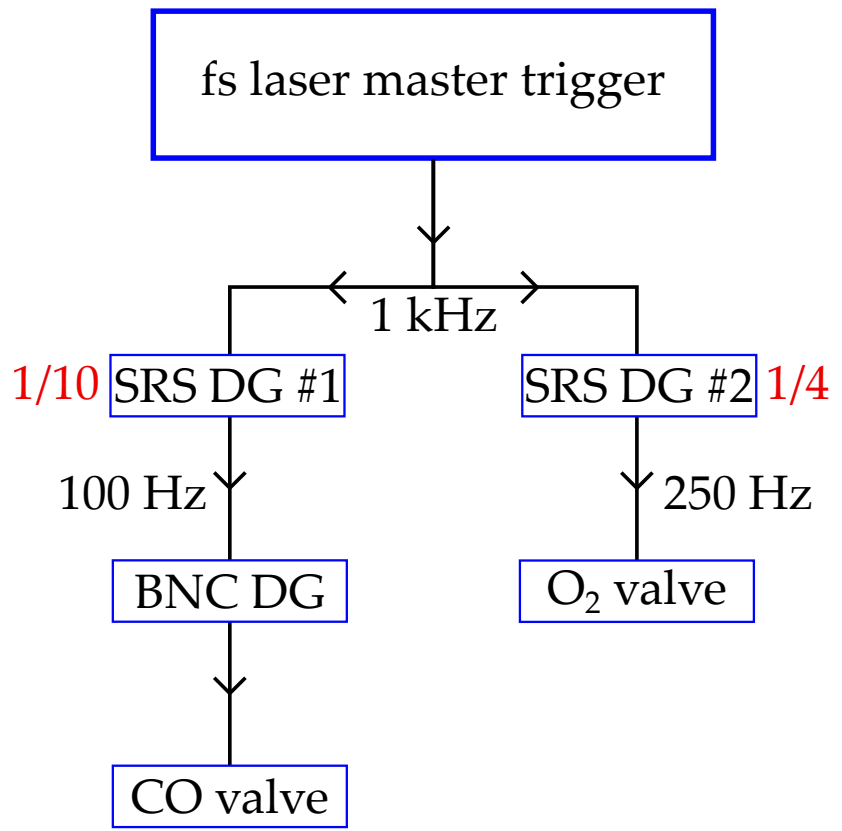

Figure C.2: Control schematic of the two molecular beam valves.

The fs laser Q-switch is used as master and triggers two SRS DG 535 delay generators. By setting an otherwise unused channel on these delay generators to output a long pulse, the delay generator can be used as a pulse picker (frequency divider). If the input frequency is $1 \mathrm{kHz}$ and the $\mathrm{C} \sqcap \mathrm{D}$ gate on the delay generator has a width of $1.9 \mathrm{~ms}$, it will be busy during the next input trigger pulse and thus miss it. The SRS DG \#2, which controls the $\mathrm{O}_{2}$ beam using the $A \sqcap D$ gate, will then be triggered every second input trigger pulse. A pulse width of $1.9 \mathrm{~ms}$ thus leads to a period of $2 \mathrm{~ms}$ and the output frequency is $500 \mathrm{~Hz}$. A pulse width of $3.9 \mathrm{~ms}$ (a period of $4 \mathrm{~ms}$ ) results in an output frequency is $250 \mathrm{~Hz}$ etc. Picking every 1oth pulse would lead to a repetition rate of $100 \mathrm{~Hz}$, as indicated on the left in figure C.2.

As a consequence of this setup the two SRS delay generators are independent of each other. If both are forwarding every second pulse they might be running on the same master trigger pulse or they might 
be alternating $1 \mathrm{~ms}$ out of phase. If the repetition rates of the two delay generators are not a multiple of each other, the beam-beam delay will change as they cycle through different master trigger pulses. In order to prevent the $\mathrm{O}_{2}$ beam from accidentally passing through the ionizing laser pulse it was always triggered $100 \mu$ s after the Q-switch. This ensures that the $\mathrm{O}_{2}$ pulse has at least $900 \mu$ s to fly towards the surface and clear the ionization volume.

Using non-synchronized molecular beam pulses leads to another possible complication: If the beam-laser delay between $\mathrm{CO}$ pulse and fs-laser is greater than $1000 \mu$ s the $\mathrm{O}_{2}$ pulse might arrive while the $\mathrm{CO}$ on the surface is still reacting. As the dose per $\mathrm{O}_{2}$ pulse is small compared to the amount of O-atoms already on the surface, this was not considered a problem. 
Jannis Neugebohren

Hennebergstr. 12

37077 Göttingen
Day of birth: January 28th, 1989

Place of birth: Uelzen

Nationality: German

EDUCATION

Since $06 / 2013$

Georg-August-University of Göttingen

PhD student in Physical Chemistry

$09 / 2012-03 / 2013$

Dalian Institute of Chemical Physics

Master thesis abroad in PR China

$11 / 2011-04 / 2013$

Georg-August-University of Göttingen

Master of Science in Chemistry

$10 / 2007-09 / 2011$

Georg-August-University of Göttingen

Bachelor of Science in Chemistry

08/2001 - 06/2007 Lessing-Gymnasium Uelzen

Abitur

List of Publications

[1] Dan J. Harding, Jannis Neugebohren, M. Grütter, A. F. SchmidtMay, D. J. Auerbach, T. N. Kitsopoulos, and A. M. Wodtke. "Single-field slice-imaging with a movable repeller: Photodissociation of $\mathrm{N}_{2} \mathrm{O}$ from a hot nozzle." In: The Journal of Chemical Physics 141.5 (Aug. 2014), p. 054201. DoI: 10.1063/1. 4891469.

[2] Dan J. Harding, Jannis Neugebohren, Daniel J. Auerbach, T. N. Kitsopoulos, and Alec M. Wodtke. "Using Ion Imaging to Measure Velocity Distributions in Surface Scattering Experiments." In: Journal of Physical Chemistry A 119.50 (2015), pp. 1225512262. DOI: 10.1021 /acs . jpca. 5 b06272.

[3] Alice F. Schmidt-May, Monika Grütter, Jannis Neugebohren, T. N. Kitsopoulos, Alec M. Wodtke, and Dan J. Harding. "Rotationally Resolved Vacuum Ultraviolet Resonance-Enhanced Multiphoton Ionization (VUV REMPI) of Acetylene via the G Rydberg State." In: The Journal of Physical Chemistry A 120.27 (July 2016), pp. 5399-5407. DOI: 10.1021/acs . jpca. 6b02477. 
[4] Dan J. Harding, Jannis Neugebohren, Hinrich Hahn, D. J. Auerbach, T. N. Kitsopoulos, and Alec M. Wodtke. "Ion and velocity map imaging for surface dynamics and kinetics." In: The Journal of Chemical Physics 147.1 (2017), p. 013939. DOI: 10. 1063/1. 4983307.

[5] Jannis Neugebohren, Dmitriy Borodin, Hinrich W. Hahn, Jan Altschäffel, Alexander Kandratsenka, Daniel J. Auerbach, Charles T. Campbell, Dirk Schwarzer, Dan J. Harding, Alec M. Wodtke, and Theofanis N. Kitsopoulos. "Velocity-resolved kinetics of site-specific carbon monoxide oxidation on platinum surfaces." In: Nature 558.7709 (June 2018), pp. 280-283. Dor: 10 . 1038 / s41586-018-0188- $\mathrm{x}$.

29.06.2018, Jannis Neugebohren 